\title{
Allene, Dihydronaphthalenon-Derivate und andere Sekundärmetabolite aus Pilzen mariner Habitate sowie Beiträge zu deren Biosynthese
}

\author{
DISSERTATION \\ zur Erlangung des Doktorgrades \\ der Mathematisch-Naturwissenschaftlichen Fakultäten \\ der Georg-August-Universität zu Göttingen
}

\author{
vorgelegt von \\ Diana WolfF
}

aus Schwedt/Oder

Göttingen 2004 
D 7

Referent: Prof. Dr. A. ZEECK

Korreferent: Prof. Dr. U. DiederICHSEN

Tag der mündlichen Prüfung: 03.11.2004 
Die vorliegende Arbeit wurde im Institut für Organische und Biomolekulare Chemie der Universität Göttingen von Januar 2001 bis September 2004 unter Anleitung von Prof. Dr. A. ZeECK durchgeführt.

Herrn Prof. Dr. A. ZeECK danke ich für die spannende Aufgabenstellung, seine stete Förderung, die wertvollen Diskussionen und Anregungen sowie sein Interesse am Fortgang dieser Arbeit. 


\section{A. Theoretischer Teil}

1. Einleitung 1

1.1.Pilze als Arzneimittelquelle 1

1.2. „Marine“ Pilze und ihre Sekundärmetaboliten 4

2. Aufgabenstellung 11

3. Chemisches Screening 12

3.1.Screening 12

3.2. Durchführung des Chemischen Screenings 14

$\begin{array}{ll}\text { 3.3. Stammauswahl } & 15\end{array}$

4. Stämme mit literaturbekannten Metaboliten 17

$\begin{array}{ll}\text { 4.1.WDM35 } & 17\end{array}$

4.1.1. Isolierung und Charakterisierung der Metabolite 17

4.2.WDMHC2 26

4.2.1. Isolierung und Charakterisierung von Cyclonerodiol 26

4.3. WDMH24 28

4.3.1. Isolierung und Charakterisierung von $\mathrm{N}$-(2-cis(4-Hydroxy$\begin{array}{ll}\text { phenyl)ethenyl)-formamid } & 28\end{array}$

4.4.WDMH51 30

4.4.1. Isolierung und Charakterisierung der Metabolite 30

5. Stamm WDMH28 39

5.1. Fermentation und Isolierung der Metabolite 39

5.2. Charakterisierung der Metabolite 40

5.3. Biogeneseuntersuchungen 49

5.3.1. Biosynthese-Experimente aus der Literatur 49

5.3.2. Eigene Biogeneseuntersuchungen 53

5.3.2.1. Fermentationskurve 53

5.3.2.2. Fütterungsexperimente am 6-Methoxyparvulenon 54

5.3.2.3. Fütterungsexperimente am Naphthalacton A 58

6. Stamm WDMH46 65

6.1. Allene $\quad 65$ 
6.2. Fermentation und Isolierung der gebildeten Metabolite 68

6.3. Charakterisierung der Lupallene 69

6.4. Variation der Kultivierungsbedingungen 76

6.5. Isolierung und Charakterisierung der neuen Metabolite 82

6.6. Derivatisierung der Allene 94

6.6.1. Acetylierung von Lupallen C 95

6.6.2. Umsetzung von Lupallen C mit ortho-Brombenzoesäure 96

6.6.3. Umsetzung von Lupallen A mit para-Brombenzoesäure 97

$\begin{array}{ll}\text { 6.7. Biogeneseuntersuchungen } & 98\end{array}$

6.7.1. Bekanntes zur Biosynthese von Allenen 98

6.7.2. Zur Biogenese der Allene aus WDMH46 105

$\begin{array}{ll}\text { 6.7.2.1. Fermentationskurve } & 105\end{array}$

6.7.2.2. Fütterungsexperimente mit $\left[1-{ }^{13} \mathrm{C}\right]$-Acetat 106

6.7.2.3. Mevalonat- vs. Nicht-Mevalonat-Weg 108

6.7.2.4. Fütterungsexperimente mit $\left[\mathrm{U}^{13} \mathrm{C}_{3}\right]$-Glycerin 110

6.7.2.5. Zur Biosynthese von Alkannin und Shikonin 113

6.7.2.6. Zufütterung potentieller Biosynthesevorläufer 116

6.7.2.7. Zur Biosynthese der WDMH46-Metabolite 117

6.8. Zur biologischen Aktivität der Lupallene 119

$\begin{array}{ll}\text { 7. Zusammenfassung der Ergebnisse } & 121\end{array}$

\section{B. Experimenteller Teil}

$\begin{array}{ll}\text { I. Allgemeines } & 125\end{array}$

1. Instrumentelle Analytik 125

2. Chromatographische Methoden 127

3. Mikrobiologische Methoden 130

II. Arbeiten an marinen Pilzstämmen 132

1. Stammhaltung 132

1.1. Agarplatten 132

1.2. Stickstoff-Einlagerungen 132

1.3. Glycerin-Einlagerungen 133 
3. Stamm WDMH35

3.1. Kultivierung und Aufarbeitung

3.2. Isolierung

3.3. Charakterisierung der Metabolite

4. Stamm WDMHC2

4.1. Kultivierung und Aufarbeitung

4.2. Cyclonerodiol

5. Stamm WDMH24

5.1. Kultivierung und Aufarbeitung

5.2. N-(2-cis-(4-Hydroxyphenyl)ethenyl)-formamid

6. Stamm WDMH51

6.1. Kultivierung und Aufarbeitung

6.2. Isolierung

6.3. Charakterisierung der Metabolite

7. Stamm WDMH28

7.1. Kultivierung und Aufarbeitung

7.2. Isolierung

7.3. Charakterisierung der Metabolite

7.4. Acetylierung von 6-Methoxyparvulenon

7.5. Wachstumskurve

7.6. Biogeneseuntersuchungen

8. Stamm WDMH46

8.1. Kultivierung 156

8.2. Variation der Kultivierungsbedingungen 156

8.2.1. Kultivierung in Schüttelkolben mit XAD-Zusatz 156

8.2.2. Kultivierung in Schüttelkolben mit Salz-Zusatz 156

8.2.3. Kultivierung in Schüttelkolben als Ruhekultur mit und ohne Salz 
8.2.5. Kultivierung in P-Kolben als Festkultur

8.2.6. Kultivierung in Fermentern

8.3. Aufarbeitung

8.4. Isolierung und Charakterisierung der Metabolite

8.5. Wachstumskurve

8.6. Biogeneseuntersuchungen

8.6.1. Fütterung von $\left[1-{ }^{13} \mathrm{C}\right]$-Acetat und $\left[\mathrm{U}-{ }^{13} \mathrm{C}_{3}\right]$-Glycerin

8.6.2. Fütterung potentieller Vorläufer

8.7. Derivatisierungen

8.7.1. Acetylierung von Lupallen $\mathrm{C}$

8.7.2. ortho-Brombenzoat von Lupallen $\mathrm{C}$ 178

8.7.2. para-Brombenzoat von Lupallen $A$ 


\section{A. THEORETISCHER TEIL}

\section{Einleitung}

\subsection{Pilze als Arzneimittelquelle}

Während Pflanzen schon seit jeher zur Heilung von Krankheiten und zur Linderung leichter Beschwerden benutzt wurden, ${ }^{1}$ waren Pilze meist als Krankheitserreger bekannt. Eine im Mittelalter weit verbreitete Plage war das St. Anthony's Fire, auch Ergotismus genannt. Im Verlauf von Epidemien litten ganze Dörfer an Krämpfen, Halluzinationen und an Gewebsnekrosen, die zu einer tödlich verlaufenden Fäulnis der Glieder führte. Im Jahr 922 sollen dieser Krankheit in Spanien und Frankreich rund 40000 Menschen erlegen sein. ${ }^{2}$ Erst im 17. Jahrhundert wurde die Ursache des St. Antoniusfeuers bekannt. Die Vergiftungserscheinungen sind auf den Verzehr von Mutterkorn verseuchtem Roggenmehl oder Brot zurückzuführen. Der Schimmelpilz nistet sich in Getreideähren, vor allem zwischen Roggenkörnern ein und verwandelt das Korn in blauschwarze Gebilde. 1853 wurde der Pilz Claviceps putpurea als eigentlicher Schadenserreger entdeckt. $^{2}$ Heute kennt man auch die giftigen Stoffe, die diese Krankheit verursachen. Eine dieser Substanzen ist das Ergotamin (1).

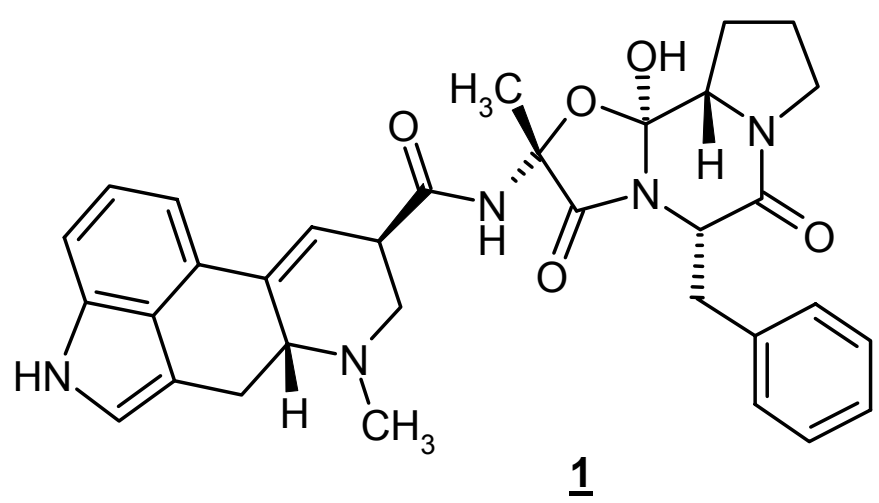

Diese Substanz zeigt hohe Affinitäten zu $\alpha_{1}$ - und $\alpha_{2}$-Adrenozeptoren, zu Dopaminsowie Serotonin-(5-HT)-Rezeptoren. In hohen Dosen bewirkt es, dass Blutgefäße sich krampfartig verengen, Arme und Beine werden nur noch schlecht durchblutet und schmerzen. Aber wie sagte einst schon Paracelsius: „Alle ding sind gift und 
nichts on gift; alein die dosis macht das ein ding kein gift ist". ${ }^{3}$ In niedriger Dosierung ist das Ergotamin sogar heilsam. Neben seiner Migränewirksamkeit wurde lange Zeit die wehenfördernde, uteruskontrahierende Wirkung des Ergotalkaloids ausgenutzt (Herkunft des Namen Mutterkorn).

Trotz der interessanten medizinischen Eigenschaften der Ergotalkaloide sind Pilze erst durch ihre antibiotischen Eigenschaften ins Interesse der ArzneimittelForschung gerückt. Bereits gegen Ende des 19. Jahrhunderts erkannten Ärzte wie J.B. SANDERSON, W. ROBERTS und TYNDALL antagonistische Effekte von Schimmelpilzen der Gattung Penicillium auf Bakterien. ${ }^{4} 1884$ behandelte J. LISTER den Abszess einer Krankenschwester mit Penicillium glaucum. ${ }^{4}$ Erst 1896 wurde die antibakterielle Wirkung von Penicillium-Kulturen im Tierversuch nachgewiesen. ${ }^{5}$ E. DUCHESNE beimpfte Ratten mit Bacterium coli und Bacterium thyphosis und mischte bei manchen Tieren zu der Bakteriensuspension eine Kultur von Penicillium glaucum bei. Diese Tiere blieben am Leben, während die anderen innerhalb von 24 Stunden verstarben. All diese Erkenntnisse blieben jedoch ohne Resonanz in der wissenschaftlichen Welt. Im Jahr 1896 gelang es Gosıo, erstmals eine Substanz mit antibakteriellen Eigenschaften $\mathrm{zu}$ isolieren. ${ }^{6}$ Sie ist das Gärungsprodukt von Penicillium glaucum und wurde später als Mycophenolsäure (2) identifiziert. ${ }^{7}$

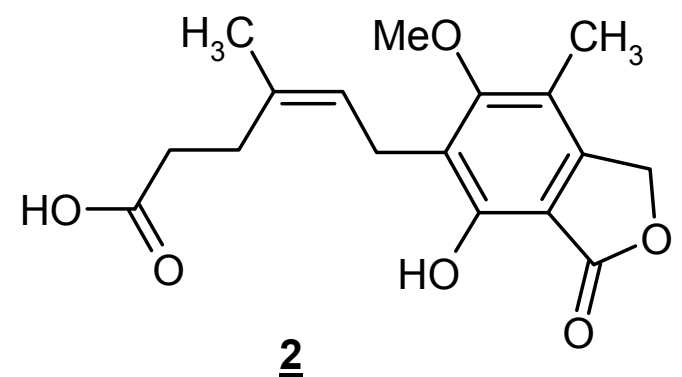

Mycophenolsäure (2) ist ein hochwirksames Immunsuppressivum und wird heute als 2-Morpholinoethylester (CellCept ${ }^{\circledR}$, FA. ROCHE) in der Transplantationsmedizin zur Vorbeugung von Organabstoßungen bei Patienten nach Herz-, Leber- oder Nierentransplantationen angewendet. Die Wirkung beruht auf der selektiven, nicht kompetitiven und reversiblen Hemmung der Inosinmonophosphat-Dehydrogenase (IMPDH), wodurch selektiv die DNA-Synthese von B- und T-Lymphozyten unterdrückt wird. Seit kurzem steht in der Schweiz ein ähnliches Medikament 
(Mycophenolat-Natrium, Myfortic ${ }^{\circledR}$, NovARTIS) mit verbesserten pharmakologischen Eigenschaften zur Verfügung.

Der große Durchbruch in der Arzneimittel-Forschung gelang aber erst mit der Entdeckung des Penicillins (יㅜ) 1928 fand A. FLEMING den bakteriostatischen Wirkstoff als Ursache für die wachstumshemmende Wirkung des Schimmelpilzes Penicillium notatum auf Bakterien und deutete in seinen Veröffentlichungen vorsichtig an, dass sich Penicillin ( $\underline{3}$ ) als Medikament eignen könnte. ${ }^{8}$ Auch FLEMMINGs Arbeiten fanden zunächst kaum Beachtung. Erst die Isolierung und Reinigung größerer Penicillin-Mengen durch H.W. FLOREY und E.B. ChAIN brachten den Durchbruch. ${ }^{9} 1941$ erfolgte die Strukturaufklärung des Wirkstoffes und eine Weiterverarbeitung bis hin zum ersten klinischen Präparat.<smiles>CC(=O)OCC1=C(C(=O)O)N2C(=O)[C@@H](NC(=O)CCCC(N)C(=O)O)[C@H]2SC1</smiles>

Im Jahr 1945 setzte G. BROTZU auf Sardinien einen weiteren Meilenstein auf dem Weg zur Entwicklung potenter Antibiotika. ${ }^{10} \mathrm{Er}$ erforschte Meerwasserproben aus der Nähe eines Abwasserauslaufes und isolierte daraus den Pilz Acremonium chrysogenum. Dessen Inhaltsstoffe wirkten nicht nur gegen gram-positive Bakterien, wie Staphylokokken, sondern auch gegen gram-negative Bakterien, beispielsweise Typhuserreger. In den folgenden Jahren gelang die Identifizierung und Strukturaufklärung der biologisch aktiven Substanz zu Cephalosporin C (4). ${ }^{11}$ Die Cephalosporine weisen, wie die Penicilline, als Grundelement den viergliedrigen $\beta$-Lactamring auf, der für die Wirkung (Blockierung der ZellwandSynthese der Bakterienzelle) verantwortlich gemacht wird. Heute gibt es mehrere tausend semisynthetische Derivate, deren Wirkspektrum, Stabilität und Verträglichkeit sehr viel besser sind als bei den Medikamenten der ersten Generation. ${ }^{12}$ Antibiotikapräparate auf $\beta$-Lactambasis erreichen ein geschätztes Weltmarktvolumen von zehn Milliarden US-Dollar jährlich. ${ }^{13}$ 


\section{2. „Marine“ Pilze und ihre Sekundärmetaboliten}

Bei der Suche nach neuen Naturstoffen stieß man immer wieder auf bekannte Verbindungen. Um diesem Problem zu entgehen, wurden verstärkt Organismen aus wenig untersuchten Habitaten bearbeitet. Dazu gehören Extremophile aus der Wüste, heißen Quellen und der Antarktis. ${ }^{14}$ Aber auch der marine Lebensraum ist zu einem interessanten Forschungsgebiet bezüglich neuer Wirkstoffe und Leitstrukturen geworden. ${ }^{15}$ Zwischen 1970 und 1997 wurden vorwiegend marine Makroorganismen und symbiontische Bakterien bearbeitet. ${ }^{16}$ Ab 1998 rückten auch Pilze mariner Herkunft ins Interessenfeld der Naturforscher ${ }^{17}$ und konnten ihr großes Potential bezüglich der Diversität an Sekundärmetaboliten unterstreichen.

Der Penicillium-Stamm (OUPS-79) zum Beispiel, der von der Meeresalge Enteromorpha intestinalis isoliert wurde, ist der Produzent der ungewöhnlichen, aus Aminosäuren abgeleiteten Communesine A $(\underline{\mathbf{5}})$ und B $(\underline{\mathbf{6}}){ }^{18} \underline{\mathbf{5}}$ zeigte hohe Aktivität $\left(E D_{50}=0.45 \mu \mathrm{g} / \mathrm{mL}\right)$ in einem P388 lymphozytischen Leukämie Test, während $\underline{\mathbf{6}}$ nur moderate Cytotoxizität aufwies $\left(E D_{50}=3.5 \mu \mathrm{g} / \mathrm{mL}\right)$.

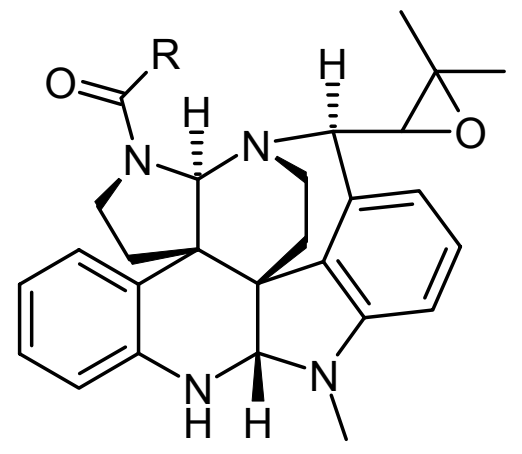

$\underline{\mathbf{5}} \mathrm{R}=\mathrm{CH}_{3}$

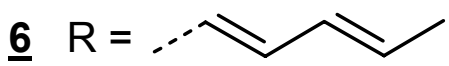

Eine weitere Studie des Penicillium-Isolates OUPS-79 führte zur Isolierung der Penochalasine A-H (ㅁ-14). ${ }^{19}$ Diese Verbindungen werden zur Familie der Cytochalasine gezählt und fielen ebenfalls durch ihre Cytotoxizität gegen P388 Leukämie-Zellen auf. Die höchste biologische Aktivität wiesen die Penochalasine

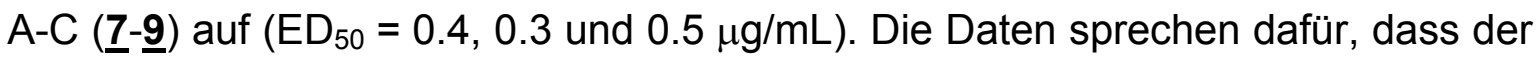
Pyrrolring ein wichtiges Strukturelement für die Cytotoxizität darstellt. Schon die Hydrierung des Fünfringes (10 $)$ verringert die Wirkung auf $\mathrm{ED}_{50}=3.2 \mu \mathrm{g} / \mathrm{mL}$. 


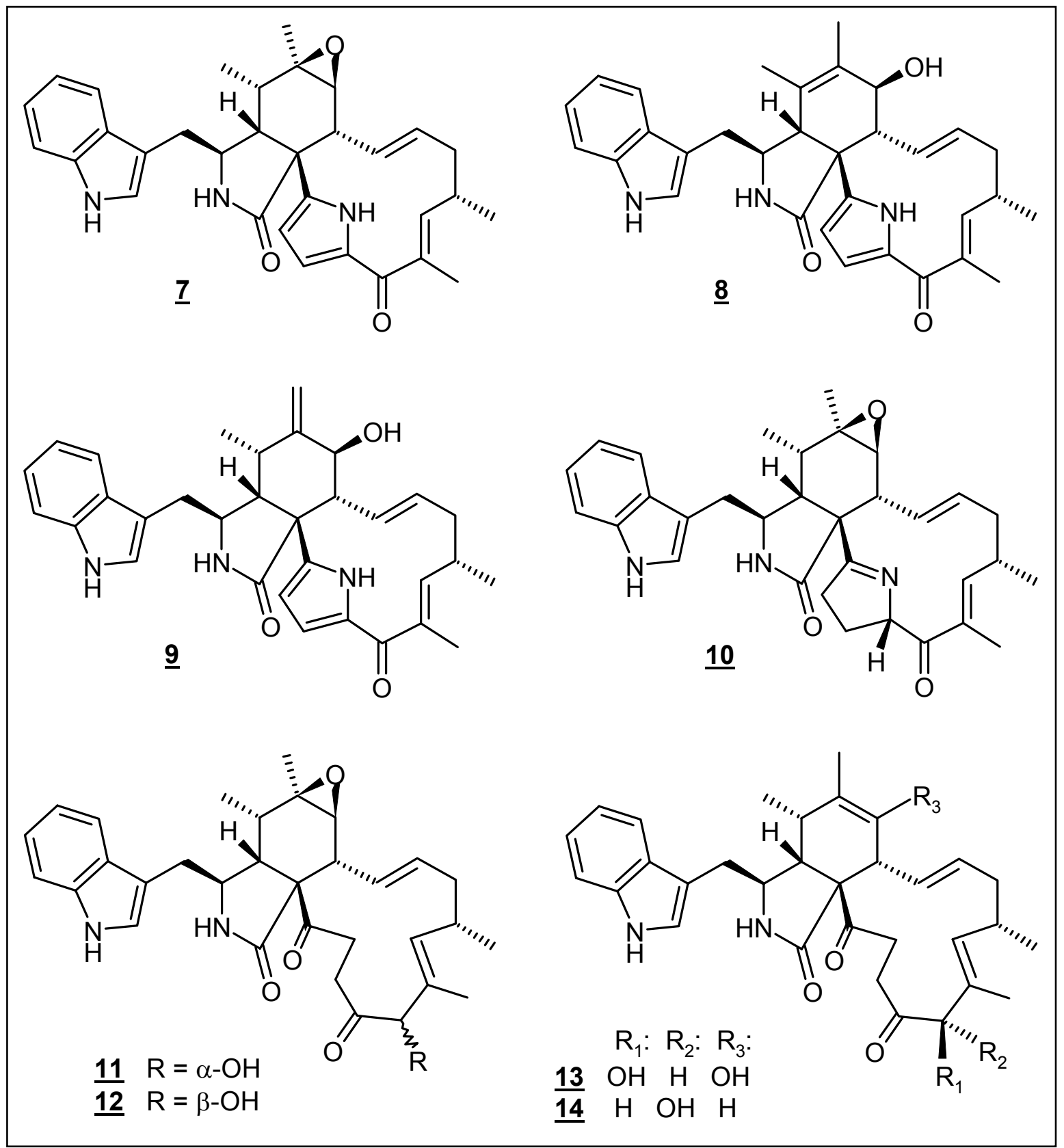

Abbildung 1: Penochalasine aus Penicillium sp. (OUPS-79).

Die Penostatine repräsentieren die dritte Klasse an neuen Metaboliten, die von der Arbeitsgruppe um A. NUMATA aus oben genanntem Stamm erhalten wurden. Die Penostatine A-D $(\underline{\mathbf{1 5}}-\underline{\mathbf{1 8}})^{20}$ sind charakterisiert durch ein tricyclisches Grundgerüst, von dem eine $\mathrm{C}_{9}$-Seitenkette abzweigt, während F-I ( $\left.\underline{\mathbf{2 0}}-\underline{\mathbf{2 3}}\right)^{21}$ durch zusätzliche Cyclisierungs-Reaktionen entstehen. Die absolute Stereochemie der Substanzen wurde über die Mosher-Methode und mit Hilfe von CD-Spektroskopie ermittelt. ${ }^{22}$ Die Aktivität der Penostatine wurde in einem P388 lymphozytischen Leukämie 
Testsystem evaluiert und bewegt sich im Bereich von 0.5 bis $11.0 \mu \mathrm{g} / \mathrm{mL}$. Penostatin E (19), die einzige Verbindung, die nicht aus einem tricyclischen Kern besteht, zeigte dabei die geringste Wirkung. Es ist schon bemerkenswert, dass strukturell so verschiedene Sekundärmetabolite von einem Pilzstamm ausgeschieden werden und dabei über eine vergleichbare cytotoxische Potenz verfügen.

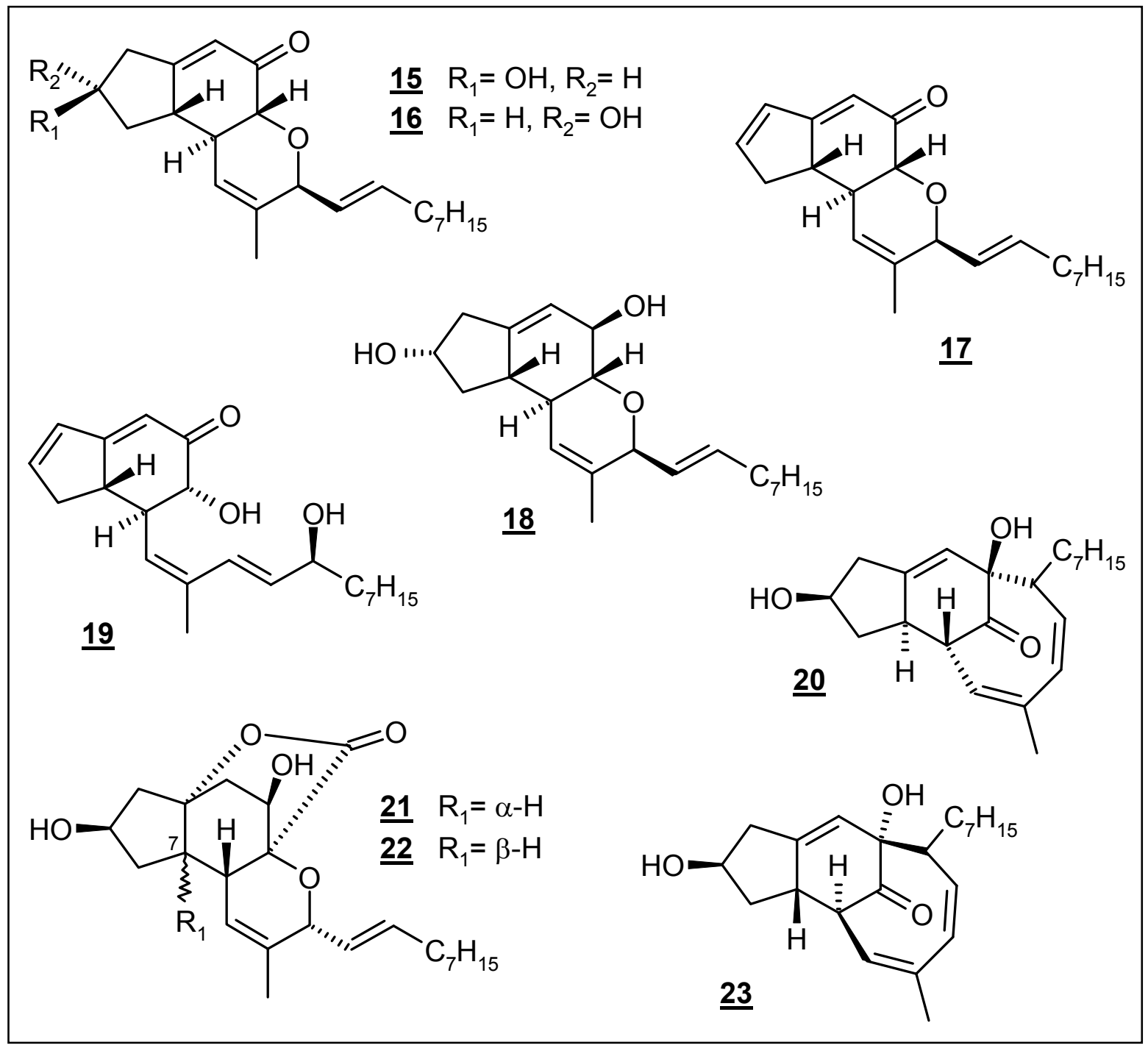

Abbildung 2: Penostatine aus Penicillium sp. (OUPS-79).

Mittlerweise sind 272 neue Metabolite aus marinen Pilzen bekannt. ${ }^{23}$ Die Pilzisolate stammen aus Sedimentproben, Holzsubstrat, Schwämmen, Algen, Bryozoen und anderen Makroorganismen. Aus Schwämmen wurden nicht nur die größte Anzahl unterschiedlicher Pilzgattungen isoliert, mit 28\% stellen sie auch die 
ergiebigste Quelle an neuen Substanzen dar. ${ }^{23}$ Die biologische Aktivität der Pilzmetabolite reicht über antibiotische und anticancerogene Eigenschaften, beinhaltet aber auch selektive Aktivitäten wie Zellzyklus-Inhibierung, antivirale Aktivität, neuritogene Aktivität, Phosphatase- und Kinase-Inhibierung und die Wirkung als Radikalfänger. ${ }^{17}$

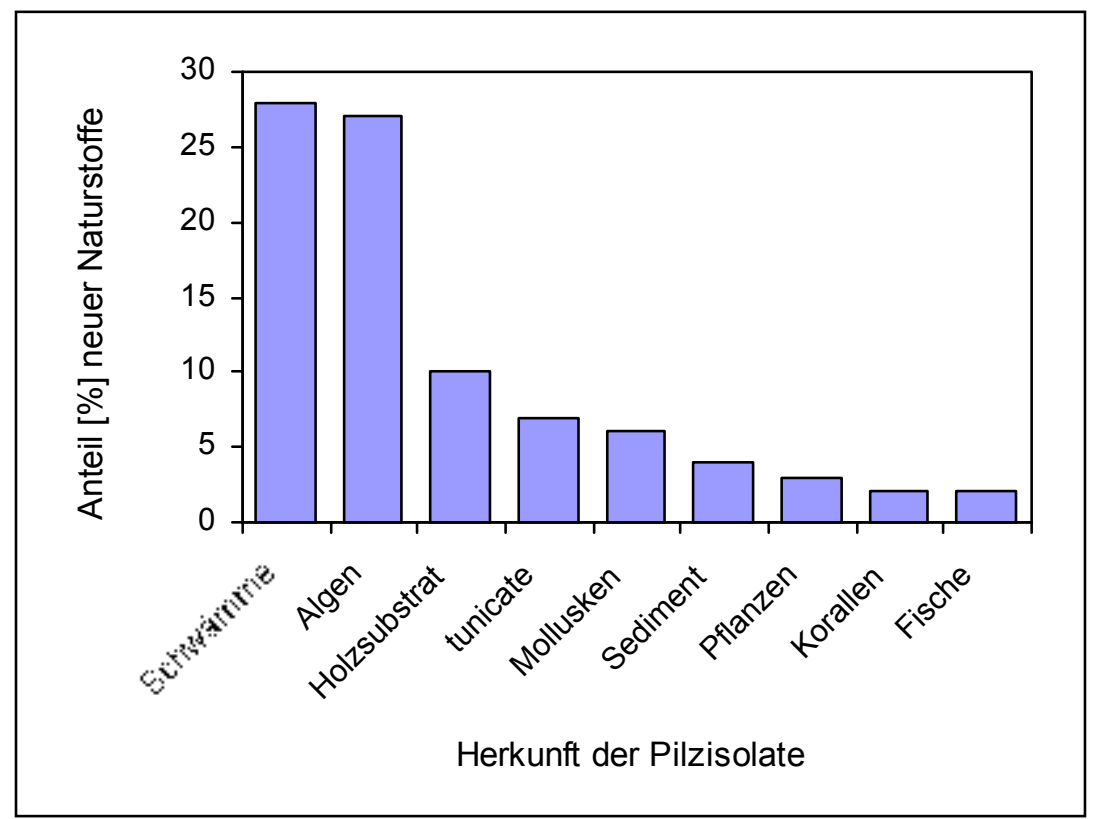

Abbildung 3: Die Verteilung publizierter neuer Naturstoffe aus marinen Pilzen in Abhängigkeit der Pilzquelle. ${ }^{22}$

Eine Weichkoralle, im Meer vor Vancouver Island (Canada) gesammelt, war der Wirt eines nicht identifizierten Pilzstammes LL37H248. Die Kultivierung des Pilzes führte zur Isolierung von Spiroxin A (24) und vier weiteren neuen Spirobisnaphthalenen mit octacyclischem Ringsystem. ${ }^{24}$ Neben seiner Cytotoxizität $\left(\mathrm{IC}_{50}=90 \mathrm{ng} / \mathrm{mL}\right)$ wies $\underline{\mathbf{2 4}}$ eine antibakterielle Wirkung auf und war aktiv in einem Maus-Model gegen menschliche Eierstock-Krebszellen.

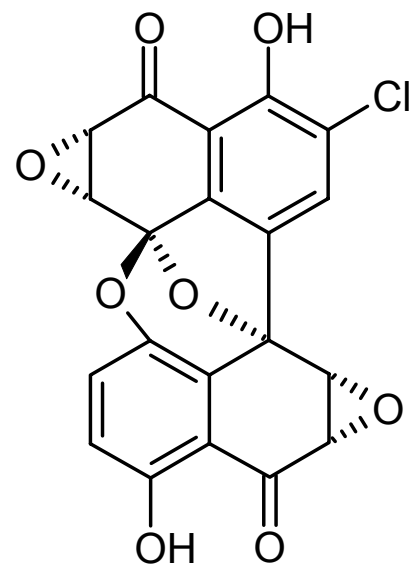


Auch Pilze der Gattung Xylarium sind eine ergiebige Quelle bioaktiver Metabolite. ${ }^{25}$ Der Stamm \#2508 aus dem Südchinesischen Meer ist der Produzent eines neuen cyclischen Peptides, welches eine Zimtsäure-Allenether-Seitenkette enthält. ${ }^{26}$ Neben dem Xyloallenolid A (25) wurden auch der But-2,3-dienylether

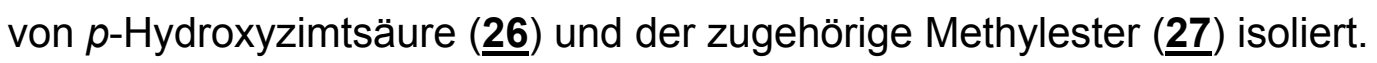

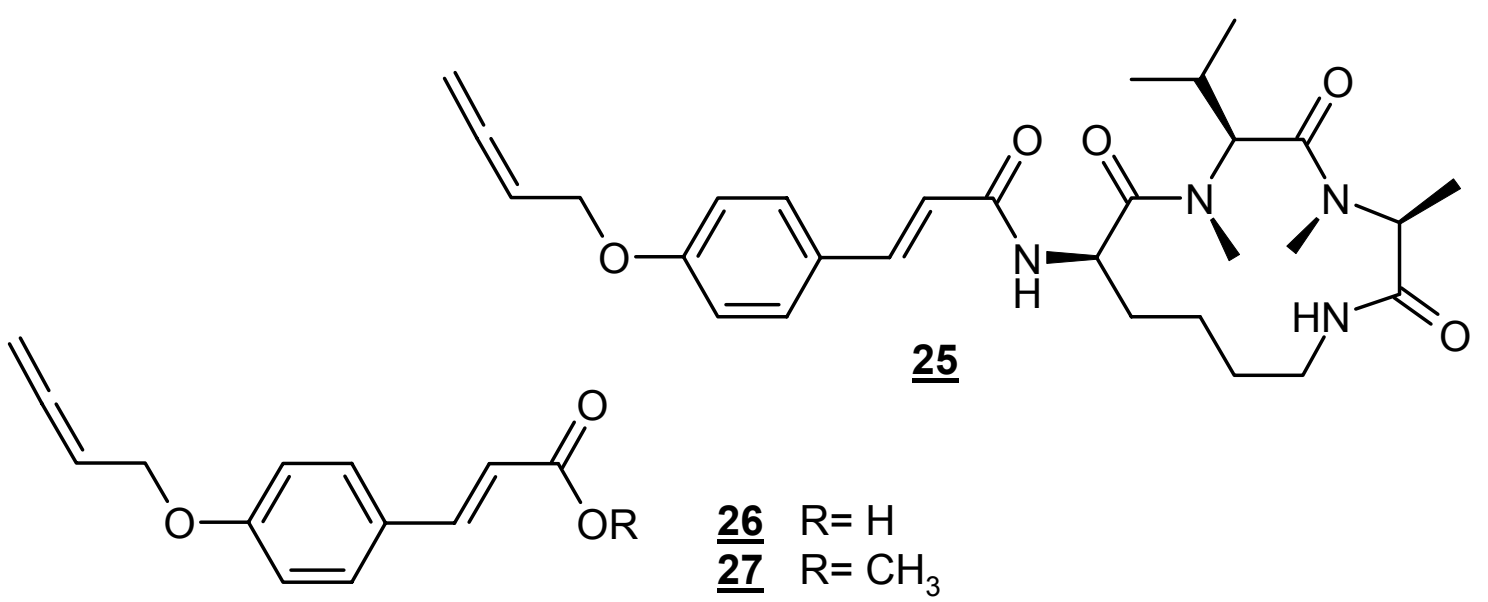

Wird ein Pilz von einem marinen Organismus isoliert, zeigt dies nicht notwendigerweise, dass der Pilz dort auch wächst. Ebenso könnte eine Spore aus dem terrestrischen Millieu von einem Makroorganismus aus dem umgebenen Wasser filtriert worden sein, die in der marinen Umgebung in ihrem Wirt geruht hat und erst beim Aufbringen auf ein Nährmedium wieder zum Auskeimen gebracht wurde. Aus diesem Grund wird das marine Ökosystem zum einen in die obligat marinen Pilze eingeteilt, für die das marine Millieu zum Wachsen und Sporulieren unerlässlich ist, während die fakultativ marinen Pilze eigentlich aus Süßwasser oder terrestrischen Habitaten stammen, aber auch im Stande sind, unter marinen Bedingungen zu überleben. ${ }^{27}$ Studien belegen, dass viele marine Pilzisolate ubiquitär verbreiteten terrestrischen Gattungen wie Aspergillus oder Penicillium angehören. ${ }^{28}$ Es ist allerdings auch möglich, dass morphologisch identische Stämme aus terrestrischen und marinen Habitaten sich den jeweiligen physiologischen Gegebenheiten angepasst haben und aus dem Grund einzigartige Sekundärmetaboliten bilden.

In diesem Zusammenhang stellt sich die Frage nach der ökologischen Funktion der Pilze im Meer. Zum einen wird innen eine wichtige Rolle bei der Zersetzung 
von abgestorbenem Pflanzenmaterial und der damit verbundenen Rückführung von Nährstoffen ins Ökosystem zugeschrieben. ${ }^{29}$ Zum anderen können von Pilzen ausgeschiedene Sekundärmetabolite der Abwehr dienen. Es wurde beobachtet, dass Teile der Grünalge Blidingia minima, die mit dem Ascomyceten Turgidosculum ulvae infiziert waren, von Invertebraten als Nahrung verschmäht wurden. ${ }^{27}$ SIEPMANN und HöNK diskutierten die ökologische Rolle einer Hefe, die in Schwämmen vorkommt, als Produzent von Vitaminen. ${ }^{30}$ Bislang wurde aber nur in einem Fall von einer echten Symbiose zwischen Pilz und Schwamm berichtet. Spezien der Gattung Koralionastes wurden immer in Assoziation mit Schwämmen vorgefunden und es wird angenommen, dass in oder auf dem Wirt die Entwicklung der Ascosporen erfolgt. ${ }^{31}$

Mikroorganismen produzieren Sekundärmetabolite aus spezifischen Gründen, die wir meistens nicht kennen, aber annehmen, dass sie die Überlebenschancen des Produzenten in der Umwelt erhöhen. ${ }^{32}$ Die Produktion antibiotischer Substanzen ist demnach neben Umweltfaktoren auch von der Anwesenheit konkurrierender Mikroorganismen abhängig. Es wird vermutet, dass die Biosynthesewege bestimmter Sekundärmetabolite über Faktoren reguliert werden, die mit der Wahrnehmung mikrobieller Konkurrenten zusammenhängen. SONNENBICHLER et al. konnten zeigen, dass die Synthese einiger Metaboliten auf das 400-fache erhöht werden kann, wenn der Produzent in Gegenwart eines Antagonisten kultiviert wird. ${ }^{33}$ JENSEN und FENICAL konnten bei 12 von 100 getesteten marinen Pilzstämmen die Bildung neuer aktiver Metabolite durch Co-Fermentationen mit

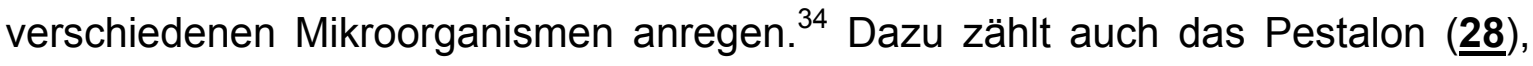
das erstmalig in einer Mischfermentation einer Pestalotia sp. (Stamm CNJ-328), isoliert von der Oberfläche der Braunalge Rosenvingea sp., mit einem einzelligen marinen Bakterium auftrat. In Kontrollversuchen, in denen der Pilz und das Bakterium separat kultiviert wurden, wurde keine Bildung von $\underline{\mathbf{2 8}}$ verzeichnet. Weder die Zugabe des organischen Extraktes noch der zellfreien Kulturbrühe des Bakteriums konnte die Antibiotika-Produktion des Pilzstammes beeinflussen. Diese Beobachtungen ließen den Schluß zu, dass die Regulation der PestalonProduktion nicht chemisch vermittelt wird. Interessanterweise konnten geringe Pestalon-Mengen isoliert werden, nachdem der Pestalotia-Stamm einer Stressfermentation durch Zugabe von $1 \%$ Ethanol ausgesetzt wurde. 


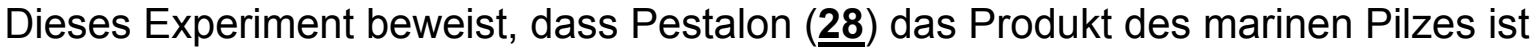
und seine Biosynthese über externe Faktoren gesteuert wird.<smiles>COc1c(Cl)c(C)c(Cl)c(O)c1C(=O)c1c(O)c(O)cc(O)c1CC=C(C)C</smiles>

Die Struktur von $\underline{\mathbf{2 8}}$ wurde mit spektroskopischen Methoden zu einem chlorierten Benzophenon aufgeklärt und über eine Röntgenkristall-Analyse bestätigt. $^{35}$ Pestalon (28) zeigte moderate in vitro Cytotoxizität gegen verschiedene Tumorzell-Linien, viel interessanter ist aber die antibiotische Aktivität gegen Methicillinresistente Staphylococcus aureus (MIC $=37 \mathrm{ng} / \mathrm{mL}$ ) und Vancomycin-resistente Enterococcus faecium (MIC $=78 \mathrm{ng} / \mathrm{mL})$.

Zusammenfassend kann man sagen, dass viele der marinen Pilze nach morphologischen Gesichtspunkten den terrestrischen Pilzen ähneln oder mit ihnen identisch sind. Dennoch sind sie in der Lage, im marinen Lebensraum zu wachsen und vielleicht wird gerade durch die marine Umgebung die Produktion an Sekundärmetabolite in besonderer Weise beeinflusst. Zahlreiche Publikationen zeigen, dass die Erforschung "mariner" Pilze zu aufregenden neuen chemischen Strukturen geführt hat, die eine Vielzahl an biologischen Aktivitäten aufweisen und potentielle Kandidaten für eine Medikamenten-Entwicklung sind. 


\section{Aufgabenstellung}

Im Mittelpunkt dieser Arbeit stand die Untersuchung von „marinen“ Mikroorganismen bezüglich ihre Naturstoffproduktion.

Dipl.-Biol. M. HAIN (Institut für Geomikrobiologie, Universität Oldenburg) stellte dem Arbeitskreis ZeECK Pilzisolate zur Verfügung, isoliert von Bryozoen aus der Nordsee. Ein weiteres marines Pilzisolat stammt von Dipl.-Biologin H. STEVENS (Universität Oldenburg).

Die Pilzstämme sollten einem chemischen Screening unterzogen und nur die im Screening auffälligen Stämme weiter kultiviert und hinsichtlich der Sekundärmetabolite bearbeitet werden.

Nach der Isolierung unter Verwendung verschiedener chromatographischer Trennverfahren sollte sich die Strukturaufklärung unter Einbeziehung moderner chemischer und spektroskopischer Methoden anschließen.

Bei Stämmen mit interessantem Metabolitenmuster sollte durch Variation der Kultivierungsbedingungen (OSMAC = One-Strain-many-Compounds $)^{36}$ die biosynthetische Leistungsfähigkeit der Produzenten überprüft werden.

Mit allen Naturstoffen dieser Arbeit sollten je nach Perspektive weitere Untersuchungen zur chemischen Reaktivität (Derivatisierung), biologischen Aktivität (Plattendiffusionstest, Einschleusung in Testsysteme) und Biogenese (Fütterungsexperimente mit markierten Verbindungen) durchgeführt werden. 


\section{Chemisches Screening}

\subsection{Screening}

Bei der Suche nach neuen Naturstoffen können unterschiedliche Screeningstrategien verfolgt werden. Von großer Bedeutung sind hierbei das Biologische und das Chemische Screening.

Das auf F.A. WAKSMAN ${ }^{37}$ zurückgehende Biologische Screening zielt darauf ab, selektiv Substanzen mit bestimmten biologischen Eigenschaften zu finden. Mit Hilfe von Plattendiffusionstests prüft man Rohextrakte oder bereits fraktionierte Extrakte auf ihre antibiotische Aktivität gegen verschiedene Testorganismen. Die Komponenten, die sich als wirksam erweisen, werden anschließend mit geeigneten Methoden aufgereinigt und in ihrer Struktur aufgeklärt. Nachteilig bei diesem Screeningansatz ist die Abhängigkeit von bestimmten Testsystemen. Wirkstoffe, die in den Tests nicht ansprechen, werden auf diesem Weg nicht weiter verfolgt. Außerdem kann es zu falsch positiven Ergebnissen aufgrund von synergistischen Effekten im Extrakt kommen. Viele Naturheilmittel sind komplexe Substanzgemische und entfalten ihre Wirkung nur im synergistischen oder antagonistischen Wechselspiel mit anderen Bestandteilen eines Rohextraktes. So wird der Extrakt der Mistel, der verschiedene Viscotoxine, Polysaccharide und Lectine enthält, erfolgreich in der Krebstherapie eingesetzt. Die aufgereinigten Einzelkomponenten sind hingegen deutlich weniger wirksam als der Rohextrakt. ${ }^{38}$ Es ist ferner problematisch, von Rohextrakten oder deren Fraktionen Bibliotheken anzulegen, weil kleinste Veränderungen der Extrakte zu falschen Aussagen führen können.

Das zunehmende Verständnis von Krankheiten auf genetischer und molekularer Ebene führte zum Target-orientierten Screening, ${ }^{39}$ bei dem Enzym- und RezeptorAssays Eingang in die Tests finden und so gezielt auf einzelne Krankheiten und pharmazeutische Anwendungen getestet werden kann. Dies geschieht heute in der Industrie im High-Throughput-Screening (HTS). ${ }^{40}$ Die modernen Testverfahren sind automatisiert, werden im Kleinstmaßstab auf Mikrotiterplatten durchgeführt und bewältigen auf diese Weise große Substanzbibliotheken oder zahlreiche Extraktproben binnen kürzester Zeit. 
Während beim Biologischen Screening eine biologische Aktivität von Extrakten Ausgangspunkt ist, werden im Chemischen Screening Metabolite isoliert, die durch ihre physikochemischen Eigenschaften auffallen. Dieses Verfahren wurde erstmals 1970 von H. UMEZAWA ${ }^{41}$ beschrieben, und in den Arbeitsgruppen von H. ZÄHNER ${ }^{42}$ und A. ZEECK ${ }^{43}$ weiterentwickelt. Das Chemische Screening hat zum Ziel, möglichst alle Substanzen einer Extraktprobe zu isolieren und identifizieren. Hierbei erfolgt eine chromatographische Auftrennung der Rohprodukte. Zur Detektion der Metabolite dient die Eigenfarbe, UV-Löschung und die Anfärbbarkeit mit verschiedenen Sprühreagenzien. Die $R_{\mathrm{f}}$-Werte helfen bei der Bildung von Untergruppen bezüglich der Polarität der Substanzen. Erst nach Isolierung, Strukturaufklärung und Charakterisierung der Reinsubstanzen erfolgt eine möglichst breite biologische Austestung. Dieses Verfahren hat den Vorteil, eine Vielzahl von neuen Naturstoffen zugänglich zu machen, auch wenn ihre biologische Wirkung noch nicht bekannt oder mit herkömmlichen Testsystemen nicht nachweisbar ist. Ebenso erhält man auf diese Weise Wirkstoffe, die im biologischen Screening durch bekannte Substanzen überdeckt werden oder so niedrig konzentriert sind, dass sie keine Wirkung zeigen. Ein Nachteil des chemischen Screenings ist die nur semi-automatisierbare Durchführbarkeit und der hohe Anteil nicht digitalisierbarer Daten. Das Screeningfenster liegt häufig auf den Farbreaktionen mit verschiedenen Sprühreagenzien, die sich nicht standardisiert beurteilen lassen. Zudem werden auch hier bekannte Verbindungen aufgefunden und können von neuen Naturstoffen nur mit viel Erfahrung unterschieden werden.

In den letzten Jahren sind auf dem Gebiet des chemischen Screening durch fortschreitende technische Entwicklungen neue Techniken entwickelt worden, um die Reisolierungsrate zu verringern. Mit Hilfe gekoppelter Methoden wie HPLCDAD ${ }^{44}$, HPLC-MS ${ }^{45}$ oder HPLC-NMR ${ }^{46}$ können bekannte, bereits in Datenbanken beschriebene Metabolite schneller identifiziert werden.

Ein Ziel der Weiterentwicklung der Methoden ist es, eine festgestellte biologische Aktivität bestimmten physikochemischen Parametern zuzuordnen. Nur mit dem Verständnis des Wechselspiels zwischen Wirkmolekülen und Wirkort können neue Leitstrukturen entwickelt werden, die den Weg zum spezifisch wirkenden Arzneimittel eröffnen. 


\subsection{Durchführung des Chemischen Screenings}

Um ein breites Spektrum an Nährmedienbestandteilen abzudecken, wurden die Stämme im Chemischen Screening in sechs verschiedenen Medien (je 1L 1158, E2, G20, 1405, 1549 und MB) kultiviert, angeimpft direkt von einer gut bewachsenen Agarplatte. Nach vier bzw. sechs Tagen wurde jeweils die Hälfte des Ansatzes geerntet. So konnten neben dem Metabolitenspektrum auch Aussagen zum Produktionsverlauf gemacht werden.

Die Aufarbeitung der Kulturen ist in Abbildung 4 dargestellt.

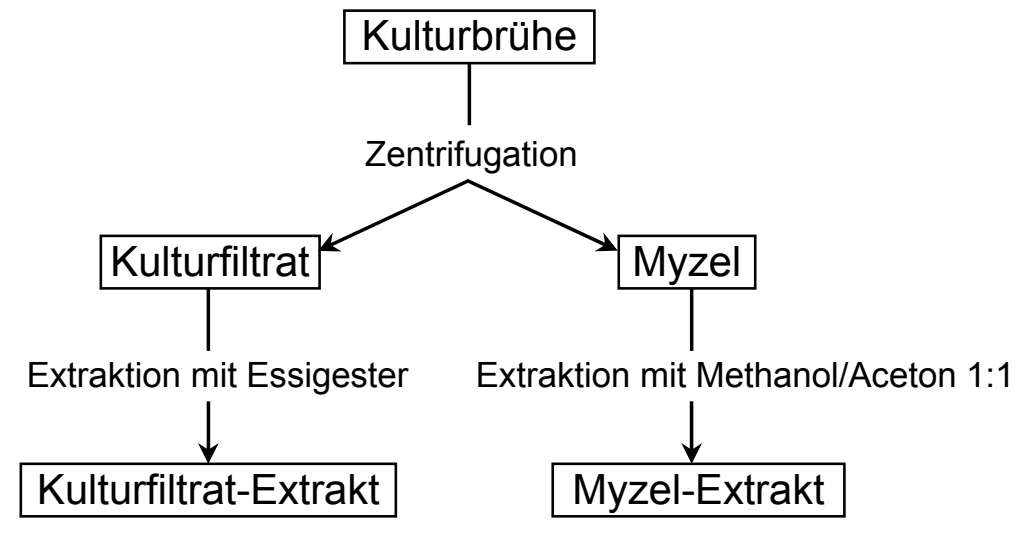

Abbildung 4: Aufarbeitungsschema der marinen Pilze.

Die erhaltenen Rohprodukte wurden in $2 \mathrm{~mL}$ Methanol/Wasser 5:1 aufgenommen und $5 \mu \mathrm{L}$ dieser Lösung dünnschichtchromatographisch untersucht. Dazu entwickelte man die betüpfelten Nano-DC-Platten in den Laufmittelsystemen Chloroform/Methanol 9:1 (CM91) und Eisessig/n-Butanol/Wasser 1:4:5, um einen möglichst großen Polaritätsbereich abzudecken. Die Visualisierung der Metabolite erfolgte durch Eigenfarbe, UV-Absorption bei 254 und $366 \mathrm{~nm}$ sowie durch Verwendung verschiedener Sprühreagenzien. Benutzt wurden Anisaldehyd/ Schwefelsäure (Anis; charakteristische Anfärbung von Zuckern, Steroiden, Terpenen), Vanillin/Schwefelsäure (VS; Alkohole, Phenole, Terpene), Orcin (Zucker) und Ehrlich (Amine, Furane). Hierdurch wird gewährleistet, dass eine große Bandbreite an Verbindungen mit unterschiedlichen Funktionalitäten aufgefunden wird. Zur Dokumentation wurden die Dünnschichtchromatogramme fotografiert. 


\subsection{Stammauswahl}

Die Pilzisolate stammen zum einen von Herrn Dipl.-Biol. M. HAIN (Universität Oldenburg). Sie wurden von verschiedenen Bryozoen isoliert, die der Nordsee entnommen wurden.

\begin{tabular}{|c|c|c|}
\hline \multirow{4}{*}{ Bryozoe } & $\begin{array}{c}\text { Pilzisolat } \\
\text { Stamm-Nr. }\end{array}$ & $\begin{array}{c}\text { Gattung } \\
\text { (ITS1- bzw. 18S-rDNA- } \\
\text { Sequenzierung)* }\end{array}$ \\
\hline \multirow{5}{*}{ Electra pilosa } & WDMH2 & Hypomyces sp. \\
\cline { 2 - 3 } & WDMH21 & Coccodinium sp. \\
\cline { 2 - 3 } & WDMH22 & Phialophora malorum \\
\cline { 2 - 3 } & WDMH24 & Penicillium coralligerum \\
\cline { 2 - 3 } & WDMH45 & Paraspheaoshaeria sp. \\
\cline { 2 - 3 } & WDMH46 & Paraspheaoshaeria sp. \\
\cline { 2 - 3 } & WDMH47 & Chaetomium sp. \\
\cline { 2 - 3 } Alcyonidium & WDMH51 & Aspergillus sp. \\
\hline \multirow{4}{*}{ gelatinosum } & WDMH28 & nicht identifiziert \\
\cline { 2 - 3 } & WDMH35 & Aspergillus fumigatus \\
\cline { 2 - 3 } & WDMHC2 & Fusarium sp. \\
\hline
\end{tabular}

Tabelle 1: Liste der von Dipl. Biol. M.HAIN überlassenen Pilzisolate.

Zum anderen isolierte Dipl.-Biologin H. STEVENS (Universität Oldenburg) die marine Pilzkultur WDSH, die taxonomisch nicht bestimmt wurde. Der Stamm fiel dadurch auf, dass er eine Substanz mit pinker Eigenfarbe in den Nährstoffagar abgab.

Die zwölf Stämme wurden, wie in Kapitel 3.2. beschrieben, dem chemischen Screening unterzogen. Die Ergebnisse sind in Tabelle 2 zusammengefasst.

Auswahlkriterien für eine weitere Bearbeitung waren Anzahl und Intensität interessanter Zonen auf dem angefärbten Dünnschichtchromatogramm (DC). Über die Intensität der Farbreaktionen mit den Sprühreagenzien und der UV-Absorbtion kann auf die Menge gebildeter Sekundärstoffe geschlossen werden. Von Interesse ist das Vorliegen von zwei bis drei Hauptmetaboliten, was eine gute Detektion und 
zügige Isolierung ermöglicht. Durch das Anfärbeverhalten sollte in Grenzen auch ein Rückschluß über darin enthaltene funktionelle Gruppen erlaubt sein. Die Variabilität eines Stammes kann über die Veränderung des Metabolitenspektrums bei Nährmedienwechsel eingeschätzt werden. Letzten Endes ist die Bewertung eines Stammes aber durchaus subjektiv und von Erfahrungswerten geprägt.

Aufgrund oben beschriebener Kriterien wurden die Stämme WDMH24, WDMH28, WDMH35, WDMH46, WDMH51 und WDMHC2 als interessant eingestuft und für eine weitere Bearbeitung ausgewählt. In den folgenden Kapiteln werden Isolierung und Strukturaufklärung von Metaboliten aus diesen Stämmen beschrieben.

\begin{tabular}{|c|c|c|}
\hline Stamm & $\begin{array}{c}\text { Metaboliten- } \\
\text { spektrum }\end{array}$ & Variabilität \\
\hline WDMH2 & +++ & ++ \\
\hline WDMH21 & + & + \\
\hline WDMH22 & + & + \\
\hline WDMH24 & ++ & ++ \\
\hline WDMH28 & ++++ & ++ \\
\hline WDMH35 & +++++ & +++++ \\
\hline WDMH45 & +++ & ++ \\
\hline WDMH46 & ++++ & +++ \\
\hline WDMH47 & +++ & +++ \\
\hline WDMH51 & +++ & ++++ \\
\hline WDMHC2 & ++++ & ++ \\
\hline WDHS & + & + \\
\hline
\end{tabular}

Tabelle 2: Bewertung der bearbeiteten Pilzstämme basierend auf dem Metabolitenspektrum und der Variabilität bei Nährmedienwechsel; (+++++ [sehr gut] bis + [kaum vorhanden]). 


\section{Stämme mit literaturbekannten Metaboliten}

\subsection{WDMH35}

Der Aspergillus-Stamm WDMH35 fiel im chemischen Screening vor allem durch die Farbreaktion seiner Metabolite im Dünnschichtchromatogramm (DC) auf, z.B. waren mit Anis neben einem günen noch bräunliche, gelbe, pinke und lila Zonen zu sehen. Die Metabolitenproduktion war nach sechs Tagen deutlich höher als nach vier Tagen. Der Intensität dem DC nach zu urteilen, lagen diese auch in ausreichender Menge für eine Isolierung und Strukturaufklärung vor.

\subsubsection{Isolierung und Charakterisierung der Metabolite}

Sowohl der Kulturfiltrat- als auch der Myzel-Extrakt aus einer 5 L-Fermentation (NL G20, $28^{\circ} \mathrm{C}, 120$ spm, sechs Tage) wurden einer Säulenchromatographie an Kieselgel mit einem Dichlormethan/Methanol-Gradienten unterzogen. Weitere Aufarbeitungsschritte unter Verwendung von Kieselgel und Sephadex LH-20 (Abbildung 5) ergaben drei Verbindungen als Reinsubstanz.

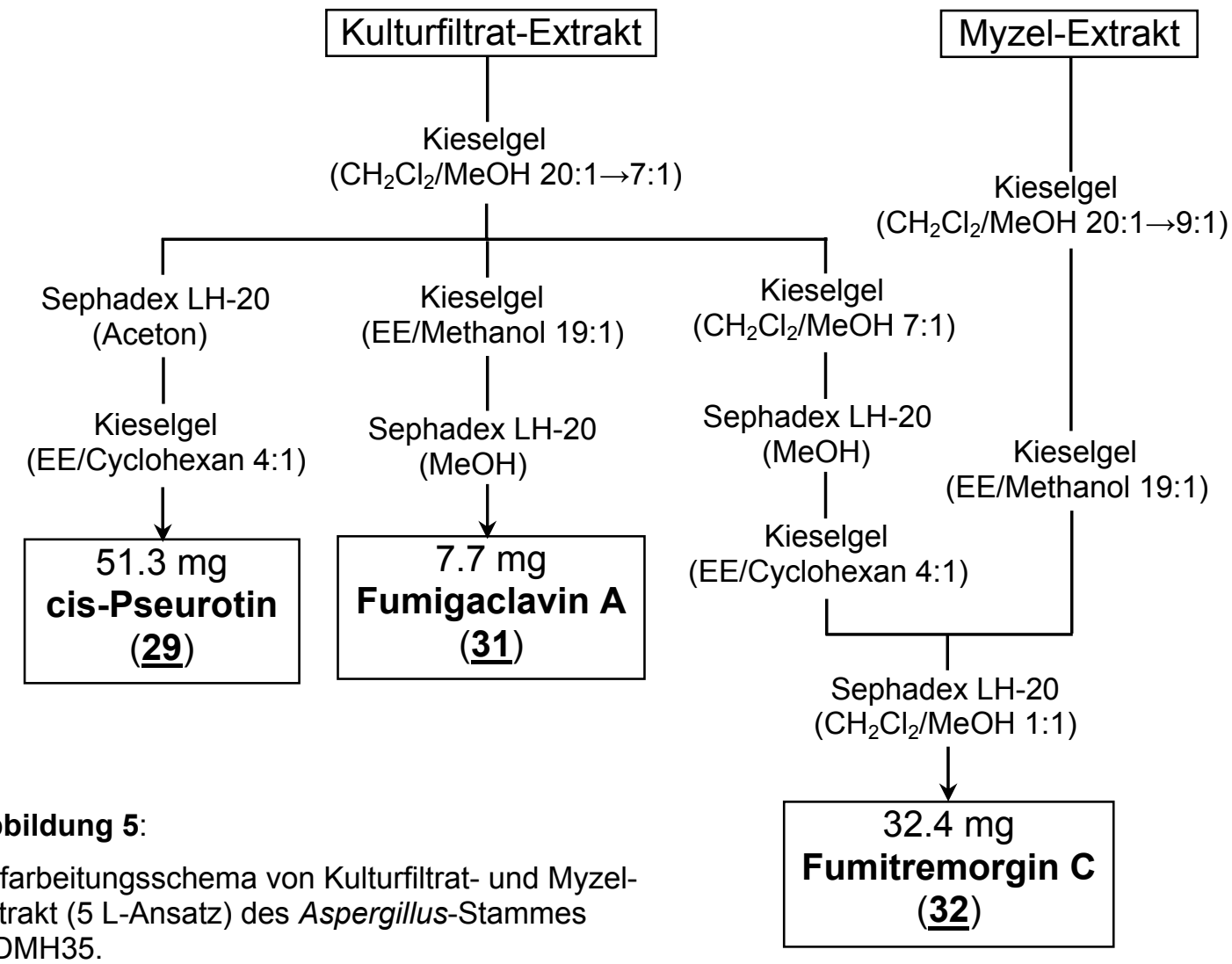
WDMH35. 


\section{cis-Pseurotin $(\underline{29})$}

$\underline{29}$ stellt die Hauptkomponente des Stammes WDMH35 dar, liegt als farbloses ÖI vor und färbt mit Anis gelbbraun, mit Orcin hellbraun und mit Ehrlich grünlich an. Aus dem ESI-Massenspektrum ergab sich eine Masse von $431 \mathrm{~g} / \mathrm{mol}$. Die ungerade Molmasse deutet auf die Anwesenheit einer ungeraden Anzahl von Stickstoffatomen hin. Eine schwache Bande im IR-Spektrum bei $1544 \mathrm{~cm}^{-1}$ bestätigt das Vorliegen einer $\mathrm{NH}-$ Gruppierung, ebenso weist es zwei Carbonylbanden bei 1722 und $1703 \mathrm{~cm}^{-1}$ auf, deren Resonanzen durch Konjugation erniedrigt oder durch Ringspannung bzw. elektronegative Atome erhöht erscheinen. Das ${ }^{13}$ C-NMR-Spektrum zeigt 22 Kohlenstoffatome, darunter zwei Ketogruppen bei $\delta_{\mathrm{C}}=196.6$ und 195.3 und ein Sauerstoff-substituiertes $\mathrm{sp}^{2}$-Zentrum bei $\delta_{C}=169.1$. Im Protonenspektrum sind im Hochfeldbereich zwei Methylgruppen zu beobachten. Das Signal bei $\delta_{H}=1.61$ liegt als Singulett vor, das Triplett bei $\delta_{\mathrm{H}}=0.92 \mathrm{mit} J=7.5 \mathrm{~Hz}$ bildet den Anfang eines $\mathrm{C}_{6}$-Spinsystems, welches neben einer cis-Doppelbindung $\left(J=10.5 \mathrm{~Hz}, \delta_{\mathrm{H}}=5.46,5.61\right)$ zwei Hydroxylgruppen (br $\mathrm{s}$ bei $\delta_{H}=2.40$ und 3.64) beinhaltet. Des Weiteren treten außer der Resonanz einer Methoxygruppe $\left(\delta_{\mathrm{H}}=3.34\right)$ drei Signale im Bereich von $\delta_{\mathrm{H}}=7.39$ bis 8.29 mit der Intensität von fünf Protonen auf, die sich aufgrund des Kopplungsmusters einem Phenylring zuordnen lassen.

Mit den ermittelten Strukturmerkmalen wurde eine Suche in $\underline{\text { Antibase }}^{47}$ durchgeführt. Man erhielt als Lösungsvorschlag cis-Pseurotin, das als Metabolit von Aspergillus fumigatus ${ }^{48}$ und Pseudeurotium ovalis ${ }^{49}$ beschrieben ist. Durch Literaturvergleich $^{50}$ konnte der Vorschlag bestätigt werden. Aufgrund des Drehwertes und der Herkunft dieser Substanz aus einer Aspergillus Spezies kann auch die Konfiguration als gesichert angesehen werden.

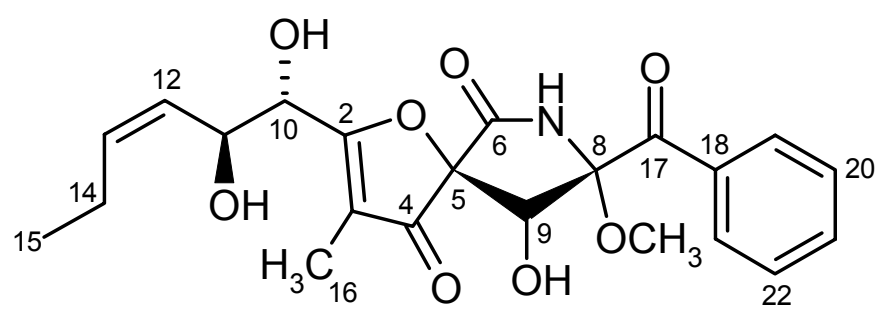


Der Metabolit erweist sich unter biologisch-medizinischen Gesichtspunkten als hoch interessant. Von den Pseurotinen, die auch als natürliches trans-Isomer vorkommen, ${ }^{51}$ wurde bisher über cytotoxische Effekte bei Eierstock-Krebszellen, ${ }^{52}$ Wirkungen als Wachstumsfaktor bei Nervenzellen, ${ }^{53}$ inhibitorische Aktivität bei der Chitinsynthase ${ }^{54}$ und psychotherapeutische Wirkungen durch Antagonismus zum Apomorphin ${ }^{48}$ berichtet.

\section{Fumigaclavin A (1ㅜ)}

Die farblose Substanz ist gut in Chloroform, besser noch in Methanol löslich, färbt mit Anis pink an und schleift auf dem Dünnschichtchromatogramm. Die Masse wurde durch El-MS zu $298 \mathrm{~g} / \mathrm{mol}$ bestimmt. Im ${ }^{13} \mathrm{C}-\mathrm{NMR}-S p e k t r u m$ treten Signale für drei Methyl-, zwei Methylen- und acht Methingruppen auf, von denen vier aliphatischer und vier aromatischer bzw. olefinischer Natur sind. Ferner sind fünf quartäre Kohlenstoffatome erkennbar, die Verschiebung bei $\delta_{c}=172.6$ weist auf das Vorhandensein einer Esterfunktion hin. Das Singulett bei $\delta_{H}=1.82 \mathrm{im}{ }^{1} \mathrm{H}$ NMR-Spektrum legt dar, dass es sich um eine O-Acetylgruppe handelt. Die Aufspaltung des Methylsignals bei $\delta_{H}=1.32$ zum Dublett vom Dublett zeigt zum einen mit $J=7.0 \mathrm{~Hz}$ die Nachbarschaft zur Methingruppe bei $\delta_{H}=2.06$ an, zum anderen die Fernkopplung mit $J=1.5 \mathrm{~Hz}$ zur Sauerstoff-substutuierten $\mathrm{CH}$-Gruppe bei $\delta_{\mathrm{H}}=5.63$. Das Spinsystem kann durch $\mathrm{H}, \mathrm{H}-\mathrm{COSY}-$ Kopplungen vervollständigt werden. Auf der einen Seite ist eine Methylengruppe $\left(\delta_{H}=2.69\right)$ gebunden, auf der anderen Seite wird die aliphatische Kette durch zwei Methingruppen $\left(\delta_{\mathrm{H}}=3.30,2.61\right)$ und eine diastereotop aufgespaltene $\mathrm{CH}_{2}$-Gruppe $\left(\delta_{\mathrm{H}}=3.42\right.$, 2.62) ergänzt. Im Tieffeldbereich des Protonenspektrums sind weitere Signale von vier Protonen zu erkennen, von denen drei aufgrund der Kopplungskonstanten einen 1,2,3-trisubstituierten Benzolring bilden, während das Signal des vierten aromatischen Protons als Singulett erscheint.

Bei einer Datenbanksuche in $\underline{\text { Antibase }}^{47}$ mit den ermittelten Strukturelementen stieß man auf zwei Verbindungen, die eine entgegengesetzte Stereochemie an den Positionen 8 und 9 aufweisen. ${ }^{55}$ Isofumigaclavin A $(\underline{\mathbf{3 0}})$ konnte aus verschiedenen Penicillium-Stämmen isoliert werden $^{56,57}$ und inm wurde eine bakteriostatische Wirkung auf gram-positive Bakterien nachgewiesen. ${ }^{58}$ Ebenso wies $\underline{30}$ in Tierversuchen eine Vielfalt schwacher pharmakologischer Wirkungen 
auf, z.B. wirkt es muskelrelaxierend, antidepressiv und lokalanästhetisch. ${ }^{59}$ Die weißen Kristalle schmelzen um $190^{\circ} \mathrm{C}$ und weisen in Methanol einen Drehwert von $[\alpha]_{D}^{20}=-54^{\circ}$ auf. Das Stereoisomer trägt den Namen Fumigaclavin A ( $\left.\underline{\mathbf{3 1}}\right)$ und stammt aus Aspergillus fumigatus. ${ }^{60}$ Die optische Aktivität ist durch einen ähnlichen Drehwert von $[\alpha]^{20}{ }_{D}=-57^{\circ}$ (in $\mathrm{CHCl}_{3}$ ) gekennzeichnet, der Schmelzpunkt ist mit $84^{\circ} \mathrm{C}$ angegeben. ${ }^{61}$ Die Substanzen zählen zur Klasse der Mutterkornalkaloide.
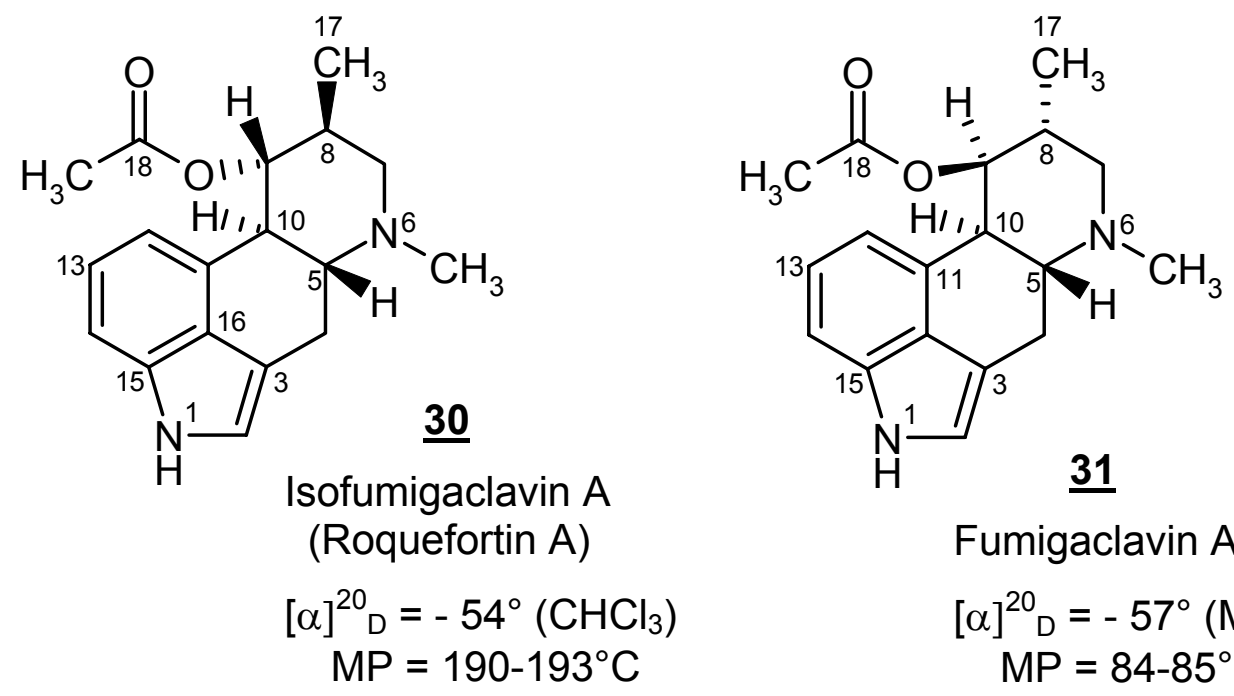

Fumigaclavin A

$$
\begin{aligned}
{[\alpha]^{20} } & =-57^{\circ}(\mathrm{MeOH}) \\
\mathrm{MP} & =84-85^{\circ} \mathrm{C}
\end{aligned}
$$

Der gravierende Unterschied der Schmelztemperatur beider Isomere könnte als geeignetes Charakteristikum dienen. Aufgrund der öligen und nicht kristallinen Konsistenz des Isolates konnte auf eine Schmelzpunktanalyse aber nicht zurückgegriffen werden. Auch der Drehwert der isolierten Substanz mit $[\alpha]_{D}^{20}=-47^{\circ}$ in methanolischer Lösung hilft nicht bei der Entscheidung, welches der Stereoisomere vorliegt.

In einem Artikel von $1978^{62}$ wurde die absolute Stereochemie von Isofumigaclavin A ( $\underline{\mathbf{3 0}})$ durch Röntgenstrukturanalyse veröffentlicht und mit der von Fumigaclavin A $(\underline{\mathbf{3 1}})^{63}$ verglichen. Die Methylgruppe an C-8 und die Acetylgruppe an C-9 stehen equatorial zueinander, die benachbarten Protonen koppeln mit $J=9.8 \mathrm{~Hz}$ und sind axial zueinander angeordnet. Das H-9-Signal der isolierten Substanz ( $\underline{\mathbf{x}}$ ) spaltet im ${ }^{1} \mathrm{H}$-Spektrum zum Dublett vom Dublett mit $J=1.5,1.5 \mathrm{~Hz}$ auf, wodurch eine axiale Konformation für dieses Proton ausgeschlossen werden kann. 
Die Reorientierung der 8-Methylgruppe und des 9-Acetoxysubstituenten von der axialen in eine äquatoriale Konformation zieht eine Tieffeldverschiebung für die Kohlenstoffatome 5, 7, 8, 9 und 10 mit sich. Ein in $\mathrm{CDCl}_{3}$ aufgenommenes ${ }^{13} \mathrm{C}$ NMR-Experiment von $\underline{\mathbf{x}}$ zeigt Übereinstimmung mit $\underline{\mathbf{3 1}}$ und bestätigt das Vorliegen von Fumigaclavin $A$.

\begin{tabular}{|c|c|c|c|}
\hline Atom & $\begin{array}{c}\text { Isofumigaclavin A } \\
(\underline{\mathbf{3 0}})\end{array}$ & $\begin{array}{c}\text { Fumigaclavin A } \\
(\underline{\mathbf{3 1}})\end{array}$ & $\underline{\mathbf{x}}$ \\
\hline $\mathrm{C}-2$ & 117.5 & 117.7 & 117.7 \\
\hline $\mathrm{C}-3$ & 111.7 & 111.6 & 111.2 \\
\hline $\mathrm{C}-4$ & 28.2 & 26.8 & 26.6 \\
\hline $\mathrm{C}-5$ & 66.2 & 61.9 & 61.8 \\
\hline $\mathrm{C}-7$ & 62.2 & 57.9 & 57.8 \\
\hline $\mathrm{C}-8$ & 37.7 & 33.2 & 33.0 \\
\hline $\mathrm{C}-9$ & 78.9 & 71.6 & 71.4 \\
\hline $\mathrm{C}-10$ & 45.7 & 39.8 & 39.6 \\
\hline $\mathrm{C}-11$ & 131.4 & 129.9 & 129.7 \\
\hline $\mathrm{C}-12$ & 114.4 & 112.9 & 112.8 \\
\hline $\mathrm{C}-13$ & 123.3 & 123.2 & 123.1 \\
\hline $\mathrm{C}-14$ & 109.0 & 108.3 & 108.4 \\
\hline $\mathrm{C}-15$ & 133.5 & 133.6 & 133.5 \\
\hline $\mathrm{C}-16$ & 126.6 & 126.8 & 126.7 \\
\hline $17-\mathrm{CH}_{3}$ & 15.9 & 16.8 & 16.7 \\
\hline $\mathrm{N}-\mathrm{CH}_{3}$ & 42.7 & 43.6 & 43.5 \\
\hline $\mathrm{C}-18$ & 171.5 & 170.8 & 170.9 \\
\hline $19-\mathrm{CH}_{3}$ & 21.8 & 21.2 & 21.1 \\
\hline
\end{tabular}

Tabelle 3: ${ }^{13} \mathrm{C}$-Verschiebungen in $\mathrm{CDCl}_{3}$ von Isofumigaclavin $\mathrm{A}(\underline{\mathbf{3 0}})$, Fumigaclavin $\mathrm{A}(\underline{\mathbf{3 1}})$ und der aus WDMH35 isolierten Substanz $(\underline{\mathbf{x}})$. 


\section{Fumitremorgin C (르)}

Die mit Anis grün-braun anfärbende Substanz wurde von dem Aspergillus-Stamm sowohl ins Kulturfiltrat als auch ins Myzel ausgeschieden und konnte in einer Ausbeute von mehr als $6 \mathrm{mg} / \mathrm{L}$ isoliert werden. Die chemischen Verschiebungen von $\delta_{\mathrm{C}}=169.4$ und $165.7 \mathrm{im}{ }^{13} \mathrm{C}-\mathrm{NMR}-S p e k t r u m$ lassen die Anwesenheit von zwei Amidbindungen vermuten, was durch eine Schwingungsbande im IR-Spektrum bei $1660 \mathrm{~cm}^{-1}$ bestätigt wurde. Im ${ }^{1} \mathrm{H}$-NMR-Spektrum können im aliphatischen Bereich neben einer Methoxyfunktion $\left(\delta_{H}=3.80\right)$ zwei Methylgruppen identifiziert werden, die im $\mathrm{H}, \mathrm{H}-\mathrm{COSY}$-Spektrum eine Kopplung zu dem Signal bei $\delta_{\mathrm{H}}=4.88$ aufweisen. Neben dieser $1.0 \mathrm{~Hz}$ großen ${ }^{3} \mathrm{~J}$-Kopplung kann man dem Signal des olefinischen Protons eine Aufspaltung zum Dublett $(J=9.5 \mathrm{~Hz})$ entnehmen und die Methingruppe bei $\delta_{\mathrm{H}}=5.97(\mathrm{~d}, J=9.5 \mathrm{~Hz}$ ) als Nachbarn ausmachen. Zwei im Tieffeld befindliche Signale sprechen aufgrund der auftretenden Kopplungskonstanten von $J=8.5 \mathrm{~Hz}$ dafür, dass diese aromatischen Protonen ortho zueinander positioniert sind. Eine weitere kleine Kopplung $(J=c a .1 \mathrm{~Hz}) \mathrm{zu}$ dem Wasserstoffatom bei $\delta_{\mathrm{H}}=6.82$ läßt auf einen 1,2,4-trisubstituierten Aromaten schließen. Aus dem Protonenspektrum können in Kombination mit dem $\mathrm{H}, \mathrm{H}-$ COSY-Experiment zwei weitere Spinsysteme abgeleitet werden. Eine Datendankrecherche in $\underline{\text { Antibase }}^{47}$ (Abbildung 6) ergab drei Treffer.<smiles>COc1ccc2c3c([nH]c2c1)C(C=C(C)C)N1CC3C(=O)N2CCCC2C1=O</smiles>

(므)<smiles>COc1ccc2c(c1)NC(=O)C21CC2C(=O)N3CCCC3C(=O)N2C1C=C(C)C</smiles>

Spirotryprostatin A
Tryprostatin A

(33) 


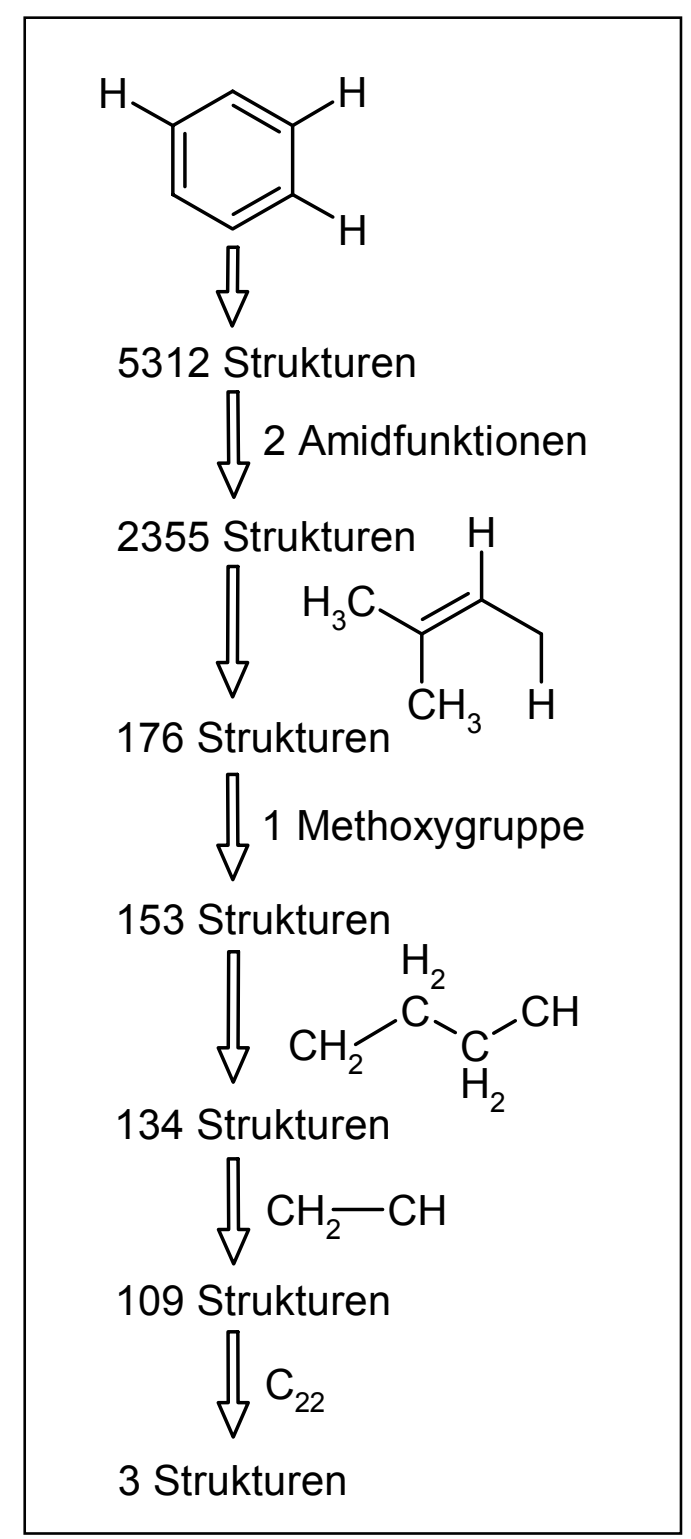

Abbildung 6: Datenbankrecherche für $\underline{32}$ mit Antibase

Im El-Massenspektrum tritt der Peak höchster Masse bei 379 auf, anhand dessen Fumitremorgin C (2) als richtige Lösung ausgewählt wurde. Ein Vergleich der Literaturwerte $^{64}$ mit den ermittelten Daten führte zur Übereinstimmung. $\underline{32}$ inhibiert den Zellzyklus von Säugetierzellen durch Einwirkung auf das Mikrotubulisystem. ${ }^{65}$ 
Zu ungefähr der gleichen Zeit wurde in der Arbeitsgruppe ZEECK ein weiterer Stamm der Gattung Aspergillus fumigatus von P. AlVERMANN bearbeitet. Die in WDMH35 entdeckten Naturstoffe waren auch schon aus diesem isoliert worden. Um weitere unnötige Reisolationen bekannter Pilzmetabolite zu vermeiden, wurden die Fraktionen der Rohproduktsäulen einer HPLC-MS-Analyse unterzogen und nach Massen der Verbindungen gesucht, die von P. ALVERMANN während ihrer Dissertation ${ }^{66}$ gefunden wurden.

\begin{tabular}{|c|c|c|}
\hline $\begin{array}{l}\text { Masse der } \\
\text { isolierten } \\
\text { Substanzen }\end{array}$ & $\begin{array}{l}\text { isolierte Verbindungen aus } \\
\text { Aspergillus fumigatus }\end{array}$ & $\begin{array}{c}\text { HPLC-MS- } \\
\text { Analyse }\end{array}$ \\
\hline \multirow{2}{*}{431} & cis-Pseurotin (29) & \multirow{2}{*}{ positiv } \\
\hline & trans-Pseurotin $(\underline{\mathbf{3 5}})$ & \\
\hline 381 & Tryprostatin A ( $\underline{\mathbf{3 3}})$ & positiv \\
\hline 443 & Fumiquinazolin C ( $\underline{\mathbf{3 6}})$ & positiv \\
\hline 458 & Fumagillin (37) & positiv \\
\hline 511 & Verruculogen ( $\underline{\mathbf{3 8}})$ & positiv \\
\hline 411 & Cyclotryprostatin A ( $\underline{\mathbf{3 9}})$ & positiv \\
\hline 479 & 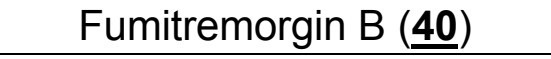 & positiv \\
\hline 393 & 16-Methoxy-spirotryprostatin & negativ \\
\hline 429 & Verruculogen TR-2 & negativ \\
\hline
\end{tabular}

Tabelle 4: Ergebnisse der HPLC-MS-Analyse der WDMH35-Fraktionen im Bezug auf die Molmassen der von P. ALVERMANN isolierten Metabolite.

Die Auswertung der HPLC-MS-Spektren legt nahe, dass es sich bei den Aspergillus-Isolaten um einen ähnlichen, wenn nicht gar gleichen Stamm handelt. Die Massenanalyse gibt einen Hinweis darauf, dass die in Abbildung 7 dargestellten Metabolite ebenfalls vom Stamm WDMH35 gebildet werden. Auf eine weitere Isolierung wurde verzichtet. 


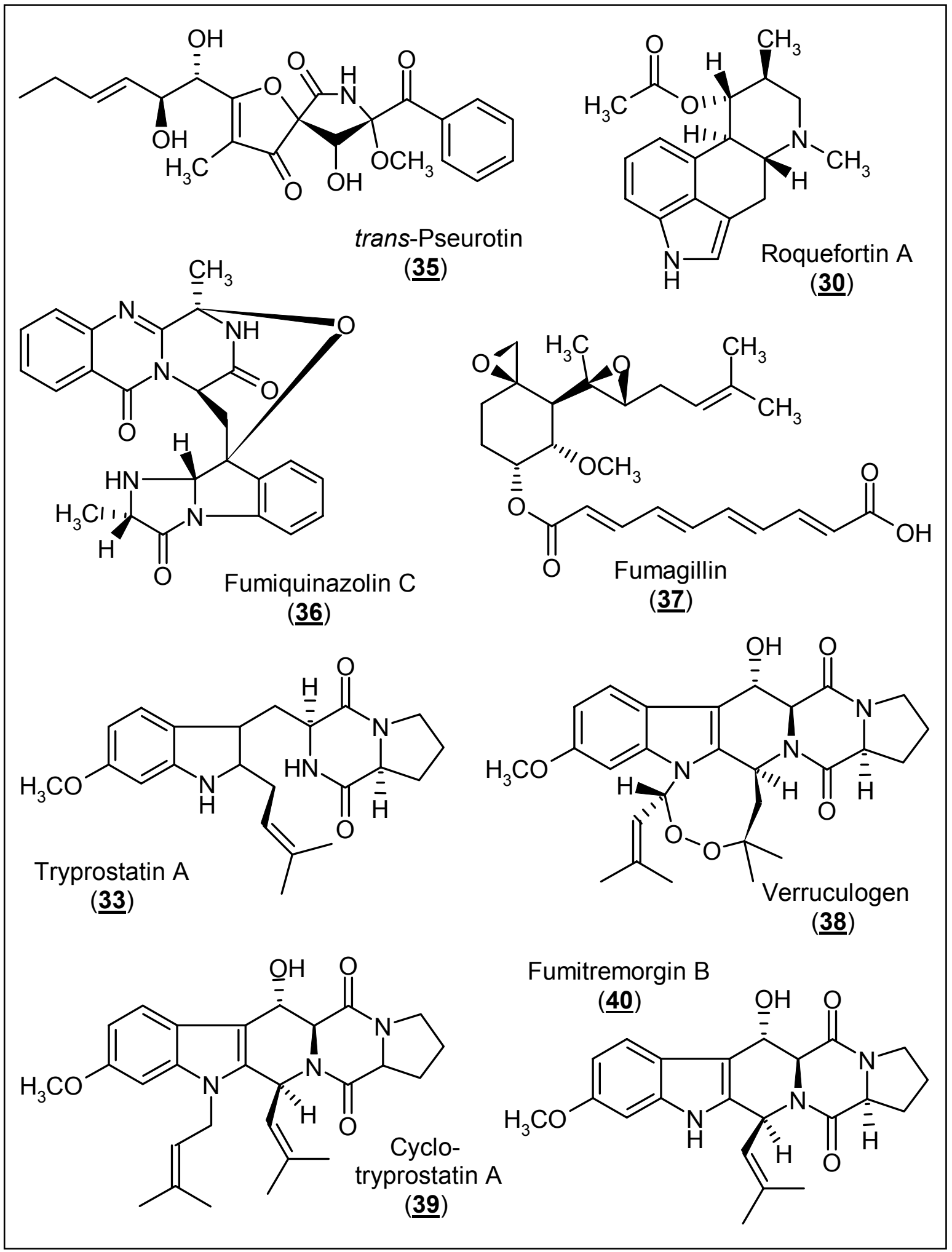

Abbildung 7: Sekundärmetabolite aus Aspergillus fumigatus, die möglicherweise auch von WDMH35 gebildet werden. 


\subsection{WDMHC2}

Das Dünnschichtchromatogramm des Stammes WDMHC2 zeigte abhängig von den Nährmedien eine Vielzahl von Spots, die mit Anis dunkelgrau, mit Orcin bräunlich und mit VS pink anfärbten. Eine Reproduktion im 5 L-Ansatz (NL 1158) erwies sich als weniger erfolgreich, denn es wurde nur ein Hauptmetabolit produziert. Weitere Verbindungen waren nur als Minderkomponenten zu detektieren.

\subsubsection{Isolierung und Charakterisierung von Cyclonerodiol (년}

Der Kulturfiltrat-Extrakt wurde an Kieselgel aufgetrennt. Für die Rohproduktsäule diente ein Dichlormethan/Methanol-Lösungsmittelgemisch mit einem Gradienten von 20:1 nach 4:1 als Eluent. Die weitere Aufreinigung erfolgte isokratisch mit Essigester/Cyclohexan 2:1.

Das ESI-Massenspektrum des farblosen Öles weist im positiven Modus einen schwachen Massenpeak von $263 \mathrm{~g} / \mathrm{mol}$ für $[\mathrm{M}+\mathrm{Na}]^{+}$. Im IR-Spektrum sind neben der charakteristischen $\mathrm{OH}-S t r e c k s c h w i n g u n g$ bei $3430 \mathrm{~cm}^{-1} 1$ weitere Absorptionsbanden bei 1456 und $1379 \mathrm{~cm}^{-1}$ zu sehen, die auf die Anwesenheit von Methylgruppen hindeuten. Aufgrund der fehlenden IR-Frequenzen um $1720 \mathrm{~cm}^{-1}$ können $\mathrm{C}=\mathrm{O}-$ Funktionen als Bestandteil der Struktur ausgeschlossen werden.

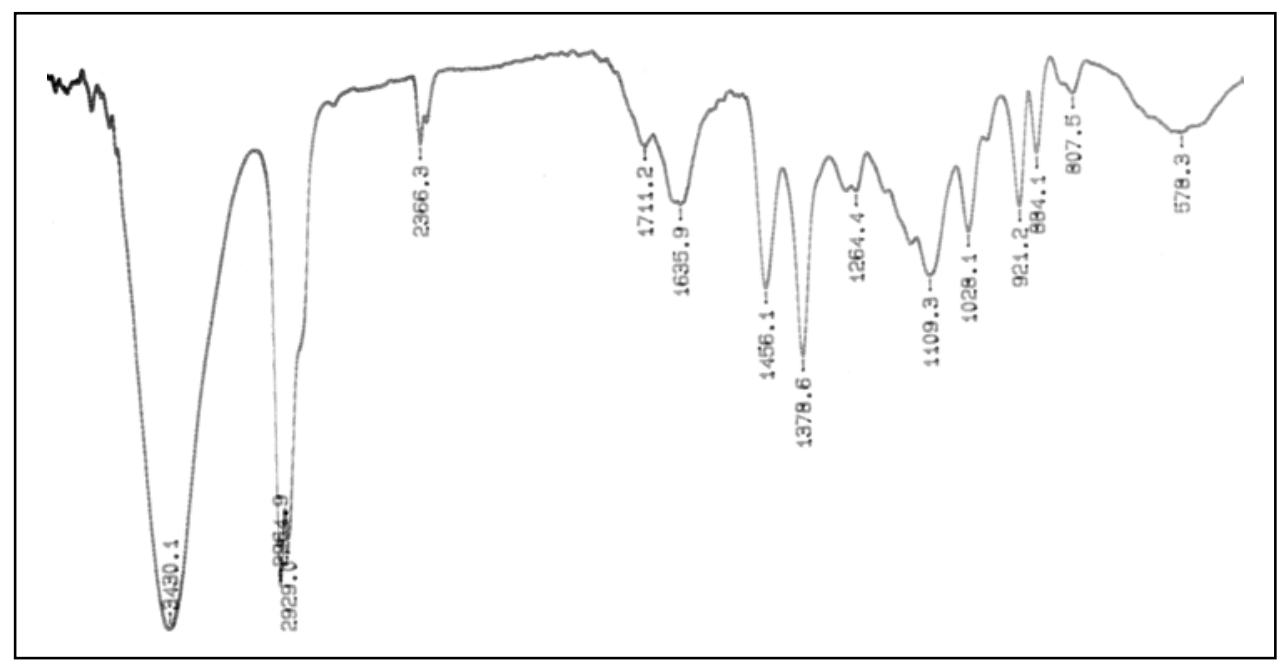

Abbildung 8: IR-Spektrum von (누). 
Das ${ }^{13} \mathrm{C}-N M R-S p e k t r u m$ zeigt fünfzehn Signale. Anhand des APT-NMR-Experimentes erkennt man drei quartäre Kohlenstoffe, ein olefinisches bei $\delta_{C}=130.8$ und zwei an Sauerstoff gebundene C-Atome mit einer Tieffeldverschiebung von 80.3 und 73.9 ppm. Von den drei Methingruppen ist ein C-Atom $\left(\delta_{C}=126.1\right) \mathrm{sp}^{2}-$ hybridisiert, die beiden anderen $\left(\delta_{C}=55.5\right.$ und 45.2) liegen als $\mathrm{sp}^{3}$-Zentrum vor. Des Weiteren sind vier Methylengruppen $\left(\delta_{C}=2 \times 41.7,25.3\right.$ und 23.4) und fünf Methylgruppen bei $\delta_{\mathrm{C}}=26.8,25.8,25.6,17.7$ und $15.7 \mathrm{zu}$ beobachten. Vier der Methylgruppen erscheinen im ${ }^{1} \mathrm{H}-\mathrm{NMR}-\mathrm{Spektrum}$ als Singulett $\left(\delta_{\mathrm{H}}=1.42,1.47\right.$, 1.62 und 1.69), eine weitere $\left(\delta_{\mathrm{H}}=1.41\right)$ koppelt mit $7.0 \mathrm{~Hz}$ zu einer Methingruppe. Tieffeldverschoben finden sich Signale zweier Hydroxylgruppen als breite Singuletts $\left(\delta_{H}=5.00,5.02\right)$ und das eines olefinischen Protons $\left(\delta_{H}=5.31\right.$, tsept, $J=7.0,1.0 \mathrm{~Hz}$ ). Im Bereich von $\delta_{H}=1.75$ bis 2.56 sind die aliphatischen Protonen angesiedelt. Aufgrund vieler Überlagerungen fällt es schwer, die Kopplungspartner anhand von Kopplungskonstanten auszumachen. Durch HSQC- ${ }^{1} J_{(\mathrm{C}, \mathrm{H})}-K_{\mathrm{K}}$ opplungen ließen sich Kohlenstoffatome und daran gebundene Protonen zuordnen. Mit Hilfe eines $\mathrm{H}, \mathrm{H}-\mathrm{COSY}$-Experimentes konnten zwei Spinsysteme aufgestellt werden.

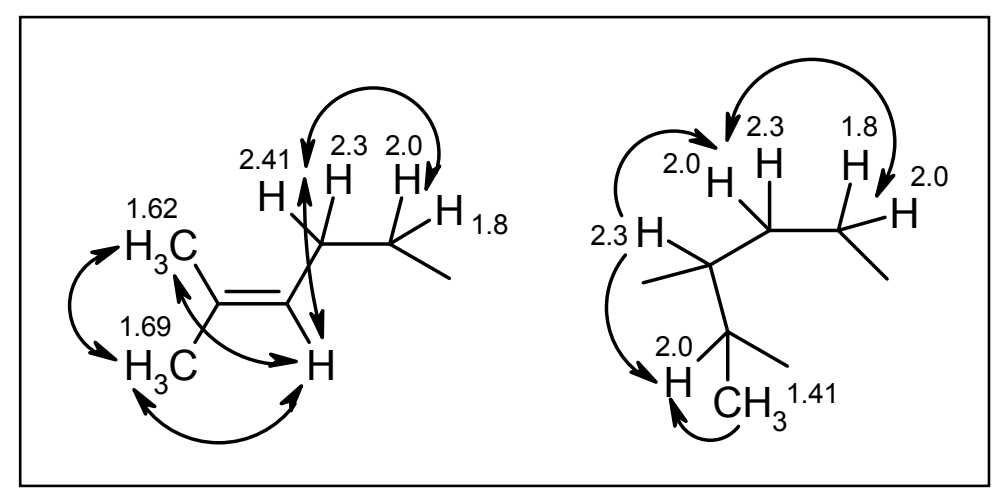

Abbildung 9: ausgewählte $\mathrm{H}, \mathrm{H}-\mathrm{COSY}-K o p p l u n g e n ~ i n ~ \underline{43}$.

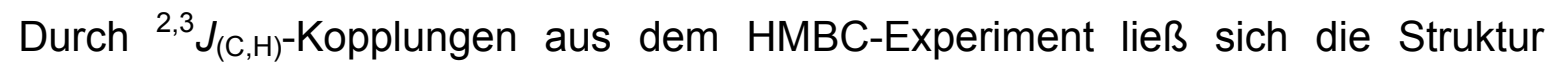
vervollständigen. Ein Datenabgleich in $\underline{\text { Antibase }}^{47}$ ergab, dass es sich bei der isolierten Verbindung $\underline{\mathbf{4 3}}$ um Cyclonerodiol handelt. Der Metabolit wurde erstmals aus Trichothesium roseum isoliert, ${ }^{67}$ später wurde auch von Cyclonerodiol aus Gibberella fujikuroi, ${ }^{68}$ Fusarium cumlorum ${ }^{69}$ und Trichoderma koningi ${ }^{70}$ berichtet. 
Das Sesquiterpendiol wird über den Mevalonat-Stoffwechselweg gebildet. ${ }^{71}$ In einer Biosynthesestudie konnten D.E. CANE et al. zeigen (Abbildung 10), dass zellfreie Enzyme von Gibberella fujikuroi Farnesylpyrophoshat (41) in Nerolidylpyrophosphat (느) umwandeln und über eine CyclonerodiolpyrophosphatZwischenstufe $\underline{43}$ entsteht. $^{72}$

Cyclonerodiol (43) weist weder antifungische noch antibakterielle Aktivität auf, aber inm wurde eine Wachstumsinhibition etiolierter Weizenkeimscheideblätter zugeschrieben. $^{70}$

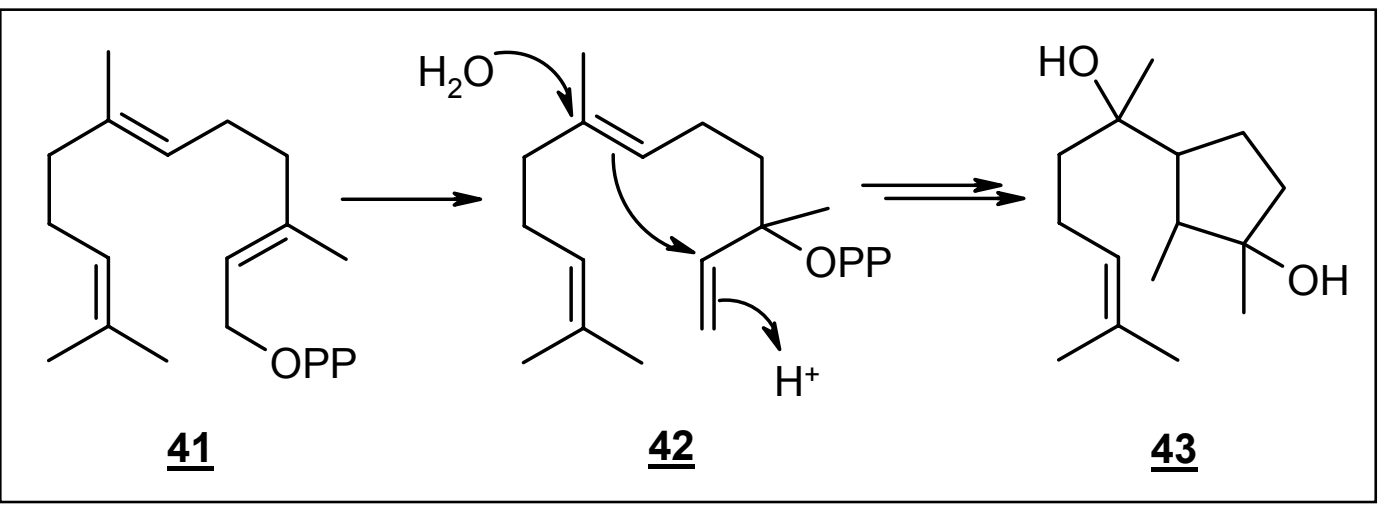

Abbildung 10: Im Verlauf der Biosynthese von $\underline{43}$ erfolgt eine Isomerisierung von Farnesylpyrophoshat (41) in Nerolidylpyrophosphat (42), die Addition von Wasser führt zu Cyclonerodiolpyrophosphat und schließlich zum Cyclonerodiol (프).

\subsection{WDMH24}

In allen Nährmedien, besonders in NL 1158 und G20, war auf den DCs ein UVlöschender Spot zu detektieren, der mit Anis bläulich-braun und mit Ehrlich grau anfärbte. Die durch Orcin hervorgerufene lila Färbung wandelte sich mit der Zeit in eine fluoreszierend wirkende Zone um.

\subsubsection{Isolierung und Charakterisierung von $\underline{44}$}

Der Kulturfiltrat-Extrakt eines 5 L-Ansatzes in Schüttelkolben (NL 1158, $28^{\circ} \mathrm{C}$, 120 spm, 6 Tage) wurde durch säulenchromatographische Trennung an Kieselgel (Dichlormethan/Methanol 99:1 $\rightarrow$ 32:1) fraktioniert und 44 anschließend durch 
Sephadex LH-20 (Aceton) vollständig aufgereinigt. Man erhielt $22.7 \mathrm{mg}$ eines weißen kristallinen Feststoffes.

Eine Masse von $163 \mathrm{~g} / \mathrm{mol}$, ermittelt mit ESI-MS (186 [M+Na] $]^{+}, 349[2 \mathrm{M}+\mathrm{Na}]^{+}$und $\left.162[\mathrm{M}-\mathrm{H}]^{-}\right)$, ließ ein kleines Stickstoff haltiges Molekül erwarten. Die IR-Frequenz bei $v=1666 \mathrm{~cm}^{-1}(\mathrm{C}=\mathrm{O}$ Streckschwingung) deutet ein Amid an. In diesem Bereich (1650-1610 $\mathrm{cm}^{-1}$ ) liegen auch die Banden der $\mathrm{C}=\mathrm{C}$ Streckschwingungen von Olefinen, die Frequenz von $1610 \mathrm{~cm}^{-1}$ spricht für eine konjugierte Doppelbindung. Fehlende Absorbtionen im Bereich von $3000-2900 \mathrm{~cm}^{-1}$ deuten auf die Abwesenheit von Wassertoffatomen hin, die an $\mathrm{sp}^{3}-\mathrm{C}-$-Atome gebunden sind. Das bestätigt auch das ${ }^{1} \mathrm{H}-N M R-S p e k t r u m$ mit Verschiebungen größer $5.5 \mathrm{ppm}$. Die Signale bei $\delta_{\mathrm{H}}=7.20$ und 6.78 reflektieren einen 1,4-disubstituierten Aromaten. Die Resonanz bei $\delta_{H}=6.66(\mathrm{dd}, J=10.5,10.0 \mathrm{~Hz}$ ) kann einem olefinischen Proton zugeordnet werden, das einerseits eine cis-Kopplung zu dem H-Atom mit $\delta_{\mathrm{H}}=5.60$ (d) aufweist und andererseits die Nachbarschaft zu einem Heteroatomgebundenen Proton $\left(\delta_{\mathrm{H}}=9.76\right.$, br d). Neben einer $\mathrm{OH}-F u n k t i o n a l i t a ̈ t ~(b r s ~ b e i$ $\left.\delta_{H}=9.45\right)$ findet man ein weiteres Singulett bei 8.12 ppm, welches erst mit Hilfe des APT-NMR-Spektrums ( $\delta_{\mathrm{C}}=159.9$, Signal nach oben) als Aldehyd-H erkannt wurde. Durch die zwei quartären Kohlenstoffatome bei $\delta_{C}=156.2$ (phenolisch) und 126.1 (aromatisch) wird die Struktur vervollständigt. Ein Strukturvergleich in ${\underline{\text { Antibase }^{47}}}^{47}$ dokumentiert die Isolierung von $\mathrm{N}-(2-$<smiles>O=CN/C=C\c1ccc(O)cc1</smiles>

$\underline{44}$ cis(4-Hydroxyphenyl)ethenyl)-formamid (44), die im Artikel als WF 5239 bezeichnet wurde. WF 5239 aus Aspergillus fumigatus werden antithrombocytische Eigenschaften nachgesagt. ${ }^{73}$ Wie das Substrat die Inhibierung die Thrombocyten-Aggregation bewirkt, ist nicht geklärt. Es wird aber angenommen, dass der Wirkmechanismus wie beim Aspirin über eine Inhibierung der Cyclooxygenase verläuft. 


\subsection{WDMH51}

Die Kulturen der Aspergillus-Spezies WDMH51 wiesen in den verschiedenen Nährlösungen ein unterschiedliches Aussehen auf. Die optischen Eindrücke und die $\mathrm{pH}$-Kontrolle der 4-tägigen Kulturen sind in nachfolgender Tabelle 5 dargestellt.

\begin{tabular}{|c|c|c|c|}
\hline NL & Kulturfiltrat & $\mathrm{pH}-$ Wert & Myzel \\
\hline 1158 & beige-braun & $4-5$ & beige-braun, z.T. grün \\
\hline E2 & rotbraun & 7 & rötlich-beige \\
\hline G20 & rotbraun & 7 & rötlich-beige, z.T. grün \\
\hline 1405 & rötlich-beige & 8 & rötlich-beige \\
\hline 1549 & gelb-beige & $5-6$ & gelb-beige \\
\hline M.B. & beige & 9 & beige \\
\hline
\end{tabular}

Tabelle 5: Aussehen und pH-Wert der Kulturlösungen von WDMH51 nach vier Tagen.

Die angefärbten Dünnschichtchromatogramme konnten diesen Eindruck nur zum Teil bestätigen. Der UV-löschende und fluoreszierende Hauptmetabolit mit gelber Eigenfarbe lag in allen Kulturen vor. Die Unterschiede der Nährmedienvariation zeigten sich in der Bildung von Nebenkomponenten.

\subsubsection{Isolierung und Charakterisierung der Metabolite}

Für ein scale up im 5 L-Maßstab schien das Nährmedium G20 am geeignetsten. Die Fermentation über sechs Tagen wurde bei $28^{\circ} \mathrm{C}$ und $120 \mathrm{spm}$ in $1 \mathrm{~L}$ Schüttelkolben ohne Schikanen (170 mL NL) durchgeführt. Nach Trennung von Kulturfiltrat und Myzel beschränkte man sich auf die Aufreinigung des MyzelExtraktes. Die genaue Vorgehensweise ist in Abbildung 11 aufgeführt. 


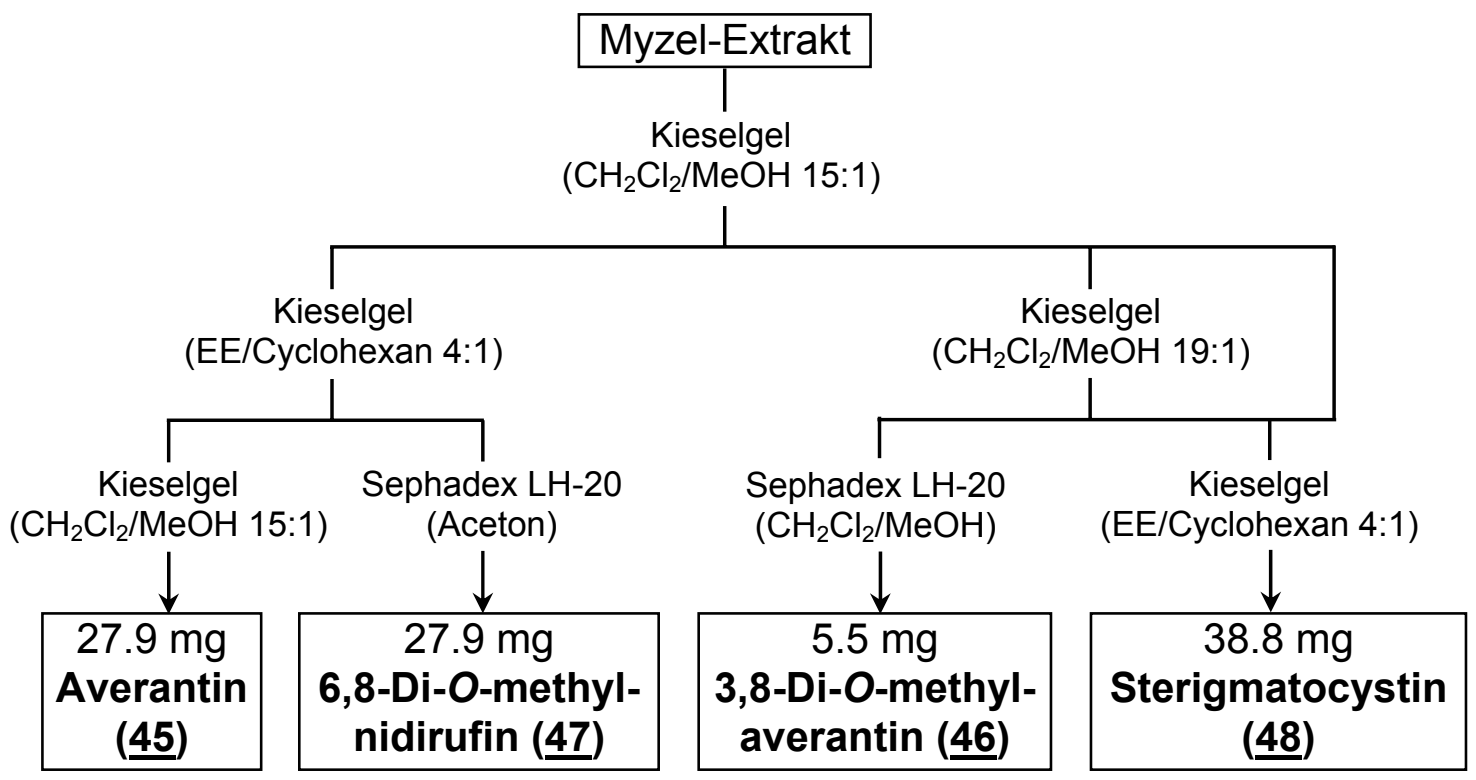

Abbildung 11: Aufarbeitungsschema von Kulturfiltrat- und Myzel-Extrakt (5 L-Ansatz) des Aspergillus-Stammes WDMH35.

\section{Averantin (노)}

Aus einem ESI-Massenspektrum der orangefarbenen Verbindung kann man eine Molmasse von $372 \mathrm{~g} / \mathrm{mol}$ ableiten. Die barthochrome Verschiebung des Absorptionsmaximums im UV-Spektrum von 293 auf $320 \mathrm{~nm}$ beim Wechsel von neutraler zu basisch methanolischer Lösung deutet auf die Anwesenheit einer phenolischen Hydroxygruppe hin. Im IR-Spektrum ist neben der charakteristischen $\mathrm{OH}$ Streckschwingung bei $3400 \mathrm{~cm}^{-1}$ eine Bande bei $1620 \mathrm{~cm}^{-1} \mathrm{zu}$ sehen. Diese entsteht durch die Streckschwingung zum einen von $\mathrm{C}=\mathrm{C}-$ Bindungen, zum anderen von enolisierten $\beta$-Dicarbonylverbindungen. Dem Protonenspektrum $\left(D_{6}\right.$-Aceton) lassen sich Signale zweier chelatisierter $\mathrm{OH}-G$ ruppen $\left(\delta_{H}=13.73\right.$, 12.17) entnehmen. Ferner erkennt man die Resonanzen dreier aromatischer Protonen, einer Sauerstoff-benachbarten $\mathrm{CH}$-Gruppe und das Spinsystem einer Alkyl-Kette. Im ${ }^{13}$ C-NMR-Spektrum finden sich Signale für 20 Kohlenstoffatome, darunter zwölf eines Aromaten (vier davon an Sauerstoff gebunden), zwei Carbonylgruppen bei $\delta_{C}=190.5$ und 181.7, einer Methylgruppe, vier Methylengruppen sowie ein protonentragendes Sauerstoff-substituiertes Kohlenstoffatom. 
Aus dem H,H-COSY lässt sich die Konnektivität der Alkyl-Kette ableiten. Durch ein HSQC- und HMBC-Experiment gelingt die vollständige Verknüpfung der Fragmente. Die Struktur war in $\underline{\text { Antibase }}^{47}$ als Averantin (45) ${ }^{74,75}$ eingetragen.
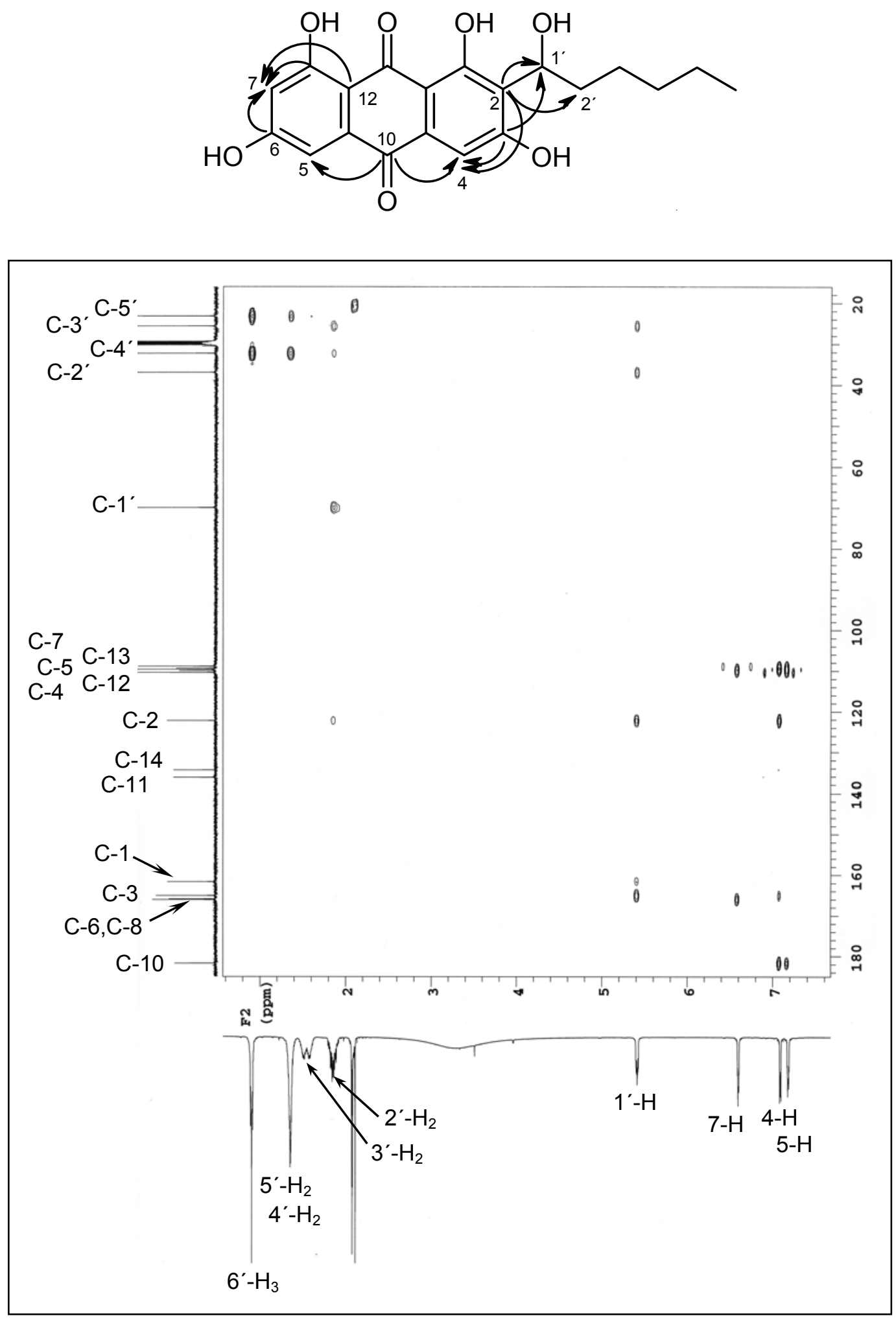

Abbildung 12: HMBC-Spektrum von $\underline{45}$ sowie daraus abgeleitete Verknüpfungen. 


\section{3,6-Di-O-methylaverantin (ㅌ)}

Der zweite orangefarbene Metabolit aus WDMH51 ist unpolarer als $\underline{45}$ und färbt mit Anis ebenfalls orange an. Die Lage des Absorptionsmaximums im UV-Spektrum bei $285 \mathrm{~nm}$, das in basischer Lösung barthochrom zu $310 \mathrm{~nm}$ verschoben wird, deutet hier ebenfalls auf die Anwesenheit eines Phenols hin.

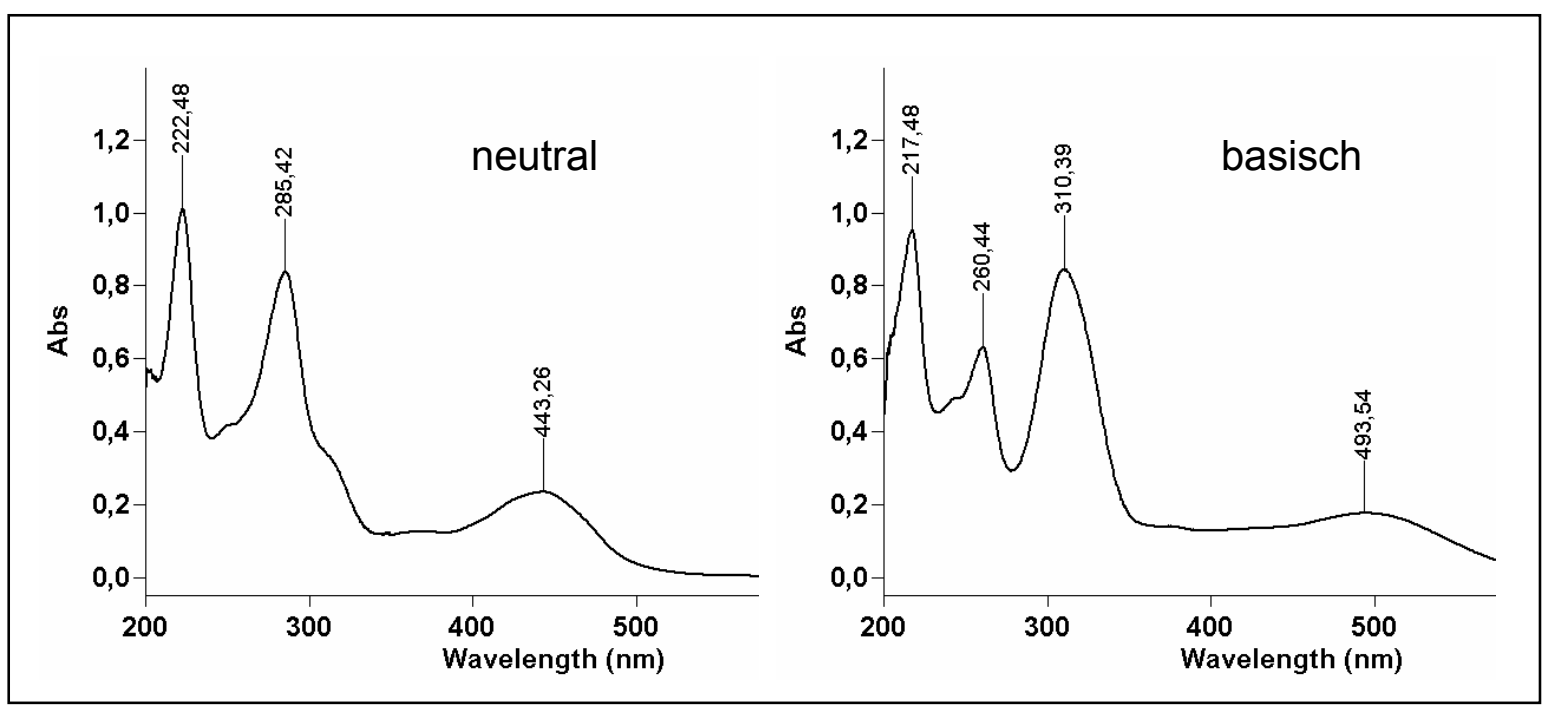

Abbildung 13: UV-Spektrum von $\underline{46}$ in methanolischer Lösung; links: neutral, rechts: basisch.

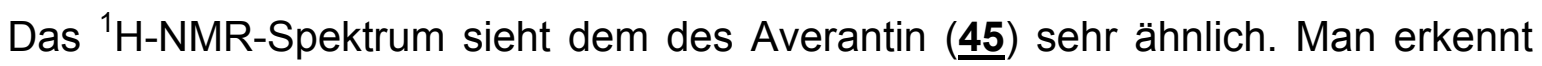
die drei aromatischen Protonen, die Signale der Alkyl-Kette im Bereich von $\delta_{\mathrm{H}}=0.87$ bis 1.90 mit der sauerstoffsubstituierten Methingruppe bei $\delta_{\mathrm{H}}=5.43$ und zwei Singuletts der Hydroxygruppen, von denen eine hochfeldverschoben $\left(\delta_{H}=9.66\right)$ vorliegt. Das kann zum einen mit der Chelatisierung zusammenhängen, zum anderen mit dem Lösungsmittelwechsel von $\mathrm{D}_{6}$-Aceton zu $\mathrm{CDCl}_{3}$. Außerdem tauchen zwei Singulett-Signale auf, deren Intensität und Verschiebung $\left(\delta_{\mathrm{H}}=4.00\right.$ und 4.02) zwei Methoxygruppen kennzeichnen. Im ${ }^{13} \mathrm{C}-\mathrm{NMR}-S p e k t r u m$ treten die zusätzlichen Resonanzen bei $\delta_{C}=56.0$ und 56.5 auf.

Durch ESI-Massenspektrometrie wurde eine Masse von $400 \mathrm{~g} / \mathrm{mol}$ ermittelt, die die Addition zweier Methylgruppen an das Polyhydroxyanthrachinonsystem bestätigt. Über ein HMBC-Experiment konnte die Lage der Methoxy-Funktionalitäten an C-3 und C-6 bestimmt werden. 
<smiles>CCCCC[C](O)c1c(OC)cc2c(c1O)C(=O)c1c(O)cc(OC)cc1C2=O</smiles>

$\underline{46}$

3,6-Di-O-methylaverantin ist als Naturstoff bisher nicht beschrieben. Eine Datenbankrecherche in SciFinder ${ }^{76}$ Antibase $^{47}$ und Chapman \& Hall ${ }^{77}$ zeigte nur Einträge von Averantin-6-methylether ${ }^{75}$, Averantin-1'-methylether ${ }^{78}$ und 1,8-Di-Omethylaverantin ${ }^{79}$ an.

\section{6,8-Di-O-methylnidurufin (47)}

Für den orange-gelben Farbstoff lässt sich durch ESI-Massenspektrometrie eine Molmasse von $412 \mathrm{~g} / \mathrm{mol}$ bestimmen, die sowohl im negativen als auch im positiven Modus bestätigt wird. Im ${ }^{1} \mathrm{H}-\mathrm{NMR}$-Spektrum erkennt man Ähnlichkeit zu der zuvor beschriebenen Substanz.

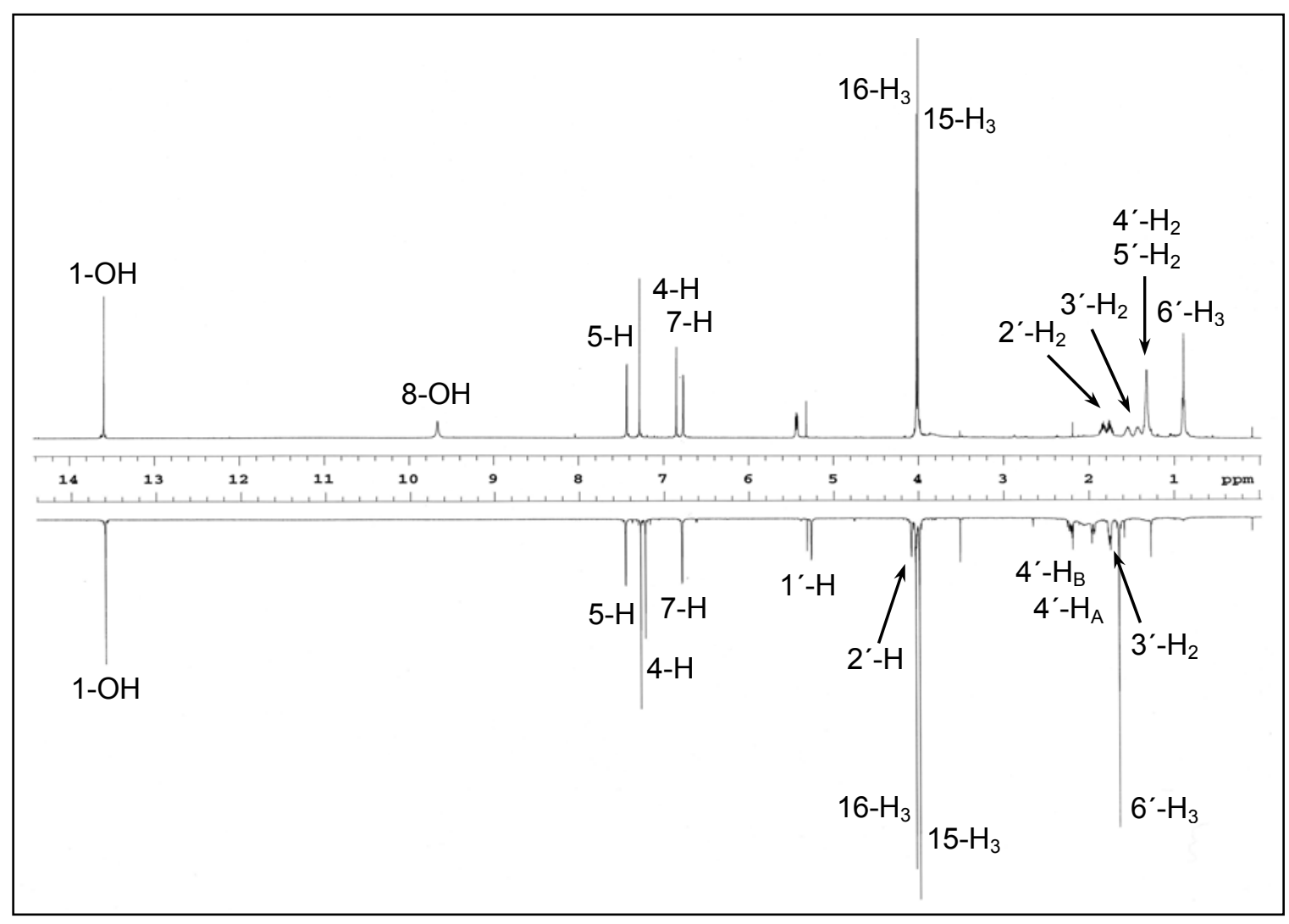

Abbildung 14: ${ }^{1} \mathrm{H}-\mathrm{NMR}-S p e k t r e n$ von $\underline{46}$ (oben) und $\underline{\mathbf{4 7}}$ (unten); $500 \mathrm{MHz}, \mathrm{CDCl}_{3}$. 
Neben dem Verlust einer Hydroxygruppe und zweier Methylengruppen erscheint eine tieffeldverschobene Methingruppe bei $\delta_{\mathrm{H}}=4.10$, für die eine Nachbarschaft zu Sauerstoff angenommen werden kann. Das Proton ist einem Spinsystem zugehörig, welches eine aliphatische Kette von zwei heteroatomgebundenen Methingruppen und zwei Methylengruppen bildet. Das ${ }^{13} \mathrm{C}-\mathrm{NMR}-S p e k t r u m$ lässt wiederum eine Polyhydroxyanthrachinonstruktur mit zwei Methoxygruppen und einer Alkylseitenkette erwarten. Besonders auffällig ist das Signal eines quartären Kohlenstoffatoms, deren chemische Verschiebung von 100.8 ppm das Vorhandensein eines Ketals impliziert.

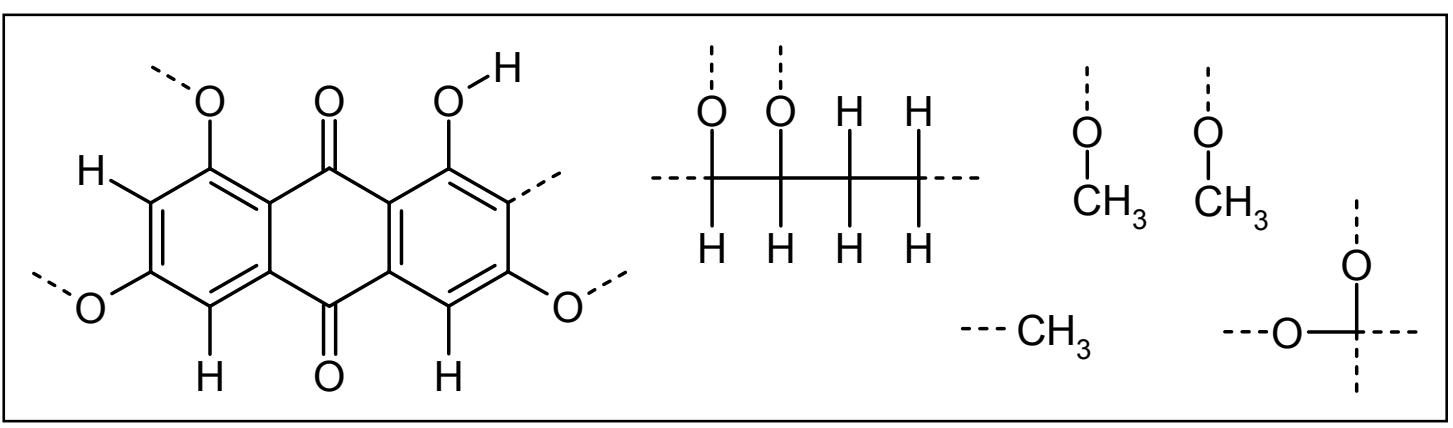

Abbildung 15: Aus den NMR-Spektren abgeleitete Fragmente für $\underline{47}$.

Aus der abgeleiteten Summenformel $\mathrm{C}_{22} \mathrm{H}_{20} \mathrm{O}_{8}$ errechnet sich das Vorliegen von dreizehn Doppelbindungsäquivalenten, von denen elf durch die AnthrachinonStruktur abgedeckt sind. Die Seitenkette muss in diesem Fall durch zwei Ringschlüsse verknüpft sein. Eine Datenbankrecherche in $\underline{\text { Antibase }}^{47}$ mit den bislang ermittelten Strukturelementen (Abbildung 15) führte zu 6,8-Di-O-methylnidurufin $(\underline{47})^{80}$<smiles>[3H]C12CCC(O)[C](O1)c1c(cc3c(c1O)C(=O)c1c(OC)cc(OC)cc1C3=O)O2</smiles> 


\section{Sterigmatocystin (표)}

Der Hauptmetabolit des 5 L-Ansatzes wurde in Form eines hellgelben Feststoffes erhalten. Aufgrund des UV-Spektrums und seines Anfärbeverhaltens mit den verschiedenen Sprühreagenzien kann im Vergleich zu den bisher beschriebenen Metaboliten eine Anthrachinonstruktur ausgeschlossen werden. Dem ${ }^{13} \mathrm{C}-\mathrm{NMR}$ Spektrum sind Signale von 14 Kohlenstoffatomen im $\mathrm{sp}^{2}$-Zentren-Bereich $\mathrm{zu}$ entnehmen, darüber hinaus eine Carbonylfunktion bei $\delta_{C}=181.2$, eine Methoxygruppe, ein protontragendes C-Atom mit einer Verschiebung von 90.4 ppm und eine aliphatische $\mathrm{CH}$-Gruppe. Im Protonenspektrum lassen sich das Spinsystem eines 1,2,3-trisubstituierten Aromaten und eine Kette von vier Methingruppen ausmachen, ferner die drei Singuletts einer chelatisierenden Hydroxygruppe bei $\delta_{H}=13.22$, einer Methin- und einer Methoxygruppe $\left(\delta_{H}=6.41,3.98\right)$.

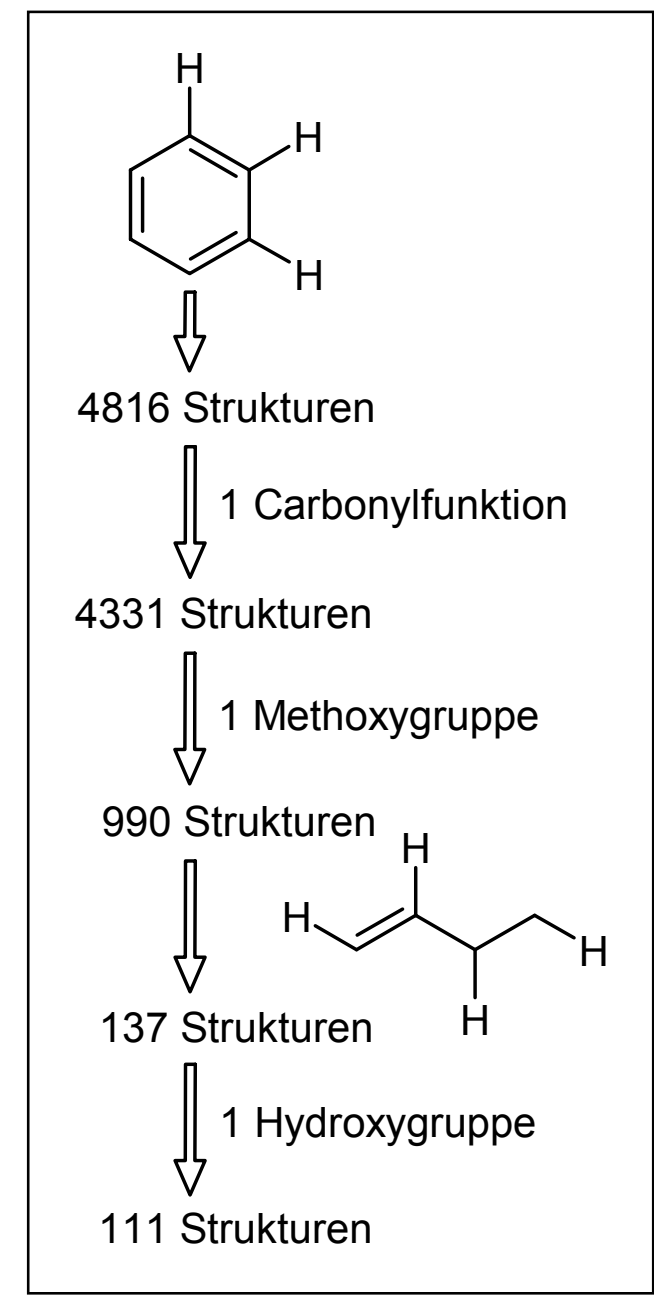

\begin{abstract}
Eine in Antibase $^{47}$ durchgeführte Struktursuche ergab 111 Einträge. Mit Hilfe der Masse von $324 \mathrm{~g} / \mathrm{mol}$, ermittelt durch ESI-MS, konnten diese auf eine reduzierte werden, als Lösung ergab sich Sterigmatocystin (표). ${ }^{54}$
\end{abstract}

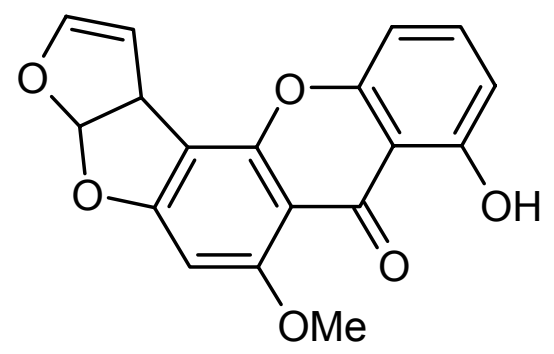

$\underline{48}$
Abbildung 16:

Suchstrategie in Antibase für $\underline{48}$. 
<smiles>CCCCCC(=O)CC(=O)CC(=O)CC(=O)CC(=O)CC(=O)CC(=O)CC(=O)OCC</smiles><smiles>CCCCCC(=O)c1c(O)cc2c(c1O)C(=O)c1c(O)cc(O)cc1C2=O</smiles>

$(\underline{45})$<smiles>CC12CCCC(O1)c1c(cc3c(c1O)C(=O)c1c(O)cc(O)cc1C3=O)O2</smiles><smiles>CC(=O)OCCC1c2c(cc3c(c2O)C(=O)c2c(O)cc(O)cc2C3=O)OC1O</smiles>
Halbacetal Acetat<smiles>O=C1c2cc(O)cc(O)c2C(=O)c2c1cc1c(c2O)C2CCOC2O1</smiles><smiles>CC(C)CCCO</smiles><smiles>O=C1c2cc(O)cc(O)c2C(=O)c2c1cc1c(c2O)C2C=COC2O1</smiles><smiles>CCCCCCCC</smiles><smiles>COc1cc2c(c3oc4cccc(O)c4c(=O)c13)C1C=COC1O2</smiles><smiles>COc1cc2c(c3oc(=O)c4c(c13)CCC4=O)C1C=COC1O2</smiles>

$(\underline{48})$<smiles>COc1cccc2oc3c4c(cc(OC)c3c(=O)c12)OC1OC=CC41</smiles>

Abbildung 17: Die Biosynthese des Aflatoxins. 
Alle aus diesem Stamm isolierten Metabolite stellen Biosynthesestufen des Aflatoxin $B_{1}(\underline{49})$ dar. $\underline{49}$ zählt zu den Mykotoxinen ${ }^{81}$ und wurde erstmals 1960 auffällig, als es als Ursache für das Massensterben junger Puten in England ${ }^{82}$ entdeckt wurde. ${ }^{83}$ Aflatoxine, von Aspergillus flavus und Aspergillus parasiticus gebildet, und auch einige Vorstufen wirken karzinogen, teratogen, hepatotoxisch und immunsuppressiv. ${ }^{84,85}$ Von einer Weiterbearbeitung des Stammes WDMH51 wurde Abstand genommen. 


\section{Stamm WDMH28}

Das Pilzisolat WDMH28 wurde der Abteilung ZEECK mit der Einordnung „steriles Mycel" überlassen. Auch nach einer ITS1- bzw. 18S-rDNA-Sequenzierung, durchgeführt von Dipl.-Biologe M. HAIN an der Universität Oldenburg, konnte das Isolat anhand der Sequenzdaten durch Datenbankanalyse nicht zugeordnet werden.

Der Stamm WDMH28 fiel im Chemischen Screening dadurch auf, dass er in den Medien 1158, E2, G20 und 1549 zwei bis drei UV-löschende Hauptmetabolite bildete, die mit Anis blaugrau anfärbten. Diese waren sowohl im Kulturfiltrat als auch im Myzel nachzuweisen. Es war außerdem zu erkennen, dass die Substanzen schon nach einer Fermentationsdauer von vier Tagen vorlagen, aber bei einer Ernte nach sechs Tagen die Substanzenge deutlich erhöht war.

\subsection{Fermentation und Isolierung der Metabolite}

WDMH28 wurde bei $28^{\circ} \mathrm{C}$ und $120 \mathrm{spm}$ in $1 \mathrm{~L}-$ Schüttelkolben ohne Schikanen, befüllt mit $170 \mathrm{~mL}$ der Nährlösung 1158, über sechs Tage fermentiert. Die Aufarbeitung der Kulturbrühe ( $5 \mathrm{~L}$ ) erfolgte nach Abbildung 4, wobei der Myzel-Extrakt verworfen wurde. Der Kulturfiltrat-Extrakt wurde einer Säulenchromatographie an Kieselgel (Dichlormethan/Methanol 20:1 $\rightarrow$ 4:1) unterzogen und in 10 Fraktionen (F1-10) unterteilt.

F6 enthielt eine mit Anis rotbraun anfärbende Verbindung $\underline{\mathbf{5 0}}$, die durch Säulenchromatograhie an Kieselgel (Essigester/Methanol 19:1 und $\mathrm{CH}_{2} \mathrm{Cl}_{2} / \mathrm{MeOH}$ 20:1) zu einer Menge von $14.9 \mathrm{mg}$ aufgereinigt wurde.

Durch mehrfache Trennschritte an Kieselgel (Cyclohexan/Essigester/Methanol 5:10:1) und Sephadex LH-20 $\left(\mathrm{CH}_{2} \mathrm{Cl}_{2} / \mathrm{MeOH} 1: 1\right.$ und Aceton) ließen sich aus der Rohproduktfraktion F3 eine Reinsubstanz $\underline{61}(5.3 \mathrm{mg})$ und eine Mischfraktion erhalten. Die im Verhältnis 3:2 vorliegenden Metabolite konnten nach analytischer Optimierung (HPLC 1, Säule 2, Programm 2) über die präparative HPLC (HPLC 3 , Säule 5, Programm 7) zur Reinheit gebracht werden. Von den farblosen Verbindungen erhielt man $8.1 \mathrm{mg} \underline{\mathbf{5 1}}$ und $6.0 \mathrm{mg} \underline{\mathbf{5 2}}$.

Weitere Aufreinigung von F4 an Sephadex $\mathrm{LH}-20\left(\mathrm{CH}_{2} \mathrm{Cl}_{2} / \mathrm{MeOH}\right.$ 1:1) und Kieselgel (Essigester/Cyclohexan 4:1) ergab den Hauptmetaboliten, der beim Anfärben des Dünnschichtchromatogrammes mit Anis noch nicht einheitlich erschien. Eine 
analytische Kontrolle mit der HPLC (HPLC 1, Säule 1, Programm 1) bestätigte den Eindruck. Deshalb wurde an der analytischen HPLC ein Programm optimiert, mit dem es möglich war, die zwei Metabolite zu trennen (HPLC 2, Säule 3, Programm 4, 1). Etwa 2/3 des Gemisches (von $291 \mathrm{mg}$ ) wurden auf die präparative HPLC (HPLC 3, Säule 4, Programm 5) aufgetragen und 128.3 mg $\underline{\mathbf{5 6}}$ und 35.6 mg $\underline{60}$ als farbloses Öl erhalten.

\subsection{Charakterisierung der Metabolite}

\section{$\alpha$-Acetylorcinol (무)}

Die Verbindung liegt als farbloses Öl vor und färbt mit Anis orange-braun an. Eine El-MS-Analyse lieferte eine Molmasse von $166 \mathrm{~g} / \mathrm{mol}$. Im ${ }^{1} \mathrm{H}-\mathrm{NMR}-S p e k t r u m$ sind drei Signale erkennbar, die Singuletts einer Methyl- und einer Methylengruppe bei $\delta_{\mathrm{H}}=2.10$ und 3.52 , sowie drei aromatische Protonen bei $\delta_{\mathrm{H}}=6.15$. Das ${ }^{13} \mathrm{C}-\mathrm{NMR}$ Spektrum offenbart zusätzlich vier quartäre Kohlenstoffatome: zwei aromatische C-Atome bei $\delta_{C}=137.4$, einen tieffeldverschobenen Kern bei $\delta_{C}=159.8$, sowie das für eine Keto-Funktion typische Signal bei $\delta_{C}=209.7$. Mit diesen Informationen wurde eine Datenbankrecherche in $\underline{\text { Antibase }}^{47}$ durchgeführt. Durch Vergleich mit Literaturdaten ${ }^{86}$ konnte $\underline{\mathbf{5 0}}$ als $\alpha$-Acetylorcinol identifiziert werden.

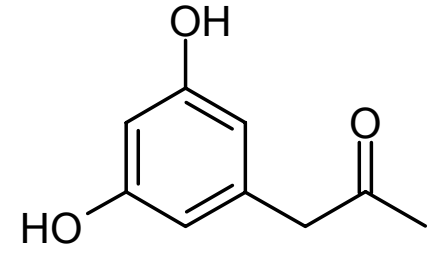

$\underline{50}$

\section{Isoscleron ( $\underline{51})$}

Das farblose Öl ist mit einem $\mathrm{R}_{\mathrm{f}}$-Wert um $0.5\left(\mathrm{CHCl}_{3} / \mathrm{MeOH}\right.$ 9:1) den mittelpolaren Substanzen zuzuordnen und färbt mit Anis blau an. Im El-Massenspektrum ist das positive lon höchster Masse bei $\mathrm{m} / \mathrm{z}=178 \mathrm{zu}$ finden, was einer Summenformel von $\mathrm{C}_{10} \mathrm{H}_{10} \mathrm{O}_{3}$ entspricht.

Im ${ }^{1} \mathrm{H}-\mathrm{NMR}-S p e k t r u m$ erkennt man neun Signale. Die Signale der Methylengruppen bei $\delta_{H}=2.14 / 2.29$ und 2.60/2.95 sowie der Methingruppe bei $\delta_{H}=4.87$ bilden die Gruppe der aliphatischen Kohlenwasserstoffe. Mit Hilfe der Kopplungskonstanten konnten die Konnektivitäten dieser koppelnden Protonen bestimmt werden. Des Weiteren treten neben einem $\mathrm{OH}$-Signal bei $\delta_{\mathrm{H}}=12.37$ die Resonan- 
zen dreier aromatischer Protonen $\left(\delta_{H}=6.87,6.98\right.$ und 7.45) auf. Das Kopplungsmuster und Kopplungskonstanten um $8 \mathrm{~Hz}$ beweisen, dass die H-Atome nebeneinander angesiedelt sind. Aus dem ${ }^{13} \mathrm{C}-\mathrm{NMR}$-Spektrum gehen neben den zwei Methylengruppen und den vier Methingruppen vier quartäre Kohlenstoff-Atome hervor. Außer einer Carbonylfunktion $\left(\delta_{C}=204.3\right)$ und einem phenolischen $C$ bei $\delta_{\mathrm{C}}=162.6$ sind zwei $\mathrm{sp}^{2}$-Zentren detektierbar.

Eine Datenbankrecherche in $\underline{\text { Antibase }}^{47}$ zeigte einen Treffer. Der anschließende Vergleich mit Literaturdaten ${ }^{87}$ führte zur Übereinstimmung mit Isoscleron ( $\left.\underline{\mathbf{5 1}}\right)$. Isoscleron ist ein typischer Pilzmetabolit mit phytotoxischer und antifungischer Aktivität und wurde erstmals aus der Gattung Scytalidum als 4,8-Dihydroxytetralon isoliert. $^{88}$ Von Stereoisomeren wurde nicht nur aus Pilzstämmen berichtet, wie z.B. (+)-Isoscleron aus Sclerotinia sclerotiorum, ${ }^{89}$ sondern auch aus der Rinde eines Walnussbaumes Juglans regia als (-)-Regiolon. ${ }^{90}$ Mit Hilfe des CD-Spektrums kann anhand des positiven bzw. negativen Cotton-Effektes bei 213 und 259 auf (+)-<smiles>O=C1CC[C@H](O)c2cccc(O)c21</smilesIsoscleron ( $\underline{\mathbf{5 1}})$ geschlossen werden.

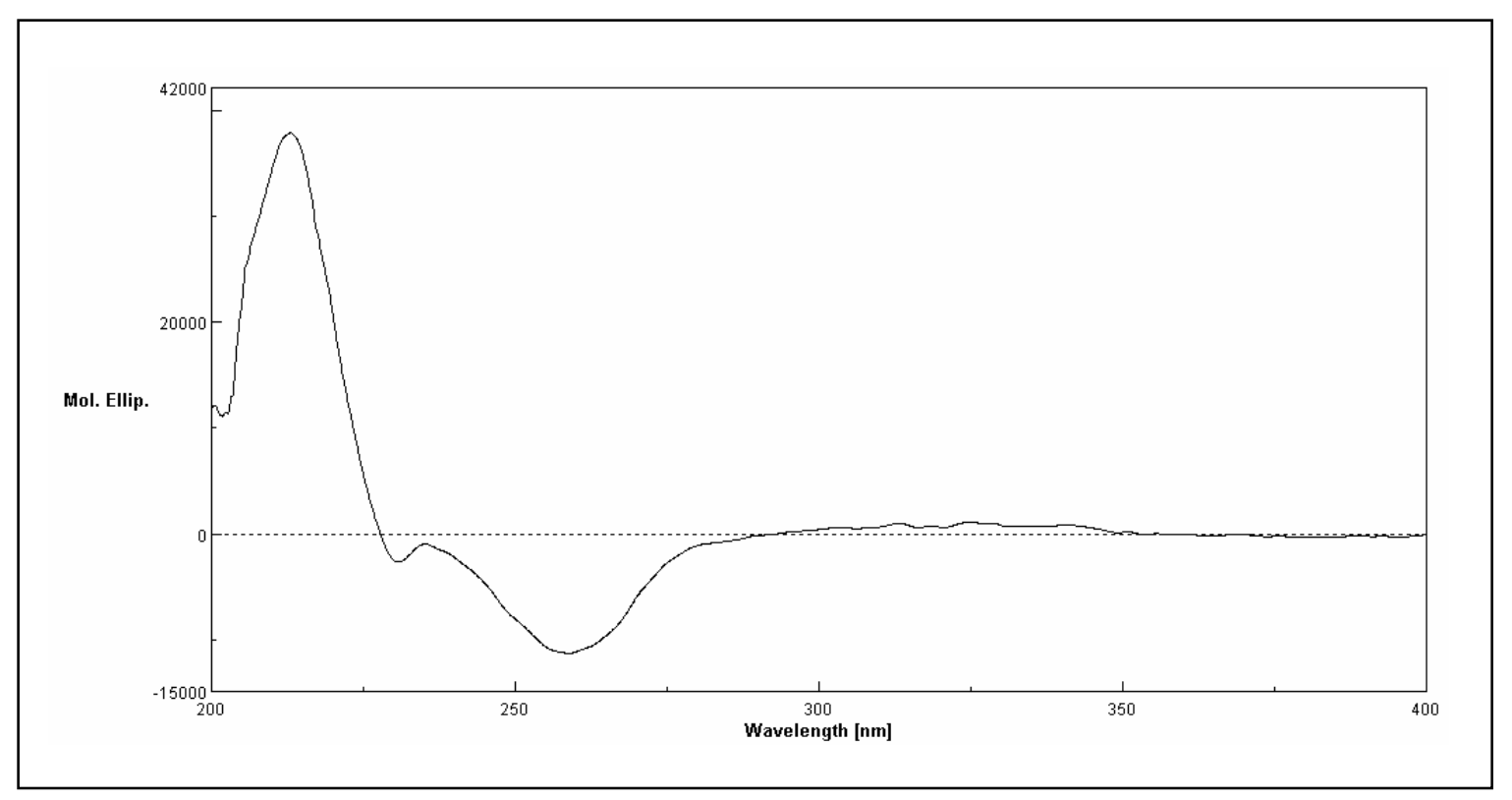

Abbildung 18: CD-Spektrum von Isoscleron (ㅌ1). 


\section{6-Methoxyisoscleron ( $\underline{52})$}

$\underline{52}$ färbt mit Anis violett an und hat laut El-Massenspektrometrie ein Gewicht von $208 \mathrm{~g} / \mathrm{mol}$, was einer Summenformel von $\mathrm{C}_{11} \mathrm{H}_{12} \mathrm{O}_{4}$ gleichkommt.

Die NMR-Spektren sind denen des Isoscleron (1) sehr ähnlich. Im ${ }^{1} \mathrm{H}-\mathrm{NMR}$ Spektrum ist ein aromatisches Wasserstoff-Atom weniger zu erkennen. Die zwei anderen Aromaten-Protonen weisen mit einer Kopplungskonstanten von $2.5 \mathrm{~Hz}$ eine meta-Stellung zueinander auf. Zusätzlich taucht ein Signal mit der Intentität von drei Protonen auf. Die Verschiebung von $\delta_{H}=3.82$ läßt auf eine Methoxygruppe schließen. Es scheint sich um ein an 6-Position O-methyliertes Derivat des Isoscleron zu handeln.<smiles>COc1cc(O)c2c(c1)[C@H](O)CCC2=O</smiles>

Das ${ }^{13} \mathrm{C}-N M R-S p e k t r u m$ bestätigt diese Vermutung. Das Kohlenstoff-Atom der Methingruppe ist durch ein quartäres Sauerstoff-gebundenes $C$ bei $\delta_{C}=164.1$ ersetzt. Außerdem wird das Signal einer Methoxygruppe gefunden $\left(\delta_{C}=56.0\right)$.

$\underline{\mathbf{5 2}}$ weist wie (+)-Isoscleron (1ㅡ) einen positiven Drehwert auf und ist durch einen positiven bzw. negativen Cotton-Effekt bei 212 und $278 \mathrm{~nm}$ im CD-Spektrum gekennzeichnet. Stereochemisch handelt es sich demnach um das gleiche Konformer wie 51. Der Metabolit ist in dieser Form noch nicht als Naturstoff beschrieben.

\section{6-Methoxyparvulenon ( $\underline{56})$}

Die in Form eines farblosen Öles erhaltene Substanz färbt mit Anis blaugrau an und ist UV-löschend. Das Protonenspektrum ist gekennzeichnet durch das Auftreten von zwei Methylengruppen bei $\delta_{H}=2.03 / 2.27$ und 2.56/2.80, einer Methylgruppe $\left(\delta_{H}=1.45\right)$, einer Methoxyfunktion $\left(\delta_{H}=3.88\right)$ und drei Methingruppen. Die Tieffeld-Verschiebung der $\mathrm{CH}$-Gruppen bei $\delta_{\mathrm{H}}=4.77$ und 5.18 läßt Rückschlüsse auf ein benachbartes Sauerstoffatom zu. Durch die Ansiedlung eines Protons bei $\delta_{H}=6.68$ kann dieses als aromatisch angesehen werden. Das 
Singulett bei $\delta_{\mathrm{H}}=13.19$ läßt sich einer Hydroxygruppe zuordnen. Im ${ }^{13} \mathrm{C}-\mathrm{NMR}$ Spektrum werden die Ergebnisse des ${ }^{1} \mathrm{H}-\mathrm{NMR}$-Spektrums bestätigt, und es lassen sich weitere sechs quartäre Kohlenstoffatome bei $\delta_{C}=110.3,118.6,148.4,161.4$, 162.9 und 203.3 einordnen. Die Signale um 160 ppm weisen auf phenolische C-Atome hin und $\delta_{C}=203.3$ ist typisch für eine Ketofunktion.

Mit Hilfe der ${ }^{1} \mathrm{H}$-Kopplungen und des $\mathrm{H}, \mathrm{H}$-COSY-Experimentes lassen sich Teilstrukturen erkennen, die durch die C,H-Korrelationen im HMBC-Spektrum zu einem Molekül mit Dihydro-Naphthalenonstruktur zusammengefügt werden konnten. Auffällig ist, dass in den 1D-NMR-Spektren einige Signale als doppelter Satz mit minimaler Verschiebung vorliegen. Daraus konnte auf das Vorliegen eines Dimers geschlossen werden.

Die Molmassenpeaks in der ESI-MS von 509 $\left([\mathrm{Dimer}+\mathrm{Na}]^{+}\right)$und $995 \quad\left([2 \mathrm{Dimer}+\mathrm{Na}]^{+}\right)$ stehen im Einklang mit dieser Vermutung. $275\left([252+\mathrm{Na}]^{+}\right)$steht für den MonomerMassenpeak. Eine Hochauflösung von 509 lieferte eine Summenformel von $\mathrm{C}_{26} \mathrm{H}_{15} \mathrm{O}_{4}$. Demzufolge müssten zwei Monomere über<smiles>[R]O[C@H]1CCC(=O)c2c1cc(OC)c([C@@H](C)O[R])c2O</smiles>
$\underline{53}$ eine Sauerstoffbrücke verbunden sein.

Nun stellte sich die Frage, welche der beiden möglichen Sauerstoffe das Brückenatom bildet. Um diesen Sachverhalt zu klären, bot es sich an, die Verbindung zu derivatisieren. Eine Umsetzung zum Acetat würde zu einer TieffeldVerschiebung der benachbarten Protonen führen. Die Umsetzung von $\underline{\mathbf{5 6}}$ mit Essigsäureanhydrid/Pyridin lieferte nach zwei Stunden zwei Produkte, die über Säulenchromatographie an Sephadex LH-20 (Aceton) in Reinsubstanz erhalten werden konnten.

Im ESI-Massenspektrum der Verbindung $\underline{\mathbf{5 4}}$ liegen die lonen bei 317 $\left([\text { Monomer+Na }]^{+}\right), 611\left([\text { Dimer+Na }]^{+}\right)$und $293\left([\text { Monomer-H }]^{-}\right)$. Das ${ }^{1} \mathrm{H}-\mathrm{NMR}-$ Spektrum zeigt die zusätzliche Acetoxygruppe bei $\delta_{H}=2.07$. Ein Tieffeldshift $(\Delta \delta=1.21 \mathrm{ppm})$ von $4-\mathrm{H}$ im Vergleich mit $\underline{\mathbf{5}}$ deutet auf dessen Entschirmung durch die entstandene Acetoxygruppe hin. Die Acetylierung erfolgte also an 4-OH, die Sauerstoffbrücke wäre dann an C-9 angesiedelt. 
Beim zweiten Produkt $\underline{\mathbf{5}}$ handelt es sich um ein Diacetat. Zwei neue Signale im ${ }^{1} \mathrm{H}-N M R-S p e k t r u m\left(\delta_{\mathrm{H}}=1.97\right.$ und 2.07$)$ stammen von den Acetoxyfunktionalitäten. Die tieffeldverschobenen Protonen $\left(\delta_{H}=5.97\right.$ und 6.27) weisen auf die acetylierten Hydroxygruppen an den Positionen 4 und 9 hin. Massenspektrometrische Untersuchungen (ESI) lieferten den erwarteten Massenpeak für das diacetylierte Monomer.<smiles>[R]OC(C)c1c(OC)cc2c(c1O)C(=O)CCC2OC(C)=O</smiles><smiles>COc1cc2c(c(O)c1C(C)OC(C)=O)C(=O)CCC2OC(C)=O</smiles>

Das diacetylierte Produkt führt zu dem Ergebnis, dass es sich bei $\underline{\mathbf{5}} \mathbf{6}$ nicht um ein Dimer handeln kann, da eine Etherspaltung unter den angewandten Reaktionsbedingungen kaum möglich ist. Durch El-MS-Experimente wurden die positiven Monomermassen bestätigt und die ESI-MS-Ergebnisse neu zugeordnet.

\begin{tabular}{|c|c|}
\hline Massenpeak & richtige Zuordnung \\
\hline \multicolumn{2}{|l|}{$\underline{56}$} \\
\hline 275 & {$[\mathrm{M}+\mathrm{Na}]^{+}$} \\
\hline 509 & {$\left[2 \mathrm{M}-\mathrm{H}_{2} \mathrm{O}+\mathrm{Na}\right]^{+}$} \\
\hline 995 & {$\left[4 \mathrm{M}-2 \mathrm{H}_{2} \mathrm{O}+\mathrm{Na}\right]^{+}$} \\
\hline \multicolumn{2}{|l|}{$\underline{54}$} \\
\hline 317 & {$\left[\mathrm{M}^{\prime}+\mathrm{Na}\right]^{+}$} \\
\hline 611 & {$\left[2 \mathrm{M}^{\prime}-\mathrm{H}_{2} \mathrm{O}+\mathrm{Na}\right]^{+}$} \\
\hline
\end{tabular}<smiles>COc1cc2c(c(O)c1C(C)O)C(=O)CCC2O</smiles>

$\underline{56}$

Tabelle 6: Korrigierte Zuordnung der ESI-Massenpeaks für $\underline{\mathbf{5}}$ und das acetylierte Derivat $\underline{\mathbf{5 4}}$.

Dennoch existieren Doppelsignale in den NMR-Spektren. Um einer Erklärung näher zu kommen, wurden die Unterschiede in den Verschiebungen der einzelnen Atome nochmals betrachtet. Auffällig waren die Aufspaltungen im linken oberen 
Molekülteil, besonders die Protonenverschiebung der phenolischen OH-Gruppe und die der C-Atome in den Positionen 4a, 6, 8 und 9.

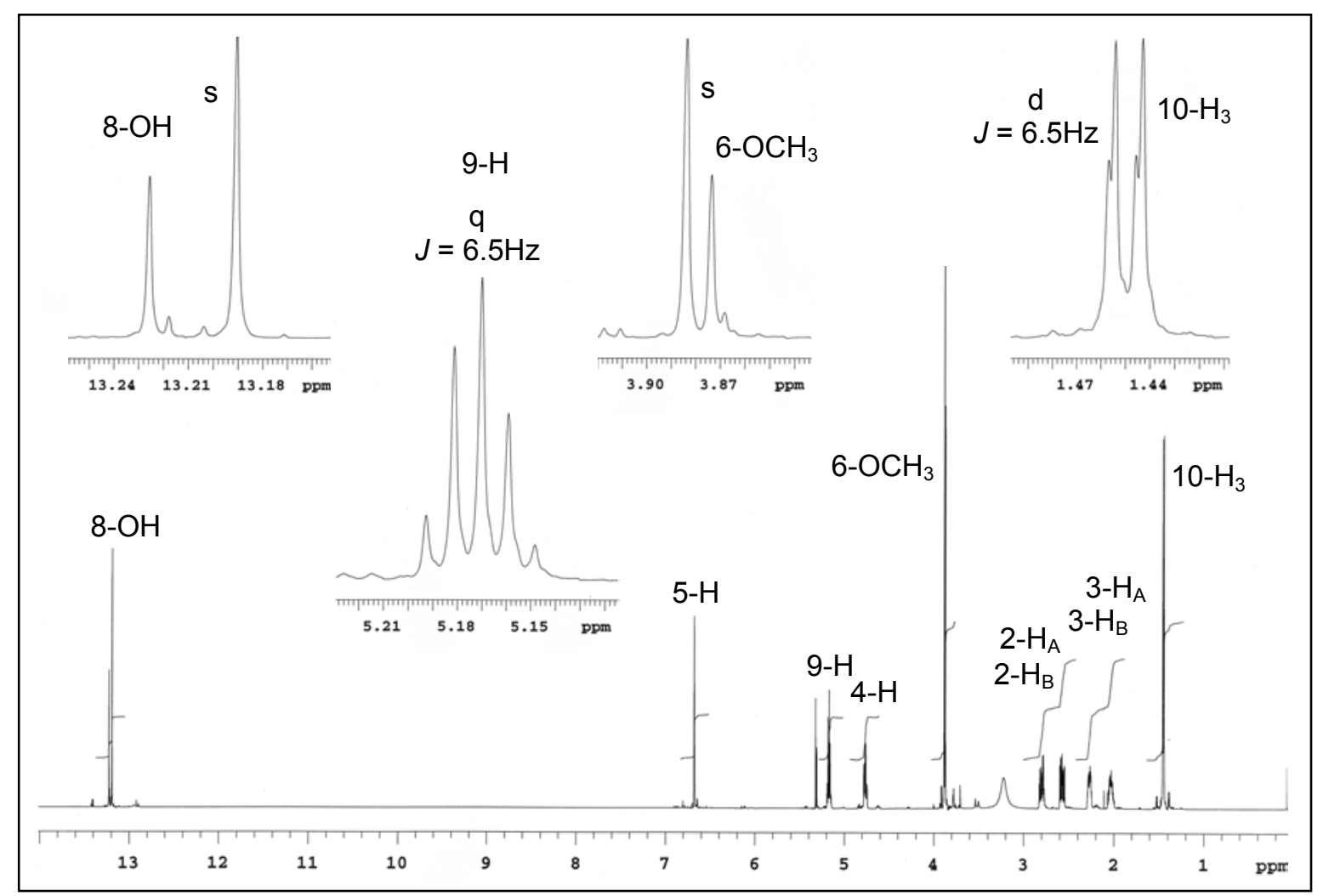

Abbildung 19: 'H-NMR-Spektrum für 6-Methoxyparvulenon (트) und ausgewählte Spreizungen.

Eine mögliche Erklärung kann die Umorientierung einer Wasserstoffbrücke sein, so dass zwei stabile Konformere entstehen. Die Umgebungsänderung der betroffenen Atome führt dann zu der Variation in den chemischen Verschiebungen. Die nachfolgende Zeichnung soll dies verdeutlichen.<smiles>COc1cc2c3c(c1C(C)O[B]O3)OCCC2O</smiles>

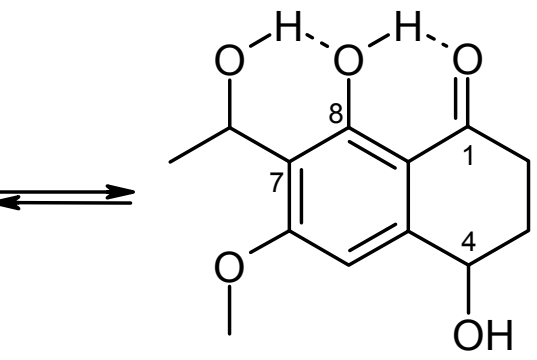

Eine Datenbankrecherche in $\underline{\text { Antibase }}^{47}$ und $\underline{\text { SciFinder }}^{76}$ führte zu keinem Treffer. Die Verbindung stellt einen neuen Naturstoff dar. Führt man die Suche nach Strukturmerkmalen etwas großzügiger durch, gelangt man zu einigen Hits. 


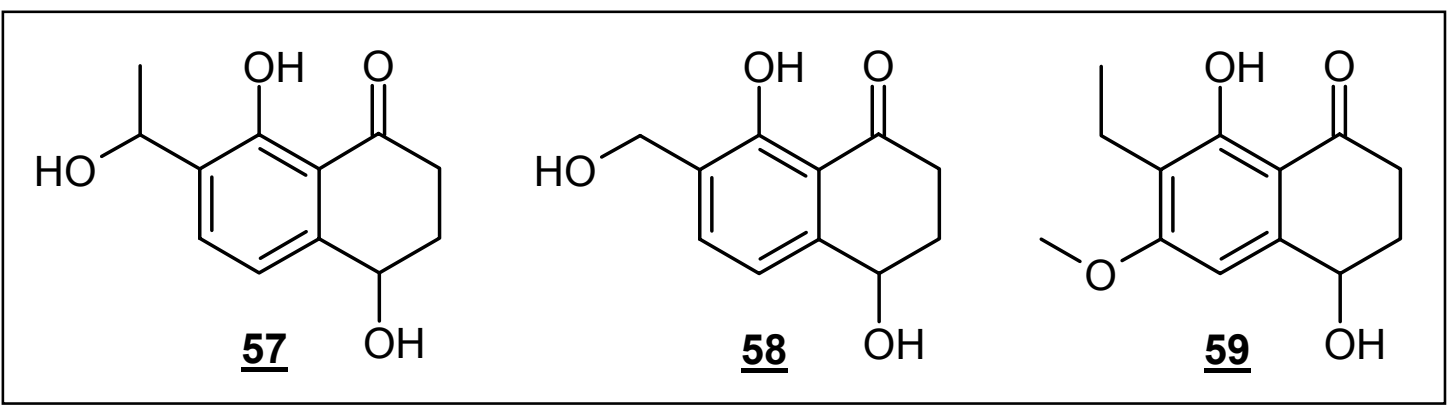

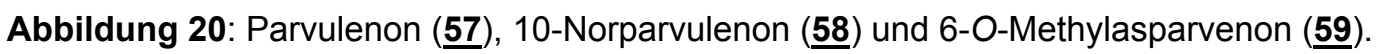

Die isolierte Verbindung kann als 6-Methoxyparvulenon eingeordnet werden. Parvulenon ( $\underline{\text { 57}}$ ) wurde erstmals 1979 aus Aspergillus parvulus isoliert. ${ }^{91} 21$ Jahre später wurde in einem Screening nach Anti-Influenza-Virus-Aktivität das 10Norparvulenon ( $\underline{\mathbf{5 8}}$ ) im Kulturfiltrat einer Microsphaeropsis sp. gefunden, ${ }^{92}$ welches auch antimikrobiell wirkt. Einen weiteren Metaboliten mit dieser Grundstruktur stellt das Asparvenon, ${ }^{93}$ ebenfalls aus Aspergillus parvulus, dar, das als O-methyliertes Derivat $(\underline{\mathbf{5 9}})^{94}$ als erster Stickstoff-freier Serotonin (5HT)-Antagonist ${ }^{95}$ angesehen wird.

\section{Naphthalacton A $(\underline{60})$}

Der mit Anis blau anfärbenden Substanz ließ sich mittels ESI-MS eine Molmasse von $322 \mathrm{~g} / \mathrm{mol}$ zuordnen. Das IR-Spektrum indiziert das Vorhandensein einer Hydroxyl- $\left(3429 \mathrm{~cm}^{-1}\right)$, einer Carbonyl- $\left(1621 \mathrm{~cm}^{-1}\right)$ und einer Esterfunktion $\left(1779 \mathrm{~cm}^{-1}\right)$.

Aus den ${ }^{1} \mathrm{H}-\mathrm{NMR}$-Daten kann wie bei den vorherigen Substanzen auf eine Dihydronaphthalenon-Grundstruktur geschlossen werden. In den zwei Methylengruppen $\left(\delta_{H}=2.21 / 2.41,2.79 / 2.83\right)$, dem Methin-Signal bei $\delta_{H}=5.22$, den Methylsignalen bei $\delta_{H}=1.48,3.91$ und der Hydroxygruppe $\left(\delta_{H}=13.25\right)$ spiegelt sich das 6-Methoxyparvulenon ( $\underline{\mathbf{5 6}}$ ) wieder. Weiterhin zeigen sich zwei zusätzliche Signale. Die durch die Nachbarschaft eines Sauerstoffatoms tieffeldverschobene $\mathrm{CH}$ Gruppe bei $\delta_{\mathrm{H}}=4.76$ zeigt vincinale Kopplungen zu den Signalen einer Methyleneinheit $\left(\delta_{H}=2.24\right.$ und 2.99).

Das ${ }^{13} \mathrm{C}-N M R-S p e k t r u m$ bestätigt die 6-Methoxyparvulenon-Struktur. Zusätzlich werden die Signale einer $\mathrm{CH}_{2}$-Gruppe $\left(\delta_{C}=41.1\right)$ und zwei quartärer Kohlenstoffe 
detektiert. Die Verschiebung von $\delta_{C}=176.4$ spricht für eine Carboxylgruppe und $\delta_{C}=81.7$ lässt für dieses C-Atom die Nachbarschaft zu einem Heteroatom vermuten.

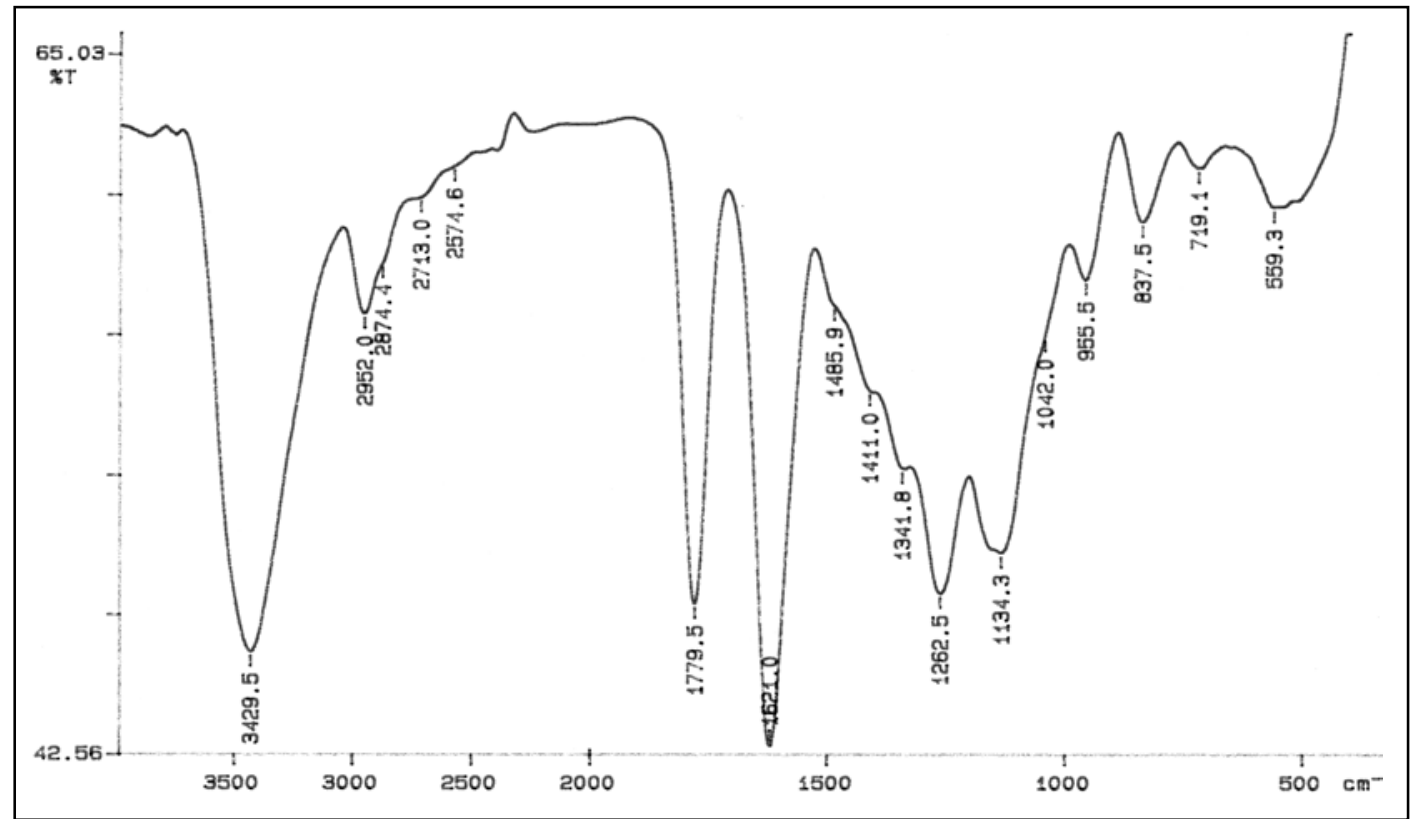

Abbildung 21: IR-Spektrum von $\underline{\mathbf{6 0}}$.

In Anlehnung an die NMR-Daten von $\underline{\mathbf{5 6}}$ ist der Grundkörper um einen LactonRing erweitert. Im H,H-COSY-Experiment sind zwei Kopplungssysteme auszumachen, wovon die längere Kette mit einer Fernkopplung von $1 \mathrm{~Hz}$ über ein quartäres Kohlenstoff-Atom läuft. Die Auswertung des HMBC-Experimentes bestätigt den Strukturvorschlag für Naphthalacton A $(\underline{\mathbf{6 0}})$.<smiles>[Z10][C@H]1CCC(=O)c2c1cc(OC)c(C(C)O)c2O</smiles>

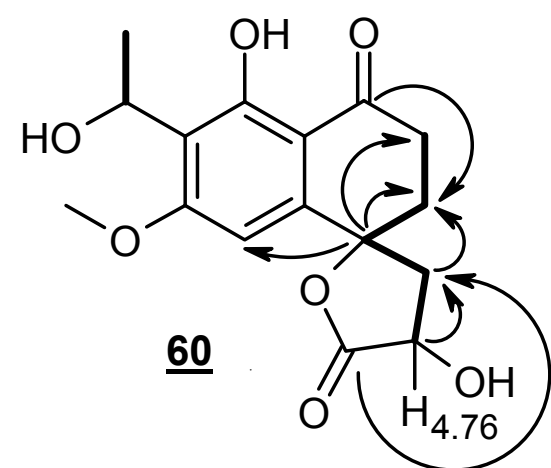

Abbildung 22: COSY-Fragmente (dick) und ausgewählte HMBC-Korrelationen von $\underline{56}$ und $\underline{60}$. 


\section{Naphthalacton B $(\underline{61})$}

Die in Form eines farblosen Öles erhaltene Substanz ist UV-löschend und färbt mit Anis braun an. Schon der erste Blick auf die NMR-Spektren implizierte ein Derivat von 60. Im ${ }^{13} \mathrm{C}-N M R-S p e k t r u m$ sind zwei Signale zu höherem Feld verschoben. Bei $\delta_{C}=12.93$ (statt 22.85) handelt es sich um die Methylgruppe in 10-Position. Anstatt der $\mathrm{CH}-$ Gruppe bei $\delta_{C}=63.6$ tritt ein Signal $\left(\delta_{C}=15.6\right)$ auf, das mit Hilfe eines APTs als $\mathrm{CH}_{2}$-Gruppe eingeordnet wird. Die Signale im ${ }^{1} \mathrm{H}-\mathrm{NMR}$-Spektrum stehen im Einklang mit dem ${ }^{13} \mathrm{C}-\mathrm{NMR}$. Die an Position 10 befindliche Methylgruppe erscheint hochfeldverschoben $\left(\delta_{\mathrm{H}}=1.05\right.$ statt 1.48) und zeigt ein Triplett mit einer Kopplungskonstanten von $7.5 \mathrm{~Hz}$. Den Kopplungspartner weist das Signal bei $\delta_{H}=2.62$, ein Quartett mit<smiles>CCc1c(OC)cc2c(c1O)C(=O)[C@H](O)C[C@]21CCC(=O)c2ccccc21</smiles>
$J=7.5 \mathrm{~Hz}$ und der Intensität von zwei Protonen. An die Dihydroxy-NaphthalenonGrundstruktur ist neben dem Lacton-Ring an C-4 ein Ethylrest an C-7 zu finden, wie inn auch das 6-O-Methylasparvenon (모) zeigt. Die Summenformel von $\mathrm{C}_{16} \mathrm{H}_{18} \mathrm{O}_{6}$ wird durch den Massenpeak bei $307[\mathrm{M}+\mathrm{H}]^{+}$im ESIMassenspektrum bestätigt. Die Struktur der Naphthalactone erwies sich in $\underline{\text { SciFinder }}^{76}$ als unbekannt.

Die Suche nach einer ähnlichen Struktur mit Lactonring führte zu nebenstehendem Naturstoff. Cochliospicin A (므) wurde aus dem Pilz Cochliobolus spicifer isoliert. ${ }^{96}$ Biosynthetisch leitet es sich vom Speciferinon ab, an dessen Polyketidgerüst eine $\mathrm{C}_{3}$-Einheit addiert wird, die den Lactonring bildet. $^{97}$ In<smiles>CC[C@]1(C)C(=O)C2=C(C(=O)[C@]13CC(O)C(=O)O3)[C@H](C)[C@@H](C)OC2</smiles>
$\underline{62}$ einem Bioassy mit Protoplasten von Weizenkeimblättern zeigte Speciferinon phytotoxische Aktivität, Cochliospicin A (므) jedoch nicht. Die Addition der $\mathrm{C}_{3}$-Einheit geht demzufolge mit dem Verlust der Phytotoxizität einher. Der Lactonring eröffnet damit neue Perspektiven zur Struktur-Wirkungs-Beziehung. 
In der neuesten Literatur von 2004 wird von<smiles>CC1=C[C@]2(CC(=O)c3c(O)cccc3C2O)OC1=O</smiles>
einem neuen Dihydronaphthalenon mit Spirofuranon-Struktur berichtet. ${ }^{98}$ Lambertellol A und B (63) wurden aus einer Lambertella Spezies isolierte und stellen Stereoisomere dar. Die absolute Stereochemie wurde nach chemischer Derivatisierung auf der Basis von CD-Spektren ermittelt.

\subsection{Biogeneseuntersuchungen}

Aufgrund der interessanten Spirolacton-Struktur der Naphthalactone wurden Biogeneseuntersuchungen an diesem Stamm angestrebt.

Ausgehend vom Cochliospicin A (2) kann man für die Metabolite $\underline{\mathbf{6 0}}$ und $\underline{\mathbf{6 1}}$ einen ähnlichen Biosyntheseweg mit Polyketid-Precursor und Addition einer $\mathrm{C}_{3}$-Einheit erwarten.

\subsubsection{Biosynthese-Experimente aus der Literatur}

An dieser Stelle helfen schon bekannte Biogeneseuntersuchungen an 6-O-Methylasparvenon (도). Fütterungsexperimente mit $\left[1-{ }^{13} \mathrm{C}\right]-, \quad\left[2-{ }^{13} \mathrm{C}\right]-, \quad\left[1,2-{ }^{13} \mathrm{C}_{2}\right]$ Acetat belegen den Einbau von sechs Acetat-Einheiten (-). ${ }^{99}$

Um den linearen Hexaketid-Precurser in $\underline{59}$ zu überführen, muss, neben dem Verlust der aus Acetat stammenden Sauerstoffe an den Positionen 3 und 9, ein Sauerstoffatom an C-4 eingefügt werden und die Reduktion zum Dihydronaphthalenon erfolgen. Um Einblicke in die Oxidations- und Reduktionsprozesse zu erhalten, wurde zusätzlich $\left[{ }^{2} \mathrm{H}_{3}\right]$ Acetat gefüttert. ${ }^{100}$ Eine Anreicherung $(\bullet)$ der 10-Methyl-, 5- und 2axialen Wasserstoffe war zu erwarten. Der positive Einbau der 3-axialen $\mathrm{H}$ wurde mit einem N.I.H.-shift<smiles>CCc1c(O)c2c(c(O)c1CC)C(O)[C@@H](C)CC2=O</smiles>

$\underline{59}$ während der Hydroxylierung von Tri- zum TetraHydroxynaphthalenon erklärt, wie er beim Pilzmetabolismus einiger aromatischer Substrate nachgewiesen wurde. ${ }^{101}$ 


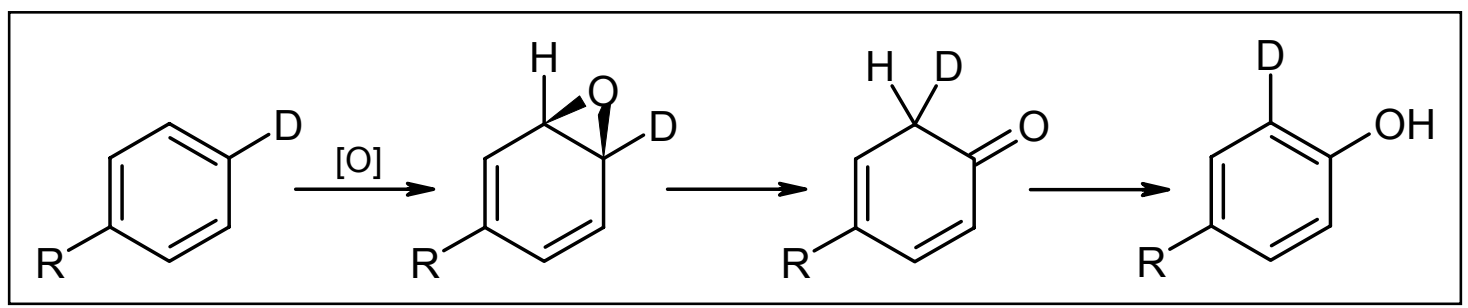

Abbildung 23: Bei der N.I.H.-Verschiebung handelt es sich um eine von WITKOP 1967 im National Institute of $\underline{\text { Health }}$ (Name) gefundene Umlagerung, die bei der enzymatischen Hydroxylierung von aromatischen Substraten auftritt.

Jüngere Biosynthesestudien belegen, dass 6-O-Methylasparvenon (모) über eine Pentaketid-Kette aufgebaut wird und die Ethyl-Seitenkette erst in einem späteren Biosyntheseschritt ans Molekül addiert wird. Das Auffinden eines Derivates mit einer Methyl-Seitenkette aus dem gleichen Stamm stützt diese Theorie. ${ }^{102}$

Von größerem Interesse ist jedoch die Bildung des $\gamma$-Lacton-Ringes. Marticin $(\underline{64})^{103}$ aus Fusarium marticiella und Canescin $(\underline{65})^{104}$ aus Aspergillus malignus bzw. Penicillium canescens stellen neben dem Cochliospicin A (2) weitere polyketidische Pilzmetabolite dar, die durch Addition einer $\mathrm{C}_{3}$-Einheit vervollständigt werden. In beiden Fällen wird davon ausgegangen, dass der Ursprung der $\mathrm{C}_{3}$-Einheit im Citratcyclus liegt.

In Fütterungsexperimenten am Marticin (여) mit doppelt markiertem Acetat $\left(1,2-{ }^{13} \mathrm{C}_{2}\right)$ waren alle Kohlenstoffatome mit Ausnahme der Methoxygruppen markiert. ${ }^{105} \mathrm{C}-16$ und $\mathrm{C}-17$ konnten als intakte Acetateinheit $(-)$ identifiziert werden, während C-15 nur angereichert ( $\square$ ) vorlag. Für C-15, C-16 und C-17 war die Anreicherung mit ca. 1\% deutlich niedriger als in den anderen Positionen (mind. 2\%). Es wurde postuliert, dass an ein Heptaketid Succinat, Oxalacetat oder ein anderer Metabolit aus dem Citratcyclus als gescrambeltes Acetat addiert wird.

Ein positiver Einbau von $\left[2-{ }^{13} \mathrm{C}\right]$-Acetat zeigte neben der Markierung im Polyketidgerüst eine Anreicherung an Position 15 und 16, was einem Einbau von Acetat in Succinat nach ein oder mehreren Durchgängen im Citratcyclus gleichkommt.

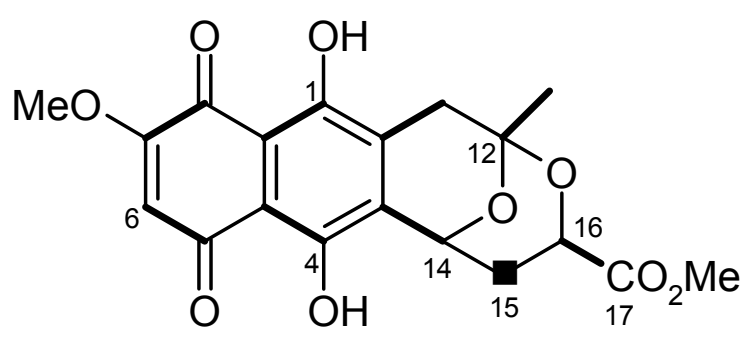

$\underline{64}$ 
<smiles>CO[C@@H]1C[C@H](c2c(O)cc3cc(C)oc(=O)c3c2O)OC1=O</smiles>

$\underline{65}$

Ähnliche Effekte konnten durch Fütterungsexperimente am Canescin (65) beobachtet werden. ${ }^{106}$ Der Einbau von $\left[1-{ }^{14} \mathrm{C}\right]$ Acetat, $\left[2-{ }^{14} \mathrm{C}\right]$ Acetat, Diethyl-[1,3- $\left.-{ }^{14} \mathrm{C}_{2}\right]$ malonat und $\left[{ }^{14} \mathrm{CH}_{3}\right]$ Methionin zeigte, dass das Isocoumarin-Gerüst dem Polyketid-Stoffwechsel entstammt und $\mathrm{C}-10$ des Lactonringes wie die Methoxygruppe ihren Ursprung in

Methionin $(\bullet)$ haben. Die ${ }^{14} \mathrm{C}$-Anreicherung durch markiertes Acetat und Diethylmalonat war im Lactonring deutlich geringer als im Isocoumarin-Gerüst, z.B. lag die Einbaurate von Diethyl-[1,3- $\left.{ }^{14} \mathrm{C}_{2}\right]$ malonat im Polyketid-System um $19 \%$, an C-13 nur bei $5.6 \%$, so dass auch bei $\underline{\mathbf{6} 5}$ nicht von einem direkten Einbau von Acetat bzw. Diethylmalonat ausgegangen werden konnte. Fütterungsexperimente mit ${ }^{14} \mathrm{C}$-Hydrogencarbonat und $\left[1-{ }^{14} \mathrm{C}\right]$ Pyruvat, nach denen kein ${ }^{14} \mathrm{C}$ Einbau in Canescin (65) zu verzeichnen war, führten zur Überlegung, dass die drei Kohlenstoffatome $\mathrm{C}-11, \mathrm{C}-12$ und $\mathrm{C}-13$ des Lactoninges eine symmetrische $\mathrm{C}_{4^{-}}$ Säure aus dem Zitronensäurecyclus als Vorläufer haben. Diese Vermutung wurde durch den Einbau von $\left[3-{ }^{14} \mathrm{C}\right]$ Äpfelsäure, $\left[3-{ }^{14} \mathrm{C}\right]$ Asparaginsäure und $\left[2,3-{ }^{14} \mathrm{C}_{2}\right]-$ Bernsteinsäure bestätigt.

Vorläufer-dirigierte Biosynthese mit deuterierten Verbindungen wiesen $\underline{68}$ als Biosyntheseintermediat aus und führten zum postulierten Biosyntheseschema in Abbildung 24. ${ }^{107}$

Der Isocoumarin-Grundkörper $\underline{\mathbf{6}} \underline{\mathbf{6}}$ ist das Produkt einer Polyketid-Synthase. Oxidation der Methylgruppe von $\underline{\mathbf{6 7}}$ führt zum Aldehyd $\underline{\mathbf{6 8}}$, welcher eine Aldolreaktion mit Oxalacetat eingeht, es wird $\underline{\mathbf{6 9}}$ erhalten. Die nachfolgenden Schritte einer Decarboxylierung und Lactonisierung können durch unterschiedliche Mechanismen auf zwei Wegen (A und B) über die Zwischenprodukte $\underline{70}$ oder $\underline{71}$ erfolgen. Die späte Biosynthesesequenzen beinhalten die Reduktion der Ketogruppe zum Alkohol $\underline{73}$ und eine anschließende Methylierung zum Canescin (ㅁ5). 


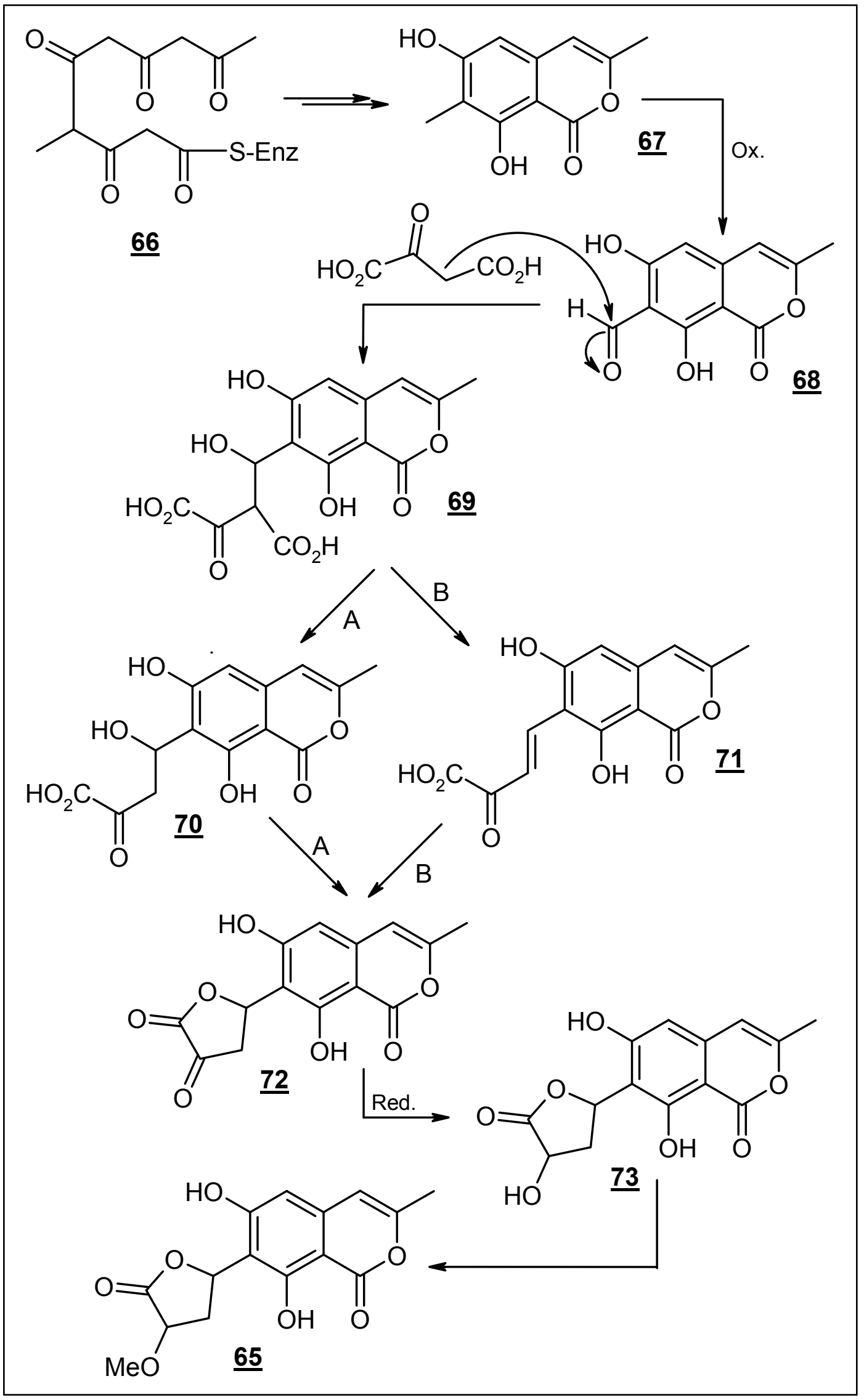

Abbildung 24: Postulierter Biosyntheseweg für Canescin $(\underline{65}){ }^{107}$ 


\subsubsection{Eigene Biogeneseuntersuchungen}

\subsubsection{Fermentationskurve}

Für Biosyntheseuntersuchungen ist es wichtig, bei einem gegebenen Stamm den zeitabhängigen Produktionsverlauf der Sekundärmetabolite zu kennen. Erkenntnisse über den $\mathrm{pH}-$ Verlauf und die Produktbildung liefern notwendige Hinweise, um die Länge der Kultivierungszeit festlegen und markierte Vorläufer gezielt zum richtigen Zeitpunkt in die spezifischen Stoffwechselwege eines Sekundärmetaboliten einschleusen zu können, damit eine genügend hohe Anreicherung im Zielmolekül erreicht wird. Daher erfolgt die Fütterung der markierten Vorläufer meist zu Beginn der Sekundärstoffproduktion und endet mit dem Erreichen der maximalen Konzentration eines Metaboliten.

Zur Aufnahme einer Fermentationskurve wurde der Stamm über sieben Tage in Schüttelkolben kultiviert und ab der 42. Stunde arbeitete man alle $12 \mathrm{~h}$ jeweils zwei Kolben standardisiert auf. Die erhaltenen Kulturfiltrat-Extrakte wurden dünnschichtchromatographisch und mit Hilfe der HPLC 1 (Säule 2, Programm 2) untersucht.

Bei der Analyse der Dünnschichtchromatogramme ergab sich die Sekundärstoffproduktion aus der Intensität der UV-Löschung bei $254 \mathrm{~nm}$ und $365 \mathrm{~nm}$ und aus der Intensität der Farbreaktion mit Anisaldehyd.

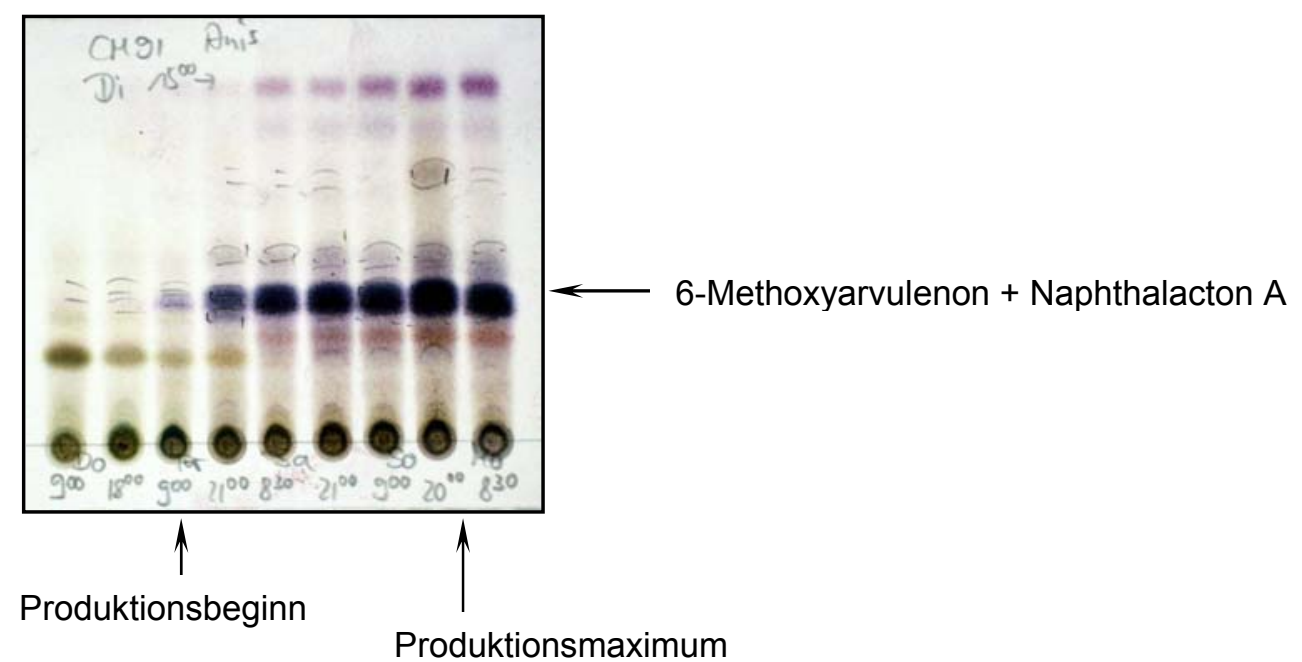

Abbildung 25: Zeitabhängige Produktbildung des Stammes WDMH28 (DC-Kieselgel) von der 42. bis zur 138. Stunde. 


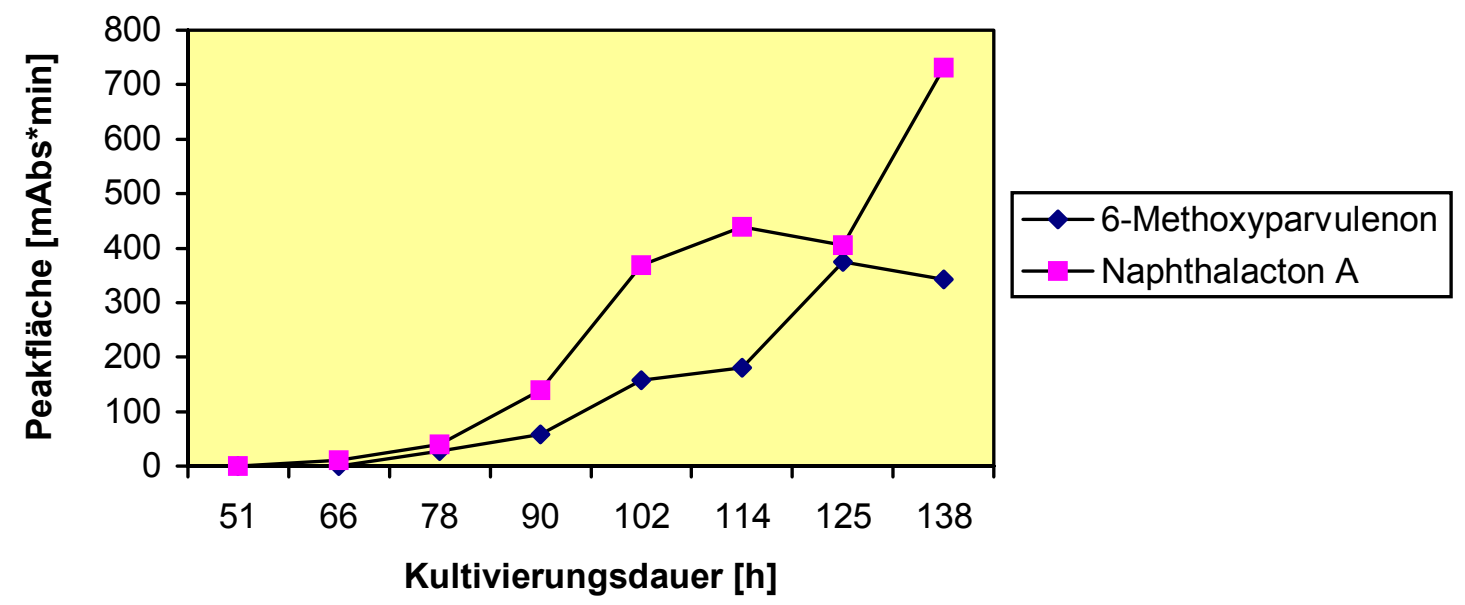

Abbildung 26: Fermentationskurve von 6-Methoxyparvulenon (트) und Naphthalacton A (으) aus WDMH28.

Bei der Analyse der Fermentationskurve wurden nur die beiden Hauptmetabolite 6-Methoxyparvulenon ( $\underline{\mathbf{5 6}})$ und Naphthalacton A $(\underline{\mathbf{6 0}})$ betrachtet.

Es ist deutlich zu erkennen, dass die Sekundärstoff-Produktion um die 66. Stunde einsetzt, dann kontinuierlich ansteigt und nach 125 Stunden erste Abbaureaktionen einsetzen. Deshalb wurde bei allen Fütterungsexperimenten die PrecursorLösung ab der 66. Inkubationsstunde mittels Puls-Feeding-Verfahren über $36 \mathrm{~h}$ zugegeben und die Fermentation nach der 114. Stunde abgebrochen.

\subsubsection{Fütterungsexperimente an 6-Methoxyparvulenon ( $\underline{56})$}

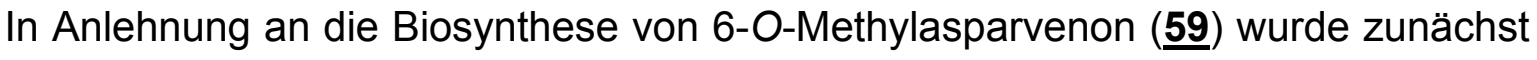
Acetat als Grundbaustein des Polyketidweges als Vorläufer ausgewählt.

Dem Stamm WDMH28 wurden in verschiedenen 1 L-Ansätzen als Schüttelkultur $750 \mathrm{mg}\left[1-{ }^{13} \mathrm{C}\right]$ Acetat, $500 \mathrm{mg}\left[1,2-{ }^{13} \mathrm{C}_{2}\right]$ Acetat und $919 \mathrm{mg}\left[2-{ }^{13} \mathrm{C}\right]$ Malonsäure (200 mL Lösung) zugefüttert. Nach standardisierter Aufarbeitung (Abbildung 4)

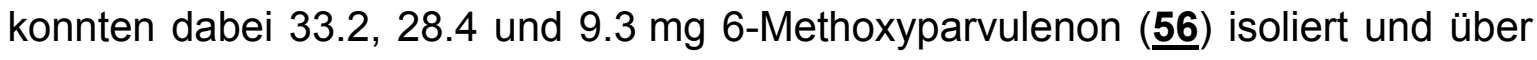
${ }^{13} \mathrm{C}-N M R-T e c h n i k$ vermessen werden. Zur Auswertung des Versuches wurde als Referenzsignal das Kohlenstoffatom der 6-Methoxygruppe ausgewählt, das bei dem Fütterungsexperiment keine Anreicherung aufweisen sollte. 


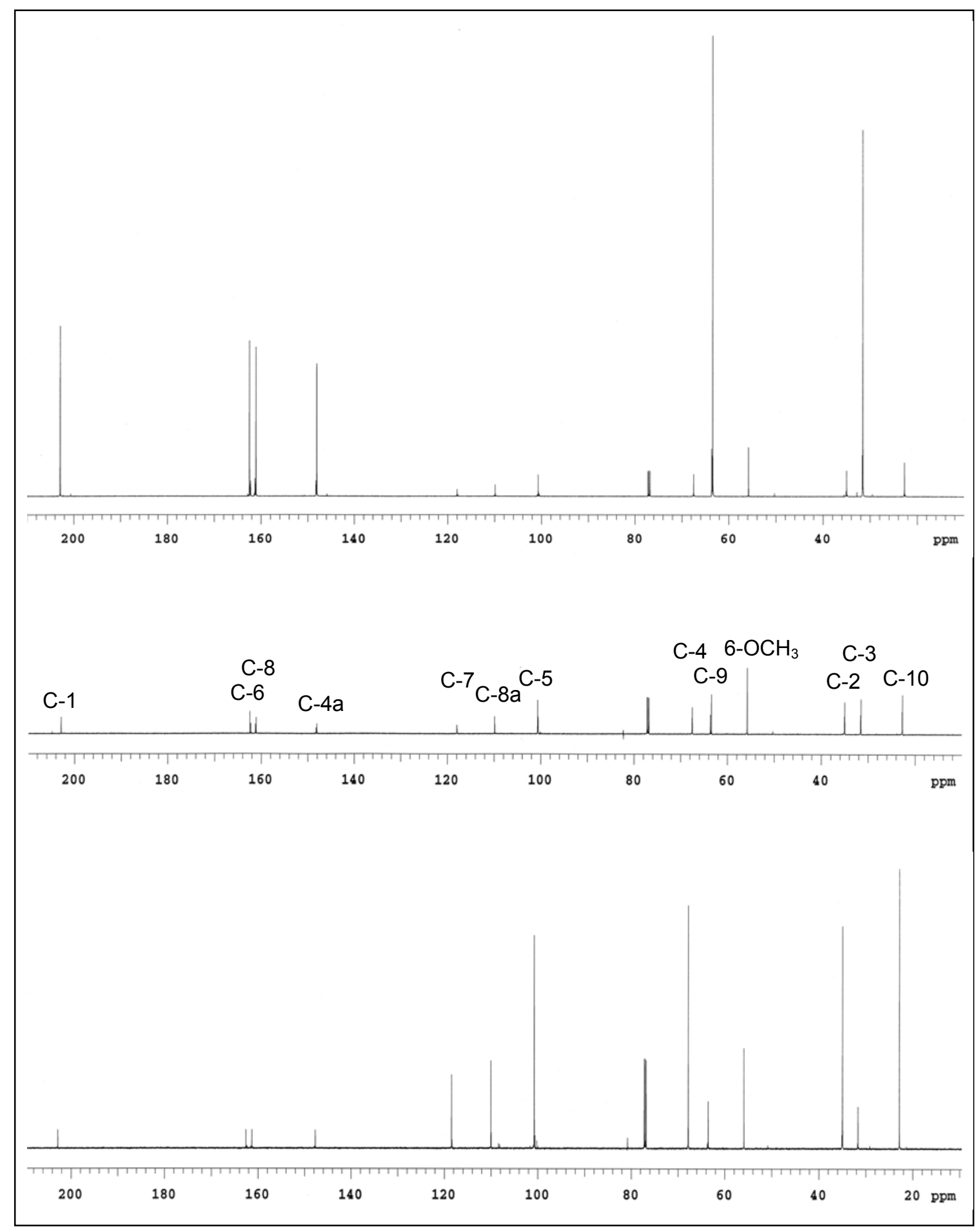

Abbildung 27: ${ }^{13} \mathrm{C}-\mathrm{NMR}$-Spektrum von 6-Methoxyparvulenon $(\underline{\mathbf{5 6}})\left(125 \mathrm{MHz}, \mathrm{CDCl}_{3}\right)$ aus den Fütterungsexperimenten mit einfach markierten Verbindungen. oben: $\left[1-{ }^{13} \mathrm{C}\right]$ Acetat, mitte: Referenz, unten: $\left[2-{ }^{13} \mathrm{C}\right]$ Malonsäure. 
Im ${ }^{13} \mathrm{C}-N M R-S p e k t r u m$ der aus $\left[1-{ }^{13} \mathrm{C}\right]$ Acetat angereicherten Substanz (Abbildung 27 ) ließen sich beachtliche Intensitätserhöhungen für C-1, C-3, C-4a, C-6, C-8 und C-9 erkennen, mit einem spezifischen Einbau von 9.8 bis 20.6. Unter Berücksichtigung der natürlichen Häufigkeit von ${ }^{13} \mathrm{C}-K e r n e n$ sowie der Anreicherung des Precursors gilt ein Einbau als erfolgreich, wenn die Signalintensität eines C-Atoms verdoppelt und die Anreicherung somit größer eins ist.

$\%$ Anreicherung $=1.1 \% \times \frac{\text { Intensität des Signals der markierten Verbindung }}{\text { Intensität des Signals der unmarkierten Verbindung }}-1.1 \%$ spezifischer Einbau $=\frac{\% \text { Anreicherung } \times 100}{\% \text { Anreicherung des Vorläufers }}$

Damit steht außer Frage, dass Acetat bzw. Malonat direkt in das Zielmolekül

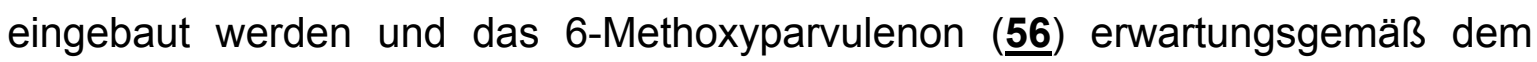
Polyketidstoffwechsel entstammt. Nach dem erfolgreichen Einbau von $\left[1-{ }^{13} \mathrm{C}\right]-$ Acetat, sollte mit dem Einbauversuch von doppelt markiertem $\left[1,2-{ }^{13} \mathrm{C}_{2}\right]$ Acetat geklärt werden, ob der Einbau der Acetatbausteine intakt erfolgt.

\begin{tabular}{|c|c|c|c|}
\hline C-Atom & $\delta$ in ppm & $\begin{array}{c}\text { spezifischer Einbau } \\
\text { aus }\left[1-{ }^{13} \mathrm{C}\right] \text { Acetat }\end{array}$ & $\begin{array}{c}\text { spezifischer Einbau } \\
\text { aus }\left[2-{ }^{13} \mathrm{C}\right] \text { Malonsäure }\end{array}$ \\
\hline 1 & 202.9 & $\mathbf{1 4 . 9 6}$ & -0.28 \\
\hline 2 & 34.9 & 0.11 & $\mathbf{4 . 6 7}$ \\
\hline 3 & 31.5 & $\mathbf{1 5 . 0 3}$ & -0.23 \\
\hline 4 & 67.5 & 0.13 & $\mathbf{5 . 6 4}$ \\
\hline $4 \mathrm{a}$ & 148.1 & $\mathbf{2 0 . 6 1}$ & 0.30 \\
\hline 5 & 100.7 & -0.15 & $\mathbf{3 . 5 5}$ \\
\hline 6 & 162.5 & $\mathbf{9 . 7 5}$ & -0.49 \\
\hline 7 & 117.9 & 0.11 & $\mathbf{5 . 3 9}$ \\
\hline 8 & 161.1 & $\mathbf{1 3 . 4 4}$ & -0.25 \\
\hline $8 \mathrm{a}$ & 109.9 & -0.09 & $\mathbf{2 . 7 3}$ \\
\hline 9 & 63.4 & $\mathbf{1 6 . 6 7}$ & -0.24 \\
\hline 10 & 22.7 & 0.19 & $\mathbf{4 . 1 4}$ \\
\hline $6-\mathrm{OMe}$ & 55.8 & 0 & 0 \\
\hline
\end{tabular}

Tabelle 7: Ergebnisse aus den Fütterungsexperimenten mit $\left[1-{ }^{13} \mathrm{C}\right]$ Acetat und $\left[2-{ }^{13} \mathrm{C}\right]-$ Malonsäure; spezifischer Einbau in 6-Methoxyparvulenon (흐); $\left(125 \mathrm{MHz}, \mathrm{CDCl}_{3}\right)$. 
Im ${ }^{13} \mathrm{C}-N M R-S p e k t r u m$ waren für alle C-Atome des 6-Methoxyparvulenon (흐) mit Außnahme der Methoxygruppe typische Kopplungsmuster von angereicherten ${ }^{13} \mathrm{C}-K e r n e n$ zu finden, die nach Auswertung der Kopplungskonstanten Aufschluß über die Nachbarschaft der Kohlenstoffatome gaben. Neben der erwarteten Aufspaltung zum Dublett waren weitere, an Intensität geringere Kopplungen zu beobachten. Diese statistischen Kopplungen rühren von angereicherten C-Atomen her, die zwar benachbart sind, aber nicht einer intakten Acetateinheit zugehören, also von der geringen statistischen Wahrscheinlichkeit für die Bildung eines Isotopomers, in das zwei markierte Acetateinheiten gleichzeitig eingebaut sind. Als Beispiel sei die statistische Kopplung zwischen C-2 und C-3 mit 29 Hz angeführt.

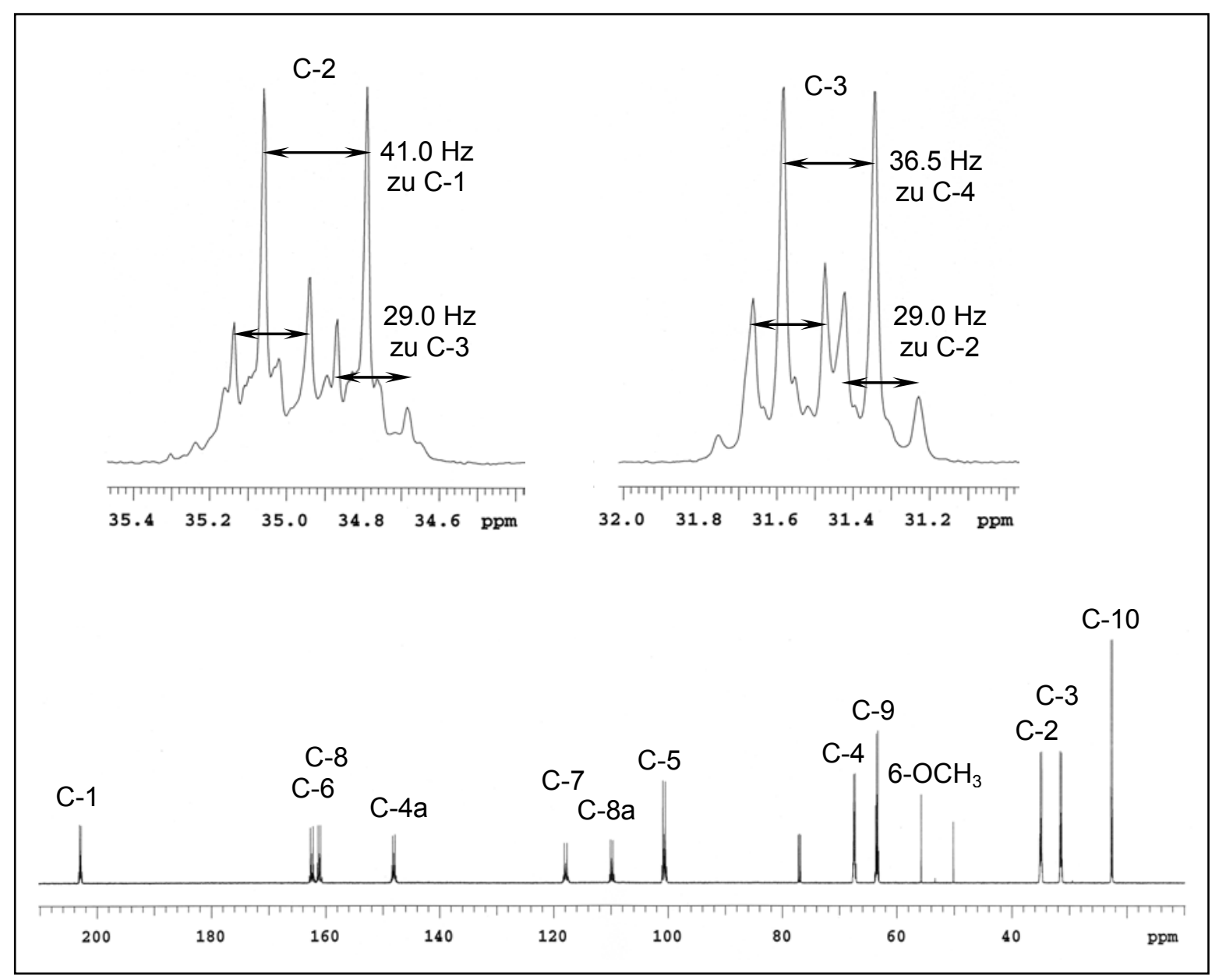

Abbildung 28: ${ }^{13} \mathrm{C}-\mathrm{NMR}-S p e k t r u m$ von $\underline{\mathbf{5 6}}\left(125 \mathrm{Mz}, \mathrm{CDCl}_{3}\right)$ aus der Fütterung mit $\left[1,2-{ }^{13} \mathrm{C}_{2}\right]$ Acetat mit Spreizungen für $\mathrm{C}-2$ und $\mathrm{C}-3$. 
Die ermittelte Einbaurichtung der sechs intakten Acetateinheiten konnte durch Fütterungsexperimente mit $\left[2-{ }^{13} \mathrm{C}\right]$ Malonsäure bestätigt werden.

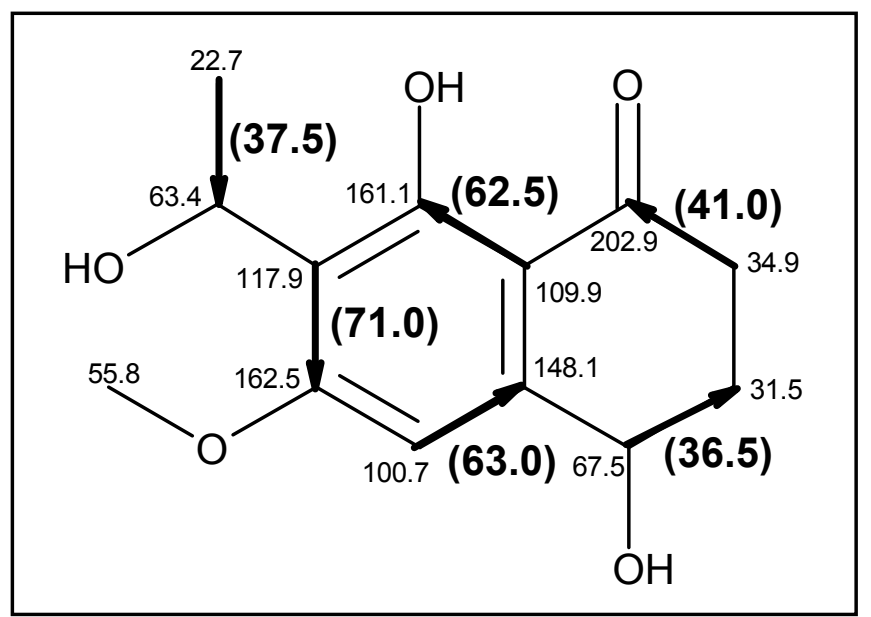

Abbildung 29: 6-Methoxyparvulenon ( $\underline{56})$ mit Kopplungskonstanten (fett, in Klammern) aus der $\left[1,2-{ }^{13} \mathrm{C}_{2}\right]$ Acetat-Fütterung

\subsubsection{Fütterungsexperimente an Naphthalacton $A(\underline{60})$}

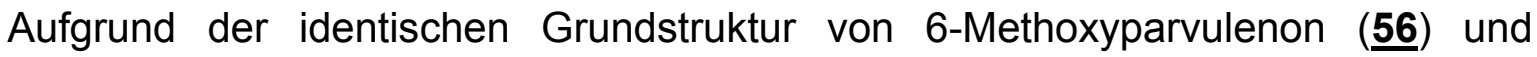
Naphthalacton A (우) wird davon ausgegangen, dass der DihydronaphthalenonKörper auch bei $\underline{60}$ über den gleichen Polyketidstoffwechsel aufgebaut wird. Im Hinblick auf den vorgestellten Lacton-Biosyntheseweg erwartet man die Addition eines $\mathrm{C}_{3}$-Körpers, der dem Zitronensäurecyclus entspringt. Bei einer Fütterung von markiertem Acetat würde man dementsprechend folgendes Einbaumuster erwarten.

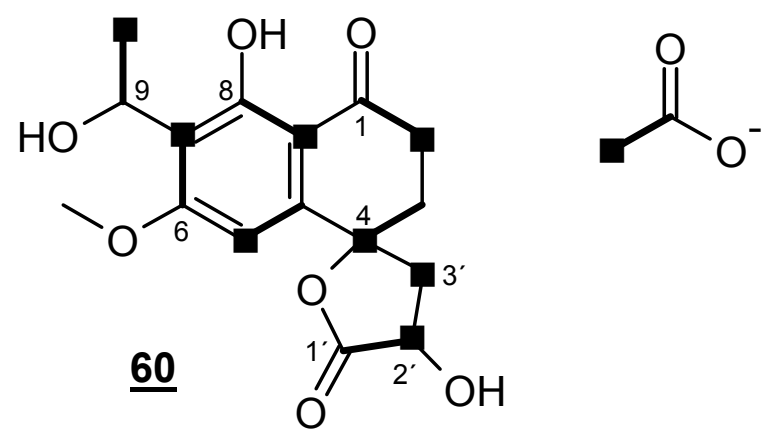


Um dies zu verifizieren, wurde der Stamm WDMH28 in verschiedenen $1 \mathrm{~L}$ Ansätzen in Schüttelkolben kutiviert und jeweils $750 \mathrm{mg}\left[1-{ }^{13} \mathrm{C}\right]$ Acetat, $500 \mathrm{mg}$ [1,2- $\left.{ }^{13} \mathrm{C}_{2}\right]$ Acetat und $919 \mathrm{mg} \quad\left[2-{ }^{13} \mathrm{C}\right]$ Malonsäure (200 mL Lösung) zugefüttert (Experiment vgl. Seite 54).

Die Aufarbeitung nach Standardbedingungen lieferten 8.9, 3.1 und $0.7 \mathrm{mg}$ Naphthalacton A $(\underline{\mathbf{6 0}})$. Die ${ }^{13} \mathrm{C}-N M R-S p e k t r e n$ dieser Verbindungen wurden mit dem der unmarkierten Verbindung verglichen. Als Referenzsignal diente wieder das Kohlenstoffatom der 6-Methoxygruppe, da es keine Anreicherung aufweisen sollte. Die $\left[1-{ }^{13} \mathrm{C}\right]$ Acetat-Fütterung führte zur Intensitätserhöhungen für die Kohlenstoffe 1, 3, 4a, 6, 8 und 9, aber nicht an C-1'. Dieses zeigt zwar eine höhere Anreicherung als die anderen unmarkierten Kohlenstoffatome, ist aber nicht als Einbau zu betrachten.

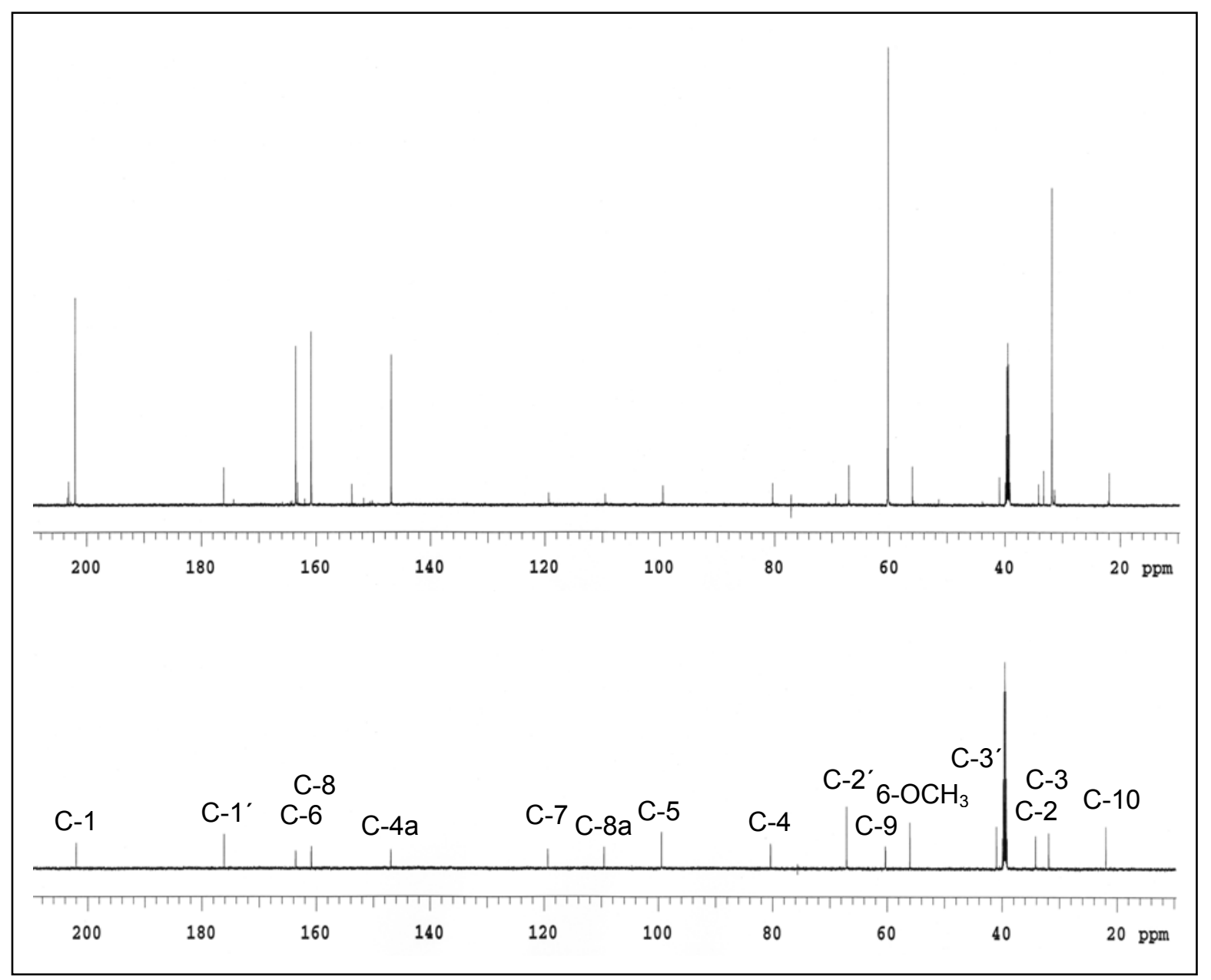

Abbildung 30: ${ }^{13} \mathrm{C}-\mathrm{NMR}-S p e k t r u m\left(125 \mathrm{MHz}\right.$, DMSO- $\left.\mathrm{D}_{6}\right)$ von $\left[1-{ }^{13} \mathrm{C}\right]$ Acetat-angereichertem Naphthalacton A $(\underline{\mathbf{6 0}})$ (oben), Referenz (unten). 
Auch die Malonat-Fütterung zeigte keine eindeutigen Ergebnisse bezüglich der Herkunft des Lacton-Ringes. Referenziert man alle Signale auf die Methoxygruppe, wirkt C-3' leicht angereichert, C-2' ist unmarkiert. Wird das CarbonylKohlenstoff C-1'als Referenz genutzt, beobachtet man sowohl für C-2' als auch für C-3' eine Erhöhung der Intensität. Allerdings muss dann auch das MethinKohlenstoff an C-9 als markiert angesehen werden.

\begin{tabular}{|c|c|c|c|c|}
\hline \multirow{2}{*}{ C-Atom } & \multirow{2}{*}{$\delta$ in ppm } & \multirow{2}{*}{\begin{tabular}{|c|} 
spezifischer Einbau \\
aus $\left[1-{ }^{13} \mathrm{C}\right]$ Acetat
\end{tabular}} & \multicolumn{2}{|c|}{ spez. Einbau aus $\left[2-{ }^{13} \mathrm{C}\right]$ Malonsäure } \\
\hline & & & Ref: 56.1 & Ref: 176.1 \\
\hline 1 & 202.0 & 6.90 & n.b. & n.b. \\
\hline 2 & 34.1 & -0.50 & 1.93 & 2.43 \\
\hline 3 & 31.9 & 7.65 & -0.18 & -0.02 \\
\hline 4 & 80.3 & -0.13 & 3.31 & 4.56 \\
\hline $4 a$ & 146.9 & 8.36 & n.b. & n.b. \\
\hline 5 & 99.4 & -0.60 & 1.07 & 1.41 \\
\hline 6 & 163.6 & 8.91 & 0.37 & n.b. \\
\hline 7 & 119.3 & -0.41 & 1.93 & 2.43 \\
\hline 8 & 160.9 & 9.17 & n.b. & n.b. \\
\hline $8 a$ & 109.5 & -0.37 & 1.30 & 1.69 \\
\hline 9 & 60.2 & 23.81 & 0.49 & 1.17 \\
\hline 10 & 21.9 & -0.18 & 2.15 & 3.64 \\
\hline 6-OMe & 56.1 & 0 & 0 & 0.18 \\
\hline $1^{\prime}$ & 176.1 & -0.05 & -0.17 & 0 \\
\hline $2^{\prime}$ & 67.05 & -0.49 & 0.03 & 0.73 \\
\hline $3^{\prime}$ & 40.9 & -0.47 & 0.68 & 0.97 \\
\hline
\end{tabular}

Tabelle 8: Ergebnisse aus den Fütterungsexperimenten mit $\left[1-{ }^{13} \mathrm{C}\right] \mathrm{Acetat}$ und $\left[2-{ }^{13} \mathrm{C}\right]$-Malonsäure;

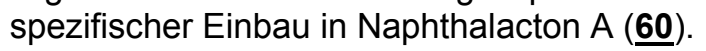

Als Probleme seien hier genannt, dass der Stamm die Substanz während der Malonsäure-Fütterung nur unzureichend produziert hat und diese sich nicht bis zur vollständigen Reinheit aufarbeiten ließ. So standen für die Untersuchungen nur $0.7 \mathrm{mg}$ des leicht verunreinigten Metaboliten zur Verfügung.

Nach den unklaren Ergebnissen der bisherigen Fütterungen, die Biogenese des Lactonringes betreffend, sollte der Einbauversuch von $\left[1,2-{ }^{13} \mathrm{C}_{2}\right]$ Acetat beweisen, dass der Einbau einer intakten Acetateinheit in den 5-Ring erfolgt. 
Nur zwei von sechzehn Signalen waren im ${ }^{13} \mathrm{C}-N M R-S p e k t r u m$ als Singulett zu erkennen. Bei den übrigen Signalen handelt es sich um Dubletts angereicherter ${ }^{13} \mathrm{C}-$ Kerne, zum Teil sind statistische Kopplungen erkennbar.

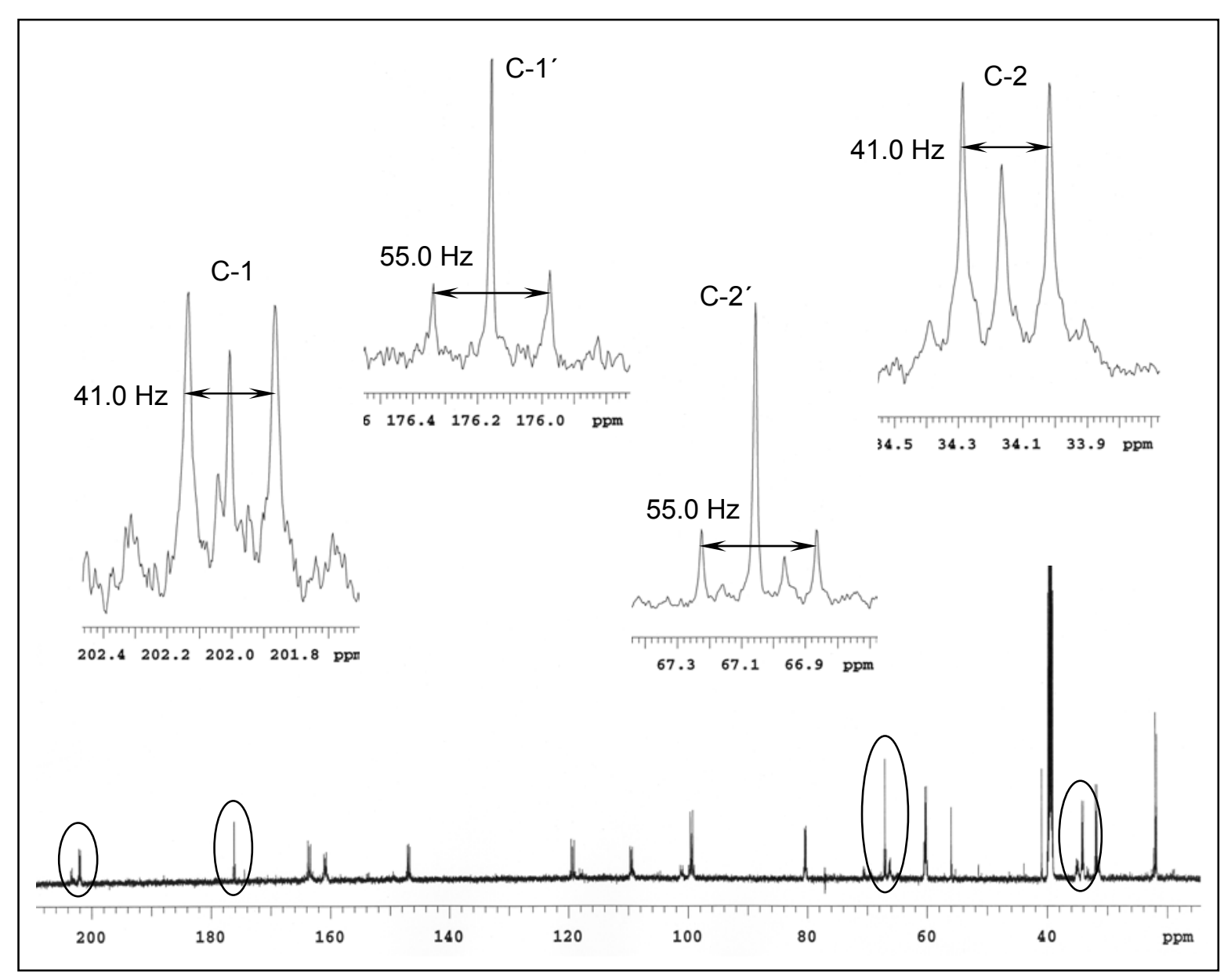

Abbildung 31: ${ }^{13} \mathrm{C}-\mathrm{NMR}-S p e k t r u m\left(125 \mathrm{MHz}\right.$, DMSO- $\left.\mathrm{D}_{6}\right)$ von Naphthalacton A $(\underline{\mathbf{6 0}})$ aus der Fütterung mit $\left[1,2-{ }^{13} \mathrm{C}_{2}\right]$ Acetat mit Spreizungen für C-1, C-2, C-1' und C-2'.

In Tabelle 9 sind die Kopplungskonstanten für die angereicherten C-Atome angegeben. Daraus ergibt sich, dass sechs intakte Acetateinheiten (C-1/C-2, C3/C-4, C-4a/C-5, C-6/C-7, C-8/C-8a und C9/C-10) in den Parvulenon-Körper eingebaut wurden und eine in $\mathrm{C}-1^{\prime} / \mathrm{C}-2^{\prime}$ des Lactonringes. Bei den letztgenannten Kohlenstoffatomen war die Signalintensität des Dubletts im Vergleich zur Zentrallinie deutlich geringer als bei den anderen markierten C-Atomen (Abbildung 31). Um zu prüfen, ob C-3' ebenfalls aus Acetat stammt, wurde das Singulett gegen das Kohlenstoffatom der Methoxygruppe referenziert und es 
konnte eine Anreicherung von $0.74 \%$ mit einem spezifischen Einbau von 0.75 bestimmt werden.

\begin{tabular}{|c|c|c|}
\hline C-Atom & $\delta$ in ppm & $\begin{array}{c}{ }^{1} J(\mathrm{C}, \mathrm{C})- \\
\text { Kopplungen in } \mathrm{Hz}\end{array}$ \\
\hline $1 / 2$ & $202.0 / 34.1$ & 41.0 \\
\hline $3 / 4$ & $31.9 / 80.3$ & 38.0 \\
\hline $4 \mathrm{a} / 5$ & $146.9 / 99.4$ & 63.5 \\
\hline $6 / 7$ & $163.6 / 119.3$ & 70.0 \\
\hline $8 / 8 \mathrm{a}$ & $160.9 / 109.5$ & 61.5 \\
\hline $9 / 10$ & $60.2 / 21.9$ & 38.0 \\
\hline $6-\mathrm{OMe}$ & 56.1 & - \\
\hline $1^{\prime} / 2^{\prime}$ & $176.1 / 67.05$ & 55.0 \\
\hline $3^{\prime}$ & 40.9 & - \\
\hline
\end{tabular}

Tabelle 9: Ergebnisse des Fütterungsexperimentes mit $\left[1,2-{ }^{13} \mathrm{C}_{2}\right]$ Acetat für das Naphthalacton A (60); ; Das ${ }^{13} \mathrm{C}-N M R-S p e k t r u m$ wurde bei $35^{\circ} \mathrm{C}$ in DMSO-D 6 gemessen $(125 \mathrm{MHz})$.

Die geringere Anreicherung im Lactonring bestätigt die Eindrücke aus der Literatur, dass Acetat nicht direkt, sondern über den Stoffwechselweg des Citratcyclus gescrambelt in das Molekül eingebaut wird.

Um dem Nachhalt zu verleihen, wurde in einem weiteren Experiment $\left[1,4-{ }^{13} \mathrm{C}_{2}\right]-$ Succinat (135 mg, $2.2 \mathrm{mmol} / \mathrm{L})$ der wachsenden Kultur zugefügt. Man erhielt $2.1 \mathrm{mg}$ des Naphthalacton A $(\underline{\mathbf{6 0}})$, in dessen ${ }^{13} \mathrm{C}-\mathrm{NMR}-S p e k t r u m$ nur ein Signal erhöht war, das der Lactoncarboxylgruppe. Die spezifische Anreicherung wurde mit 1.75 berechnet.

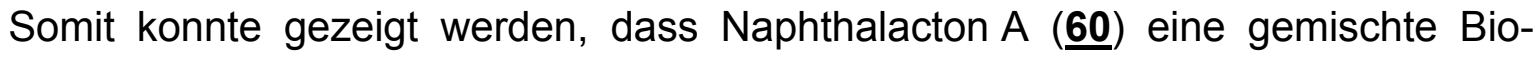
synthese aufweist. Die Bildung des Dihydronaphthalenon-Grundgerüstes erfolgt analog zu $\underline{56}$. Die Tatsache, dass auch Isoscleron (1) und 6-Methoxy-isoscleron (52) aus dem Stamm isoliert wurden, lässt darauf schließen, dass sich zunächst eine Pentaketidkette bildet. Erst nach Cyclisierung wird die Seitenkette an C-7 addiert und 6-OH methyliert. Mit der Addition einer $\mathrm{C}_{4}$-Säure aus dem Zitronensäure-Stoffwechsel wird die Bildung des Lactonringes eingeleitet und der Tricyclus in abschließenden Biosyntheseschritten zu $\underline{\mathbf{6 0}}$ vervollständigt. 


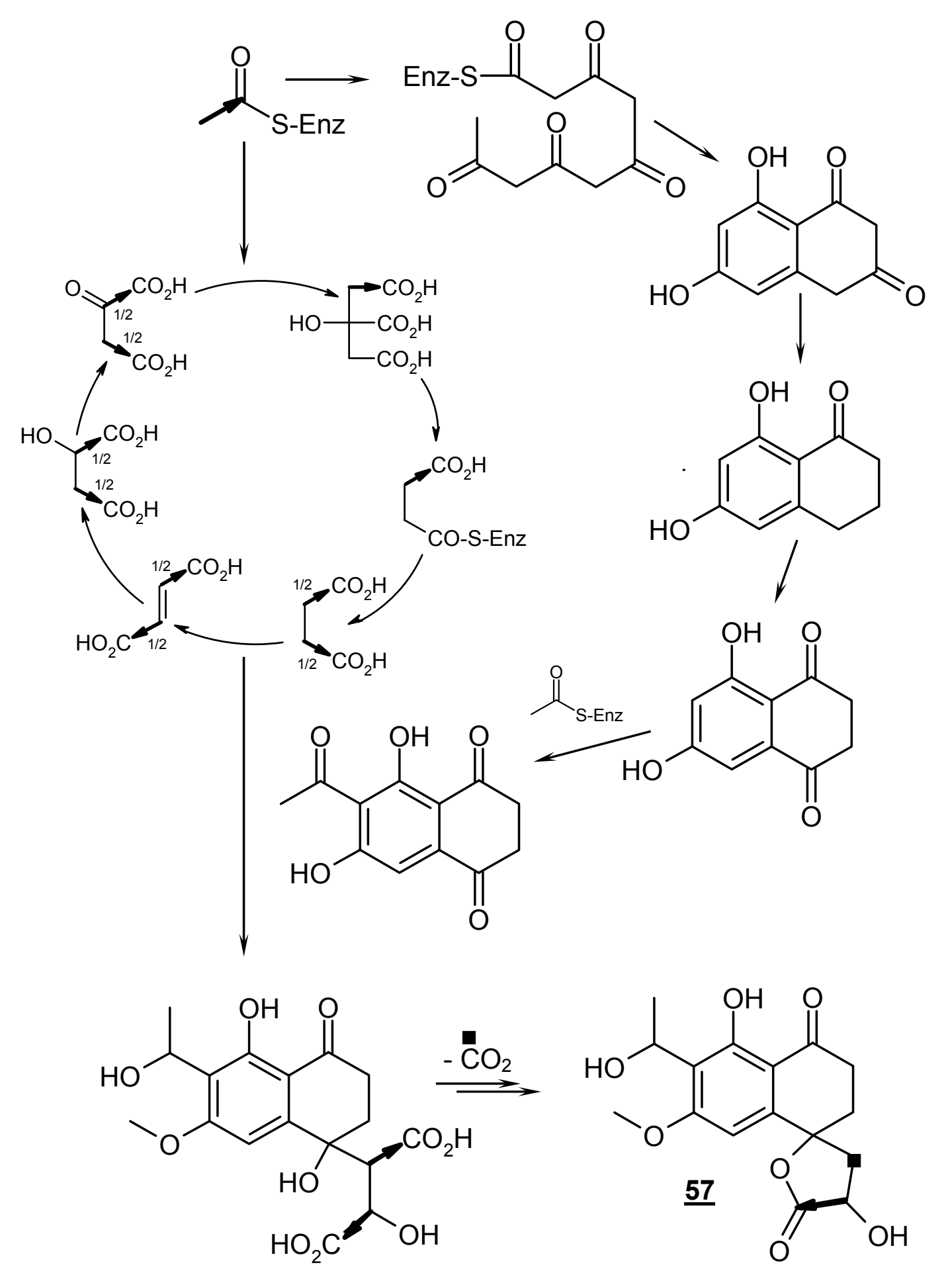

Abbildung 32: Postulierte Biosynthese für Naphthalacton $A(\underline{\mathbf{6 0}})$ und andere Derivate aus dem Stamm WDMH28. Es wird die Markierung aus Acetat für einen Durchgang des Citratcyclus berücksichtigt. 
Der Stamm WDMH28 ist bezüglich seiner Sekundärstoffproduktion als vielfältig zu beurteilen. Neben $\alpha$-Acetylorcinol ( $\underline{\mathbf{5 0}}$ ) bildet er eine Reihe von Dihydronaphthalenon-Derivaten, von denen vier $(\underline{\mathbf{5 2}}, \underline{\mathbf{5 6}}, \underline{\mathbf{6 0}}$ und $\underline{61})$ neu sind. Die TetralonGrundstruktur wird durch Addition einer Methoxygruppe an C-6, einer Seitenkette an C-7 und/oder einer $\mathrm{C}_{3}$-Einheit aus dem Citratcyclus an C-4 modifiziert.

Für keinen der Metabolite ist bislang eine biologische Aktivität gefunden worden.<smiles>CC(=O)Cc1cc(O)cc(O)c1</smiles>

$\underline{50}$ $\alpha$-Acetylorcinol

$(3.0 \mathrm{mg} / \mathrm{L})$<smiles>COc1cc2c(c(O)c1C(C)O)C(=O)CC[C@H]2O</smiles>

$\underline{56}$

6-Methoxyparvulenon (25.7 mg/L)<smiles>O=C1CC[C@H](O)c2cccc(O)c21</smiles>

$\underline{51}$ Isoscleron (1.6 mg/L)<smiles>COc1cc2c(c(O)c1C(C)O)C(=O)CCC21CC(O)C(=O)O1</smiles>
60 Naphthalacton A $(7.1 \mathrm{mg} / \mathrm{L})$<smiles>COc1cc(O)c2c(c1)[C@H](O)CCC2=O</smiles>

52

6-Methoxyisoscleron (1.2 mg/L)<smiles>CCc1c(OC)cc2c(c1O)C(=O)O[C@@]21CCC(=O)[C@@]1(C)CCO</smiles>

61

Naphthalacton B $(1.1 \mathrm{mg} / \mathrm{L})$

Abbildung 33: Sekundärmetabolite aus dem nicht identifizierten Pilzstamm WDMH28. 


\section{Stamm WDMH46}

\subsection{Allene}

Die Chemie der Allene ${ }^{108}$ begann im Jahr 1875 mit einer Publikation, ${ }^{109}$ in der VAN'T HOFF nicht nur das Tetraedermodell für Alkane, sondern auch die korrekten Strukturen für Allene und höhere Cumulene vorhergesagt hatte. Obwohl allgemeine Skepsis über die Stabilität kummulierter Doppelbindungssysteme herrschte, entstand ein reges Interesse auf diesem Arbeitsgebiet. Mit den bis 1950 bekannten Analysemethoden war es jedoch kaum möglich, zwischen Allenen und Alkinen zu unterscheiden. Erst nach Einführung der IR- und Raman-Spektroskopie konnte anhand der charakteristischen allenischen Valenzschwingung bei ca. $1950 \mathrm{~cm}^{-1}$ diese Struktureinheit zweifelsfrei nachgewiesen werden. Heutzutage sind etwa 150 Naturstoffe mit Allen- oder Cummulen-Struktur bekannt. ${ }^{110}$ Die meisten natürlichen Allene können in drei große Klassen eingeordnet werden: 1) lineare Allene, 2) allenische Carotinoide und Terpene sowie 3) Bromallene.

Die Gruppe der linearen Allene umfaßt mehr als 30 Verbindungen, ${ }^{111}$ die aus unterschiedlichen Quellen (Mikroorganismen, Pilzen, höhere Pflanzen, Insekten) isoliert wurden. Dazu zählt auch das Mycomycin (4), ein Pilzmetabolit, der als erster authentischer allenischer Naturstoff angesehen wird. ${ }^{112}$ Seine antibiotische Aktivität lässt sich allerdings schlecht nutzen, da seine Diinallen-Struktur nur eine geringe Beständigkeit aufweist und sich sehr leicht zum Dientriin Isomycomycin (프) umlagert. ${ }^{112 b}$

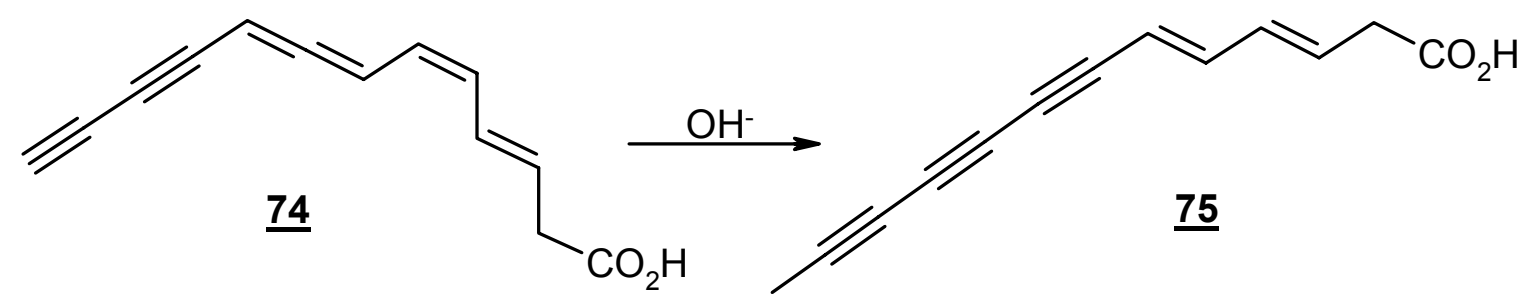

HORLER et al. entdeckten 1970 den Tetradeca-2,4,5-triensäuremethylester (두) in einem Bohnenkäfer der Spezies Acanthoscelides obtectus. ${ }^{113}$ Der Bohnenschädling enthielt beachtliche Mengen (ca. 0.5\%) des Insektenpheromons, das interessanterweise nicht enantiomerenrein, sondern mit $80 \%$ ee vorlag. Obgleich Synthesen sowohl für die racemische, ${ }^{114}$ als auch für die enantiomeren-angereicherte ${ }^{115}$ 
Verbindung bekannt sind, wird $\underline{76}$ häufig als Zielverbindung für die Erprobung neuer stereoselektiver Synthesemethoden chiraler Allene genutzt. ${ }^{116,117}$

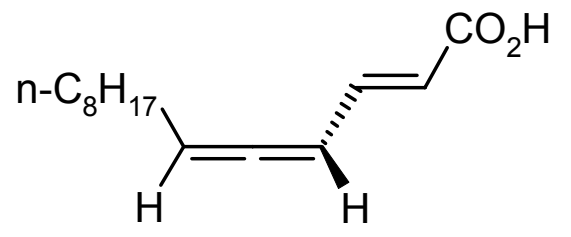

$\underline{76}$

Mit ca. 40 Verbindungen bilden Carotinoide und Terpene die größte Gruppe allenischer Naturstoffe. ${ }^{111}$ Den meisten Carotinoiden ist ein Cyclohexenyliden-Ring gemein, Unterschiede liegen in der Struktur und Peripherie des zweiten Ringes. Als typischer Vertreter sei neben dem "Heuschrecken-Keton" (프) das Fucoxanthin ( $\underline{77})$ genannt, das u.a. in Braunalgen und Diatomeen vorkommt. Obwohl schon 1914 entdeckt, ${ }^{118}$ gelang die Zuordnung der allenischen Struktur erst 50 Jahre später. ${ }^{119}$<smiles>CC(=O)O[C@H]1CC(C)(C)C(=C/C(C)=C/C=C/C(C)=C/C=C/C=C(C)/C=C/C=C(\C)C(=O)C[C@@]23O[C@]2(C)C[C@@H](O)CC3(C)C)[C@@](C)(O)C1</smiles>

Das "grasshopper ketone“ (프) wurde 1968 aus dem Abwehrsekret der großen flugunfähigen Heuschrecke Romalea microptera isoliert und wird wegen der Ähnlichkeit zum linken Molekülteil von $\underline{\mathbf{7 8}}$ als Abbauprodukt allenischer Carotinoide angesehen. ${ }^{120}$ Entsprechende in vitro-Untersuchungen stützen diese Hypothese. ${ }^{121}$

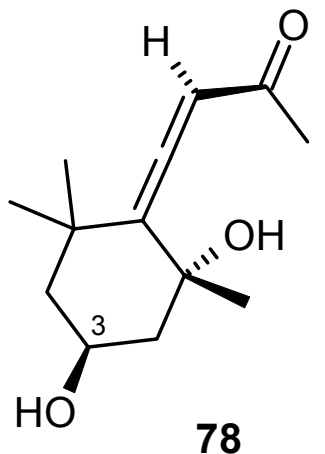

$\underline{78}$

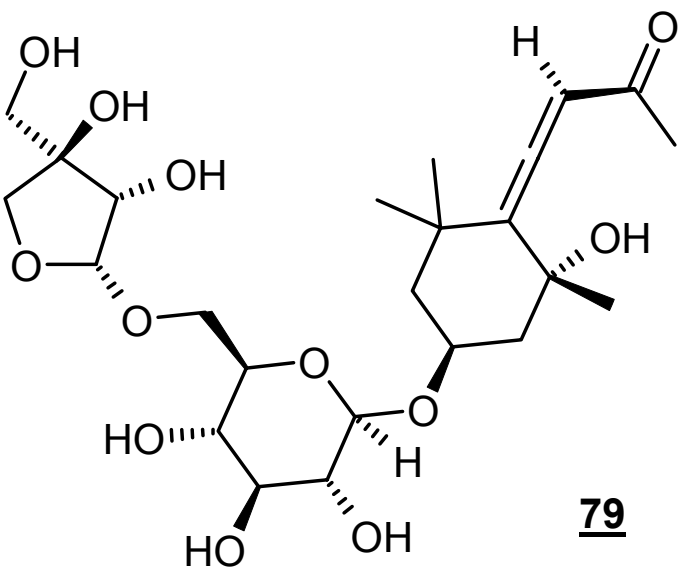


Ein in 3-Position glycosidisches Derivat (모), enthalten in Zimt (Cinnamomum cassia), wird in der asiatischen Volksmedizin als „Goreisan“ (China) oder „Kannan Keihi“ (Japan) bei Fieber und Schmerzen sowie bei Magenverstimmungen eingesetzt. ${ }^{122}$

Bei den über 30 bekannten Bromallenen ${ }^{111}$ handelt es sich größtenteils um $\mathrm{C}_{15^{-}}$ Dioxabicyclen mit exocyclischer Bromallengruppierung. ${ }^{123}$ Ein Isolat aus Aplysia brasiliana brachte den ersten Vertreter dieser Gruppe 1977 zu Tage. ${ }^{124}$ Das Panacen ( $\underline{\mathbf{8 0}}$ ) weist Eigenschaften als Fraßgift auf, aufgrund dessen die Molluske von Fischen verschmäht wird. Diese Naturstoffklasse ist in ihrer Struktur äußerst vielfältig. So findet man in Rotalgen auch Bicyclo[6.3.0]undecane wie das Laurallen (1) (aus Laurentia nipponica) ${ }^{125}$ mit einer 8-gliedrigen cyclischen Ethereinheit.

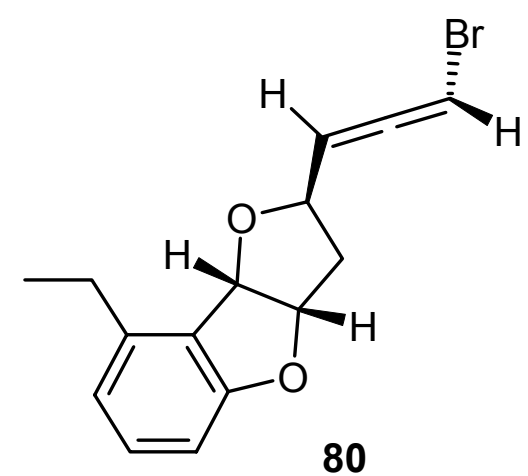

$\underline{80}$

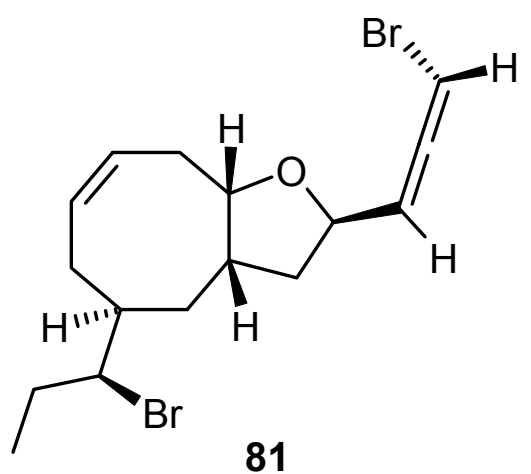

81

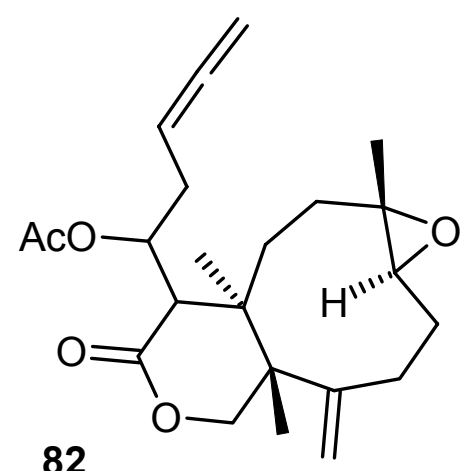

$\underline{82}$

Etwa 30 weitere Verbindungen lassen sich keiner der drei bisher behandelten Klassen zuordnen. ${ }^{111}$ Dazu zählen die Acalycixeniolide B-F mit terminaler Alleneinheit, die in Korallen der Gattung Acalycigorgia enthalten sind. ${ }^{126}$ Einige dieser Metabolite inhibieren das Wachstum von Seeigeleiern und wirken cytotoxisch gegen bestimmte Leukämie-Zelllinien. Für Acalycixeniolid E (요) wurde zudem eine antiangiogenetische Aktivität nachgewiesen. ${ }^{127}$ Auf der Haut des in Kolumbien beheimateten "Pfeilgiftfrosches" Dendrobates leucomelas fand man allenische Alkaloide. Das Isodihydrohistrionicotoxin $(\underline{\mathbf{8 3}})^{128}$ weist eine Azaspiro[5,5]undecan-Struktur auf und wirkt auf Ionenkanäle des Nicotin-AcetylcholinRezeptors. ${ }^{128 \mathrm{~b}}$ Von Decahydrochinolinen ${ }^{129}$ des Typs $\underline{84}$ wird angenommen, dass sie nicht vom Frosch gebildet, sondern über die Nahrung aufgenommen werden. Zum einen wurden diese Toxine nicht von in Gefangenschaft aufgezogenen Fröschen gebildet, zum anderen wurden ähnliche Verbindungen auch in der Ameise Solenopsis azteca gefunden. ${ }^{130}$ 


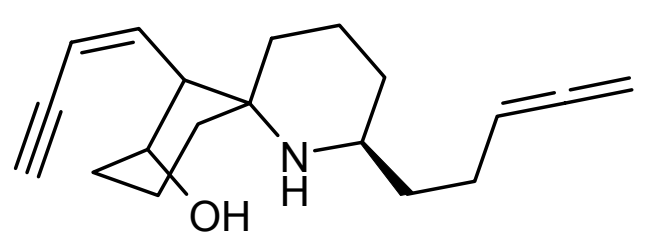

$\underline{83}$

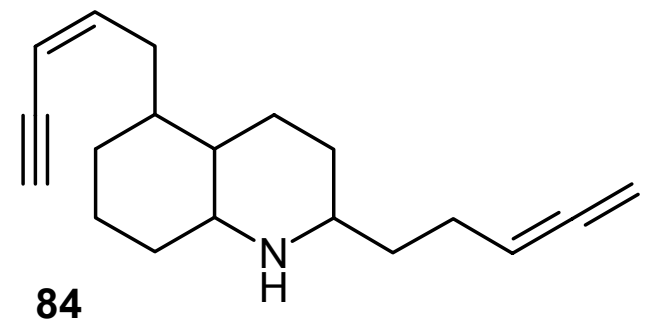

$\underline{84}$

Wegen der oftmals beeindruckenden biologischen Eigenschaften allenischer Naturstoffe sind Allengruppierungen mittlerweile systematisch in pharmakologisch aktive Verbindungsklassen (Steroide, Prostaglandine, Aminosäuren, Nucleoside) eingebaut worden. ${ }^{110}$ Diese funktionalisierten Allene weisen in vielen Fällen interessante Aktivitäten auf, beispielsweise als Mechanismus-inhärente Enzyminhibitoren, Cytostatika oder antivirale Wirkstoffe. ${ }^{110,111,131}$

Bislang wurden zwar vielfältige biologische Funktionen allenischer Naturstoffe beschrieben und es ist ebenso gelungen, die pharmakologische Wirkung aktiver Verbindungen durch den Einbau von Allengruppierungen zu erhöhen, aber es ist wenig Grundlegendes bekannt. Vielen Spekulationen über Struktur-WirkungsBeziehungen und Biogenese von Allenen stehen sehr wenige experimentelle Untersuchungen gegenüber. ${ }^{111}$

Im nachfolgenden Kapitel wird die Isolierung und Strukturaufklärung einiger neuer Allen-Metabolite beschrieben und auch über Biogenese-Experimente berichtet.

\subsection{Fermentation und Isolierung der gebildeten Metabolite}

Der Stamm WDMH46 fiel im chemischen Screening in den Nährmedien 1158, E2 und $\mathrm{G} 20$ durch die Bildung dreier bei $254 \mathrm{~nm}$ UV-löschender Zonen auf, die mit Anis pink anfärben. In den Medien 1549 und 1405 zeigt der Stamm ein ähnliches Metabolitenmuster, die Produktivität in diesen Medien war jedoch deutlich geringer. Aus einer Fermentation in Schüttelkolben (5L, NL 1158) ließen sich durch Aufarbeitung nach Abbildung 34 drei Metabolite in Ausbeuten von $27.7 \mathrm{mg}$ (흐), $9.8 \mathrm{mg}(\underline{\mathbf{8 5}})$ und $19.9 \mathrm{mg}(\underline{\mathbf{8 7}})$ gewinnen. Alle drei Verbindungen wurden als farbloses Öl erhalten und weisen in allen getesteten Laufmittelsystemen einen ähnlichen $\mathrm{R}_{\mathrm{f}}$-Wert auf. 


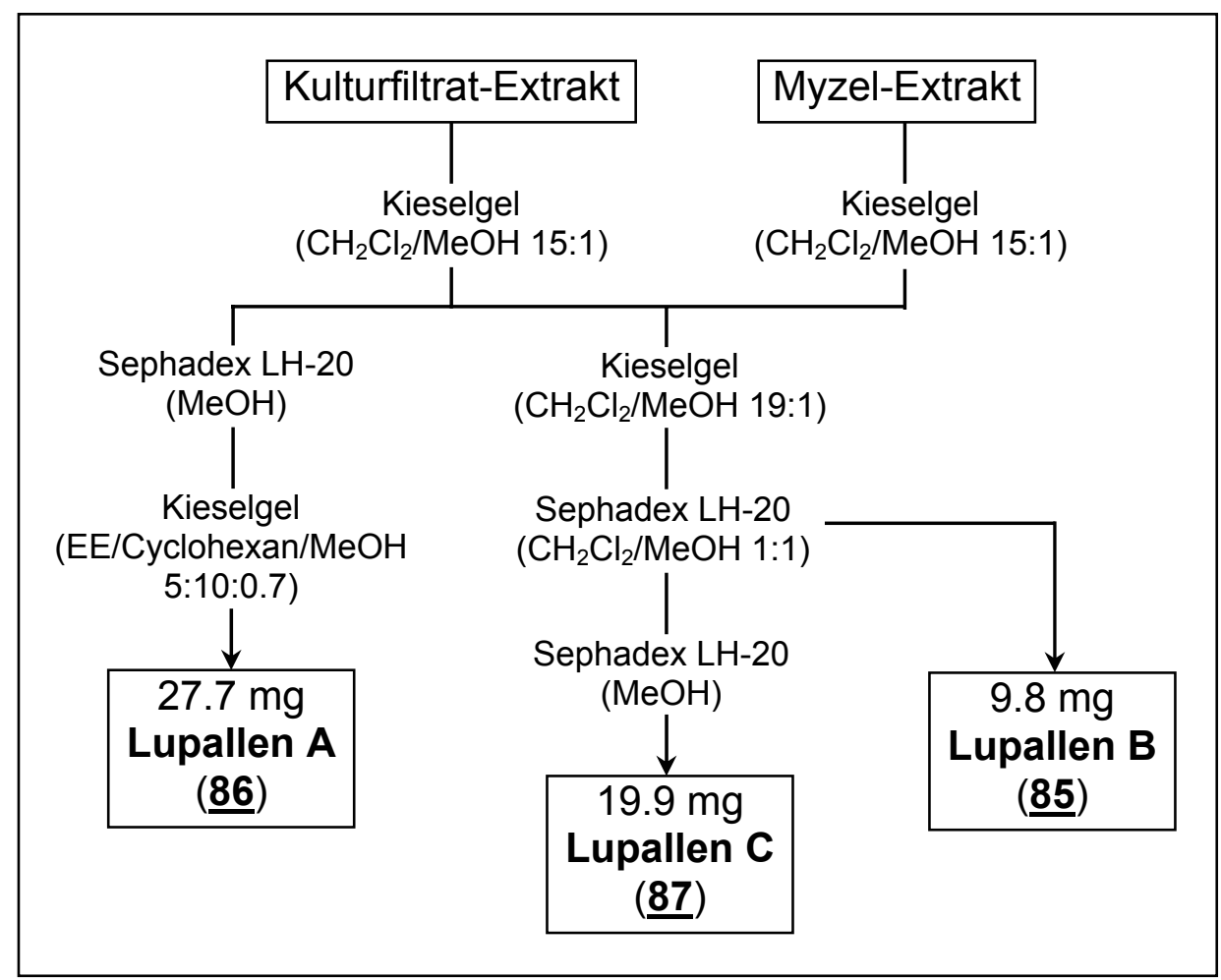

Abbildung 34: Aufarbeitungsschema für die Lupallene A-C (모-87).

\subsection{Charakterisierung der Lupallene}

\section{Lupallen B (모)}

Ein in Methanol- $\mathrm{D}_{4}$ aufgenommenes ${ }^{1} \mathrm{H}$-NMR-Spektrum von $\underline{85}$ weist Signale im Bereich von 1.8 bis 6.0 ppm auf. Im Tieffeldbereich sind die Resonanzen dreier olefinischer Protonen zu sehen, eines erscheint als Dublett $\left(\delta_{H}=5.95, J=3.5 \mathrm{~Hz}\right.$ ), die beiden anderen $\left(\delta_{\mathrm{H}}=4.81,4.89\right)$ weisen kleine Kopplungskonstanten um $1 \mathrm{~Hz}$ auf, die genaue Multiplizität lässt sich aber nicht einordnen. Die Kopplungskonstante von $3.5 \mathrm{~Hz}$ ist in dem Signal bei $\delta_{H}=2.31$ wiederzufinden, zugehörig zu einer Methylengruppe, deren zweites Proton bei $\delta_{H}=2.33(\mathrm{dd}, J=13.0,7.0 \mathrm{~Hz}$ ) auftaucht. Das Spinsystem wird durch eine Sauerstoff-substituierte Methingruppe ( $\delta_{\mathrm{H}}=3.91$, dd, $J=7.5,7.0 \mathrm{~Hz}$ ) ergänzt. In diesem Resonanzbereich sind drei weitere Signale angesiedelt, ein Singulett bei $\delta_{H}=4.34$ und ein um $1.0 \mathrm{~Hz}$ aufgespaltenes Dublett bei $\delta_{H}=3.02$ sowie das ABM-System einer Methylengruppe. Erstgenannte weisen COSY-Korrelationen zu dem zuvor beschriebenen Spinsystem auf, letztere $\mathrm{CH}_{2}$-Gruppe bildet das Ende einer Alkylkette, bestehend aus einer Methin-, einer Methyl- und drei Methylengruppen (Abbildung 35). 
Zusätzlich wird noch das Multiplett einer zweiten Methylgruppe aufgezeigt, die Kopplungskonstanten um $1.0 \mathrm{~Hz}$ deuten ${ }^{4} \mathrm{~J}$-Kopplungen über eine Doppelbindung an, auch die Verschiebung von $\delta_{\mathrm{H}}=1.71$ spricht für die Bindung an ein $\mathrm{sp}^{2}$ hybridisiertes C-Atom. Ein in deuteriertem Pyridin aufgenommenes Protonenspektrum offenbart zusätzlich drei austauschbare Wasserstoffatome.

Aus dem ${ }^{13} \mathrm{C}-\mathrm{NMR}$-Spektrum gehen 11 aliphatische Kohlenstoffatome hervor, fünf davon Sauerstoff-gebunden, sowie vier olefinische C-Atome. Signale um 100 ppm lassen auf die Anwesenheit von Acetalen oder Ketalen schließen. Die chemische Verschiebung des Signals bei $\delta_{C}=205.2$ impliziert das Vorhandensein einer Ketogruppe.

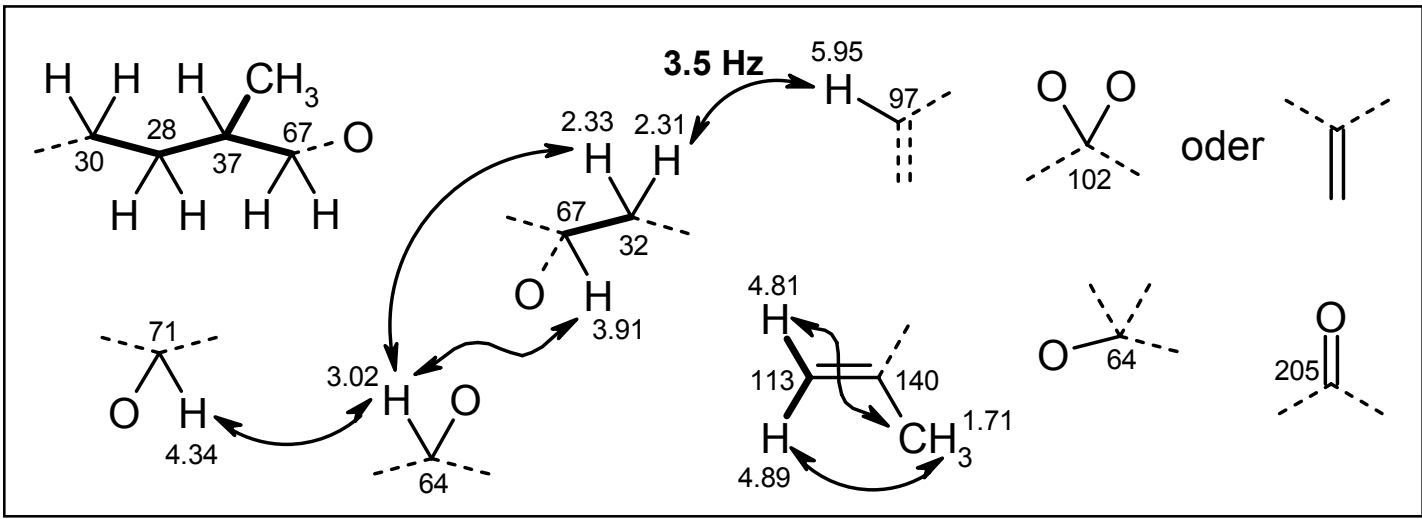

Abbildung 35: Über 1D- und 2D-NMR-Spektren ermittelte Strukturelemente von 85 mit $\mathrm{H}, \mathrm{H}$ COSY-Korrelationen. Die Verschiebungen der Atome sind in ppm angegeben.

Die Masse von $\underline{85}$ wurde mittels ESI-Massenspektrometrie zu $280 \mathrm{~g} / \mathrm{mol}$ bestimmt und die Summenformel von $\mathrm{C}_{16} \mathrm{H}_{24} \mathrm{O}_{4}$ durch Hochauflösung bestätigt.

Es war nicht möglich, die in Abbildung 35 beschriebenen Strukturelemente zu einem Molekül mit dieser Summenformel zusammenzusetzen. Auch unter dem Aspekt, dass nur vier Sauerstoff-Atome für das Molekül berechnet wurden, scheint es einen Fehler in den angenommenen Strukturmerkmalen oder in der Molmasse zu geben. Die Aufnahme eines IR-Spektrums erbrachte den Beweis, dass ein Denkfehler, die Carbonylgruppe betreffend, vorlag, da keine Absorptionsbande um $1710 \mathrm{~cm}^{-1} \mathrm{zu}$ finden war. Hingegen lag eine scharfe Bande bei $1955 \mathrm{~cm}^{-1}$, die auf das Vorhandensein einer Allengruppierung hindeutete. Diese Hypothese wurde durch die chemischen Verschiebungen von $\delta_{C}=205.2,102.0$ und $98.6 \mathrm{im}$ ${ }^{13} \mathrm{C}-\mathrm{NMR}$-Experiment bestätigt. 


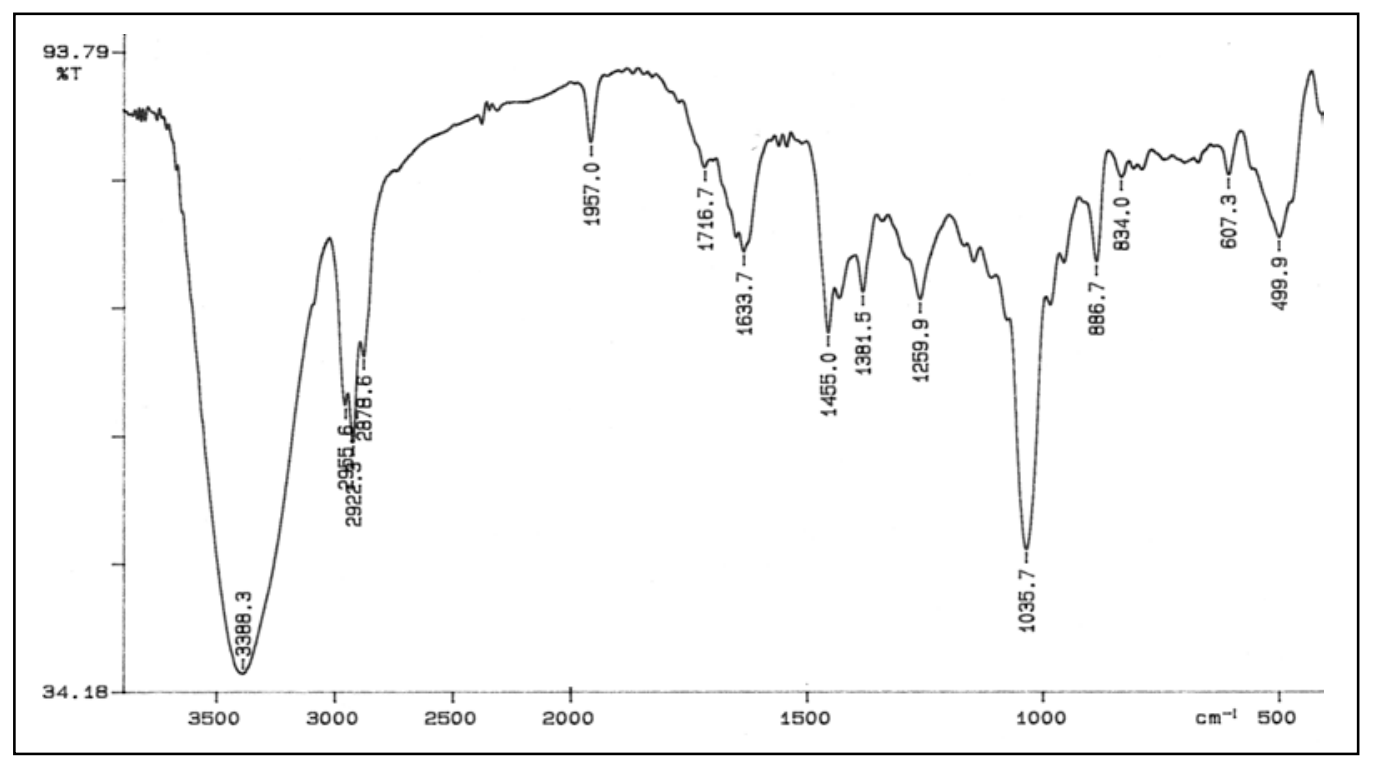

Abbildung 36: IR-Spektrum von Lupallen B (모).

Durch ein HMBC-Experiment gelingt die vollständige Verknüpfung der neu überdachten Strukturfragmente.

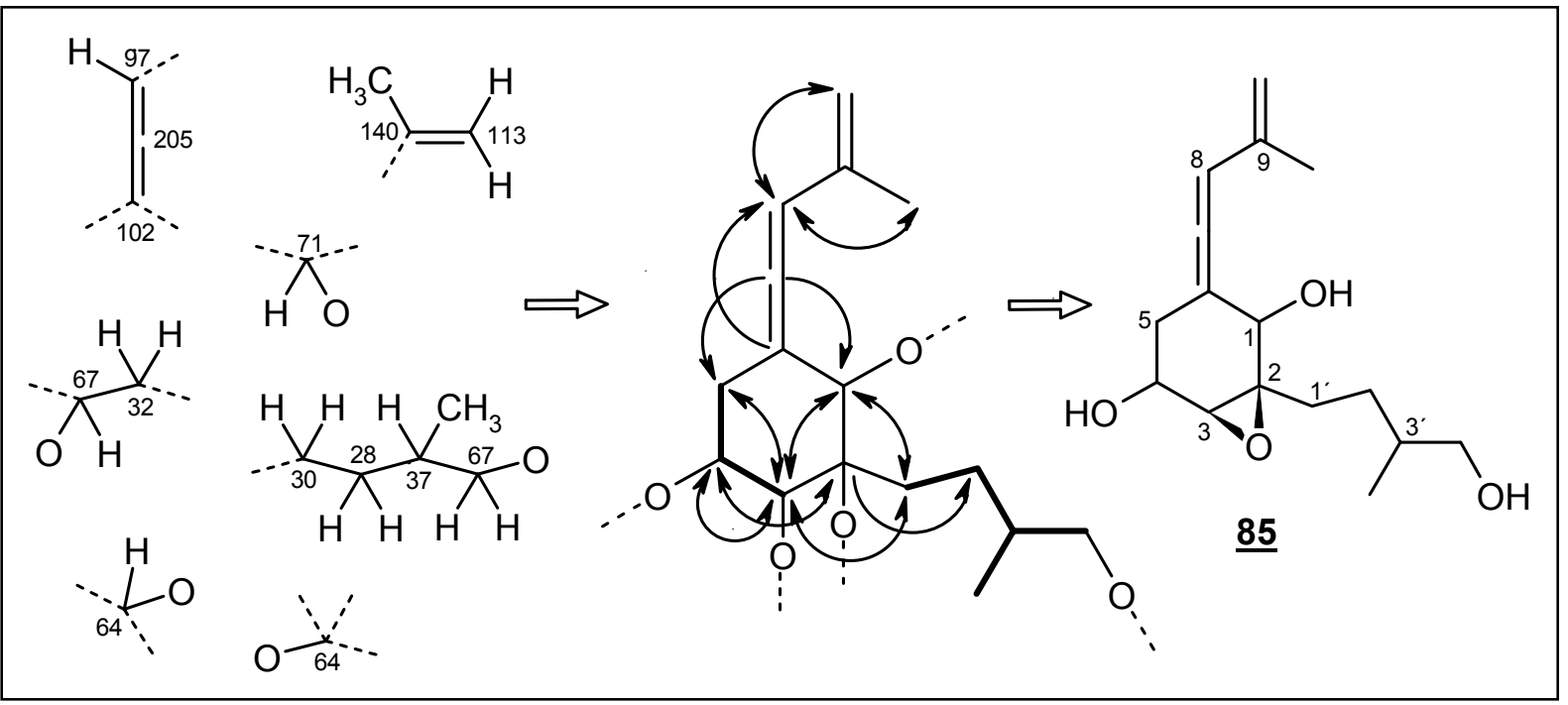

Abbildung 37: Neu überdachte Strukturteile für $\underline{85}$ und Verknüpfung der Fragmente durch $\mathrm{H}, \mathrm{H}-\mathrm{COSY}-K o r r e l a t i o n e n$ (fett) und ausgewählte HMBC-Kopplungen (Pfeile).

Aus der Summenformel errechnet sich das Vorliegen von fünf Doppelbindungsäquivalenten, von denen zwei durch das Allen, eins durch die exocyclische Doppelbindung und ein weiteres durch einen Cyclohexan-Ringschluß abgedeckt sind. Es verbleibt also noch ein Ringschluß, der aufgrund der chemischen Ver- 
schiebung der ${ }^{13} \mathrm{C}$-Signale als Epoxid zwischen C-2 und C-3 ausgemacht wurde. Die kleine Kopplung von ca. $1.0 \mathrm{~Hz}$ zwischen 3-H und 4- $\mathrm{H}$ lässt eine transpseudoaxiale Stellung für die $\mathrm{OH}-G r u p p e$ und den Oxiranring im substituierten Cyclohexan-Gerüst erwarten.

\section{Lupallen A (후)}

Das ESI-Massenspektrum von $\underline{86}$ liefert eine Molmasse von $280 \mathrm{~g} / \mathrm{mol}$. Hochauflösungen der Peaks bei $281[\mathrm{M}+\mathrm{H}]^{+}, 303[\mathrm{M}+\mathrm{Na}]^{+}, 561[2 \mathrm{M}+\mathrm{H}]^{+}$und 583 $[2 \mathrm{M}+\mathrm{Na}]^{+}$führen mit $\mathrm{C}_{16} \mathrm{H}_{24} \mathrm{O}_{4}$ zur selben Summenformel wie $\underline{\mathbf{8 5}}$. Die IR- und UVSpektren beider Verbindungen sehen ebenfalls gleich aus und auch die 1D-NMRSpektren scheinen auf den ersten Blick identisch zu sein. Bei genauer Analyse des ${ }^{1} \mathrm{H}-N M R-S p e k t r u m s$ erkennt man, dass $\underline{\mathbf{8 6}}$ nicht $100 \%$ ig sauber vorlag, sondern mit $\underline{\mathbf{8 5}}$ verunreinigt war und sich beide Substanzen nur in der chemischen Verschiebung zweier Signale $\left(8-\mathrm{H}\right.$ und $\left.11-\mathrm{H}_{3}\right)$ unterscheiden. Diese Beobachtung ließ eine axiale Isomerie bezüglich der Allengruppierung vermuten. Beim Vergleich der ${ }^{13} \mathrm{C}-\mathrm{NMR}$ Spektren (vgl. Tabelle 10) konnten bisherige Annahmen bestätigt werden, aber es traten zusätzliche Abweichungen in den chemischen Verschiebungen für C-1, C-3 und C-4 auf.

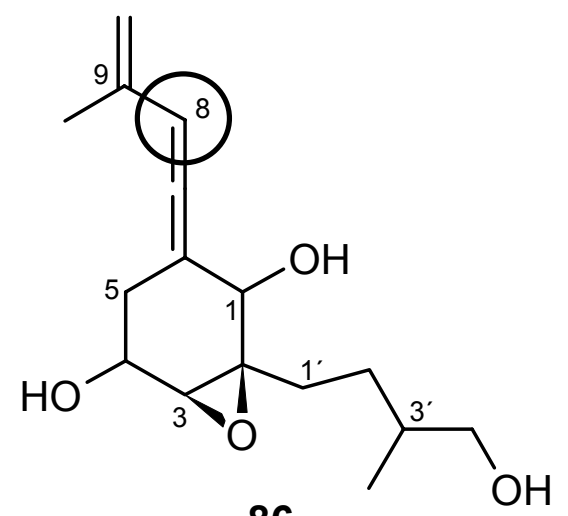

$\underline{86}$

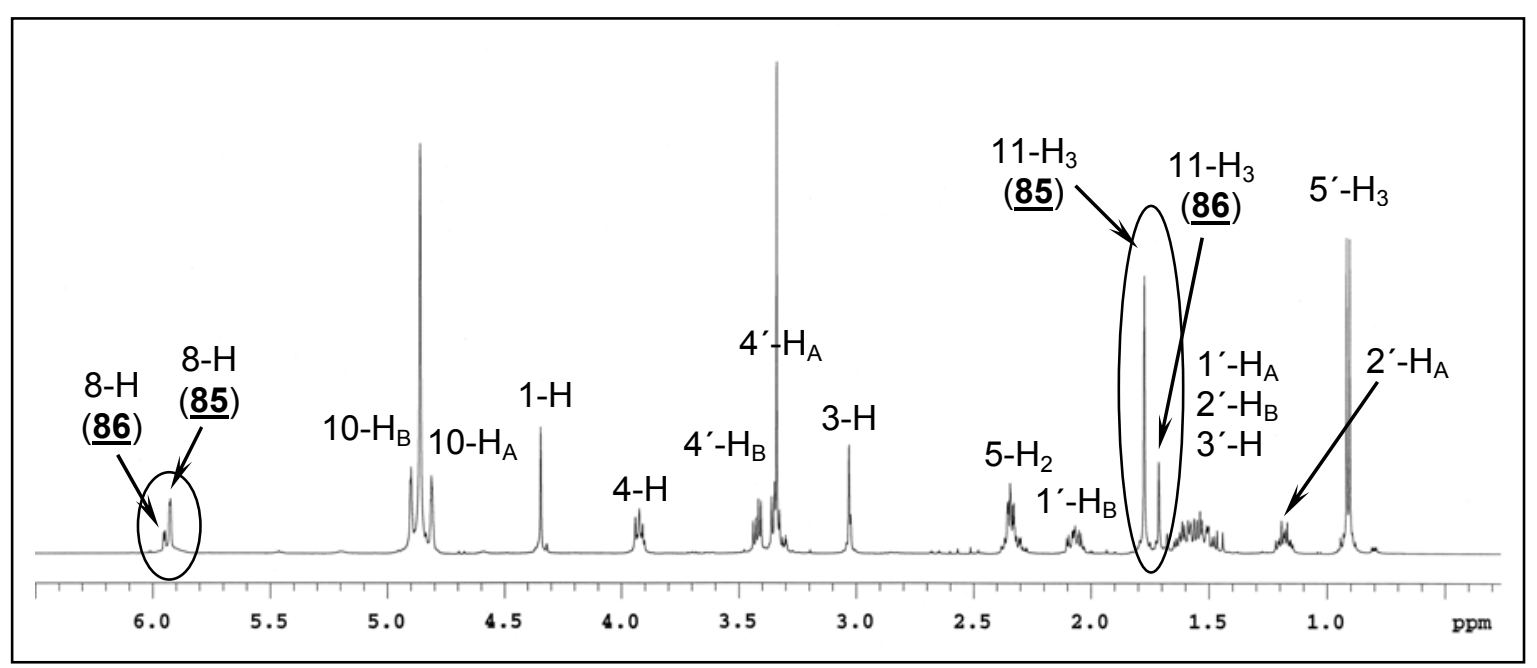

Abbildung 38: ${ }^{1} \mathrm{H}-\mathrm{NMR}-S p e k t r u m$ von $\underline{\mathbf{8 6}}$, verunreinigt mit $\underline{\mathbf{8 5}}$ (125 $\left.\mathrm{MHz}, \mathrm{CD}_{3} \mathrm{OD}\right)$. 
Anhand der NMR-Spektren erscheint es schwierig, auf die Stereochemie der Isomere zu schließen. Dieses Problem könnte durch eine Röntgenkristallstruktur gelöst werden. Es ist jedoch bis zum heutigen Zeitpunkt nicht gelungen, einen vermessbaren Kristall zu züchten.

\begin{tabular}{|c|c|c|c|c|c|c|c|}
\hline \multirow{2}{*}{\multicolumn{2}{|c|}{$\begin{array}{c}\text { Position und } \\
\text { Qualität }\end{array}$}} & \multicolumn{2}{|c|}{$\underline{86}$} & \multicolumn{2}{|c|}{$\underline{85}$} & \multicolumn{2}{|c|}{ Differenz } \\
\hline & & \multirow{2}{*}{$\begin{array}{c}{ }^{13} \mathrm{C} \\
70.27\end{array}$} & \multirow{2}{*}{$\frac{{ }^{1} \mathrm{H}}{5.00}$} & \multirow{2}{*}{$\frac{{ }^{13} \mathrm{C}}{70.52}$} & \multirow{2}{*}{$\frac{{ }^{1} \mathrm{H}}{4.96}$} & \multirow{2}{*}{$\frac{\Delta^{13} \mathrm{C}}{0.25}$} & \multirow{2}{*}{$\frac{\Delta^{1} \mathrm{H}}{0.04}$} \\
\hline 1 & $\mathrm{CH}$ & & & & & & \\
\hline 1 & $\mathrm{OH}$ & - & 7.28 & - & 7.46 & & 0.18 \\
\hline 2 & C & 64.15 & - & 64.21 & - & 0.06 & \\
\hline 3 & $\mathrm{CH}$ & 63.64 & 3.63 & 64.03 & 3.63 & 0.39 & - \\
\hline \multirow{2}{*}{4} & $\mathrm{CH}$ & 66.31 & 4.59 & 66.90 & 4.54 & 0.59 & 0.05 \\
\hline & $\mathrm{OH}$ & - & 7.15 & - & 7.15 & & - \\
\hline 5 & $\mathrm{CH}_{2}$ & 32.12 & $\begin{array}{l}2.87 \\
3.09\end{array}$ & 32.25 & $\begin{array}{l}2.82 \\
3.08\end{array}$ & 0.13 & $\begin{array}{l}0.05 \\
0.01\end{array}$ \\
\hline 6 & C & 103.21 & - & 103.05 & - & 0.16 & \\
\hline 7 & C & 204.03 & - & 204.18 & - & 0.15 & \\
\hline 8 & $\mathrm{CH}$ & 97.94 & 6.15 & 97.53 & 6.09 & 0.41 & 0.06 \\
\hline 9 & C & 140.04 & - & 140.17 & - & 0.13 & \\
\hline 10 & $\mathrm{CH}_{2}$ & 113.64 & $\begin{array}{l}4.85 \\
5.01\end{array}$ & 113.56 & $\begin{array}{l}4.90 \\
5.00\end{array}$ & 0.09 & $\begin{array}{l}0.05 \\
0.01\end{array}$ \\
\hline 11 & $\mathrm{CH}_{3}$ & 19.71 & 1.83 & 20.08 & 1.95 & 0.37 & 0.12 \\
\hline $1^{\prime}$ & $\mathrm{CH}_{2}$ & 30.45 & $\begin{array}{l}1.91 \\
2.51 \\
\end{array}$ & 30.41 & $\begin{array}{l}1.94 \\
2.51 \\
\end{array}$ & 0.04 & $\begin{array}{c}0.03 \\
-\end{array}$ \\
\hline $2^{\prime}$ & $\mathrm{CH}_{2}$ & 27.76 & $\begin{array}{l}1.55 \\
2.08\end{array}$ & 27.69 & $\begin{array}{l}1.53 \\
2.08\end{array}$ & 0.07 & $\begin{array}{c}0.02 \\
-\end{array}$ \\
\hline $3^{\prime}$ & $\mathrm{CH}$ & 36.68 & 1.82 & 36.77 & 1.82 & 0.09 & - \\
\hline \multirow[t]{2}{*}{$4^{\prime}$} & $\mathrm{CH}_{2}$ & 67.27 & $\begin{array}{l}3.66 \\
3.73\end{array}$ & 67.33 & $\begin{array}{l}3.66 \\
3.73\end{array}$ & 0.06 & $\begin{array}{l}- \\
-\end{array}$ \\
\hline & $\mathrm{OH}$ & - & 5.84 & - & 5.83 & & 0.01 \\
\hline $5^{\prime}$ & $\mathrm{CH}_{3}$ & 17.17 & 1.04 & 17.19 & 1.04 & 0.02 & - \\
\hline
\end{tabular}

Tabelle 10: Vergleich NMR-Daten von 85 und 86 .

Die Substanzen wurden in $\overline{\mathrm{D}_{5}}$-Pyridin bei $35^{\circ} \mathrm{C}$ mit $125 \mathrm{MHz}$ gemessen. 


\section{Lupallen C (호)}

Da das farblose Öl das gleiche Anfärbeverhalten wie $\underline{85}$ und $\underline{86}$ aufweist, war eine verwandte Struktur zu erwarten. Die Aufnahme eines IR-Spektrums bestätigte das Vorliegen einer Allengruppierung $\left(1956 \mathrm{~cm}^{-1}\right)$, des Weiteren erscheint eine Absorptionsbande bei $1718 \mathrm{~cm}^{-1}$, charakteristisch für aliphatische Ester. Aus den zusätzlichen Signalen im ${ }^{1} \mathrm{H}$ - und ${ }^{13} \mathrm{C}-\mathrm{NMR}$-Spektrum $\left(\delta_{\mathrm{H}}=1.97, \delta_{\mathrm{C}}=21.0\right.$ und 170.3) geht hervor, dass $\underline{85}$ um eine Acetoxyfunktion erweitert wurde. Bestätigt durch eine Tieffeldverschiebung von $\delta_{\mathrm{H}}=4.54$ nach 6.00 für $4-\mathrm{H}$ ist die Acetateinheit durch 4-OH mit dem restlichen Molekül verknüpft. Ein Vergleich der Protonenspektren von $\underline{87}$ und $\underline{85}$ zeigt an, dass einige Signale von Lupallen $\mathrm{C} z \mathrm{u}$ erheblich tieferem Feld verschoben sind, genannt seien hier $3-\mathrm{H}\left(\delta_{\mathrm{H}}=4.48\right.$ statt 3.63), $1^{\prime}-\mathrm{H}\left(\delta_{\mathrm{H}}=2.44\right.$ statt 1.94) und das axiale Proton der Methylengruppe an Position $5\left(\delta_{\mathrm{H}}=3.40\right.$ statt 3.08$)$. Noch drastischer sind die Unterschiede im ${ }^{13} \mathrm{C}$ NMR-Spektrum, besonders für die Resonanzen der Cyclohexan-Kohlenstoffatome. Für C-2 und C-3 beispielsweise gehen die chemischen Verschiebungen um 10 ppm auseinander.

Mit einer Molmasse von $340 \mathrm{~g} / \mathrm{mol}$ konnte durch ESI-Hochauflösung eine Summenformel von $\mathrm{C}_{18} \mathrm{H}_{28} \mathrm{O}_{6}$ berechnet werden. Die Massendifferenz von 60 Einheiten zu $\underline{\mathbf{8 5}}$ ergibt sich zum einen aus der Acetylierung der 4-Hydroxygruppe, zum anderen durch die Addition von Wasser. Letzteres verlangt die Öffnung des Epoxids, was mit einer Entspannung des Ringsystems einher geht und folglich mit Resonanzverschiebungen zu tieferem Feld.

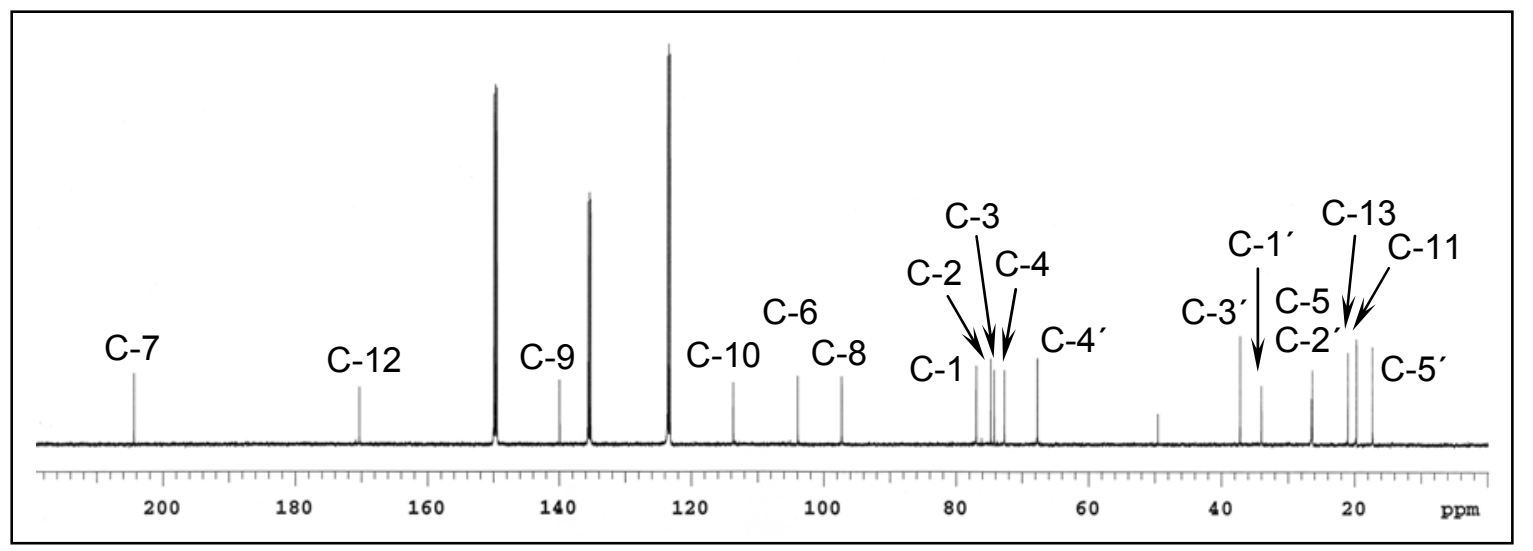

Abbildung 39: ${ }^{13} \mathrm{C}-\mathrm{NMR}-S p e k t r u m$ von Lupallen $\mathrm{C}(\underline{\mathbf{8 7}})\left(125 \mathrm{MHz}, \mathrm{D}_{5}\right.$-Pyridin, $\left.35^{\circ} \mathrm{C}\right)$. 
Durch Betrachtung der Kopplungskonstanten und unter Zuhilfenahme eines NOESY-NMR-Spektrums können einige Aussagen zur relativen Stereochemie gemacht werden. Die Kopplungskonstanten von $J=12.0,5.0$ und $2.5 \mathrm{~Hz} z$ wischen dem Signal zugehörig zum Proton geminal zur Acetoxygruppe (4-H) und dem ABMX-Spinsystem der Methylengruppe an C-5 sowie zum ehemaligen OxiranProton sind charakteristisch für eine axial-axial- und zwei äquatorial-axialKopplungen. Da C-2 nicht Protonen-tragend ist, erhält man keine Hinweise über die Stereochemie an diesem Zentrum. Unter Berücksichtigung sterischer Effekte kann aber davon ausgegangen werden, dass die Seitenkette eine äquatoriale Position einnimmt. Die fehlende NOESY-Korrelation zwischen dem axialen Wasserstoffatom an C-5 und dem Signal bei $\delta_{H}=4.57$ deutet auf eine fehlende räumliche Nähe beider Protonen und somit auf eine äquatoriale Stellung für $1-\mathrm{H}$ hin. Die räumliche Nachbarschaft zu jeweils einem Proton der Methylengruppen an C-1'und C-2' stehen in Einklang mit diesem Vorschlag.

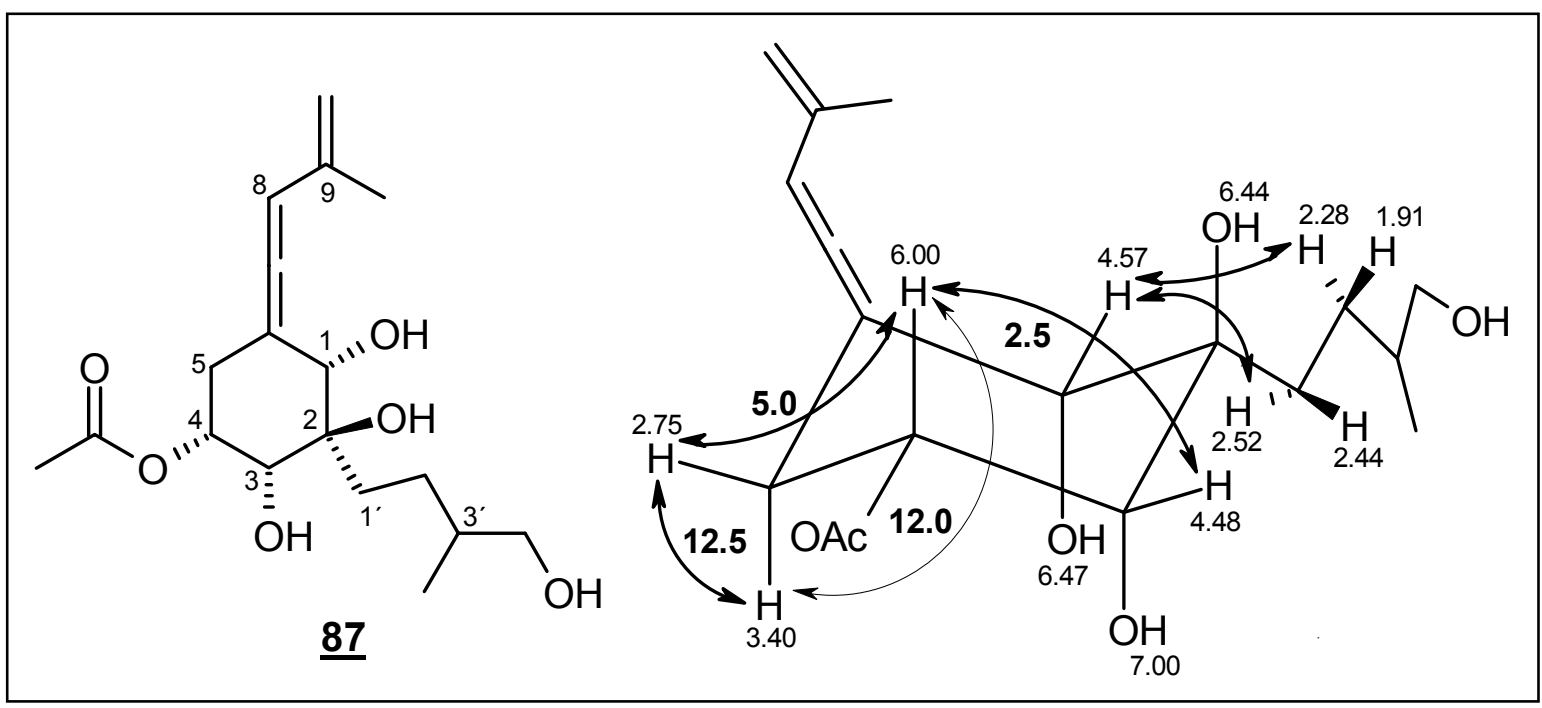

Abbildung 40: Herleitung der relativen Stereochemie von Lupallen $\mathrm{C}(\underline{\mathbf{8 7}})$ durch ${ }^{1} \mathrm{H}$-Kopplungskonstanten (fette Zahlen) und NOESY-Korrelationen (dicke Pfeile); chemische Verschiebungen in ppm.

Die isolierten Allene sind als Naturstoffe so bisher nicht beschrieben. Eine Datenbankrecherche in $\underline{\text { Antibase }}^{47}$ führte zu zwei ähnlichen Pilzmetaboliten, den exocyclischen Allenen $\underline{\mathbf{8 8}}^{132}$ und $\underline{\mathbf{8 9}}^{133} \underline{\mathbf{8 8}}$ wurde aus dem Schlauchpilz Eutypa lata isoliert, der Obstbäume und Reben befällt und durch die verursachte Eutypiose zum Absterben der Gewächse führt. 


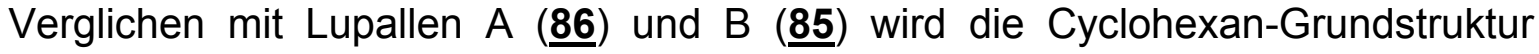
durch die gleiche Allenseitenkette ergänzt. Die unterschiedliche Substitution an C-2 läßt eine Biosynthese über die selbe Route vermuten, die lediglich in den späten Biosyntheseschritten varriert.

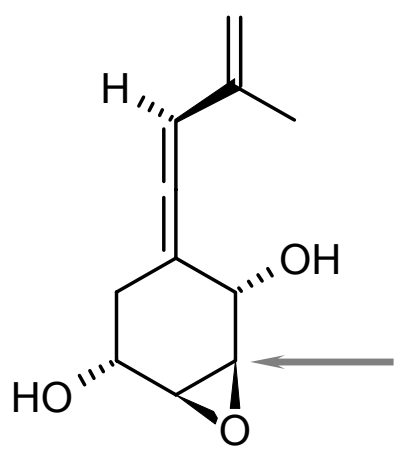

$\underline{88}$

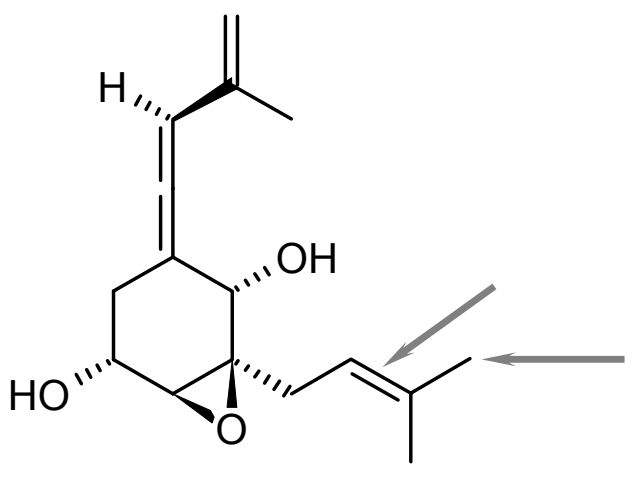

$\underline{89}$

\subsection{Variation der Kultivierungsbedingungen}

Da die Metabolite neu sind und zudem bislang wenig über Allen-Biosynthesen bekannt ist, erschien es interessant, sich mit der Biogenese der Lupallene zu beschäftigen. Zunächst sollte versucht werden, durch Variation der Kultivierungsbedingungen die Ausbeute der Substanzen zu erhöhen. Zusätzlich war zu prüfen, inwieweit dadurch eine Veränderung im Metabolitenmuster des Stammes WDMH46 erreicht werden konnte. Diese im Arbeitskreis von A. ZEECK entwickelte Verfahrensweise wird als OSMAC-Methode (one-strain-many-compounds) bezeichnet und zielt darauf ab, den Stamm verschiedenen Stresssituationen auszusetzen wie z.B. veränderten Kultivierungsbedingungen oder dem Zusatz von Enzyminhibitoren, um so neue Soffwechselleistungen im Organismus zu induzieren. Dieser Ansatz konnte erfolgreich an mehreren Streptomyceten- und Pilzstämmen angewendet werden. So ließen sich aus dem Stamm Gö 40/10 17 Metabolite aus vier verschiedenen Strukturklassen isolieren. ${ }^{134}$ Einige Beispiele für Parameter, die sich im Labor leicht variieren lassen, sind:

$$
\begin{array}{ll}
\text { - Nährmedium } & \text { - Art des Kultivierungsgefäßes } \\
\text { - Fermentationszusätze } & \text { - Belüftung } \\
\text { - Temperatur } & \text { - Belichtung } \\
\text { - pH-Wert. } &
\end{array}
$$


Für WDMH46 wurde hauptsächlich der Einfluß von Fermentationszusätzen sowie der Kultivierungsgefäße und damit der Belüftung untersucht. Die Aufarbeitung der Kulturen wurde wie unter Kapitel 3.2. beschrieben durchgeführt, die Analyse des Metabolitenmusters erfolgte durch Dünnschichtchromatographie.

Da es sich bei WDMH46 um ein Isolat aus einem marinen Habitat handelt, schien der Salzgehalt in der Kulturlösung ein interessanter Aspekt zu sein. Deshalb wurde der Stamm im Nährmedium 1158, bei dem zuvor eine NaCl-Konzentration von 0.2, 1, 2 und 4\% eingestellt worden war, kultiviert. Die Bedeutung von Salzen als Nährmedienbestandteil sei kurz an zwei Beispielen erläutert. Bei Streptomyces griseoflavus (W 384) bewirkt die Zugabe von $25 \mathrm{~g} / \mathrm{L} \mathrm{NaCl}$ eine Ausbeutesteigerung des Peptidlactons Hormaomycin, ${ }^{66}$ bei Streptomyces cellulosae (S 1013) hat der Zusatz von $1 \mathrm{~g} / \mathrm{L}$ Natriumbromid einen neuen Metaboliten (Hexacyclinsäure) hervorgebracht. ${ }^{135}$ Die Kulturen von WDMH46 mit und ohne Salzzusatz wiesen optisch keine Unterschiede auf. In den Extrakten der salzhaltigen Kulturen konnten allerdings keine Allene nachgewiesen werden, woraus sich schlussfolgern lässt, dass $\mathrm{NaCl}$ zwar vom Organismus vertragen wird, aber die Allenproduktion inhibiert.

Auch der Zusatz von Oberflächenmaterialien wie XAD-2 hat sich schon in früheren Arbeiten für die Erweiterung der Produktpalette oder zur Ausbeutesteigerung verschiedener Metabolite bewährt. So konnte C. PUDER in seiner Dissertation zeigen, dass die Beigabe von Celite zu einem Streptomyceten-Stamm zu einer Vervierfachung der Rubromycin-Ausbeute führt, der Zusatz von Erde bewirkte die Bildung von drei neuen Metaboliten. ${ }^{136}$ Erklärungsversuche deuten die Anwesenheit dieser Materialien als Stressfaktor. Denkbar ist auch eine Beeinflussung der feedback-Regulation der Biosynthese durch Adsorbtion der Metabolite oder Substrate, ${ }^{137}$ ebenso wie die Theorie, dass durch Anlagerung einzelner Zellen an die Oberflächenmaterialien deren Wachstum gesteigert wird.

Die Zugabe des Adsorberharzes Amberlite-XAD-2 (3 g/L) zum Allenproduzenten brachte nicht die erhoffte Ausbeutesteigerung, im Gegenteil: die Myzelbildung kam zum Erliegen und die mit Anis pink färbenden Metabolite waren im KultufiltratExtrakt nur noch in Spuren zu detektieren, im XAD-Eluat überhaupt nicht.

Der Sauerstoffpartialdruck in einer Kulturlösung kann sich stark auf das Metabolitenspektrum eines Stammes auswirken. ${ }^{138}$ Die Wahl des Kultivierungsgefäßes bestimmt erheblich die Durchmischung der Kultur und damit die Belüftung. Im 
Fermenter ist sie beispielsweise abhängig von der Art und Anzahl der Rührblätter und der Rührergeschwindigkeit. In Schüttelkolben entstehen Belüftungsunterschiede vor allem durch die Verwendung von Kolben mit oder ohne Schikanen, sowie durch die Art des Verschlusses und der Umdrehungszahl des Schüttlers. Besonders drastische Änderungen des Sauerstoffgehaltes sind im im AirliftFermenter möglich, der bei bis zu 5 bar Überdruck betrieben werden kann. Als anderes Extrem sei die Ruhefermtation im P-Kolben genannt, bei der keine Durchmischung der Kulturbrühe erfolgt.

Zunächst wurden mit dem Stamm Ruhefermentationen durchgeführt. Um den Zusammenhang zwischen Nährstoffangebot und Metabolitenproduktion nochmals zu kontrollieren, geschah dies in sieben Nährmedien (1158, E2, G20, 1405, 1549, Marine Broth und Biomalz). Die Kultivierung erfolgte sowohl als Flüssigkultur in PKolben $(1 \mathrm{~L})$ und Erlenmeyerkolben $(500 \mathrm{~mL})$ als auch auf Agar $(500 \mathrm{~mL})$, parallel lief eine Versuchsreihe mit 3 \%igen $\mathrm{NaCl}-\mathrm{Nährlösungen} \mathrm{mit.} \mathrm{Bei} \mathrm{allen} \mathrm{Versuchen}$ mit mangelnder Belüftung ging die Sekundärmetaboliten-Produktion drastisch zurück, was sicherlich auf das schlechte Wachstum des Stammes zurückzuführen ist. Während in 1158 und E2 die pinkfarbenen Zonen auf den DC-Platten noch halbwegs gut zu sehen waren, konnte man sie in den anderen Medien nur mehr erahnen, bei den Extrakten mit $\mathrm{NaCl}$-Zusatz konnte kein Sekundärmetabolit nachgewiesen werden. Eine Fermentation unter Sauerstoffmangel ist für den Stamm WDMH46 offensichtlich ebenso wenig geeignet wie der Zusatz von Natriumchlorid.

Aufgrund dieser Ergebnisse kann man vermuten, dass sich die Biosyntheseleistung des Pilzstammes durch ein erhöhtes Sauerstoffangebot steigern lässt.

Zur Überprüfung dieser Hypothese wurde der Stamm WDMH46 im Biostat BFermenter zeitgleich in 2 und 5 Litern 1158 kultiviert. Nach dem Animpfen mit drei Tage alten Vorkulturen wurde eine Rührergeschwindigkeit von $500 \mathrm{rpm}$ und eine Luftzufuhr von 1.0 vvm eingestellt und der Fermentationsverlauf durch Kontrolle von $\mathrm{pH}$-Wert und Sauerstoffgehalt verfolgt. Beim Vergleich beider Ansätze am nächsten Tag war im 5 L-Gefäß deutlich mehr Zellmasse zu verzeichnen und die Kultur war wesentlich dunkler gefärbt. Als Ursache wurde das Absinken des Sauerstoffgehaltes im 2 L-Gefäß auf $8.4 \%$ angenommen und die Luftzufuhr auf 2 vvm nachreguliert. Zur Ernte nach sieben Tage hatte sich die Kultur im 5 LGefäß dunkelbraun gefärbt, die 2 L-Lösung war beige, wies einen stechenden 
Geruch ähnlich Buttersäure auf und wurde verworfen. Das Metabolitenmuster des 5 L-Ansatz war mit dem aus Schüttelkolben vergleichbar, die Ausbeute sogar erhöht. Zusätzlich traten zwei neue Zonen im Anis-besprühten Dünnschichtchromatogramm auf: eine mit bläulicher Farbe, die andere mit dem pinken Farbton der Allene, aber einem $R_{\mathrm{f}}$-Wert polarer als die bislang gefundenen. Die Substanzen wurden später als $\underline{97}$ und $\underline{91}$ identifiziert. Die gleichen Ergebnisse wurden mit einer Fermentation im 10 L-Biostat-E-Fermenter erzielt.

Da der Allenproduzent in seiner Biosyntheseleistung offensichtlich durch einen hohen Sauerstoffpartialdruck gepuscht wird, sollte sich als nächster Schritt eine Überdruckfermentation im Airlifter anschließen. Im Airlift-Fermenter erfolgt im Unterschied zum Rührblattfermenter die Durchmischung der Kulturlösung durch das Einleiten von Luft vom Boden des Kultivierungsgefäßes aus. Zusätzlich kann der Gesamtdruck im Fermenter über ein Absperrventil hinter dem Abluftfilter reguliert werden. So sind Fermentationen sowohl unter Normaldruck wie auch bei bis zu 5 bar Überdruck möglich.

In den Vorversuchen hatte sich gezeigt, dass das Pilzwachstum und die Produktion der Allene im Nährmedium 1158 am besten war. Allerdings hatte sich schon während der Fermentation im 10 L-Biostat E-Fermenter angedeutet, dass die starke Myzelbildung die Messfühler für überwachbare Parameter beeinträchtigte und sich so negativ auf die Kontrolle der Fermentation auswirkte. Aus diesem Grund wurde von einer Kultivierung in 1158 Abstand genommen und im Hinblick auf eine erleichterte Aufarbeitung und Substanzisolierung die Nutzung einer synthetischen Nährlösung ins Auge gefasst. Um dennoch ein gutes Anwachsen des Organismus zu gewährleisten, wurde das Medium 1158 für die Vorkulturen beibehalten. Dementsprechend wurden $9.5 \mathrm{~L} \mathrm{NL} 1405$ vorgelegt und mit vier Tage alten Vorkulturen, die in 1 L-Schüttelkolben (170 mL NL 1158) bei $28{ }^{\circ} \mathrm{C}$ und 120 spm angezogen wurden, angeimpft.

Erfahrungsgemäß verläuft die Sekundärmetabolitenproduktion im Airlift-Fermenter zeitlich schneller als in Schüttelkultur oder den Biostat-Rührblatt-Fermentern. Deshalb wurden zweimal täglich Tüpfelproben von $20 \mathrm{~mL}$ entnommen und die Sekundärmetaboliten-Produktion dünnschichtchromatographisch verfolgt. Schon nach zwei Tagen war der Entnahmeschlauch verstopft, nach einem weiteren Tag hatten Teile der Kultur das Gefäß verlassen. Daraufhin wurde die Fermentation abgebrochen und ein erneuter Versuch so verändert, dass sowohl für die Haupt- 
kultur als auch für die Vorkultur das synthetische Nährmedium 1405 verwendet wurde. Bei der Untersuchung der Fermentationsproben wurde beobachtet, dass der Fermentationsverlauf von Schüttelkolben nicht auf den Airlift-Fermenter übertragbar war. Als nach fünf Tagen die Allenproduktion, sichtbar durch die pinke Farbbildung mit Anis, zurückging, wurde die Inkubation beendet und die Kulturbrühe geerntet. Die dünnschichtchromatographische Analyse der Extrakte legte ein erweitertes Metabolitenmuster dar. Die Allene $\underline{\mathbf{8 6}}, \underline{\mathbf{8 7}}$ und $\underline{\mathbf{9 1}}$ wurden in guten Ausbeuten gebildet. Es waren aber noch mehrere Zonen mit $\mathrm{R}_{\mathrm{f}}$-Werten von 0.3 - 0.4 zu erkennen, deren Strukturen zu $\underline{94}$ und $\underline{96}$ bestimmt wurden. Bei einer Inkubation des Stammes WDMH46 im Airlifter mit 5 bar Überdruck wurde keine Produktion der Allene beobachtet, auf eine weitere Aufreinigung des Ansatzes wurde verzichtet.

Die Ausbeute der Allene konnte im Fermenter zwar gesteigert werden, wegen des unregelmäßigen Fermentationsverlaufes erschienen Schüttelkolben im Hinblick auf Fütterungsexperimente dennoch die bessere Wahl.

Liegt die biosynthetische Herkunft einer Struktur im Dunkeln, beginnen Biogeneseexperimente meist mit der Zufütterung von Acetat, dem Baustein der Polyketide. Um die Verträglichkeit des Allenbildners auf dieses Substrat zu testen, wurde der Stamm WDMH46 in den Nährlösungen 1158 und 1405 zuzüglich 1 g/L Natriumacetat über eine Dauer von vier, fünf bzw. sechs Tagen inkubiert. So können spezifische Aussagen über den Verlauf der Sekundärmetaboliten-Produktion im jeweiligen Medium gemacht werden. Schon in den viertägigen Kulturen beider Nährlösungen war eine Allenproduktion zu verzeichnen, die bis zum sechsten Tag noch gesteigert wurde. Dabei machte es keinen Unterschied, ob Erlenmeyerkolben mit oder ohne Schikanen verwendet wurden. In den Dünnschichtchromatogrammen konnte die breite Zone mit einem $\mathrm{R}_{\mathrm{f}}$-Wert um $0.3\left(\mathrm{CH}_{3} \mathrm{Cl} / \mathrm{MeOH}\right.$ 9:1) den

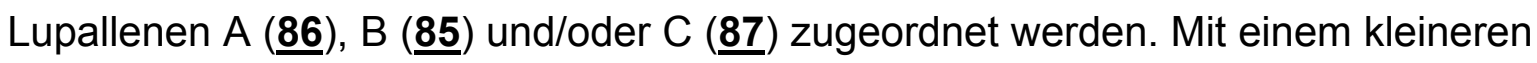
$\mathrm{R}_{\mathrm{f}}$-Wert von 0.1 wurde auch die Anwesenheit von $\underline{91}$ bestätigt, ebenso wie die eines neuen Metaboliten $\underline{\mathbf{9 0}}$, der wegen seiner Anfärbung mit Anis nach pink und dem $R_{f}$-Wert von 0.5 als lipophileres Allen eingestuft wurde. Obwohl im 1158Extrakt die Intensität der pinkfarbenen DC-Zonen deutlich höher war als im 1405Extrakt, waren die isolierten Mengen der Metabolite $\underline{86}, \underline{87}, \underline{90}$ und $\underline{91}$ etwa gleich. Die Ausbeuten lagen um $5 \mathrm{mg} / \mathrm{L}$. Da sich die Aufreinigung der Metabolite aus 
1405-Kulturen wesentlich unkomplizierter bewerkstelligen ließ, wurde 1405 als Nährlösung für Biogenese-Untersuchungen ausgewählt.

Die Ergebnisse der Fermentationsvariationen sind in nachfolgendender Tabelle 11 zusammengefasst. Neben den hier beschriebenen Verbindungen konnten in den HPLC-Chromatogrammen (HPLC 1, Säule 2, Programm 3) der verschiedenen Trennschritte weitere Sekundärmetabolite nachgewiesen werden, deren Aufreinigung wegen geringer Substanzmengen nicht erfolgreich war.

\begin{tabular}{|c|c|c|}
\hline Kultivierungsgefäß & $\underline{85}, \underline{86} \& \underline{87}$ & neue Metabolite \\
\hline \multicolumn{3}{|l|}{ Schüttelkulturen: } \\
\hline mit 3g/L XAD-2-Zusatz & \pm & - \\
\hline $1158+1 \mathrm{~g} / \mathrm{L} \mathrm{NaOAc}$ (o.S.) & ++ & $\underline{90}$ \\
\hline $1158+1 \mathrm{~g} / \mathrm{L} \mathrm{NaOAc}$ (m.S.) & ++ & $\underline{90}$ \\
\hline $1405+1 \mathrm{~g} / \mathrm{L} \mathrm{NaOAc}$ (o.S.) & ++ & $\underline{90}$ \\
\hline $1405+1 \mathrm{~g} / \mathrm{L} \mathrm{NaOAc}$ (m.S.) & ++ & $\underline{90}$ \\
\hline \multicolumn{3}{|l|}{ Ruhekulturen: } \\
\hline P-Kolben & $\pm /+(1158, \mathrm{E} 2)$ & - \\
\hline P-Kolben mit 3\% NaCl & - & - \\
\hline SK & $\pm /+(1158, E 2)$ & - \\
\hline SK mit $3 \% \mathrm{NaCl}$ & - & - \\
\hline Agarkultur & $\pm /+(1158, E 2)$ & - \\
\hline Agarkultur mit $3 \% \mathrm{NaCl}$ & - & - \\
\hline \multicolumn{3}{|l|}{ Fermenter: } \\
\hline 2 L-Biostat B & - & - \\
\hline 5 L-Biostat B & +++ & $\underline{91}, \underline{92}$ \\
\hline 10 L-Biostat E & +++ & 了 $\underline{93}, \underline{95}, \underline{97}$ \\
\hline 10 L-Airlifter (Normaldruck) & ++ & $\underline{91}, \underline{94}, \underline{96}$ \\
\hline 10 L-Airlifter (5 bar) & - & - \\
\hline
\end{tabular}

Tabelle 11: Metabolitenprofil vom Stamm WDMH46 unter veränderten Kultivierungsbedingungen; o./m.S.: ohne/mit Salzzusatz, SK: Schüttelkolben;

$+++/++/+$ - sehr gute/gute/mäßige/keine Produktion, \pm Produktion in Spuren. 


\subsection{Isolierung und Charakterisierung der neuen Metabolite}

Lupallen D (푸) $(7.9 \mathrm{mg})$, das der Stamm WDMH46 in Schüttelkolben mit Acetatzusatz gebildet hat, wurde durch Säulenchromatographie an Kieselgel $\left(\mathrm{CH}_{2} \mathrm{Cl}_{2} /\right.$ $\mathrm{MeOH}$ 15:1) und Sephadex LH-20 (Aceton) aus dem Kulturfiltrat-Extrakt erhalten. Dieses erscheint auf dem DC einheitlich, zeigte aber in der HPLC-MS-Analyse zwei Substanzpeaks mit gleicher Masse im Verhälnis 3:2, die sich nicht weiter auftrennen ließen.

Aus den vereinigten Extrakten, gebildet aus dem Kulturfiltrat der 5 L- und $10 \mathrm{~L}$ Biostat Fermenter, konnten analog zum Schema $6.1188 .0 \mathrm{mg}$ Lupallen A (흐) und $239.2 \mathrm{mg}$ Lupallen C (푸) gewonnen werden. Nach dem ersten Chromatographieschritt an Kieselgelsäule $\left(\mathrm{CH}_{2} \mathrm{Cl}_{2} / \mathrm{MeOH}\right.$ 15:1) lag der neu aufgetretene Metabolit $\underline{91}$ mit einem $\mathrm{R}_{\mathrm{f}}$-Wert um 0.1 angereichert vor und wurde mittels Sephadex $\mathrm{LH}-20(\mathrm{MeOH})$ von den restlichen Verunreinigungen befreit. Die mit Anis blau anfärbende Verbindung wurde der gleichen Prozedur unterworfen. Eine Reinheitskontrolle an der HPLC war positiv und zeigt den Substanzpeak für $\underline{97}$ bei einer Retentionszeit von 29.4 min.

Da der Stamm eine Vielzahl an Metaboliten produziert, die einen $\mathrm{R}_{\mathrm{f}}$-Bereich von 0.2 bis 0.4 aufweisen und mit Anis pink anfärben, wurde dazu übergegangen, die einzelnen Fraktionen durch HPLC zu analysieren (HPLC 1, Programm 3, Säule 2). So konnten den bislang aufgereinigten Sekundärmetaboliten folgende Retentionszeiten zugeordnet werden:

\begin{tabular}{|c|c|}
\hline Substanz & Retentionszeit \\
\hline Lupallen A (후) & 17.9 \\
\hline Lupallen B (호) & 18.9 \\
\hline Lupallen C (주) & 17.6 \\
\hline$\underline{90}$ & 29.3 \\
\hline$\underline{91}$ & 9.3 \\
\hline$\underline{97}$ & 29.4 \\
\hline
\end{tabular}

Tabelle 12: Retentionszeiten der bislang isolierten Metabolite aus WDMH46 
Des Weiteren gab es einige HPLC-Fraktionen mit einer Retentionszeit von 22.1 min. Diese wurden vereinigt und über die präparative HPLC (HPLC 3, Säule 5, Programm 6) getrennt. Der Auftrennungsschritt ergab drei Reinsubstanzen als farbloses Öl: $1.3 \mathrm{mg} \underline{92}, 0.9 \mathrm{mg} \underline{93}$ und $1.1 \mathrm{mg} \underline{95}$.

Das Kulturfiltat aus dem 10 L-Airlift-Fermenter wurde an Kieselgel $\left(\mathrm{CH}_{2} \mathrm{Cl}_{2} / \mathrm{MeOH}\right.$ 15:1) chromatographiert. Weitere Aufreinigung der 5. Rohprodukt-Fraktion an

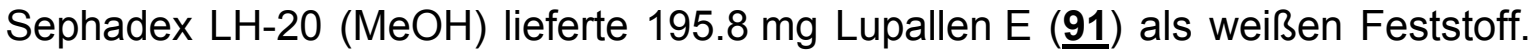
Die 2. und 3. Fraktion der Rohprodukt-Säule wurden durch Sephadex LH-20 (Aceton) von Verunreinigungen befreit. Man erhielt $12.7 \mathrm{mg} \underline{94}$ und $36.8 \mathrm{mg} \underline{96}$ in Form eines farblosen Öles.

\section{Lupallen D (무)}

Die farblose Substanz färbt wie alle bislang isolierten Lupallene mit Anis pink an, aufgrund dessen eine Allenstruktur vermutet wurde. Diese Annahme wurde im IRSpektrum durch die charakteristische Absorbtionsbande bei $1957 \mathrm{~cm}^{-1}$ bestätigt, ebenso wie das Vorliegen einer Ester-Carboxylgruppe $\left(1730 \mathrm{~cm}^{-1}\right)$, die mit Hilfe des ${ }^{13} \mathrm{C}-N M R-S p e k t r u m s$ als Acetoxyfunktion identifiziert werden konnte. Sowohl im ${ }^{13} \mathrm{C}$ - wie auch im ${ }^{1} \mathrm{H}-\mathrm{NMR}$-Spektrum treten die Resonanzen als doppelter Signalsatz auf.

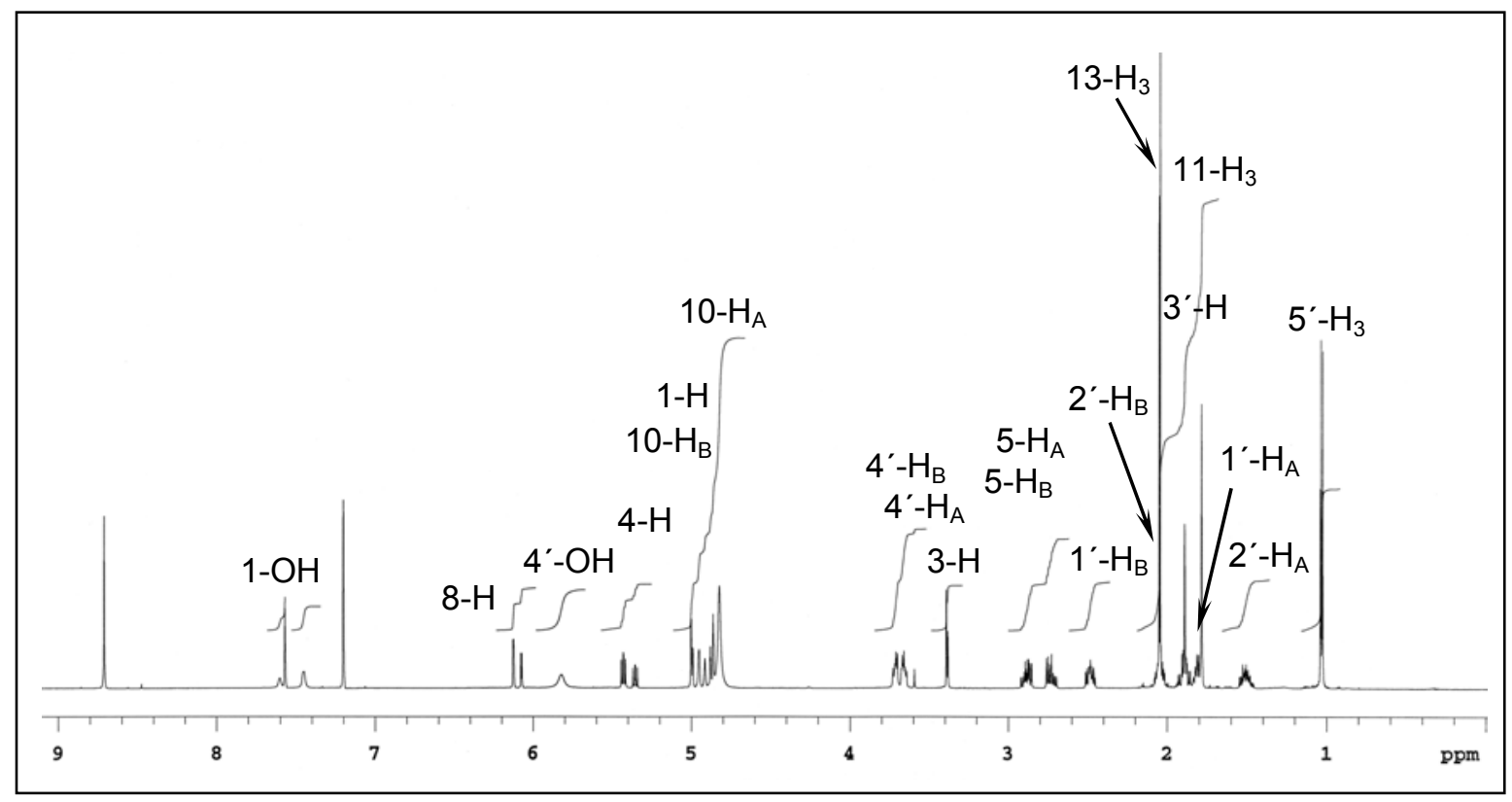

Abbildung 41: ${ }^{1} \mathrm{H}-\mathrm{NMR}-S p e k t r u m$ von $\underline{90}\left(600 \mathrm{MHz}, \mathrm{D}_{5}\right.$-Pyridin, $\left.35^{\circ} \mathrm{C}\right)$. 
Durch HPLC-MS-Analyse wurde deutlich, dass die Probe aus zwei Verbindungen bestand, die sich in ihrer Masse aber nicht unterschieden. Da alle Versuche einer Auftrennung der Isomere scheiterten, wurden die Strukturen von $\underline{90 \mathrm{a}}$ und $\underline{90 \mathrm{~b}}$ aus den Mischspektren aufgeklärt. Im Protonenspektrum wird anhand der Integrale deutlich, dass zwei Verbindungen im Verhältnis 3:2 vorliegen. Über ein HSQCExperiment konnten die einzelnen Signale der jeweiligen Verbindung zugeordnet werden. Die Ergebnisse sind in Tabelle 13 aufgelistet. Durch NMR-DatenAbgleich und zugeordnete HMBC-Korrelationen kann für $\underline{\mathbf{9 0}}$ das gleiche Kohlenstoffgerüst wie für Lupallen C (푸) angenommen werden. Der höhere $\mathrm{R}_{\mathrm{f}}$-Wert von 0.5 sagt vorher, dass die Anzahl der polaren Gruppen im Molekül aber verringert sein muss. Den Beweis liefern die chemischen Verschiebungen der Kohlen-

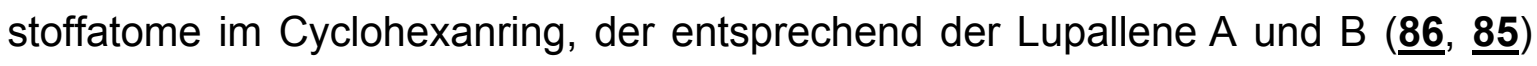
eine Epoxid-Funktion beinhaltet. Die Signale der zwei austauschbaren Protonen bei 7.45/7.61 und 5.83/5.83 stehen im Einklang mit diesem Befund. Unterstrichen wird der Strukturvorschlag durch das positive lon mit $345 \mathrm{~g} / \mathrm{mol}[322 \mathrm{~g} / \mathrm{mol}+\mathrm{Na}]^{+}$ im ESI-Massenspektrum.
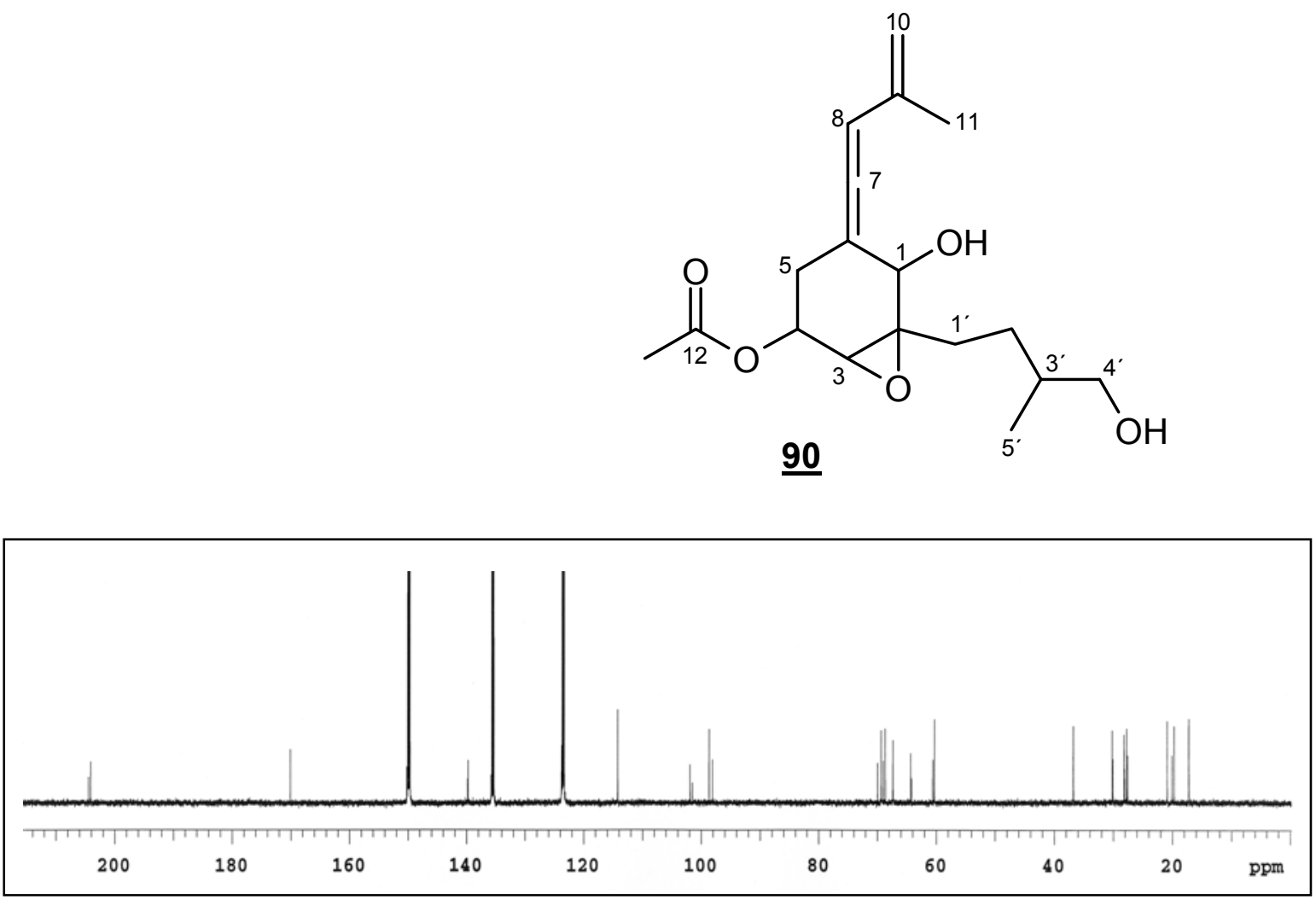

Abbildung 42: ${ }^{13} \mathrm{C}-\mathrm{NMR}-S p e k t r u m$ von $\underline{90}\left(125 \mathrm{MHz}, \mathrm{D}_{5}\right.$-Pyridin, $\left.35^{\circ} \mathrm{C}\right)$. 


\begin{tabular}{|c|c|c|c|c|}
\hline \multirow{2}{*}{$\mathrm{Nr}$. } & \multicolumn{2}{|c|}{$\underline{90 a}$} & \multicolumn{2}{|c|}{$\underline{90 \mathrm{~b}}$} \\
\hline & ${ }^{13} \mathrm{C}$ & ${ }^{1} \mathrm{H}$ & ${ }^{13} \mathrm{C}$ & ${ }^{1} \mathrm{H}$ \\
\hline $1-\mathrm{CH}$ & 69.33 & 4.95 & 69.95 & 4.91 \\
\hline $1-\mathrm{OH}$ & - & 7.45 & - & 7.61 \\
\hline C-2 & 64.30 & - & 64.17 & - \\
\hline $3-\mathrm{CH}$ & 60.56 & 3.39 & 60.28 & 3.38 \\
\hline $4-\mathrm{CH}$ & 68.62 & 5.43 & 68.99 & 5.36 \\
\hline $5-\mathrm{CH}_{2}$ & 28.18 & 2.74 & 28.08 & 2.71 \\
\hline & & 2.87 & & 2.90 \\
\hline C-6 & 101.90 & - & 101.49 & - \\
\hline C-7 & 204.10 & - & 204.40 & - \\
\hline $8-\mathrm{CH}$ & 98.65 & 6.13 & 98.08 & 6.08 \\
\hline C-9 & 139.72 & - & 139.82 & - \\
\hline $10-\mathrm{CH}_{2}$ & 114.20 & 4.86 & 114.20 & 4.88 \\
\hline & & 5.00 & & 4.99 \\
\hline $11-\mathrm{CH}_{3}$ & 19.70 & 1.78 & 20.06 & 1.89 \\
\hline C-12 & 170.09 & - & 170.09 & - \\
\hline $13-\mathrm{CH}_{3}$ & 20.87 & 2.04 & 20.87 & 2.05 \\
\hline $1^{\prime}-\mathrm{CH}_{2}$ & 30.19 & 1.90 & 30.08 & 1.90 \\
\hline & & 2.49 & & 2.48 \\
\hline $2^{\prime}-\mathrm{CH}_{2}$ & 27.74 & 1.52 & 27.58 & 1.50 \\
\hline & & 2.07 & & 2.07 \\
\hline $3^{\prime}-\mathrm{CH}$ & 36.75 & 1.81 & 36.80 & 1.81 \\
\hline $4^{\prime}-\mathrm{CH}_{2}$ & 67.32 & 3.65 & 67.36 & 3.66 \\
\hline & & 3.72 & & 3.72 \\
\hline $4^{\prime}-\mathrm{OH}$ & - & 5.83 & - & 5.83 \\
\hline $5^{\prime}-\mathrm{CH}_{3}$ & 17.23 & 1.03 & 17.20 & 1.04 \\
\hline
\end{tabular}

Tabelle 13: Vergleichende Betrachtung der 1D-NMR-Spektren von $\underline{90 \mathrm{a}}$ und $\underline{90 \mathrm{~b}}$.

Die Substanzen wurden in $\mathrm{D}_{5}$-Pyridin bei $35^{\circ} \mathrm{C}$ am $600 \overline{\mathrm{MHz}}$-Gerät gemessen.

Betrachtet man die NMR-Signale beider Isomere, liegen die größten Unterschiede im Bereich der Allengruppierung und an Position 1 des substituierten Cyclohexanringes. Ob nun eine axiale Isomerie bezüglich der Alleneinheit besteht oder das Stereozentrum an C-1 unterschiedlich konfiguriert ist, bleibt ungeklärt. Die Lösung des Problems wäre wiederum ein Kristall der Reinsubstanz, der, röntgenstrukturanalytisch vermessen, die abs. Stereochemie der Substanz wiedergeben würde. 


\section{Lupallen E (91)}

Der polarste der bisher isolierten Metabolite aus Fermenterkulturen fiel als weißer Feststoff bzw. farbloses Öl mit einer Ausbeute von bis zu $10 \mathrm{mg} / \mathrm{L}$ an. Die Verbindung ist optisch aktiv $\left([\alpha]_{\mathrm{D}}^{20}=+30^{\circ}(\mathrm{c}=0.10\right.$ in MeOH $\left.)\right)$ und weist im ESIMassenspektrum zwei Molpeaks im positiven $\left(321[\mathrm{M}+\mathrm{Na}]^{+}, 619[2 \mathrm{M}+\mathrm{Na}]^{+}\right)$und zwei im negativen Modus (297 [M-H], $\left.595[2 \mathrm{M}-\mathrm{H}]^{-}\right)$auf, was unter Berücksichtigung der NMR-Daten auf eine Summenformel von $\mathrm{C}_{16} \mathrm{H}_{26} \mathrm{O}_{5}$ schließen lässt. Im IR-Spektrum wird wieder eine scharfe Absorbtionsbande bei $1954 \mathrm{~cm}^{-1}$ gefunden, die zugehörigen ${ }^{13} \mathrm{C}-\mathrm{NMR}$ Signale der Allengruppierung finden sich bei $\delta_{\mathrm{C}}=97.9,103.8$ und $204.9 \mathrm{ppm}$. Dem ${ }^{13} \mathrm{C}-\mathrm{NMR}-$ Spektrum (in $\mathrm{CD}_{3} \mathrm{OD}$ ) sind außerdem 13 weitere Signale zu entnehmen, entsprechend dem schon bekannten Gerüst eines substituierten Cyclohexanringes. Im ${ }^{1} \mathrm{H}-\mathrm{NMR}-S p e k t r u m$ (in $\mathrm{CD}_{3} \mathrm{OD}$ ) zeigen sich die Signale der Alkylseitenkette bei $\delta_{H}=1.82 / 1.92,1.28 / 1.58,1.58$, 0.95 und 3.36/3.47. Die zwei Multiplett-Signale der endständigen Doppelbindung sind bei $\delta_{\mathrm{H}}=4.81$ und $4.90 \mathrm{zu}$ finden und weisen eine kleine Kopplung (ca. $0.5 \mathrm{~Hz})$ zur benachbarten Methylgruppe $\left(\delta_{\mathrm{H}}=1.78\right)$ auf. Das Dublett bei $\delta_{\mathrm{H}}=5.94$ wurde dem Allen-Proton zugewiesen. Dieses zeigt allylische Kopplungen $\left(J_{\mathrm{AX}}=4.0 \mathrm{~Hz}\right)$ mit dem ABMX-System der diastereotopen Methylengruppe an C-5, zentriert bei $\delta_{\mathrm{H}}=2.24 / 2.66$. Im $\mathrm{H}, \mathrm{H}$-COSY-Experiment werden weitere Kopplungen des als ddd erscheinenden Signals bei $\delta_{H}=4.03$ ppm offenbart, zum einen mit dem ABMX-System $\left(J_{A M}=12.5 \mathrm{~Hz}, J_{B M}=5.0 \mathrm{~Hz}\right)$, zum anderen mit dem

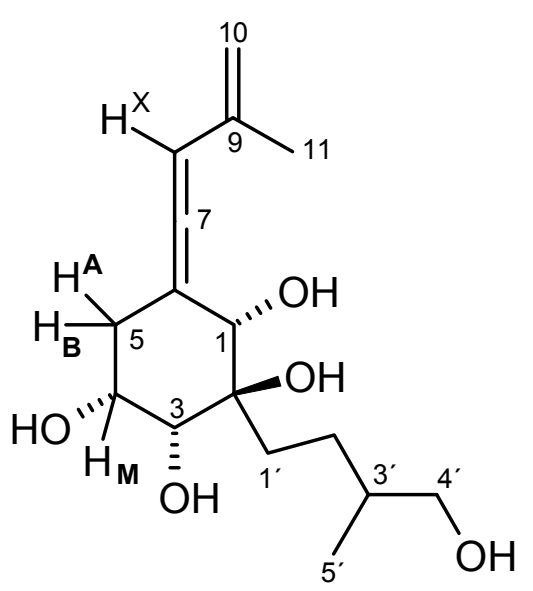

$\underline{91}$
Proton an $\mathrm{C}-3(\mathrm{~J}=3.0 \mathrm{~Hz})$, welches wiederum mit 1-H COSY-korreliert. Die Größe der Kopplungskonstanten geben für die Wasserstoffatome $\mathrm{A}$ und $\mathrm{M}$ eine axiale und für $3-\mathrm{H}$ eine äquatoriale Stellung im Sechsring vor. Die relative Stereochemie ergibt sich wie die in 87. Durch ein in $D_{5}$-Pyridin aufgenommenes Protonenspektrum werden auch die fünf austauschbaren Wasserstoffatome der freien Hydroxy-gruppen sichtbar. 


\section{Lupallen $F_{1}(\underline{92})$ und $F_{2}(\underline{93})$}

Die farblosen Metabolite öliger Konsistenz traten im Fermenter auf, wurden nach den ersten Reinigungsschritten als 1:1 Gemisch erhalten und durch präparative HPLC voneinander getrennt. Die massenspektrometrische Untersuchung der Reinsubstanzen führte zum gleichen Molgewicht von $280 \mathrm{~g} / \mathrm{mol}$, ersichtlich an den $[\mathrm{M}+\mathrm{Na}]^{+}$- und $[2 \mathrm{M}+\mathrm{Na}]^{+}$-Peaks bei 303 und 583. Bei Betrachtung der ${ }^{13} \mathrm{C}-\mathrm{NMR}$ Spektren hätte man annehmen können, dass es sich bei $\underline{92}$ und $\underline{93}$ um die gleiche Substanz handelt. Auch die ${ }^{1} \mathrm{H}-\mathrm{NMR}-S p e k t r e n$ beider Verbindungen sehen sich sehr ähnlich. Offensichtlich ist nur die chemische Verschiebung zweier Signale, das des 1-Hydroxyl-Wasserstoffatoms $\left(\delta_{H}=5.95\right.$ in $\underline{\mathbf{9 2}}, 6.12$ in $\left.\underline{\mathbf{9 3}}\right)$ sowie das als Dublett vom Triplett auftretende Signal $\left(\delta_{\mathrm{H}}=1.75\right.$ in $\left.\underline{\mathbf{9 2}}, 1.88 \mathrm{in} \underline{\mathbf{9 3}}\right)$, welches $1^{\prime}-\mathrm{H}_{\mathrm{A}}$ entspricht. Spekulativ wurden Isomere angenommen, die sich im Stereozentrum von C-1 und/oder C-2 unterscheiden. Für die weitere Strukturaufklärung wurden die NMR-Experimente von $\underline{92}$ benutzt.

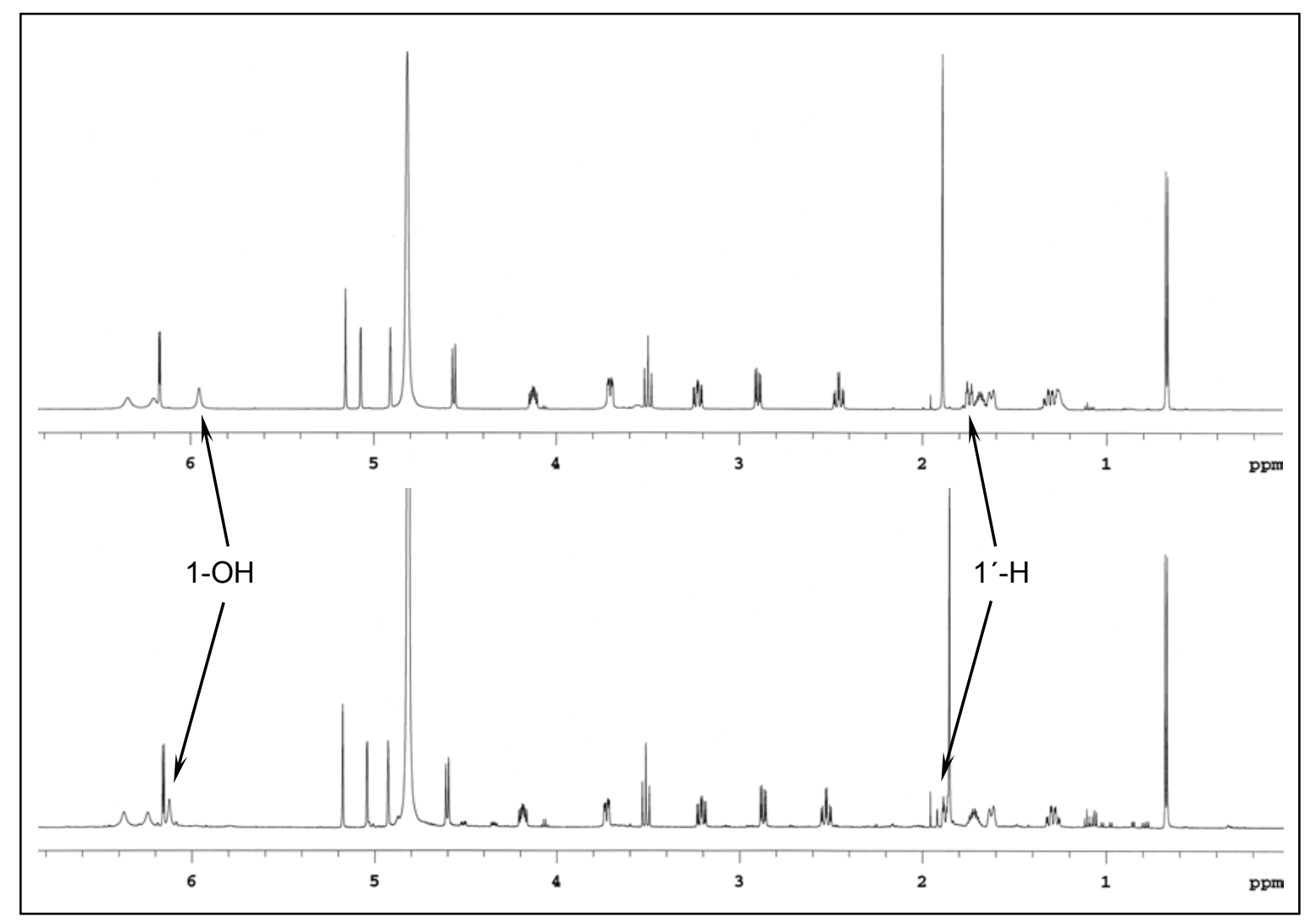

Abbildung 43: ${ }^{1} \mathrm{H}-\mathrm{NMR}-S p e k t r e n$ von $\underline{92}$ (oben) und $\underline{93}$ (unten), gemessen in $D_{5}$-Pyridin bei $35^{\circ} \mathrm{C}$ am $600 \mathrm{MHz}$-Gerät. 
In den 1D-NMR-Spektren konnte noch eine weitere Beobachtung gemacht werden. Es handelt sich um das gleiche Cyclohexangerüst analog zu Lupallen A (86), mit der vinylischen Allengruppierung an C-6 und der Alkylseitenkette an C-2. Die Signale der Alkylkette im ${ }^{1} \mathrm{H}-N M R-S p e k t r u m$ sind im Vergleich zu $\underline{\mathbf{8 6}} \mathrm{zu}$ höherem Feld verschoben, besonders deutlich ist dieses Phänomen an den Resonanzen der $5^{\prime}$-Methylgruppe $\left(\delta_{\mathrm{H}}=0.67\right)$, der $3^{\prime}$-Methingruppe $\left(\delta_{\mathrm{H}}=1.69\right)$ und der 2'-Methylengruppe $\left(\delta_{\mathrm{H}}=1.31 / 1.63\right)$ zu sehen. Ein HMBC-Experiment brachte die gleichen Korrelationen wie in $\underline{\mathbf{8 6}}$ zum Vorschein. Eine zusätzlich auftretende Kopplung zwischen dem quartären C-2 und den Protonen der 4'-Methylengruppe deutet einen Ringschluß zwischen C-2 und dem Sauerstoff an C-4' an. Eine Spektrensimulationen mit $\underline{\text { ACD Labs }}^{139}$ stand im Einklang mit diesem Strukturvorschlag.

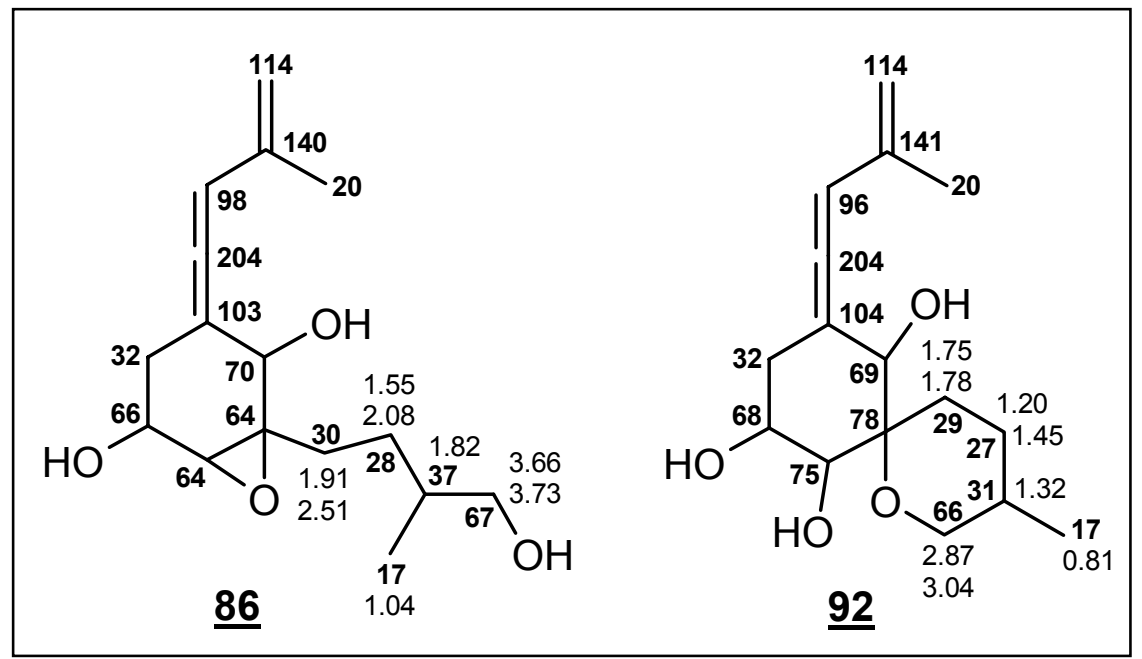

Abbildung 44: ${ }^{1} \mathrm{H}$ - und ${ }^{13} \mathrm{C}$-Verschiebungen in $\underline{\mathbf{8 6}}$ (real) und $\underline{92}$ (simuliert).

Anhand der Kopplungskonstanten aus den Protonenspektren konnte die relative Stereochemie im Cyclohexanring teilweise und im Seitenketten-Sechsring sogar vollständig aufgeklärt werden. Mit Hilfe eines NOESY-Experimentes, verdeutlicht in Abbildung 45, ließen sich sowohl die Anordnung der Ringe zueinander wie auch die relative Stereochemie an C-1 bestimmen. Das axiale Wasserstoffatom an C-4 $\left(\delta_{\mathrm{H}}=4.13\right)$ befindet sich in räumlicher Nähe zu der Methylengruppe an C-1' $\left(\delta_{H}=1.75 / 2.46\right)$, woraus eine axiale Anordnung für die Kohlenstoffkette und die äquatoriale Position für das Sauerstoffatom an C-2 geschlußfolgert werden kann. 
Fehlende NOESY-Korrelationen des axial angeordneten $3-\mathrm{H}\left(\delta_{\mathrm{H}}=4.56\right)$ in den Seitenkettenring stehen im Einklang mit diesem Befund. Für das Proton an C-1 $\left(\delta_{H}=5.15\right)$ lässt sich eine räumliche Lage nahe dem äquatorialen 1'-Methylenproton $\left(\delta_{\mathrm{H}}=1.75\right)$ und den beiden axialen Methylenprotonen an $\mathrm{C}^{\prime} 2^{\prime}\left(\delta_{\mathrm{H}}=1.31\right)$ und C-4' $\left(\delta_{H}=3.50\right)$ beschreiben. Deshalb kann es sich bei diesem Wasserstoffatom nur um einen äquatorialen Substituenten handeln. Zur axialen Chiralität können keine Aussagen getroffen werden.

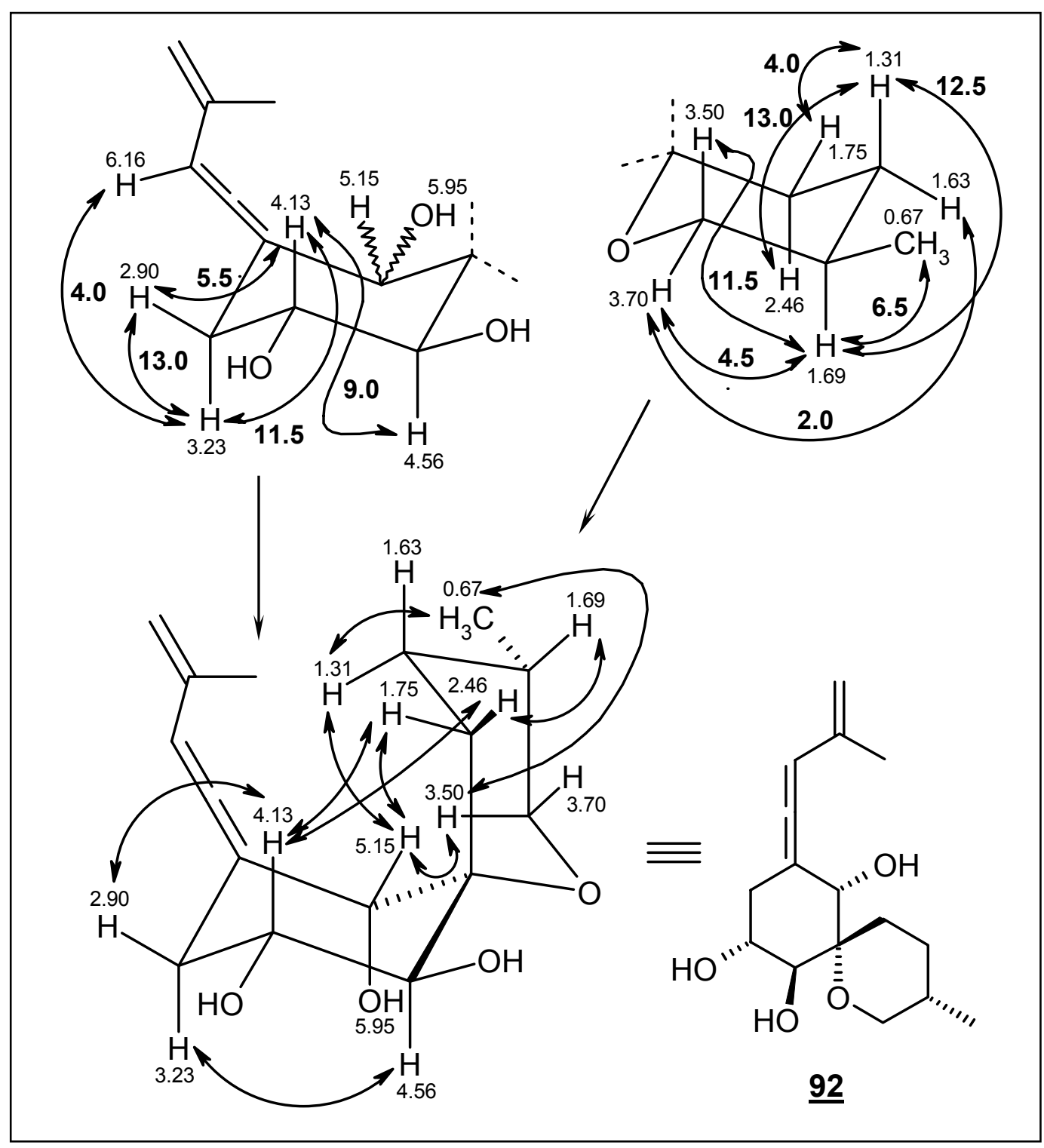

Abbildung 45: Mit Hilfe der ${ }^{1} \mathrm{H}$-Kopplungskonstanten wurde die Konformation der Sechsringe geklärt (oben). Die vollständige Bestimmung der relativen Stereochemie gelang über NOESY-Korrelationen (unten). 


\section{Lupaketon A $(\underline{94})$}

Aus der Fermentation im 10 L-Airlift-Fermenter ohne Überdruck konnte ein neuer Metabolit ohne Eigenfarbe mit einer Ausbeute von $5 \mathrm{mg} / \mathrm{L}$ isoliert werden. Später wurde er auch im Kulturfiltrat-Extrakt der Biostat-Fermentationen gefunden. Die Verbindung zeigt im Laufmittelsystem Chloroform/Methanol (9:1) einen $R_{f}-$ Wert von 0.2 und ist gut in Aceton und Methanol löslich. Im ESI-Massenspektrum finden sich die Molpeaks bei $321[\mathrm{M}+\mathrm{Na}]^{+}$und $619[2 \mathrm{M}+\mathrm{Na}]^{+}$, eine Hochauflösung des ersten Peaks ergibt die Summenformel $\mathrm{C}_{16} \mathrm{H}_{26} \mathrm{O}_{5}$.

Im IR-Spektrum lassen sich die Absorptionsbanden der $\mathrm{OH}-S t r e c k s c h w i n g u n g$ von Hydroxygruppen bei $3400 \mathrm{~cm}^{-1}$ und eine charakteristische Carbonyl-Bande bei $1679 \mathrm{~cm}^{-1}$ erkennen, die auf ein $\alpha, \beta$-ungesättigtes Keton hindeuten. Die fehlende Bande bei $1955 \mathrm{~cm}^{-1}$ schließt das Vorliegen eines Allens aus. Im ${ }^{13} \mathrm{C}-\mathrm{NMR}$ Spektrum sind wiederum 16 Kohlenstoffatome zu sehen. Im Hochfeldbereich und auch im Bereich zwischen 60 und 70 ppm ist eine deutliche Ähnlichkeit zu den Lupallenen bezüglich der Seitenkette und des Cyclohexanringes zu erkennen, nur mehr die Signale zweier Methylgruppen bei $\delta_{C}=27.2$ und 20.7 sowie das der Methingruppe bei $\delta_{C}=50.9$ sind nicht bekannt. Weiterhin sind ein $\mathrm{sp}^{2}-$ und zwei sp-Kohlenstoffatome $\left(\delta_{C}=124.6,154.8\right.$ und 202.7) auszumachen. Das bei tiefem Feld liegende Signal zeigt ein Keton an, während die anderen zu einer dreifachsubstituierten Doppelbindung gehören. Aufgrund der Beobachtungen im ${ }^{13} \mathrm{C}-\mathrm{NMR}$ Spektrum können trotz der fehlenden Alleneinheit gleiche Strukturteile angenommen und schon beschriebene Verbindungen zur Strukturaufklärung herangezogen werden. Aus dem ${ }^{1} \mathrm{H}-\mathrm{NMR}$-Spektrum kann auf den vollständigen Erhalt der Alkylseitenkette geschlossen werden. Das olefinische Proton bei $\delta_{H}=6.13$ spaltet mit $J=1.0 \mathrm{~Hz}$ zum Septett auf und liefert so die beiden Methylgruppen bei $\delta_{\mathrm{H}}=1.64(\mathrm{brs})$ und $2.14(\mathrm{~d}, J=1.0 \mathrm{~Hz})$ als weitere Substituenten der Doppelbindung. Es scheint, dass das Allen-Kohlenstoffatom im Cyclohexanring durch eine Methingruppe ersetzt wurde. Das zugehörige Proton ist durch das Signal eines Dublett vom Dublett vom Dublett bei $\delta_{H}=1.64$ gekennzeichnet. Als Kopplungspartner können eine Sauerstoff-substituierte Methingruppe $\left(\delta_{\mathrm{H}}=4.90\right.$, $J=9.0 \mathrm{~Hz})$ und eine diastereotope Methylengruppe $\left(\delta_{\mathrm{H}}=2.0-2.2, J=11.0,4.5 \mathrm{~Hz}\right)$ ausgemacht werden. Das Spinsystem wird durch zwei weitere Signale $\left(\delta_{\mathrm{H}}=4.67\right.$ 
und 3.50) vervollständigt. In nachfolgender Abbildung sind die COSY-Korrelationen noch einmal dargestellt. Über ein HMBC-Experiment gelingt die vollständige Verknüpfung der Fragmente.

In den Datenbanken $\underline{\text { Antibase }}^{47}$ und $\underline{\text { SciFinder }}^{76}$ gab es für diese Struktur keinen Eintrag, demnach ist sie als neu einzustufen.

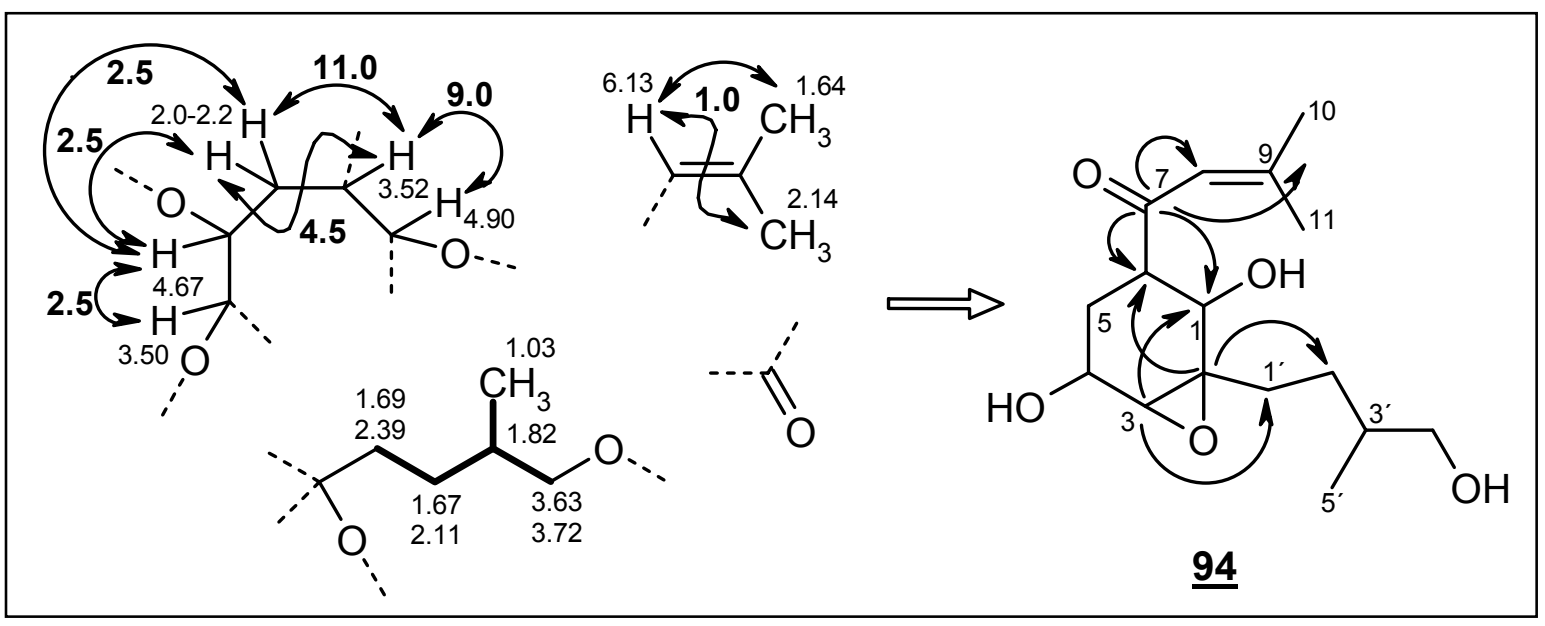

Abbildung 46: Aus Kopplungskonstanten und COSY-Korrelationen erhaltene Strukturfragmente, die über HMBC-Korrelationen zu $\underline{94}$ zusammengesetzt wurden.

\section{Lupaketon B $(\underline{95})$}

$\underline{95}$ fiel als farblose ölige Substanz an, die mit Anis pink-bräunlich und mit Orcin braun anfärbt. Dem ${ }^{1} \mathrm{H}-N M R-S p e k t r u m$ sind die Signale von drei Methylgruppen $\left(\delta_{H}=1.36,1.33,1.09\right)$ und fünf Methylengruppen zu entnehmen, von letzteren treten zwei $\left(\delta_{H}=3.69 / 3.73\right.$ und 2.45/2.57) als AB-System auf. Des Weiteren erscheinen die Resonanzen dreier Methingruppen, zwei davon an Sauerstoff gebunden, ebenso die zweier austauschbarer Protonen. Mit Hilfe der Kopplungskonstanten und eines $\mathrm{H}, \mathrm{H}-\mathrm{COSY}$-Experimentes konnten drei Spinsysteme ausgemacht werden, unter anderem eine Alkylkette, wie sie auch in den Lupallenen zu finden ist. Aus dem ${ }^{13} \mathrm{C}-\mathrm{NMR}$-Spektrum gehen außerdem fünf quartäre Kohlenstoffatome hervor, darunter eine heteroatomgebundene Isopropylgruppe $\left(\delta_{C}=81.0\right)$ sowie das C-Atom bei $\delta_{C}=57.9$, das die Bindungsstelle der Alkylkette mit dem Restmolekül darstellt. Bei tieferem Feld sind eine Sauerstoff-substituierte 
Doppelbindung $\left(\delta_{C}=107.3,164.8\right)$ und ein Keton $\left(\delta_{C}=191.3\right)$ zu erkennen. Nach einem HMBC-Experiment kann auf nachfolgende Struktur geschlossen werden.

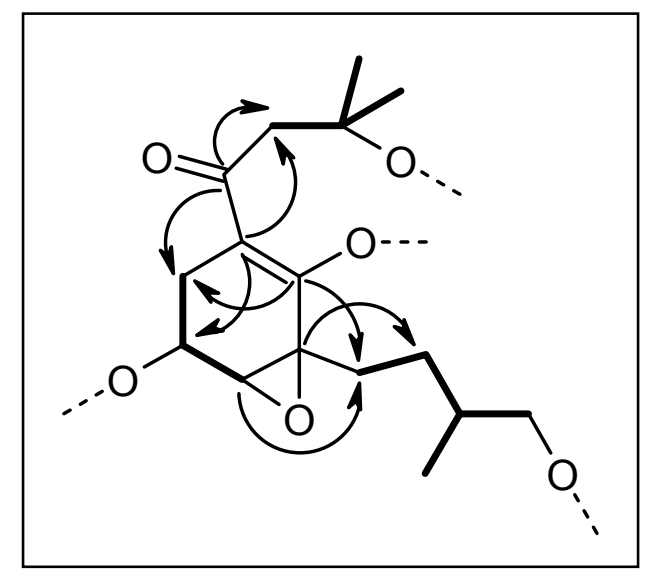

\begin{abstract}
Abbildung 47: Die drei Spinsysteme (dick) können durch HMBC-Korrelationen (Pfeile) mit den anderen Strukturfragmenten zum abgebildeten Molekül zusammengefügt werden.
\end{abstract}

Das ESI-Massenspektrum des Ketons zeigte aber nicht die erwartete Masse von $314 \mathrm{~g} / \mathrm{mol}$, sondern ein lon bei $319[\mathrm{M}+\mathrm{Na}]^{+}$, das zu einem Gewicht von $296 \mathrm{~g} / \mathrm{mol}$ führte. Die Aufnahme eines El-Massenspektrums bestätigte diesen Sachverhalt. Demnach befinden sich nur zwei freie Hydroxygruppen im Molekül, C-1 und C-9 sind über ein Sauerstoffatom zu einem Sechsring verbrückt.

Auch dieses Molekül kann wegen fehlender Einträge in Datenbanken (Antibase ${ }^{47}$ und $\underline{\text { SciFinder }}^{76}$ ) als neuer Naturstoff angesehen werden. Es gibt allerdings zwei Derivate mit der gleichen Tetrahydrochromanon-Grundstruktur, zum einen mit einer Isoprenyl-

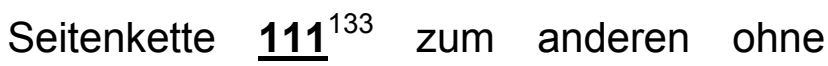
Seitenkette $\underline{110}^{132}$ (Siehe Seite 104). Die Bi-<smiles>CC(CO)CCC12OC1C(O)CC1C(=O)CC(C)(C)O[C@H]12</smiles>

$\underline{95}$ cyclen wurde jeweils zusammen mit einem

Allen $\underline{88}$ und $\underline{89}$ (Kapitel 6.3) isoliert. Für die Tetrahydrochromanone und die Allene wird der gleiche biogenetische Ursprung angenommen, näheres dazu in Kapitel 6.7. 


\section{3-Dimethylallyl-4-hydroxybenzoesäure $(\underline{96})$}

Der bei $254 \mathrm{~nm}$ UV-löschende Metabolit ist mit einem $\mathrm{R}_{\mathrm{f}}$-Wert von 0.25 (Chloroform/Methanol 9:1) den mittelpolaren Substanzen zuzuordnen. Er färbt mit Anis schlecht an und besitzt ein sehr übersichtliches ${ }^{1} \mathrm{H}-\mathrm{NMR}-\mathrm{Spektrum}$. Neben dem zum Dublett aufgespaltenem Signal einer enantiotopen $\mathrm{CH}_{2}$-Gruppe $\left(\delta_{\mathrm{H}}=3.70\right.$, $J=7.5 \mathrm{~Hz})$ und dem breiten Triplett-Signal eines olefinischen Protons $\left(\delta_{\mathrm{H}}=5.64\right.$, $J=7.5 \mathrm{~Hz}$ ) sind im Hochfeldbereich zwei Signale mit einer Intensität von drei zu sehen. Weiterhin findet man die Signale dreier aromatischer Wasserstoffatome, die angesichts des Aufspaltungsmusters einem 1,2,4-trisubstituierten Aromaten zuzuordnen sind. Dem ${ }^{13} \mathrm{C}-\mathrm{NMR}-S p e k t r u m$ sind außerdem vier quartäre Kohlenstoffatome zu entnehmen. Die chemische Verschiebung von 160.7 ppm lässt ein Phenol erwarten, 169.2 ist charakteristisch für eine aromatische Carbonsäure. Mit den ermittelten Strukturmerkmalen wurde eine Datenbankrecherche in $\underline{\text { Antibase }}^{47}$ durchgeführt, die 68 Einträge erbrachte. Beste Übereinstimmung zeigt das gefundene Molekül mit den experimentellen Daten von 4-Hydroxy-3-(3'-methyl-2'butenyl)-benzoesäure, obgleich ein quartäres Kohlenstoffatom im ${ }^{13} \mathrm{C}-N M R$-Spektrum von $\underline{96}$ vermisst wurde. Ein El-Massenspektrum bestätigte die Molmasse von $206 \mathrm{~g} / \mathrm{mol}$. Die Hochauflösung des Molekülions lieferte die Summenformel $\mathrm{C}_{12} \mathrm{H}_{14} \mathrm{O}_{3}$. Das charakteristische Fragment bei $\mathrm{m} / \mathrm{z}=151$ [100\%, M-55] entsteht durch Abspaltung von $\mathrm{CH}=\mathrm{C}\left(\mathrm{CH}_{3}\right)_{2}$ aus der Seitenkette.

Der Naturstoff wurde schon 1991 als Pilzmetabolit aus Discula sp. beschrieben. ${ }^{140}$ Dieser Pilz gelangte Bekanntheitsgrad durch Anthraknose bei blühenden Hornsträuchern (Hartriegel, lat.: Cornus). Discula sp. verursacht dabei Blattnekrosen sowie Baumgeschwüre und führt zum Absterben des Gewächses, wenn der Stamm komplett vom Geschwür umgeben ist. Von den phytotoxischen Metaboliten des Stammes war $\underline{96}$ das in höhster Konzentration enthaltene Toxin.<smiles>CC(C)=CCc1cc(C(=O)O)ccc1O</smiles> 


\section{4-Hydroxy-3-(3'-hydroxy-3'-methyl-but-1-enyl)-benzoesäure (무)}

Die Verbindung fiel bei der Analyse der Kulturfiltrat-Extrakte aus den BiostatFermentern als neue, mit Anis und Ehrlich bläulich anfärbende Zone im Dünnschichtchromatogramm auf und konnte als farbloses Öl isoliert werden. Bei einer HPLC-MS-Untersuchung war der Substanzpeak bei einer Retentionszeit von 11.03 min auszumachen, die Molmasse ergab sich zu $222 \mathrm{~g} / \mathrm{mol}$, ersichtlich durch die lonenpeaks bei $223[\mathrm{M}+\mathrm{H}]^{+}$und $467[2 \mathrm{M}+\mathrm{Na}]^{+}$. Das ${ }^{1} \mathrm{H}-\mathrm{NMR}-$ Spektrum zeigt neben den Singuletts zweier Methylgruppen im Hochfeldbereich das Vorliegen einer cis-Doppelbindung an $\left(\delta_{\mathrm{H}}=6.42\right.$ und 5.64, $\left.\mathrm{d}, J=10.0 \mathrm{~Hz}\right)$. Die Resonanzen bei $\delta_{H}=8.24,8.14$ und 6.99 repräsentieren einen 1,2,4-trisubstituierten Aromaten. Im ${ }^{13} \mathrm{C}-N M R-S p e k t r u m$ tauchen zusätzlich fünf Signale quartärer Kohlenstoffatome auf, deren chemische Verschiebungen weiter Einblicke in die Struktur gewähren. Das Molekül beinhaltet demnach eine Carbonsäure $\left(\delta_{C}=168.1\right)$, einen Benzolring $\left(\delta_{C}=120.6,124.6\right)$ mit phenolischer OH-Gruppe $\left(\delta_{C}=164\right)$ sowie eine Sauerstoffsubstituierte Isopropylfunktionalität. In Anlehnung an das Substitutionsmuster von $\underline{96}$ ergibt sich die Konstitution von der beschriebenen Verbindung zu $\underline{97}$. Die Struktur ist in keiner der benutzten Datenbanken bekannt.

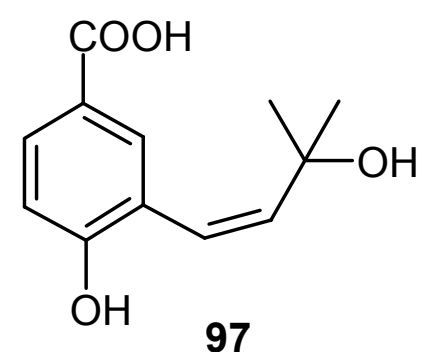

\subsection{Derivatisierung der Allene}

Für die meisten Allen-Derivate des Stammes WDMH46 konnte die relative Stereochemie aus NMR-Experimenten abgeleitet werden. Für die Klärung der absoluten Konfiguration wäre ein Kristall von Nöten, um eine Röntgenstrukturanalyse durchzuführen. Bislang war es nicht gelungen, auch nur von einem der Allene einen Kristall zu züchten. In Anbetracht dieser Tatsache sollte versucht werden, die Tendenz zur Kristallbildung durch Veresterung mit Brombenzoesäuren zu verbessern. 
Da es sich bei den allenischen Naturstoffen um mehrfach hydroxylierte Systeme handelt, wurde zunächst eine Acetylierungsreaktion durchgeführt, um Einblicke in die Reaktivität der einzelnen Hydroxygruppen zu bekommen. Als Allen-Edukt wurde sich für Lupallen C (푸) entschieden, da der Metabolit von vornherein einen $\mathrm{OH}-\mathrm{Substituenten} \mathrm{weniger} \mathrm{enthielt.}$

\subsubsection{Acetylierung von Lupallen $\mathrm{C}$}

Die Umsetzung von Lupallen C (구) mit Essigsäureanhydrid/Pyridin lieferte nach zwei Stunden ein Produkt (요), das nach Chromatographie an Sephadex LH-20 (Aceton) mit 54\% Ausbeute in Reinsubstanz erhalten werden konnte. Durch die Acetylierung wurde die Polarität der Verbindung erwartungsgemäß herabgesetzt. Das ${ }^{13} \mathrm{C}-N M R-S p e k t r u m$ ist durch das zusätzliche Auftreten einer Carbonylfunktion $\left(\delta_{C}=170.8\right)$ und einer Methylgruppe $\left(\delta_{C}=21.1\right)$ gekennzeichnet, demnach ist nur eine der freien $\mathrm{OH}-G r u p p e n$ eine Reaktion eingegangen. Das gleiche Ergebnis lieferte ein DCl-Massenspektrum, dessen lonen bei $\mathrm{m} / \mathrm{z}=400\left[\mathrm{M}+\mathrm{NH}_{4}\right]^{+}$und 783 $\left[2 \mathrm{M}+\mathrm{NH}_{4}\right]^{+}$ein Monoacetat bestätigten. Im ${ }^{1} \mathrm{H}-\mathrm{NMR}$-Spektrum ist die zusätzliche Methylgruppe bei $\delta_{H}=1.94 \mathrm{zu}$ sehen. Durch die Tieffeldverschiebung des ABMSignals bei $\delta_{H}=3.70 / 3.78 \mathrm{zu}$ 3.98/4.11 wurde die Acetoxygruppe an der 4'-Methylengruppe lokalisiert.<smiles>C=C(C)C=C=C1CC(OC(C)=O)C(O)C(O)(CCC(C)[CH]OC(C)=O)C1O</smiles>

$\underline{98}$ 


\subsubsection{Umsetzung von Lupallen $\mathrm{C}$ mit ortho-Brombenzoesäure}

$11.4 \mathrm{mg} \underline{87}$ wurden mit 2-Brombenzoesäure in Gegenwart von EDCl und DMAP zur Reaktion gebracht und die Umsetzung des Allens durch DC-Kontrolle verfolgt. Obwohl die dünnschichtchromatographische Analyse des Rohproduktes nur eine ca. 60 \%ige Umsetzung zeigte, wurde die Reaktion nach vier Stunden abgebrochen und der Ansatz aufgearbeitet. Mit Hilfe einer Kieselgel-Säule $\left(\mathrm{CH}_{2} \mathrm{Cl}_{2} /\right.$ $\mathrm{MeOH}$ 19:1) konnte neben dem Benzoylierungsprodukt auch Edukt zurückgewonnen werden. Eine abschließende Chromatographie an Sephadex $\mathrm{LH}-20\left(\mathrm{CH}_{2} \mathrm{Cl}_{2} /\right.$ $\mathrm{MeOH} 1: 1)$ lieferte von aufgetragenen $11.8 \mathrm{mg}$ verunreinigter Substanz $5.9 \mathrm{mg}$ reines Brombenzoat ( $\underline{99})$ mit einer Ausbeute von $34 \%$.

Entsprechend der Acetylierungsreaktion wurde ein Derivat erwartet, bei dem die Hydroxygruppe an C-4' mit 2-Brombenzoesäure verestert ist. Das ${ }^{13} \mathrm{C}-N M R-S p e k-$ trum des Produktes war genau um den Signalsatz eines aromatischen Systems mit Esterfunktion ergänzt. Der 1,2-disubstituierte Aromat spiegelte sich im ${ }^{1} \mathrm{H}$ NMR-Spektrum durch die Signale mit in Abbildung 48 angegebenem Kopplungsmuster wieder. Die Resonanzen der 4'-Methylengruppe treten wieder tieffeldverschoben auf und weisen die veresterte Hydroxygruppe an C-4'. Die Substitution wird im ESI-Massenspektrum durch zwei lonenpeaks bei $545[\mathrm{M}+\mathrm{Na}]^{+}$und 1068 $[2 \mathrm{M}+\mathrm{Na}]^{+}$bestätigt.

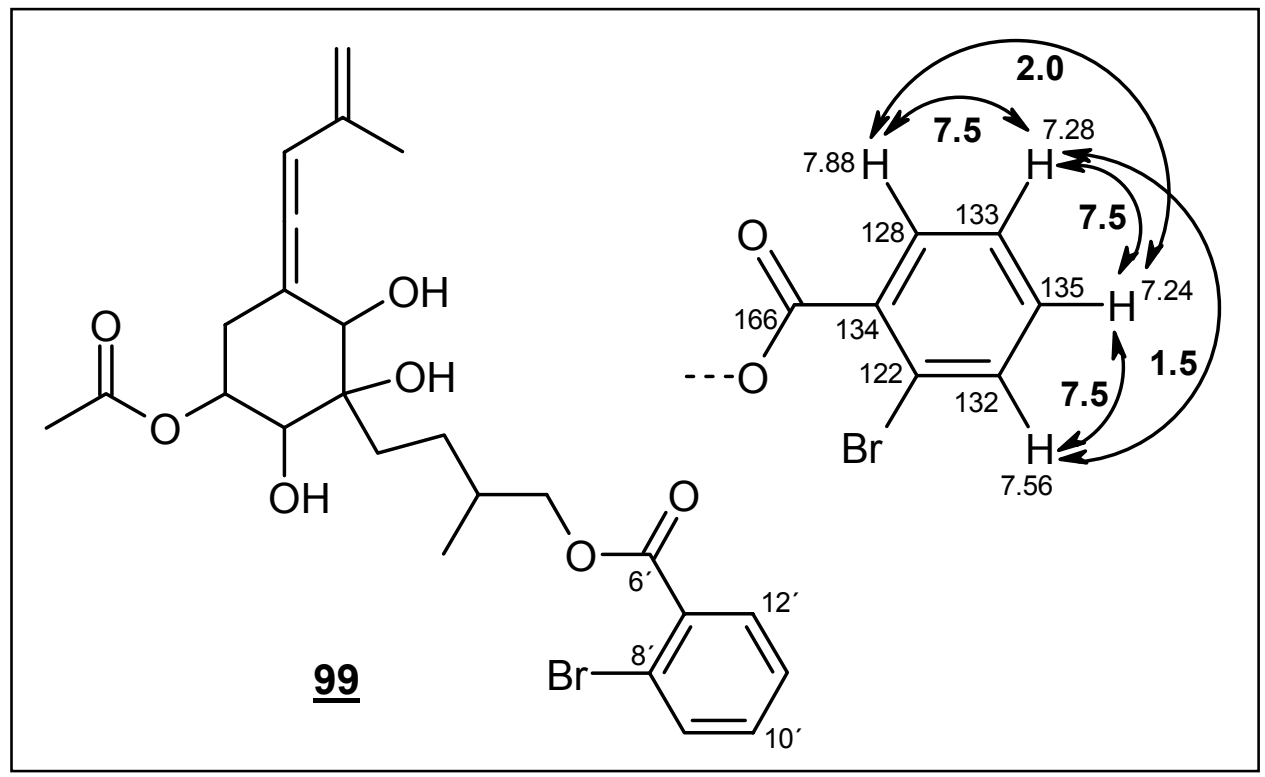

Abbildung 48: Das Produkt ( $\underline{99})$ aus der Umsetzung von 87 mit 2-Brombenzoesäure sowie das Kopplungsmuster der hinzugekommenen NMR-Signale. 


\subsubsection{Umsetzung von Lupallen A mit para-Brombenzoesäure}

Da das Brombenzoat (모) von Lupallen C in öliger Konsistenz erhalten wurde und auch nicht zu kristallisieren war, wurde mit 4-Brombenzoesäure als Reagenz ein neuer Versuch gestartet. T. BENDER, ein Mitarbeiter der Arbeitsgruppe GROND, hatte mit dem para-Brombenzoat einer seiner Substanzen gute KristallisationsErfahrungen gemacht. Als weitere Änderung wurde Lupallen A ( $\underline{\mathbf{8 6}}$ ) als Substrat ausgewählt. Die Umsetzung von $\underline{\mathbf{8 6}}$ erfolgte analog der Brombenzoylierung von Lupallen C (구) und war in der dünnschichtchromatographischen Kontrolle durch das Auftauchen von vier bis fünf Produktzonen und das augenscheinliche Verschwinden des Eduktes gekennzeichnet. Aus den eingesetzten $50.3 \mathrm{mg}$ an $\underline{\mathbf{8 6}}$ erhielt man nach der Aufarbeitung einen schwach gelblichen, viskosen Rückstand von $116.0 \mathrm{mg}$. Die anschließende Säulenchromatographie an Kieselgel lieferte sechs Fraktionen. In Fraktion 6 konnten 3.2 mg Edukt nachgewiesen werden, Fraktion 3 und 4 enthielten ein Minderprodukt (3.3 und $3.8 \mathrm{mg}$ ) in unterschiedlich stark verschmutztem Zustand, auf dessen Aufreinigung verzichtet wurde. Der Inhalt der 5. Fraktion $(22.0 \mathrm{mg})$ bestand aus zwei Produkten, die sich nicht weiter auftrennen ließen. Für die Fraktionen 1 und 2, die im Kolben als zusammenhängende weiße Nadeln kristallisierten, konnte über ein ${ }^{1} \mathrm{H}-\mathrm{NMR}$-Experiment auf das gleiche Brombenzoat geschlossen werden. Die sowohl in F1, noch stärker in F2 auftretende Verunreinigung erwies sich als 4-Brombenzoesäuremethylester. Beide Fraktionen wurden vereinigt und über Sephadex LH-20 (Dichlormethan) chromatographiert. Mit dieser Säule ist es gelungen, den Methylester nahezu vollständig vom gewünschten Produkt zu trennen.

Die lipophile Substanz $\underline{100}(13.6 \mathrm{mg})$ weist in Chloroform/Methanol 9:1 einen $\mathrm{R}_{\mathrm{f}}$-Wert von 0.67 auf und wurde als Kristallbrei erhalten. Im ${ }^{1} \mathrm{H}-\mathrm{NMR}$-Spektrum sind die Signale des para-substituierten Aromaten im Bereich von 7.56-7.67 und 7.98-8.02 ppm zu sehen, die Integrale zeigen aber mehr als vier Protonen an. Die Vermutung wird dadurch bestätigt, dass nicht nur das ABM-Signal der 4'-Methylengruppe analog zu $\underline{99}$ tieffeldverschoben gefunden wird, sondern auch eine Methingruppe bei tieferem Feld erscheint. Durch einen Spektrenvergleich mit dem Edukt (Signalverschiebung von $\delta_{H}=4.59$ nach 5.67) kann die Hydroxygruppe an C-4 als weiterer Reaktionspartner der Säure ausgemacht werden. Das ${ }^{13} \mathrm{C}-\mathrm{NMR}-$ Spektrum ist um die zusätzlich auftretenden Signale des para-Bromsäureesters 
erweitert, die aber nicht einheitlich, sondern als doppelter Signalsatz auftreten. Eine El-Massenanalyse zeigte den erwarteten Molpeak bei $\mathrm{m} / \mathrm{z}=646$. Bis zum jetzigen Zeitpunkt ist es nicht gelungen, von $\underline{100}$ einen vermessbaren Kristall zu erhalten.

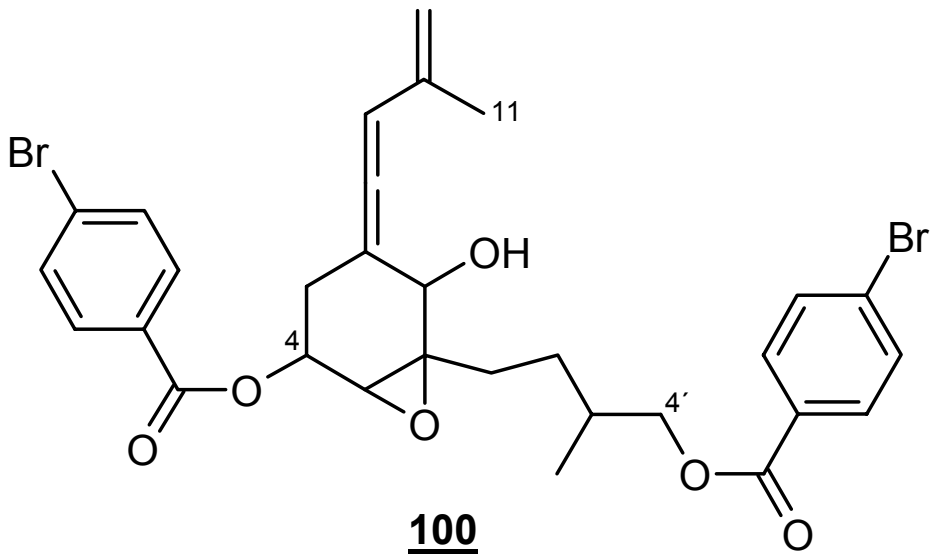

Der Stamm WDMH46 hat sich als äußerst vielfältig erwiesen. Die im chemischen Screening als pinkfarbene Spots (Anis) im DC aufgefallenen Substanzen konnten als Allene charakterisiert werden. Zusätzlich wurden vier weitere Metabolite vom Stamm gebildet, für die aufgrund ähnlicher Strukturteile der gleiche biogenetische Ursprung angenommen werden kann. (Siehe Abbildung 49) Durch Fütterungsexperimente galt es nun zu prüfen, ob die Verbindungen Vorläufer auf dem Weg zum Lupallen-Gerüst oder als Shunt-Produkte einzustufen sind.

\subsection{Biogeneseuntersuchungen}

\subsubsection{Bekanntes zur Biosynthese von Allenen}

Zur Biosynthese von Allenen ist nicht allzuviel bekannt. In den bisherigen Biosynthesearbeiten wurde im wesentlichen von zwei Ansätzen ausgegangen.

a) Allene entstehen durch eine Dien-Oxidation und sind BiosyntheseIntermediate auf dem Weg zu Acetylenen. ${ }^{111 \mathrm{c}}$

b) Acetylene bilden den Ursprung der Allene, diese sind aber nur ein Zwischenprodukt in der Chromanon-Biosynthese. ${ }^{132}$ 


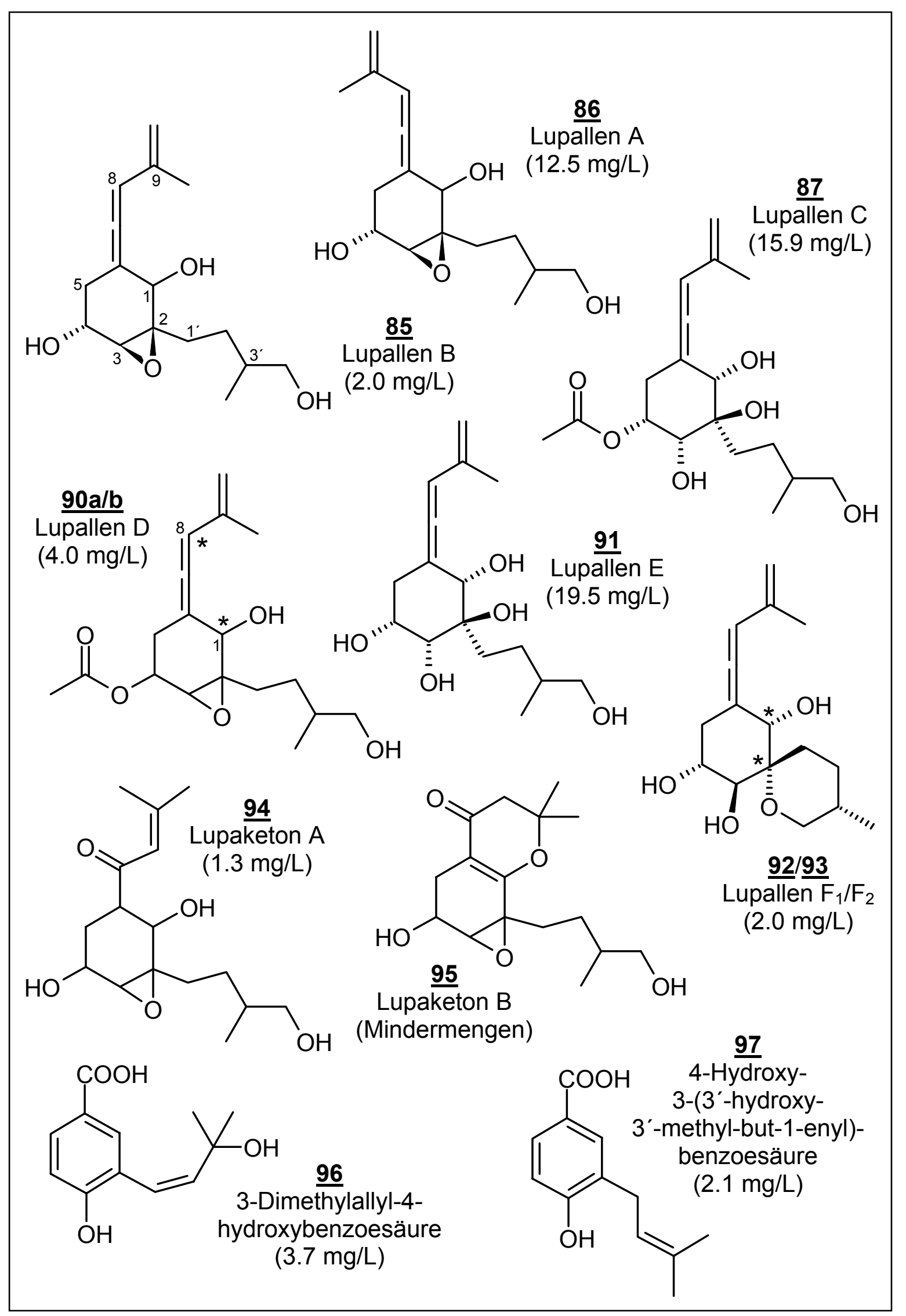

Abbildung 49: Aus dem Stamm WDMH46 konnten 12 Sekundärmetabolite isoliert und charakterisiert werden. 11 davon sind neue Naturstoffe. 
Das nachfolgende Kapitel soll Einblicke in diese Untersuchungen geben. Deshalb werden die verschiedenen Biosyntheseansätze kurz vorgestellt.

Schon sehr früh wurde spekuliert, dass es einen biogenetischen Zusammenhang zwischen acetylenischen und allenischen Carotinoiden gibt. ${ }^{141}$

Man fand heraus, dass es im Prinzip möglich ist, durch Ringepoxidierung in Nachbarschaft zu einer Acetylengruppe, gefolgt von reduktiven Prozessen, ein Allensystem zu generieren. ${ }^{141}$<smiles>[R]C#CC1=C(C)CC(O)CC1(C)C</smiles>

Gestützt wird diese Hypothese durch die entsprechenden Naturstoffe, als Beispiele seien hier das Diatoxanthin $(\underline{\mathbf{1 0 1}})^{142}$ und Fucoxanthin $(\underline{\mathbf{7 7}})^{118,119}$ aufgeführt.

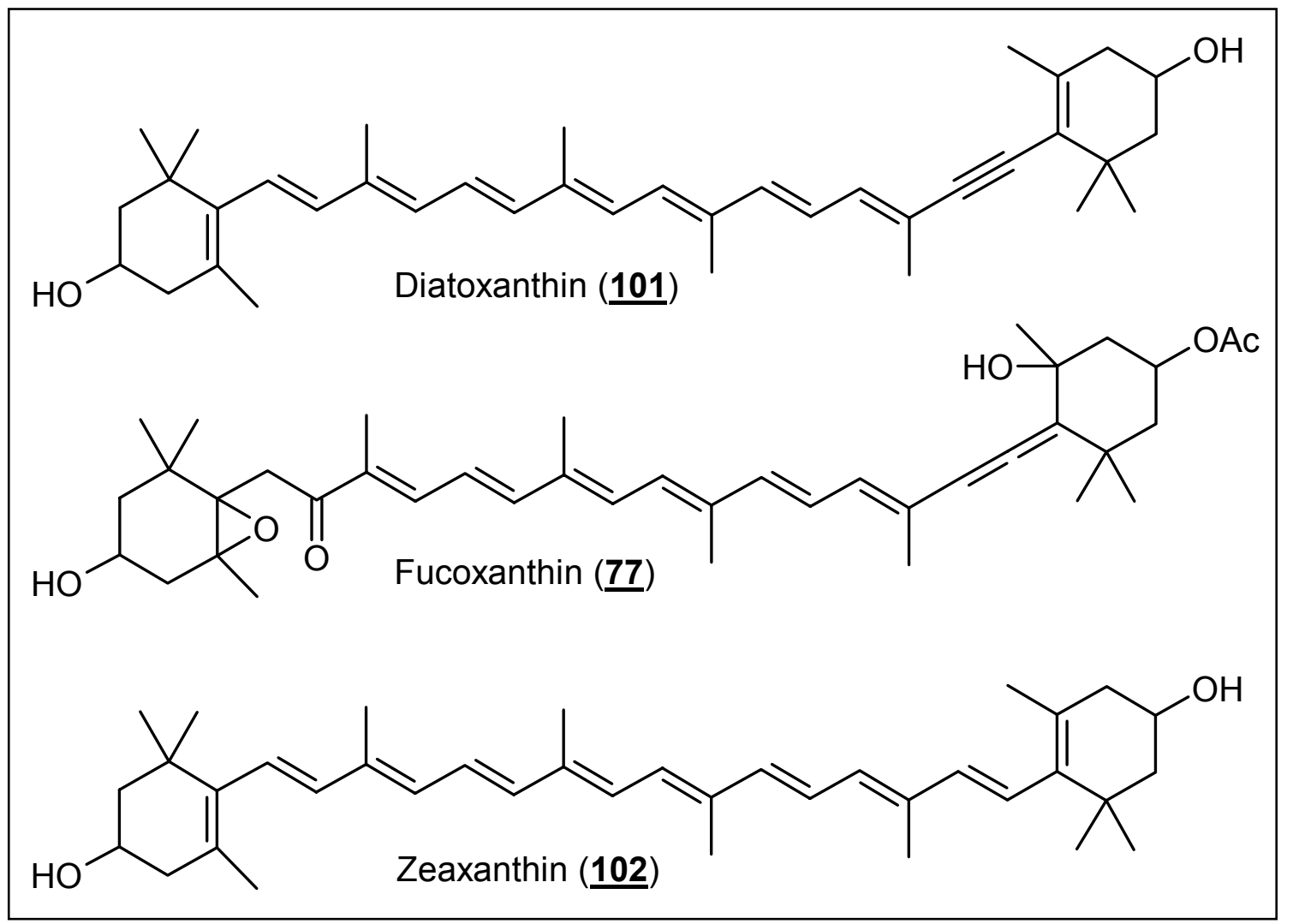

Abbildung 50: Natürliche Carotinoide, die möglicherweise über eine verknüpfte Biosynthese entstehen. 
Eine andere Idee war, Zeaxanthin (102) als biosynthetischen Vorläufer von $\underline{77}$ anzunehmen. Drei Arbeitsgruppen ${ }^{143-145}$ beschäftigten sich zeitgleich und unabhängig voneinander mit photosensibilisierten Oxigenierungen von $\beta$-lonol oder ähnlichen Systemen. Durch diese Reaktion entstehen allenische Hydroperoxide, die zum allenische Alkohol reduziert werden können.

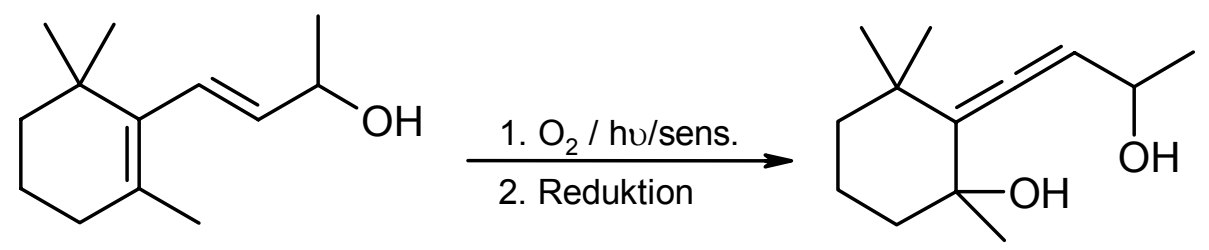

Diese Ergebnisse haben zu der Annahme geführt, dass es analoge Prozesse in der Natur geben könnte, die Zeaxanthin (102) in allenischen Carotinoide umzuwandeln vermögen. Der diskutierte Biosyntheseweg geriet ins Wanken, als durch photosensibilisierte Oxigenierung des $\beta$-lonol $\underline{103}\left(\mathrm{R}=\mathrm{CHCH}_{3} \mathrm{OH}\right)$ ein Allen (104) produziert wurde, dessen relative Konfiguration gegensätzlich zu der in Fucoxanthin (7ㅡ) gefundenen auftrat. ${ }^{146}$ Eine Erklärung war, dass das anfänglich bebildete System in der Natur eine Photoisomerisierung eingeht. ${ }^{147}$ Bestrahlung eines allenischen Ketons $\left(\mathrm{R}=\mathrm{COCH}_{3}\right)$ induzierte eine Gleichgewichtseinstellung beider Isomere und erbrachte eine 1:1-Mischung.<smiles>CC1=C(/C=C/F)C(C)(C)CC(O)C1</smiles>

$\underline{103}$

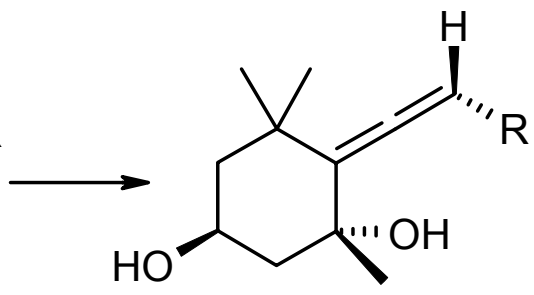

$\underline{104}$

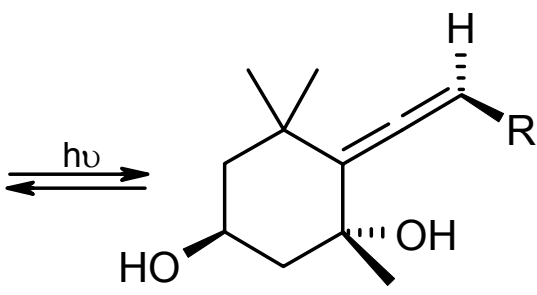

$\underline{105}$

Die Oxidation eines Dien-Precursors generiert demnach ein (S)-Allen, welches dann zum natürlichen $(R)$-Allen isomerisiert, wobei die Prozesse in der Natur nicht notwendigerweise photoinduziert ablaufen müssen. Für diese Biosynthesehypothese spricht, dass in einer Seetang-Probe geringe Mengen des (S)-Allenisomers von Fucoxanthin (프) gefunden wurden. ${ }^{148}$ 
1981 berichtete I.A. SWIFT und B.V. MILBORROW über die „Stereochemie der Allenbiosynthese und die Bildung des acetylenischen Carotinoids Diadinoxanthin aus Neoxanthin“. ${ }^{149}$ Sie konnten durch Fütterungsexperimente an intakten Zellen der Alge Amphidium carterae (Dinophyceae) und auch am daraus gewonnenen zellfreien System einen Einbau von $(2 R S, 3 R)-\left[2-{ }^{14} \mathrm{C}, 2-{ }^{3} \mathrm{H}_{2}\right]-$ Mevalonat in Lucopen

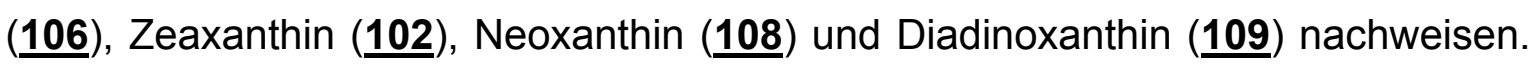
Die Markierung war an den Kohlenstoffatomen C-1, C-4, C-8 und C-12 und den daran gebundenen Wasserstoffatomen jeder Carotinoid-Hälfte zu verzeichnen. Die Anreicherungen sind exemplarisch für alle isolierten Substanzen für das Zeaxanthin $\left({ }^{14} \mathrm{C}=\mathbf{a}\right)$ und Neoxanthin $\left({ }^{3} \mathrm{H}=\bullet\right)$ in Abbildung 51 dargestellt. Dem Experiment ist weiter zu entnehmen, dass ein Wasserstoffatom von C-2 des Mevalonat in die Allengruppierung an C-8 eingebaut wurde. Diese Beobachtung schließt eine Neoxanthin-Allen-Bildung aus dem Diadinoxanthin-Acetylen aus, da sie mit dem Verlust der ${ }^{3} \mathrm{H}$-Markierung an $\mathrm{C}-8$ einhergehen würde. Denkbar ist auch der umgekehrte Fall, die Umwandlung des Allens in ein Acetylen-Derivat. Der einfache Verlust eines Tritiums an C-8 in Diadinoxanthin geht mit der Annahme konform. Aus Ergebnissen der Fütterung wurde von I.A. SWIFT und B.V. MILBORROW folgendes Biosyntheseschema abgeleitet. ${ }^{149}$ 


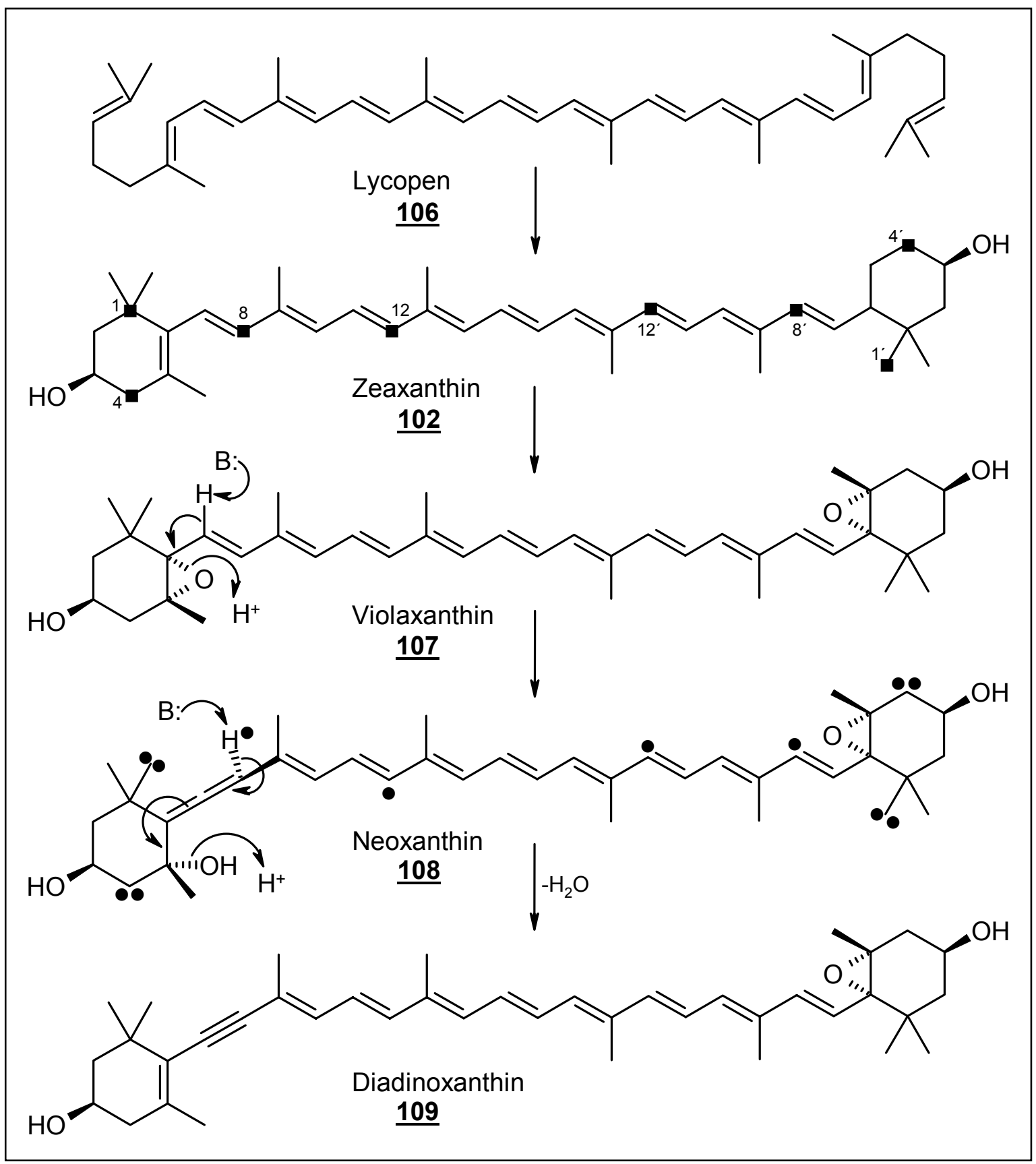

Abbildung 51: Postulierte Biosynthese carotinoider Allene und Acetylene $\left({ }^{14} \mathrm{C}=\mathbf{\square},{ }^{3} \mathrm{H}=\bullet\right) .{ }^{149}$

Wenn man sich die Publikationen zu den Vinylallenen $\underline{\mathbf{8 8}}^{132}$ und $\underline{\mathbf{8 9}}^{133}$ betrachtet, fällt auf, dass diese immer in Kombination mit den strukturverwandten Tetrahydrochromanon-Derivaten gefunden wurden. Genau die gleiche Erfahrung wurde auch beim Stamm WDMH46 gemacht. Es liegt demnach die Vermutung nahe, dass beide Metabolite dem gleichen Biosyntheseweg entspringen. 


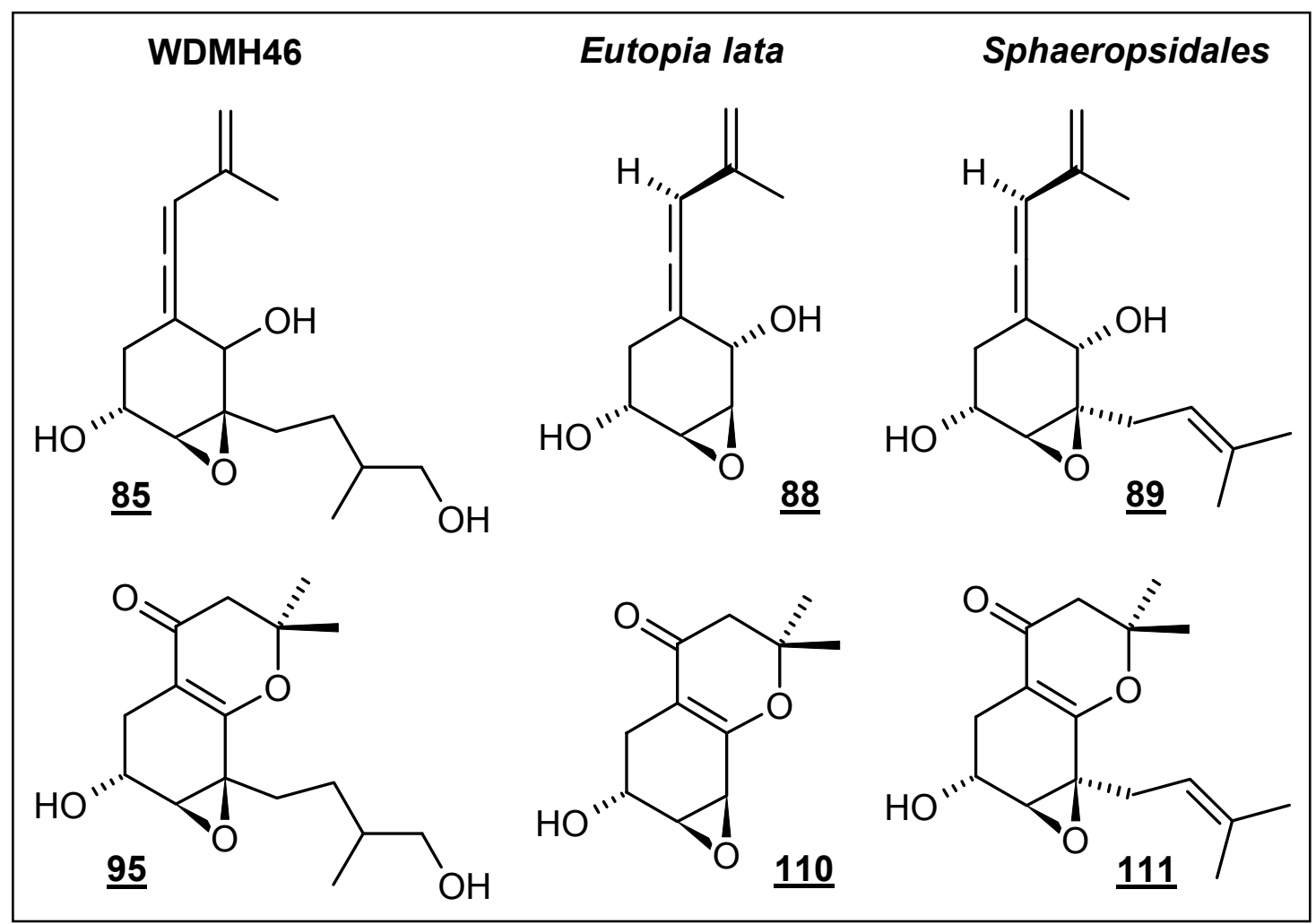

Abbildung 52: Sekundärmetabolite aus Eutopia lata, Sphaeropsidales und WDMH46.

R. TABACCHI und seine Mitarbeiter brachten die zwei Metabolite mit dem ebenfalls aus Eutopia lata isolierten aromatischen Acetylen ${ }^{150} \underline{112}$ in Zusammenhang und postulierten nachfolgendes Biosyntheseschema. Sie betrachteten das Allen $\underline{\mathbf{8}}$ als Schlüsselintermediat in der Transformation vom Acetylen zum Tetrahydrochromanon-Derivat. ${ }^{132}$

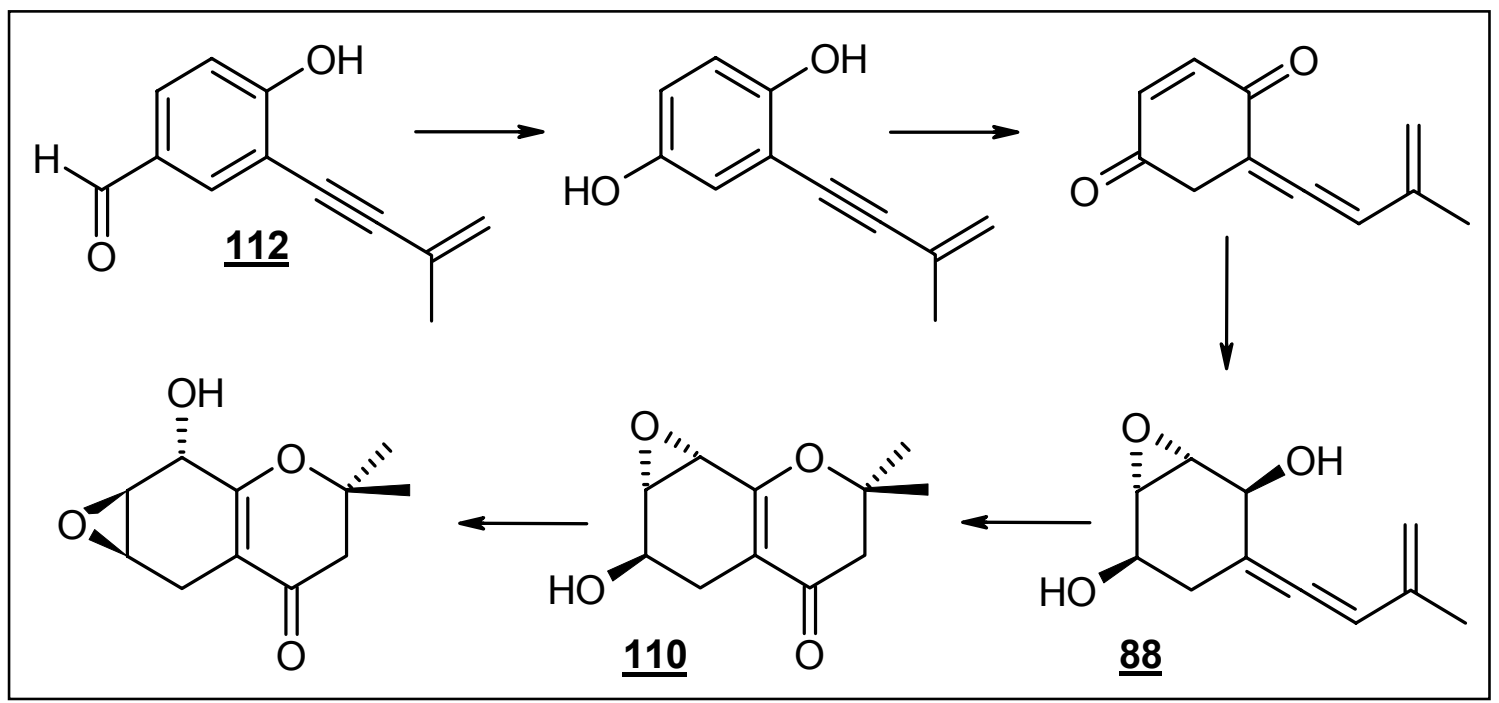

Abbildung 53: Postuliertes Biosyntheseschema für die Sekundärmetabolite aus Eutopia lata. ${ }^{132}$ 
Die Hypothese scheint für den Stamm WDMH46 unwahrscheinlich, da zum ersten kein Acetylen gefunden wurde, zum zweiten es so viele Allen-Derivate gab und zum dritten die Hauptmetaboliten allenischer Natur waren, wohingegen das Tetrahydrochromanon nur in sehr geringen Ausbeuten vorlag. Deshalb sollte durch Fütterungsexperimente mit isotopenmarkierten Verbindungen die biogenetische Herkunft der Strukturen, insbesondere der Allengruppierung, geklärt werden.

\subsubsection{Zur Biogenese der Allene aus WDMH46}

\subsubsection{Fermentationskurve}

Wie schon in Kapitel 5.4.2.1 beschrieben, ist die Kenntnis über den zeitlichen Produktionsverlauf eines Stammes unerlässlich, um Fütterungsexperimente durchzuführen. Zur Aufnahme einer Fermentationskurve wurde der Stamm WDHM46 über 9 Tage im Nährmedium 1405 kultiviert. Ab der 48. Stunde entnahm man alle 12 Stunden jeweils zwei Kolben und arbeitete sie nach gewohntem Schema (Abbildung 4) auf. Zwei weitere Kolben wurden über einen Zeitraum von 14 Tagen fermentiert. Die erhaltenen Kultufiltrat-Extrakte wurden dünnschichtchromatographisch untersucht. Die Sekundärstoffproduktion ergab sich wiederum aus der Intensität der UV-Löschung bei 254 nm und der Farbreaktion mit Anis.

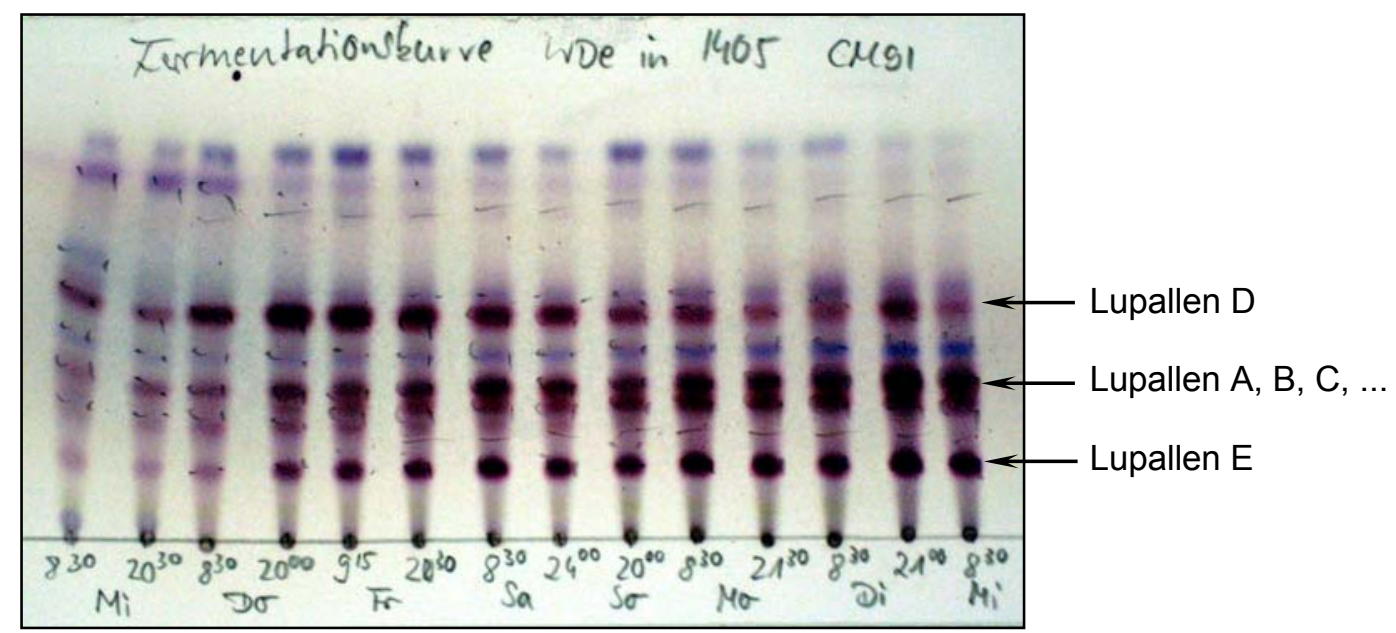

Abbildung 54: Fermentationskurve von WDMH46 auf DC-Kieselgel von der 48. bis 216 . Stunde. 
Es ist deutlich zu erkennen, dass die Allenproduktion bereits nach zweieinhalb Tagen einsetzt. Der erste gebildete Metabolit erweist sich als Lupallen D ( $\underline{\mathbf{9 0}})$, dessen Konzentration mit dem fünften Tag wieder abnimmt. Etwa zeitgleich zwischen der 72. und 83. Stunde beginnt die Bildung von Lupallen E ( $\underline{\mathbf{9 1}})$ und der mittelpolaren Allene $\underline{\mathbf{8 5}}, \underline{\mathbf{8 6}}$ und $\underline{\mathbf{8 7}}$, deren Menge steigt im Laufe der Fermentation an. Auch in den beiden Kolben der 14-tägigen Inkubation war kein Abbau dieser Metabolite zu verzeichnen. Da die Zufütterung markierter Substanzen mit dem Einsetzen der Produktion erfolgen sollte, um eine möglichst hohe IsotopenAnreicherung in den Molekülen zu erhalten, wurde die 60. Fermentationsstunde als optimaler Zeitpunkt für den Fütterungsbeginn ausgewählt.

\subsubsection{Fütterungsexperimente mit $\left[1-{ }^{13} \mathrm{C}\right]$ Acetat}

Der Stamm WDMH46 wurde in vier $1 \mathrm{~L}$-Erlenmeyerkolben ohne Schikanen mit jeweils $250 \mathrm{~mL} \mathrm{NL} 1405$ bei $28^{\circ} \mathrm{C}$ und $120 \mathrm{rpm}$ angezogen. Entsprechend der Ergebnisse aus der Fermentationskurve wurde die Precursor-Lösung (750 mg/L) ab der 60. Stunde im continous flow-Verfahren über 36 Stunden zugepumpt und die Fermentation nach der 120. Stunde abgebrochen. Nach der Aufarbeitung konnte nur Lupallen E (1) in einer Ausbeute von $2.9 \mathrm{mg} / \mathrm{L}$ isoliert werden. Ein ${ }^{13} \mathrm{C}$-Experiment zeigte nur eine leichte Intensitätserhöhung der Kohlenstoffatome C-7, C-9, C-1'und C-3'. Die Anreicherung lag unter einem Prozent. Als Erklärung könnte einerseits dienen, dass der Fütterungsbeginn für $\underline{91}$ nicht optimal getroffen wurde. Eine andere Möglichkeit ist, dass der Nachlauf mit 24 Stunden zu lang gewählt war und schon scrambling-Reaktionen eingesetzt hatten.

Aus diesem Grund wurde das Experiment unter veränderten zeitlichen Bedingungen wiederholt. Die Fütterung wurde mit der 62. Stunde begonnen und der Stamm nach wiederum 36 Stunden der Zugabe nur noch 12 Stunden weiter fermentiert. An die Aufarbeitung der Kulturbrühe schloss sich eine Säulenchromatographie des Rohproduktes an Sephadex LH-20 (Aceton) an. Zwei Fraktionen, deren Inhaltstoffe mit Anis eine pinke Farbreaktion gaben, wurden aufgefangen. Die Aufreinigung der polaren Komponente führte zu $1 \mathrm{mg}$ Lupallen E (91). Im ${ }^{13} \mathrm{C}$ NMR-Spektrum der $\left[1-{ }^{13} \mathrm{C}\right]$ Acetat angereicherten Substanz waren beachtliche Intensitätserhöhungen für C-7, C-9, C-1'und C-3' zu erkennen. Als Referenzsignal 
zur Berechnung des Einbaus diente das Kohlenstoffatom Nr. 8 der allenischen Methingruppe.

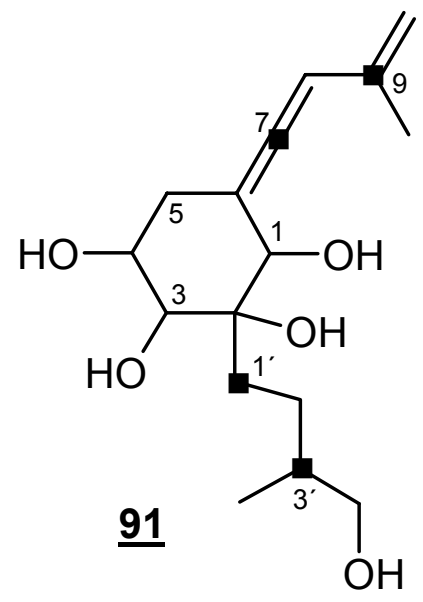

\begin{tabular}{|c|c|c|}
\hline $\mathrm{Nr}$. & ${ }^{13} \mathrm{C}-\mathrm{NMR}$ & spez. Einbau \\
\hline $1-\mathrm{CH}$ & 77.3 & 0.67 \\
\hline $2-\mathrm{C}$ & 74.8 & $-0,10$ \\
\hline $3-\mathrm{CH}$ & 77.2 & 0.36 \\
\hline $4-\mathrm{CH}$ & 68.6 & 0.21 \\
\hline $5-\mathrm{CH}_{2}$ & 30.5 & 0.46 \\
\hline $6-\mathrm{C}$ & 105.3 & -0.01 \\
\hline $\mathbf{7 - C}$ & $\mathbf{2 0 4 . 2}$ & $\mathbf{3 0 . 9 5}$ \\
\hline $8-\mathrm{CH}$ & 97.0 & 0 \\
\hline 9-C & $\mathbf{1 4 0 . 5}$ & $\mathbf{3 1 . 5 0}$ \\
\hline $10-\mathrm{CH}_{2}$ & 113.4 & 0.10 \\
\hline $11-\mathrm{CH}_{3}$ & 19.9 & 0.01 \\
\hline $\mathbf{1}^{\prime}-\mathrm{CH}_{2}$ & $\mathbf{3 4 . 6}$ & $\mathbf{3 6 . 8 9}$ \\
\hline $2^{\prime}-\mathrm{CH}_{2}$ & 26.6 & 0.05 \\
\hline $\mathbf{3}^{\prime}-\mathrm{CH}^{\prime}$ & $\mathbf{3 7 . 4}$ & $\mathbf{3 7 . 4 5}$ \\
\hline $4^{\prime}-\mathrm{CH}_{2}$ & 67.9 & 0.15 \\
\hline $5^{\prime}-\mathrm{CH}_{3}$ & 17.5 & 0,10 \\
\hline
\end{tabular}

Tabelle 14: Ergebnisse aus dem Fütterungsexperiment mit $\left[1-{ }^{13} \mathrm{C}\right]$ Acetat, spezifischer Einbau in Lupallen $\mathrm{E}(\underline{\mathbf{9 1}})$.

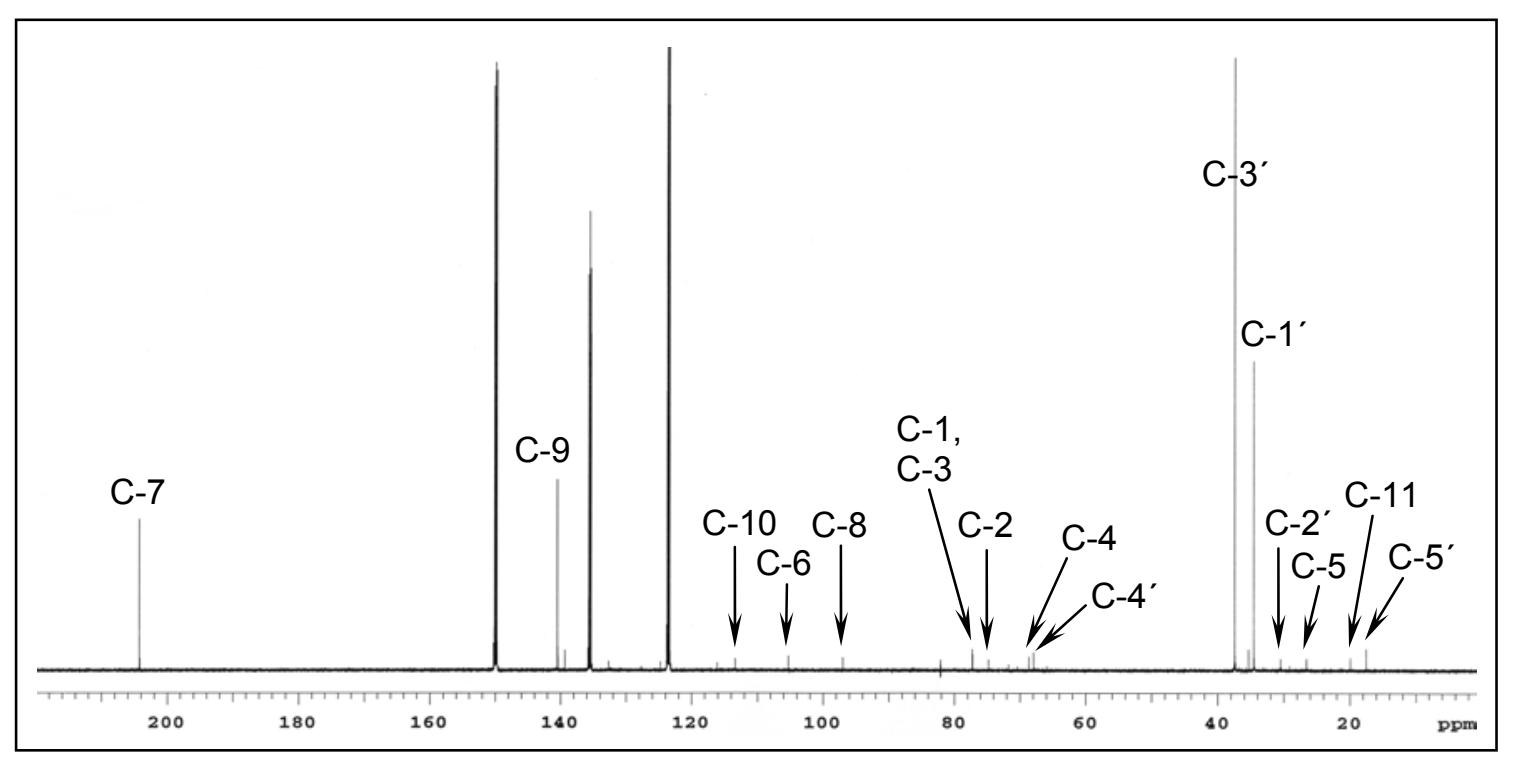

Abbildung 55: ${ }^{13} \mathrm{C}-\mathrm{NMR}-S p e k t r u m$ von Lupallen $\mathrm{E}(\underline{\mathbf{9 1}})$ nach Zufütterung von $\left[1-{ }^{13} \mathrm{C}\right] \mathrm{Acetat}$ (125 MHz, $\mathrm{D}_{5}$-Pyridin, $\left.35^{\circ} \mathrm{C}\right)$. 
Mit diesem Experiment konnte nicht nur die biogenetische Herkunft der beiden Cyclohexanseitenketten aus dem Acetat-Stoffwechsel, genauer dem IsoprenStoffwechsel, geklärt werden. Es war anhand des Einbaumusters (1,3-Markierung im IPP) ferner möglich, den Mevalonat-Weg als Biosyntheseroute zu bestimmen.

\subsubsection{Mevalonat- vs. Nicht-Mevalonat-Weg}

In der Vergangenheit wurde der Mevalonatweg als universelle Biosyntheseroute zu Isopentenylpyrophosphat (IPP), dem $\mathrm{C}_{5}$-Vorläufer der Terpene, angesehen. ${ }^{151}$ Die einzelnen Schritte wurden vor allem bei der Untersuchung der Steroid-Biosynthese in Lebergewebe und Hefe aufgeklärt. ${ }^{152,153}$ Zwei claisenähnliche Kondensationsreaktionen überführen Acetyl-CoA in Acetoacetyl-CoA und Hydroxymethylglutaryl-CoA (HMG-CoA). Eine NADPH-abhängige Reduktase katalysiert die Reduktion von HMG-CoA zu Mevalonat. Durch drei Phospho-rylierungen entsteht als Zwischenprodukt 3-Phospho-5-pyrophosphomevalonat. Die Phosphatgruppe an $\mathrm{C}-3-\mathrm{OH}$ fungiert als gute Abgangsgruppe und wird wie die benachbarte Carboxylgruppe im folgenden Schritt abgespalten, was zu einer Doppelbindung im $\mathrm{C}_{5}$-Produkt IPP führt. Ausgehend von $\left[1-{ }^{13} \mathrm{C}\right]$ Acetat entsteht eine 1,3-Markierung im IPP.

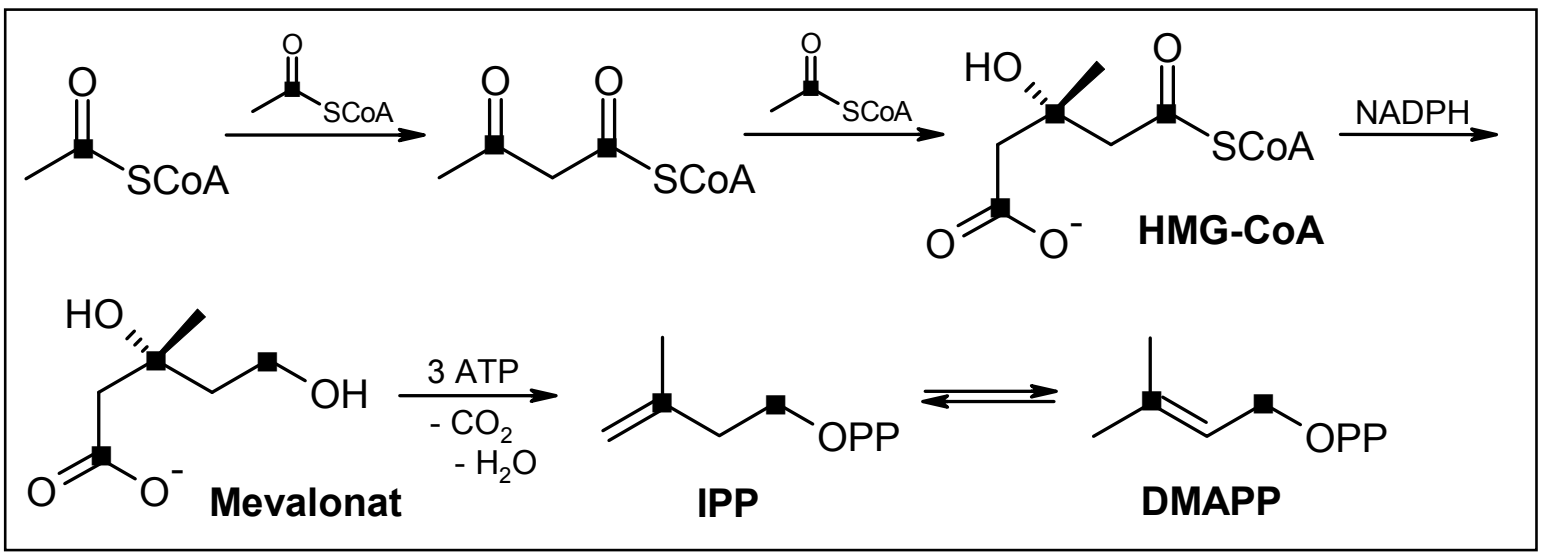

Abbildung 56: Der Acetat-Mevalonat-Weg der Isoprenoid-Biosynthese mit der Markierung von $\left[1{ }^{13} \mathrm{C}\right]$ Acetat.

Bei der Betrachtung des Acetat-Mevalonat-Weges als einzigen Ausgangspunkt des Terpenstoffwechsels gab es in der Vergangenheit oft widersprüchliche Ergeb- 
nisse bei Fütterungsexperimenten. Gerade in pflanzlichen Systemen wurden mit radioaktiv markierter Mevalonsäure nur geringe Einbauraten in Terpenen erzielt. $^{154,155}$ Darüber hinaus konnten einige Enzyme des Mevalonat-Weges in Chloroplasten nicht nachgewiesen werden. ${ }^{156} 1993$ wurde erstmals ein hypothetisches Schema des Triose-Pyruvat-Weges publiziert. ${ }^{157}$ Ausgangspunkt der Arbeiten waren Biosyntheseuntersuchungen an Zymomonas mobilis und E. coli. ${ }^{158}$ Die Stämme wurden in Nährmedien mit ${ }^{13} \mathrm{C}$-markierter Glucose kultiviert. Die isolierten Hopanoide zeigten ein Markierungsmuster, das nur über einen Mevalonat-unabhängigen Mechanismus erklärt werden konnte. ${ }^{159}$ Etwa zur gleichen Zeit beschäftigte sich die Arbeitsgruppe von D. ARIGONI mit der Biosynthese terpenoider Verbindungen aus Ginkgo biloba. Die Markierungsmuster der untersuchten Ginkgolide ${ }^{160}$ entsprachen dem vorgeschlagenen Triose-Pyruvat-Weg.

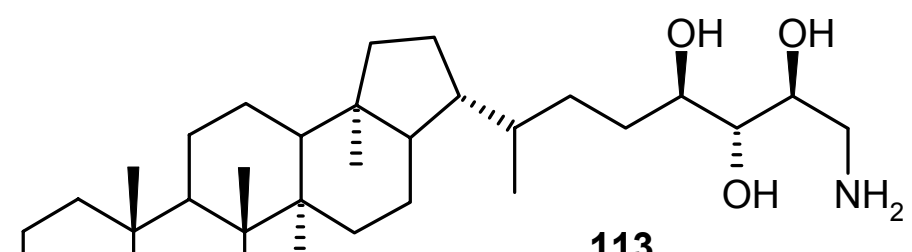

$\underline{113}$

Hopanoid

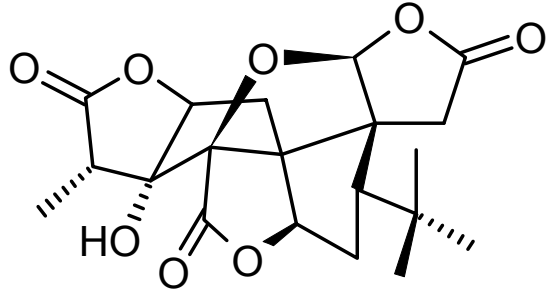

114

Ginkgolid A

Charakteristisch für den Triose-Pyruvat-Weg ist die Bildung von 1-Desoxy-D-xylulose-5-phosphat (DXP), das durch Kondensation von Glycerinaldehyd-3-phosphat (GAP) und Pyruvat entsteht. Eine nachfolgende Umlagerungsreaktion führt über das Intermediat 2-Methylerythrose-4-phosphat zu 2-Methyl-D-erythritol-4-phosphat (MEP). Die abschließende Reaktion zu IPP kann mechanistisch noch nicht eindeutig formuliert werden. ${ }^{161}$ Ausgehend von $\left[1-{ }^{13} \mathrm{C}\right]$ Acetat ist IPP nur an C-4 markiert (Abbildung 57).

Die Existenz dieses Weges wurde bislang in Pflanzen, Grünalgen und Bakterien bewiesen. ${ }^{162}$ In Pflanzen existieren beide Wege nebeneinander. Es wird vermutet, dass in den Chloroplasten der Triose-Pyruvat-Weg abläuft (Bindung von Phytol, Carotinoiden,...) und im Cytosol der Acetat-Mevalonat-Weg bevorzugt wird. Möglicherweise findet ein Transfer von Prenyldiphosphaten zwischen dem Cytosol und den Chloroplasten statt. ${ }^{162}$ 


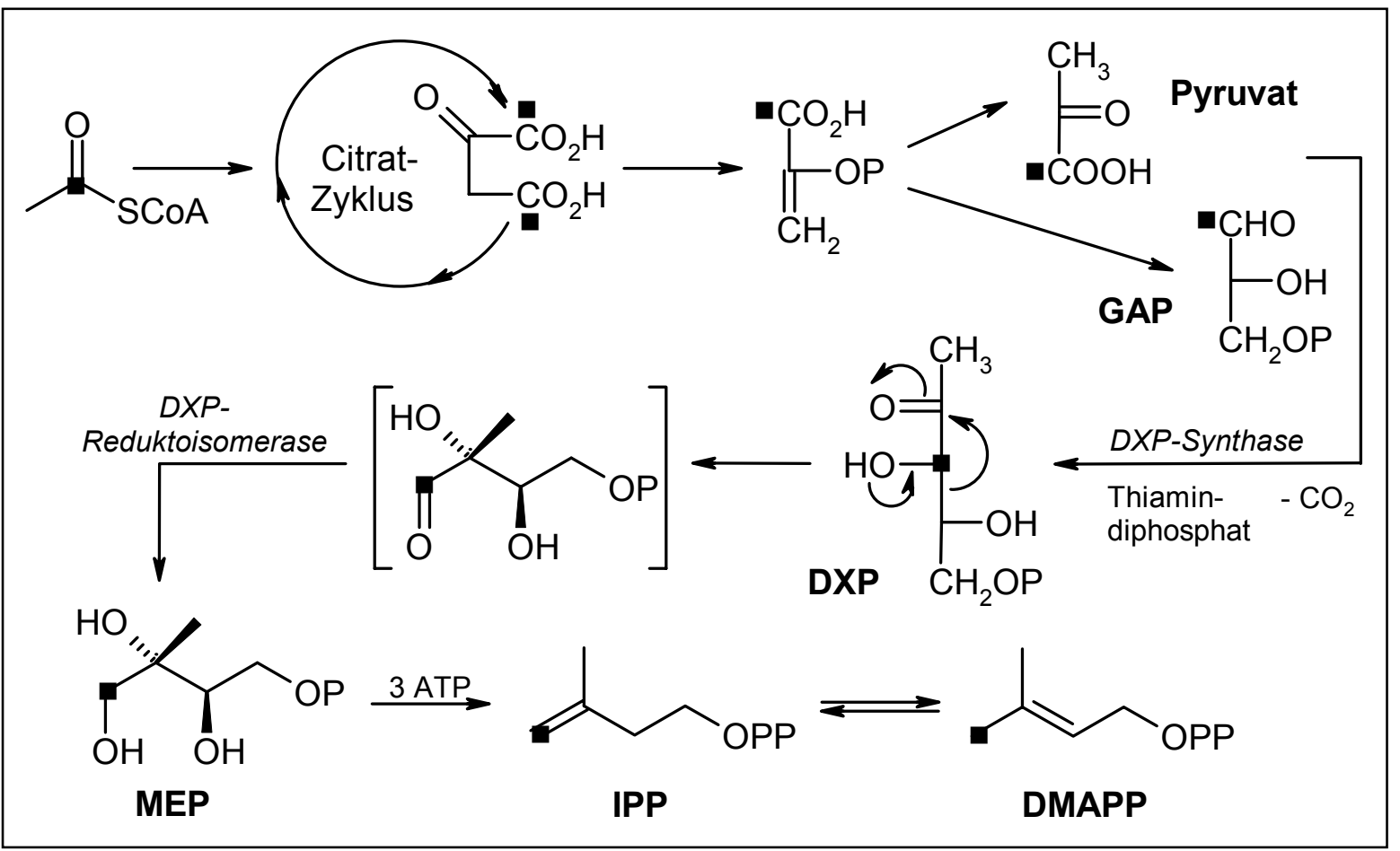

Abbildung 57: Hypothetischer Isoprenoid-Biosyntheseweg aus Glycerinaldehyd-3-phosphat und Pyruvat mit der Markierung von $\left[1{ }^{13} \mathrm{C}\right]$ Acetat.

\subsubsection{Fütterungsexperimente mit $\left[1,2,3-{ }^{13} \mathrm{C}_{3}\right] \mathrm{Glycerin}$}

Die Seitenketten stammen, wie der Acetateinbau zeigte, aus dem Isopren-Stoffwechsel. Im Cyclohexanring konnte keine Isotopenanreicherung nachgewiesen werden. Demzufolge muss dieser Strukturteil aus einem anderen Biosynthesepool, möglicherweise dem Shikimat-Stoffwechsel, gespeist werden.

Man fermentierte den Stamm WDMH46 analog zur Acetatfütterung in vier $1 \mathrm{~L}$ Erlenmeyerkolben ohne Schikanen mit jeweils $250 \mathrm{~mL} \mathrm{NL} 1405$ bei $28^{\circ} \mathrm{C}$ und $120 \mathrm{spm}$. Von der 53. bis zur 89. Stunde wurde die Glycerin-Lösung $(750 \mathrm{mg} / \mathrm{L}) \mathrm{im}$ continous flow-Verfahren zugepumpt und die Fermentation nach weiteren sieben Stunden abgebrochen. Nach der Standardaufarbeitung konnte im Rohprodukt keine mit Anis pink anfärbende Substanz detektiert werden. Durch Säulenchromatographie an Kieselgel und Sephadex LH-20 (Methanol) erhielt man 2.6 mg 3-Dimethylallyl-4-hydroxybenzoesäure ( $\underline{96})$. 


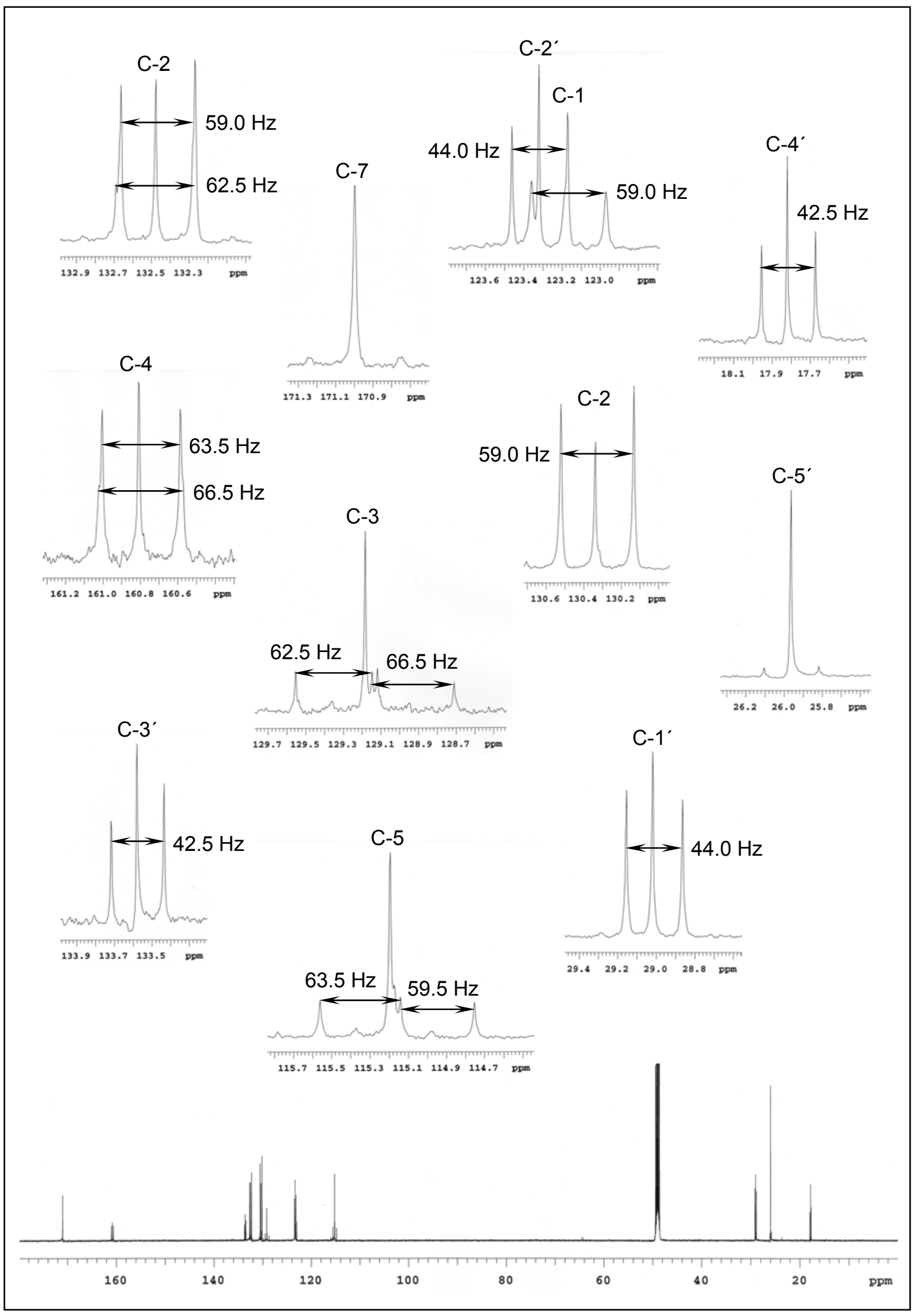

Abbildung 58: ${ }^{13} \mathrm{C}$-Spektrum $\left(125 \mathrm{MHz}, \mathrm{D}_{5}\right.$-Pyridin, $\left.35^{\circ} \mathrm{C}\right)$ von $\underline{96}$ mit der Markierung von $\left[1,2,3-{ }^{13} \mathrm{C}_{3}\right]$ Glycerin inklusive Spreizungen. 
Von den 12 Kohlenstoffatomen waren im ${ }^{13} \mathrm{C}-\mathrm{NMR}$-Spektrum nur zwei als Singulett-Signale zu beobachten, eine Seitenketten-Methylgruppe $\left(\delta_{C}=26.0\right)$ und das Signal des Carbonsäurezentrums bei $\delta_{\mathrm{C}}=171.0$. Bei den anderen Signalen wurden neben den typischen Kopplungen aus uniform markiertem Glycerin auch andere Multiplizitäten aufgefunden, die so nicht vorherzusehen waren.

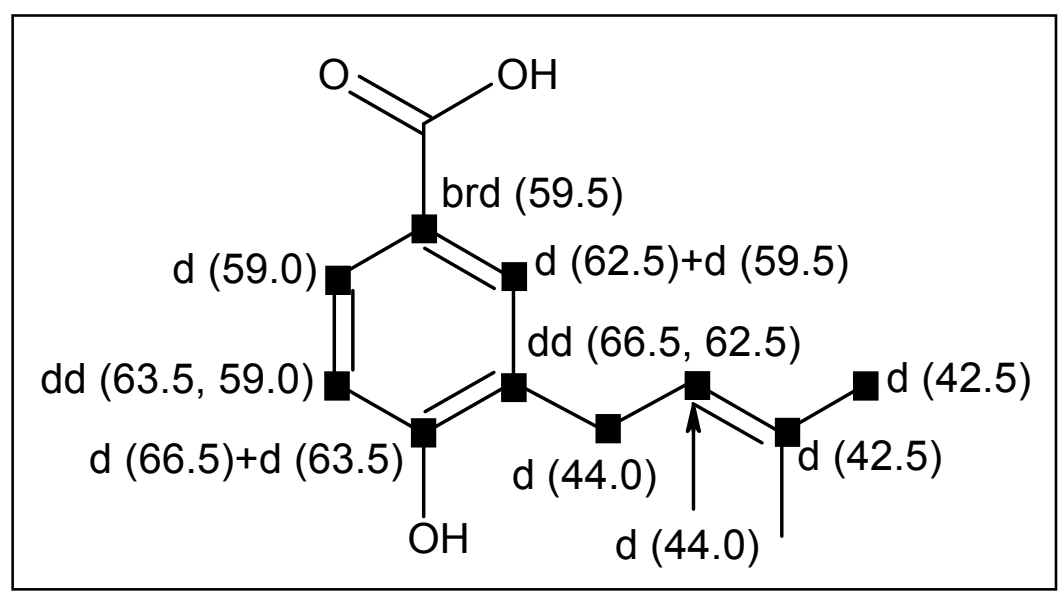

Abbildung 59: Aus dem ${ }^{13} \mathrm{C}-\mathrm{NMR}-S p e k t r u m$ von $\underline{96}$ ableitbares Kopplungsmuster nach der Fütterung von uniform markiertem Glycerin.

Vergleicht man die Beobachtungen mit einem erwarteten Glycerineinbau, kommt man zu dem Ergebnis, dass der Metabolit über eine symmetrische Zwischenstufe, wie z.B. para-Hydroxybenzoesäure, aufgebaut wird. Anhand der Abbildung 60 lässt sich diese Theorie bestätigen. In der Isoprenylseitenkette konnte außerdem ein Einbau von zwei intakten Acetateinheiten nachgewiesen werden.

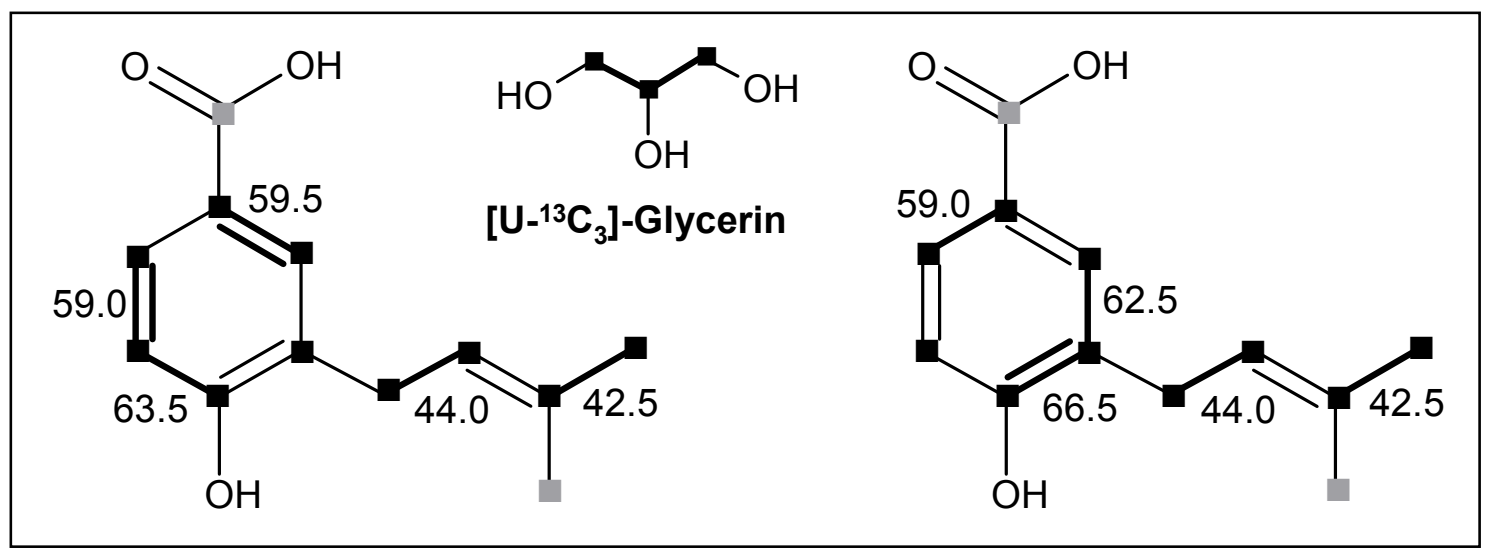

Abbildung 60: Über eine symmetrische Zwischenstufe erklärbare Kopplungsmuster der Kohlenstoffsignale von $\underline{96}$ nach der Fütterung von uniform markiertem Glycerin. 
Die Bestimmung der Anreicherung für C-1 und C-5' war wegen fehlender Referenzatome nicht möglich. Für 3-Dimethylallyl-4-hydroxybenzoesäure (흐) kann aus dem Fütterungsexperiment folgendes hypothetisches Biosyntheseschema aufgestellt werden. Shikimat wird über Chorismat und Tyrosin in p-Hydroxybenzoesäure (PHB) umgewandelt. Die anschließende Addition von IPP, gebildet über den Acetat-Mevalonat-Weg, liefert $\underline{96}$. Die Biosynthese erinnert an die Bildung der Pflanzenmetaboliten Alkannin (115) und Shikonin (116), auf die im nächsten Kapitel kurz eingegangen werden soll.

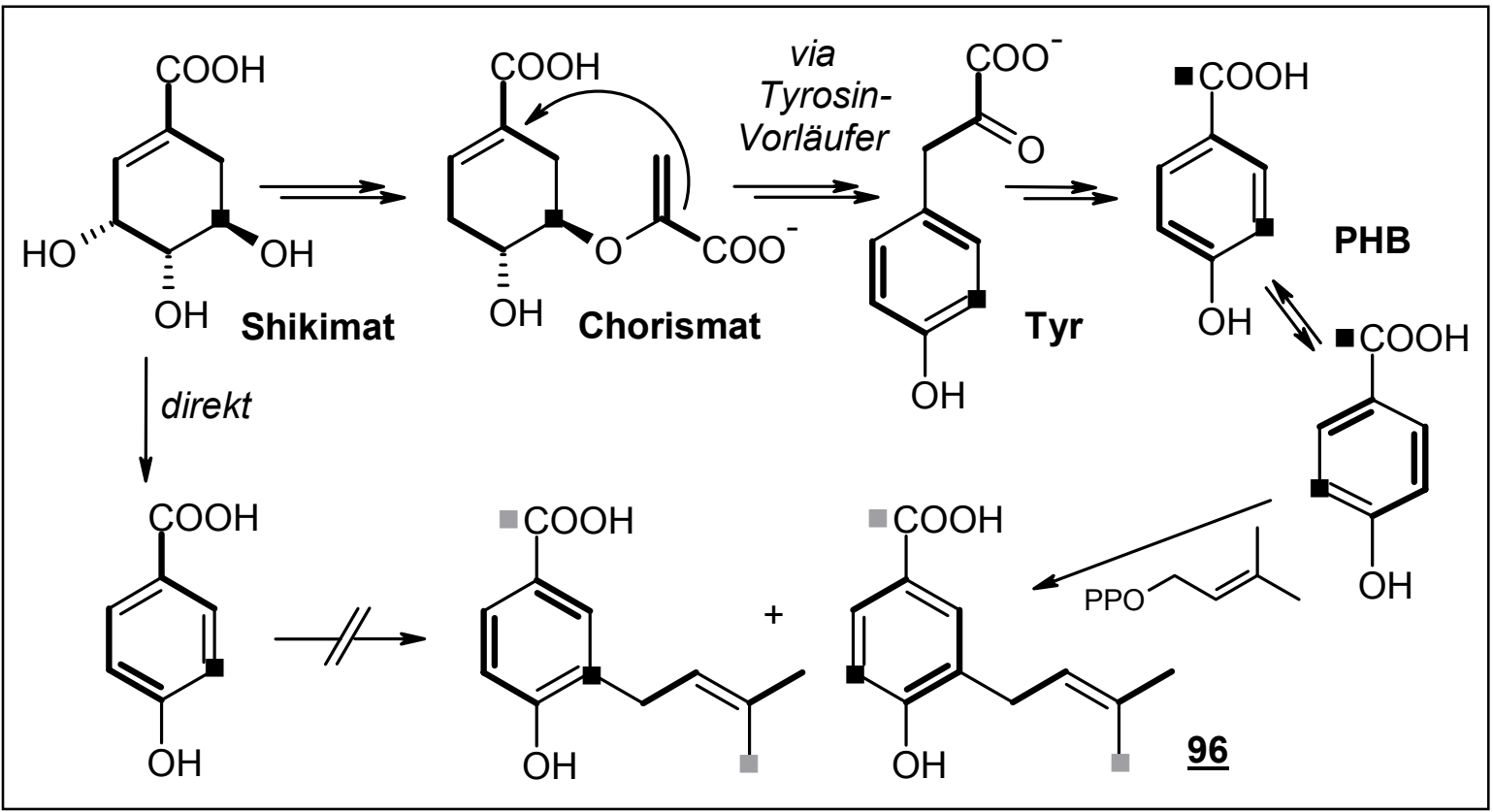

Abbildung 61: Hypothetische Biosynthese für die Bildung von $\underline{96}$ mit der Markierung von $\left[\mathrm{U}^{13} \mathrm{C}_{3}\right]$-Glycerin.

\subsubsection{Zur Biosynthese von Alkannin und Shikonin}

Alkannin $(\underline{\mathbf{1 1 5}})$ und Shikonin ( $\underline{\mathbf{1 1 6}})$ wurden erstmals 1935 aus den Extrakten der Wurzeln Alkanna tinctoria (Europa) und Lithospermum erythrorhizon (Asien) isoliert und als Enantiomere identifiziert. ${ }^{163}$ Die Extrakte der Wurzeln sind schon seit Jahrhunderten als rote Naturfarbstoffe und<smiles>CC(C)=CC[C@@H](O)C1=CC(=O)c2c(O)ccc(O)c2C1=O</smiles>

Alkannin (115) 
Wundheilungsmittel verwendet worden. ${ }^{164} 1976$ wurde die wundheilende Wirkung von Alkannin durch klinische Studien belegt. ${ }^{165}$ Vor kurzem konnte für beide Verbindungen neben der antibakteriellen und entzündungshemmenden Wirkung auch Antitumor-Eigenschaften nachgewiesen werden. ${ }^{164}$

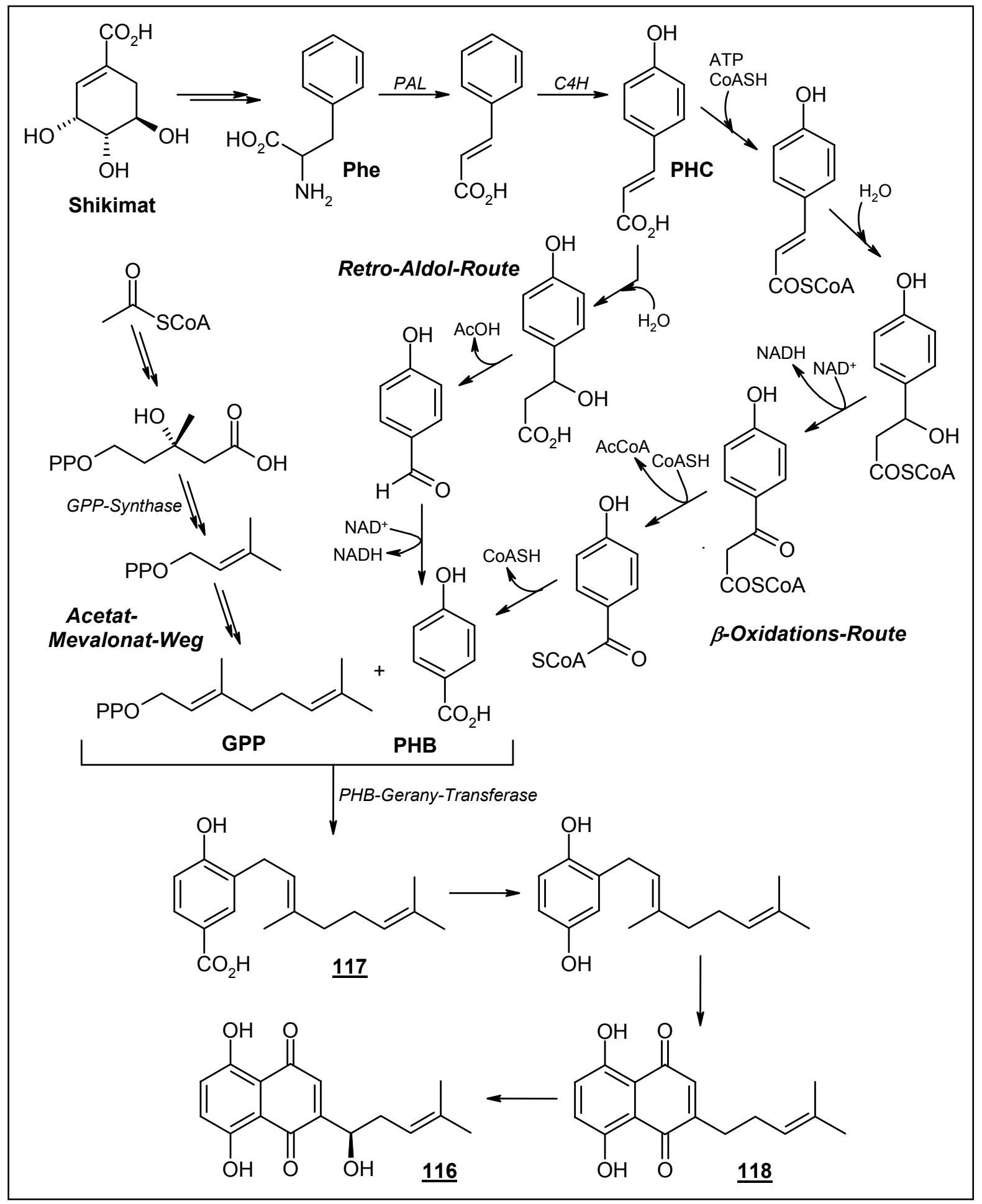

Abbildung 62: Biosynthese von Shikonin (116) ${ }^{164}$ 
Durch die intensive Erforschung der kommerziellen Produktion von $\underline{115}$ und $\underline{116}$ in pflanzlichen Zellkulturen wurde die Aufklärung verschiedener Stadien der Biosynthese dieser Naturstoffe stark vorangetrieben. ${ }^{166}$ Von den frühen Biosynthesestufen, der Bildung der beiden Hauptvorläufer 4-Hydroxybenzoesäure (PHB) und Geranylpyrophosphat (GPP), ist wesentlich mehr bekannt als von den späteren. ${ }^{165}$ GPP ensteht auf der gut untersuchten Isoprenoid-Route ${ }^{167}$ über Mevalonat als Zwischenstufe, während die Synthese von PHB ihren Ausgangspunkt in Shikimisäure findet. Aus dieser entsteht in mehreren Stufen L-Phenylalanin (Phe), welches durch Phenylalanin-Ammoniak-Lyase $(P A L)$ zu Zimtsäure abgebaut wird. Deren weitere Umwandlung, katalysiert durch Cinnamat-4-Hydroxylase $(\mathrm{C} 4 \mathrm{H})$ liefert das Zwischenprodukt $p$-Hydroxyzimtsäure (PHC). Es bestehen nun unterschiedliche Auffassungen darüber, ob die biosynthetische Transformation von PHC zu PHB auf der $\beta$-Oxidations-Route ${ }^{168}$ oder über einen Retro-AldolMechanismus $^{169}$ verläuft. Die beiden Precurser PHB und GPP werden, katalysiert durch das Enzym PHB-Geranyl-Transferase, zu 3-Geranyl-PHB (117) umgesetzt und in nachfolgenden Schritten über 3-Geranylhydrochinon (118) in Shikonin (116) bzw. Alkannin (115) überführt. Über vielfache Fütterungsexperimente konnte bewiesen werden, dass neben Shikimisäure auch L-Phenylalanin, Zimtsäure, PHB, 3-Geranylhydrochinon und Mevalonsäure Zwischenstufen dieser Biosynthese sind und Shikonin (116) tatsächlich auf dieser biosynthetischen Route entsteht. ${ }^{170}$ In einer Publikation von C. HERTWECK und B.S. MOORE aus dem Jahr 2000 wird von einer Benzoyl-CoA-Biosynthese in dem marinen Bakterium Streptomyces maritimus berichtet, ${ }^{171}$ die analog $z u$ denen in Pflanzen abläuft. Fütterungsexperimente mit ${ }^{2} \mathrm{H}$ - und ${ }^{13} \mathrm{C}$-markierten Intermediaten konnten zeigen, dass Phenylalanin zu Zimtsäure metabolisiert und schließlich durch $\beta$-Oxidation in BenzoylCoA überführt wird. Benzoyl-CoA dient als Startereinheit für eine Typ II- Polyketidsynthase zur Produktion der bakteriostatischen Polyketide Enterocin (119) und der Wailupemycine. Die Ergebnisse der Fütterungsstudien stehen im Einklang mit dem hypothetischen Biosynthesemodel, das aus dem klonierten EnterocinBiosynthesegencluster abgeleitet wurde. ${ }^{171}$ 


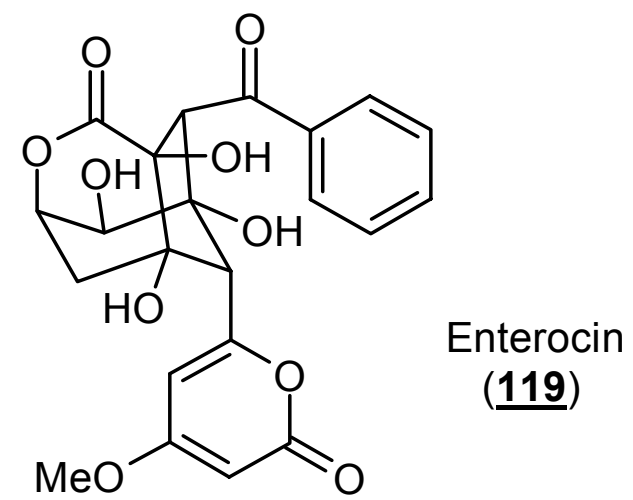

\subsubsection{Zufütterung potentieller Biosynthesevorläufer}

In Anlehnung an das letzte Kapitel sollte durch Fütterung unmarkierter potentieller Biosynthesevorläufer geprüft werden, ob dadurch eine Ausbeutesteigerung der Sekundärmetabolite des Stammes WDMH46 erreicht werden kann.

Der Stamm wurde analog zu den anderen Fütterungen im 1 L-Ansatz (NL 1405) angezogen. Von der 60. bis zur 108. Stunde wurde alle 12 Stunden jeweils $1 \mathrm{~mL}$ der Substrat-Lösung (sechs Portionen) der wachsenden Kultur zugefügt. 24 Stunden später wurde der Ansatz geerntet und wie gewohnt aufgearbeitet. Die Kulturfiltrat-Extrakte wurden an der HPLC analysiert.

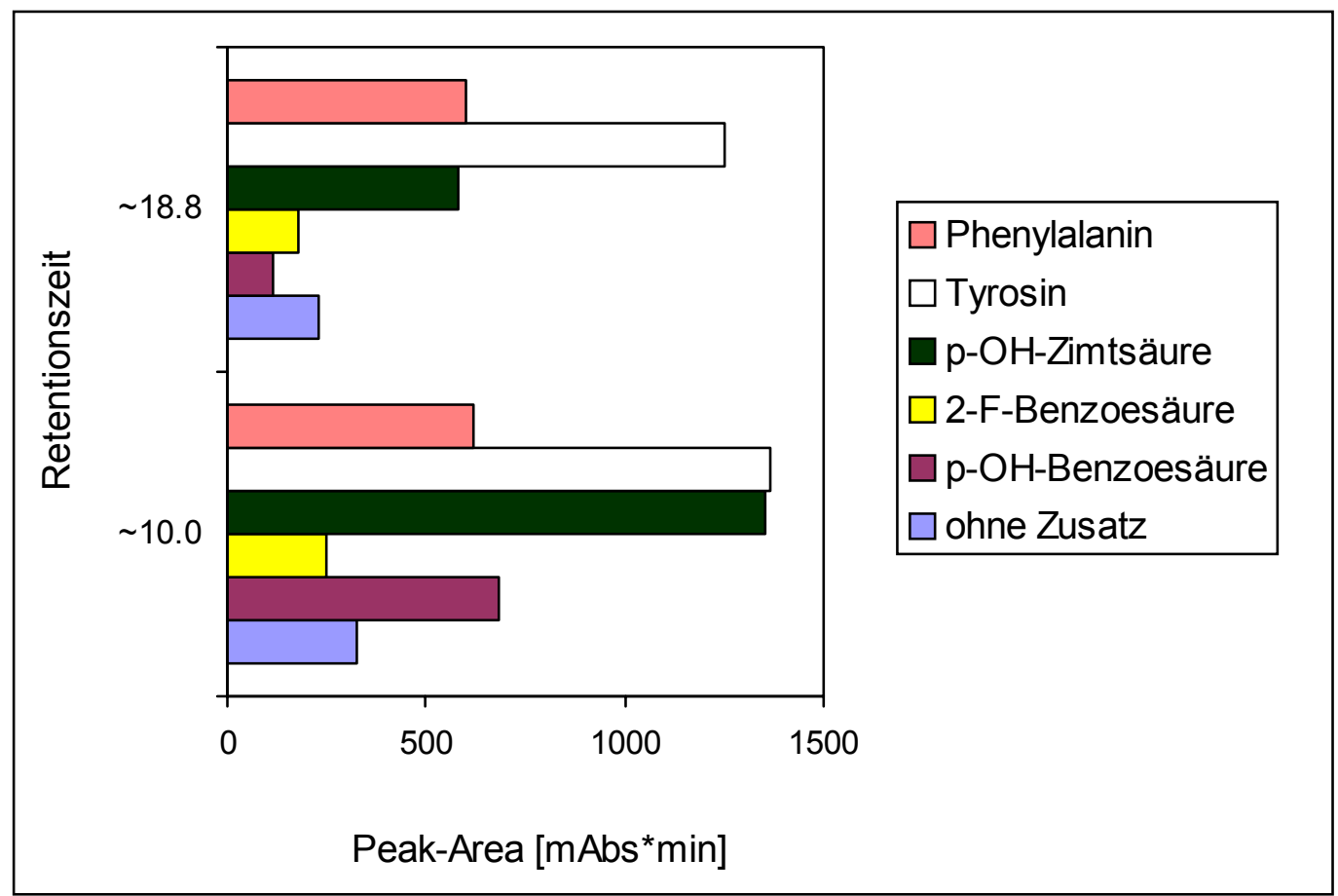

Abbildung 63: HPLC-Analyse bezüglich der Lupallene $E\left(R_{t}=10.0\right)$ und $C\left(R_{t}=18.8\right)$ nach der Zufütterung potentieller Vorläufer. 
Eine Auswertung der HPLC-Chromatogramme ergab, dass sich die Zugabe von $p$ Hydroxybenzoesäure ( $5 \mathrm{mmol} / \mathrm{L}), p$-Hydroxyzimtsäure, Tyrosin und Phenylalanin ( $2 \mathrm{mmol} / \mathrm{L})$ positiv auf die Produktion der Lupallene auswirkt. Die Ausbeute an Lupallen E (91) konnte durch PHB und Phe verdoppelt, mit PHC und Tyrosin sogar vervierfacht werden. Die Produktionssteigerungen sprechen dafür, dass die gefütterten Substanzen möglicherweise Intermediate auf dem Weg zu den Lupallenen sind. Lediglich die Zugabe von 2-Fluorbenzoesäure führte zu einer

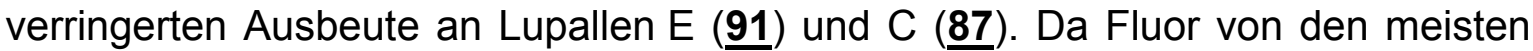
Enzymen als Wasserstoffersatz akzeptiert wird, scheint die fehlende Hydroxygruppe in para-Stellung der entscheidene Parameter für den Rückgang der Allenbildung zu sein.

\subsubsection{Zur Biosynthese der WDMH46-Metabolite}

Die isolierten Verbindungen und die beschriebenen Fütterungsexperimente erlauben einige Rückschlüsse auf die Lupallen-Biosynthese und führen zu dem hypothetischen Biosytheseschema in Abbildung 64.

Die beiden Hauptvorstufen sind p-Hydroxybenzoesäure (PHB) und IsopentenylPyrophosphat (IPP). Ausgehend von Shikimat wird über Phenylalanin oder Tyrosin PHB gebildet, während IPP bzw. DMAPP über die Mevalonat-Route aus Acetat entsteht. Anschließend werden beide Verbindungen zu 3-Isopentenyl-PHB vernüpft. Durch Wasseraddition und Dehydrierung kann die Bildung von $\underline{97}$ erklärt werden. Dieser Metabolit scheint für weitere Schritte in Richtung Allene desaktiviert zu sein, da keine weitere Substanz mit gleicher Seitenkette isoliert wurde. Weil PHB ein symmetrisches Substrat darstellt, wird die Addition eines zweiten IPP- bzw. DMAPP-Restes, ebenfalls in meta-Position zur Carboxylgruppe, angenommen, woraus nach oxidativer Decarboxylierung das symmetrische Chinon $\underline{120}$ entsteht. Die Epoxidierung des Chinons macht das Molekül chiral und erlaubt eine enzymatische Unterscheidung der beiden Seitenketten. Während die eine nur isomerisiert, wird in die andere eine Ketogruppe hineinoxidiert. Nach Reduktion der C-1 Carbonylgruppe gelangt man zur Zwischenstufe 121. Die Addition von Wasser in die eine Seitenkette und Cyclisierung des $\alpha, \beta$-ungesättigten Ketons der anderen mit der Hydroxygruppe im Ring führt zum Tetrahydrochromanon-Derivat $\underline{95}$. 


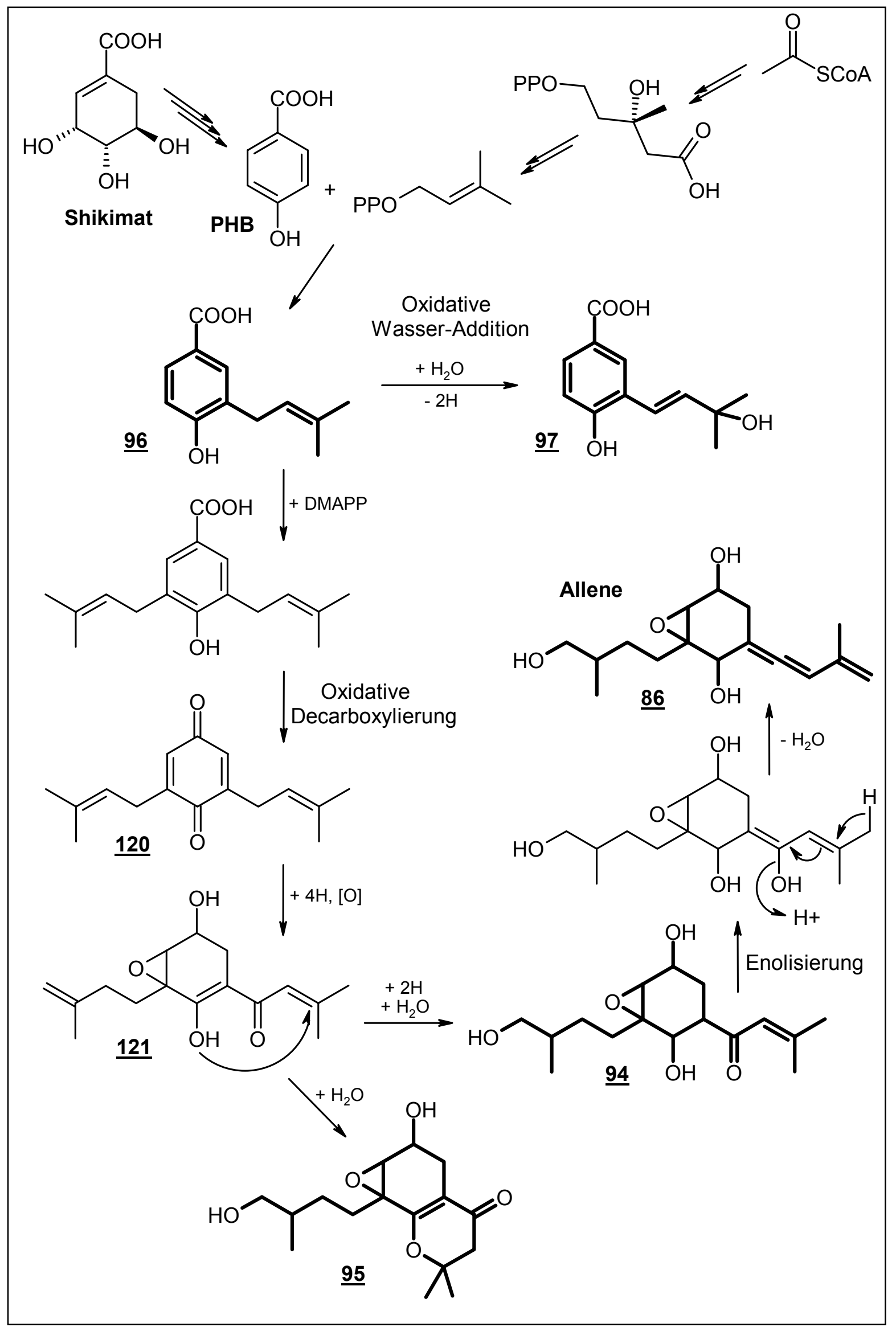

Abbildung 64: Hypothetisches Biosyntheseschema für die Metaboliten aus WDMH46. 
Entgegen der Hypothese von R. TABACCHI und seinen Mitarbeitern, die das exocyclische Allen $\underline{88}$ aus Eutopia lata als Biosyntheseintermediat zum strukturverwandten Tetrahydrochomanon gesehen haben (Kapitel 6.7.1), ${ }^{132}$ wird das bicyclische System $\underline{95}$ als Shunt-Produkt der Lupallen-Biosynthese eingeordnet. Wird neben der Wasser-Addition in der linken Seitenkette anstelle der Cyclisierung eine Reduktion im Sechsring postuliert, kommt man zum Intermediat $\underline{\mathbf{9 4}}$, das aus dem Allenproduzenten isoliert worden war. Eine Enolisierung, gefolgt von einer 1,4-Wassereleminierung eröffnet den Weg zum Allen, entsprechend Lupallen A (흐). In der späten Biosynthese entstehen aus $\underline{\mathbf{8 6}}$ die anderen Lupallene.

\subsection{Zur biologischen Aktivität der Lupallene}

Von den isolierten Verbindungen wurden die Lupallene $A, C$ und $E(\underline{86}, \underline{\mathbf{8 7}}, \underline{91})$ auf ihre biologische Aktivität im Plattendiffusionstest gegen die Testkeime Candida albicans, Stapphylococcus aureus, Bacillus subtilis und Escherichia coli getestet. Bei keiner der Verbindungen war auf den Testplatten ein Hemmhof zu beobachten.

Zusätzlich wurden die Metabolite in die Testsysteme der BASF eingeschleust. Der Fungizid-Test lieferte keinen Treffer, die anderen Ergebnisse stehen noch aus.

Zur Untersuchung der Metabolite auf mögliche Antitumoreigenschaften erfolgten durch Prof. Dr. BeIL (Medizinische Hochschule Hannover). Gegen die dort verwendeten Tumorzelllinien (Magenadenocarcinom HM02, Lebercarcinom HepG2, Mammacarcinom MCF7) zeigten die Lupallene keine Wirkung.

$\mathrm{Da}$ die Allengruppierung als Suizid-Substrat enzymatischer Reaktionen gilt (Kapitel 6.1), wurden die Naturstoffe zwei Arbeitsgruppen zugeführt, um auf Antimalaria-Aktivität bzw. Phosphatase-Aktivität zu prüfen. Auch hier stehen die Testergebnisse noch aus. 


\section{Zusammenfassung der Ergebnisse}

Im Rahmen dieser Arbeit wurden 12 Pilzstämme aus marinen Habitaten, vorwiegend der Nordsee, in einem chemischen Screening auf ihre Sekundärstoffproduktion hin untersucht.

Isolate aus dem marinen Lebensraum konnten ihr großes Potential bezüglich der Diversität an Sekundärmetaboliten bereits oft unter Beweis stellen und ließen auf ungewöhnliche Biosynthesewege und interessante neue Strukturen hoffen.

Sechs im chemischen Screening auffällige Stämme wurden einer intensiveren Bearbeitung unterzogen, um die Pilzmetabolite zu identifizieren. Insgesamt konnten 27 Naturstoffe in ihrer Struktur bestimmt werden, 16 davon waren neu.

\section{WDMH35 (Aspergillus fumigatus)}

Im Myzel-Extrakt dieses Stammes ließ sich das bekannte Diketopiperazin Fumitremorgin $C(\underline{\mathbf{3 2}})$ nachweisen. Aus dem Kulturfiltrat konnten neben $\underline{32}$ die Spiroverbindung cis-Pseurotin (29) und Fumigaclavin A ( $\underline{\mathbf{3 1}})$ identifiziert werden.

Diese Verbindungen sind aus anderen Stämmen gleicher Gattung bekannt. Um unnötige Reisolierungen zu vermeiden, wurden die Extrakte von WDMH35 einer HPLC-MS-Analyse unterzogen. Die Ergebnisse deuten darauf hin, dass nur die bekannten Metabolite aus Aspergillus fumigatus gebildet worden waren. Auf eine weitere Bearbeitung wurde verzichtet.

\section{WDMHC2 (Fusarium sp.)}

Der Hauptmetabolit (Nährmedium 1158) war das literaturbekannte Cyclonerodiol (43), ein Sesquiterpendiol.

\section{WDMH24 (Penicillium coralligerum)}

Dieser Stamm lieferte als einziges Hauptprodukt das $\mathrm{N}$-(2-cis(4-Hydroxyphenyl)ethenyl)-formamid (44). 44 wurde in der Literatur zuvor als Metabolit von Aspergillus fumigatus beschrieben. 


\section{WDMH51 (Aspergillus sp.)}

Der Stamm produzierte mehrere Verbindungen, von denen vier aufgereinigt und

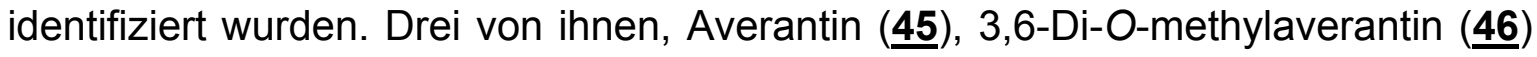
und 6,8-Di-O-methylnidurufin (47), sind Anthrachinone, während das Sterigmatocystin ( $\underline{58})$ eine Xanthonstruktur ausweist. $\underline{46}$ ist als Naturstoff bisher nicht beschrieben. Der biogenetische Zusammenhang der Sekundärmetabolite wird beschrieben. Alle Substanzen sind Biosynthesestufen des Aflatoxins (모).

Aufgrund der karzinogenen, teratogenen, hepatotoxischen und immunsupressiven Wirkung von $\underline{49}$ wurde von einer weiteren Bearbeitung des Stammes Abstand genommen.

\section{WDMH28 (nicht identifiziert)}

Aus diesem Pilzstamm konnten aus den Rohprodukten durch mehrere Trennschritte sechs Substanzen rein erhalten werden.

1) Neben dem typischen Pilzmetaboliten Isoscleron (20) wurde sein 6-MethoxyDerivat $\underline{\mathbf{5 2}}$ und $\alpha$-Acetylorcinol ( $\underline{\mathbf{5 0}})$ identifiziert. $\underline{\mathbf{5 2}}$ war mit diesem Substitutionsmuster bisher nicht beschrieben.

Ferner wurde erstmals 6-Methoxyparvulenon ( $\underline{56})$ isoliert und charakterisiert. Für die Strukturaufklärung erwiesen sich die Acetate $\underline{\mathbf{5 4}}$ und $\underline{55}$ als hilfreich.

Die Analyse der NMR-Daten führte zur Struktur der interessanten neuen Verbin-

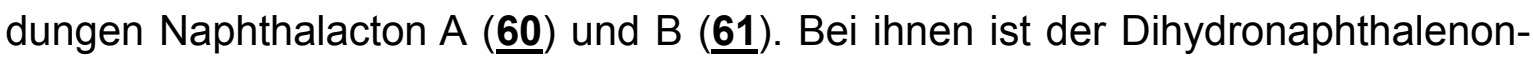
Grundkörper durch einen Lactonring erweitert.<smiles>CC(=O)Cc1cc(O)cc(O)c1</smiles>

$\underline{50}$<smiles>COc1cc2c(c(O)c1C(C)O)C(=O)CCC2O</smiles><smiles>O=C1CC[C@@H](O)c2cccc(O)c21</smiles>

$\underline{51}$<smiles>COc1cc(O)c2c(c1)[C@H](O)CCC2=O</smiles>

$\underline{52}$<smiles>COc1cc2c(c(O)c1C(C)O)C(=O)CCC21CC(O)C(=O)O1</smiles>

$\underline{60}$ 
2) Durch Fütterungsexperimente mit $\left[1-{ }^{13} \mathrm{C}\right] \mathrm{Acetat},\left[1,2-{ }^{13} \mathrm{C} 2\right]$ Acetat und $\left[2-{ }^{13} \mathrm{C}\right]-$ Malonsäure konnte bewiesen werden, dass das Parvulenon-Gerüst in $\underline{\mathbf{5}} \underline{\mathbf{6}}$ und $\underline{\mathbf{6 0}}$ auf dem Polyketidweg entsteht. Die Einbaumuster im $\gamma$-Lactonring von $\underline{\mathbf{6 0}}$ entsprechen denen für Marticin (4) und Canescin (드) und belegen, dass der 5-Ring aus einer $\mathrm{C}_{3}$-Einheit gebildet wird, die aus dem Citratcyclus stammt. Diese Ergebnisse konnten durch den erfolgreichen Einbau von $\left[1,4-{ }^{13} \mathrm{C}_{2}\right]$ Succinat untermauert werden.

Ein Biosyntheseschema für Naphthalacton A $(\underline{\mathbf{6 0}})$ wird formuliert.

3) Für keinen der Metabolite ist bislang eine biologische Aktivität gefunden worden.

\section{WDMH46 (Paraspheaoshaeria sp.)}

Dieser Stamm produzierte mehrere Sekundärmetabolite mit Allen-Struktur. Aus den Extrakten eines 5 L-Ansatzes in Schüttelkolben (NL 1158) wurden die

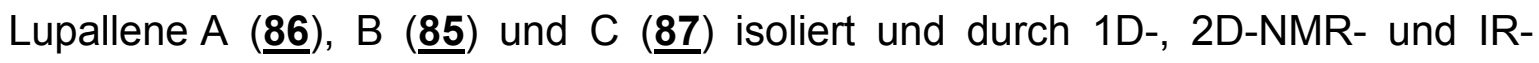
Spektroskopie in ihrer Struktur aufgeklärt. Wegen der seltenen Allengruppierung schlossen sich ausführliche Untersuchungen bezüglich des Einflusses ausgewählter Fermentationsparameter auf die Biosyntheseleistung des Stammes an.

1) Man kultivierte den Organismus in unterschiedlichen Fermentationsgefäßen (Schüttelkolben, Rührblattfermenter, Airlifter und P-Kolben). Zusätzlich wurde das Nährmedium variiert und die Salzverträglichkeit von WDMH46 bezüglich $\mathrm{NaCl}$ getestet.

Unter den verschiedenen Fermentationsbedingungen konnten neun weitere Metabolite isoliert und identifiziert werden. Die Aufreinigung erfolgte dabei durch Säulenchromatographie an Kieselgel und Sephadex LH-20 sowie durch präparative HPLC.

Im $5 \mathrm{~L}$ - und $10 \mathrm{~L}$-Rührblattfermenter traten erstmals die Allene $\underline{\mathbf{9 1}}, \underline{\mathbf{9 2}}$ und $\underline{93}$ auf. Neben 4-Hydroxy-3-(3'-hydroxy-3'-methyl-but-1-enyl)-benzoesäure ( $\underline{97})$ wurde ein Metabolit 95 mit Tetrahydrochromanon-Struktur gefunden.

Zwei weitere Allene $(\underline{90 \mathrm{a} / \mathrm{b}})$ wurden vom Stamm beim Zusatz von Natriumacetat zum Nährmedium gebildet. Diese lagen als 3:2-Gemisch vor und wurden aus der Mischung als Isomere identifiziert.

Aus den Rohextrakten der Airlift-Kultur konnten neben Lupallen E ( $\underline{91})$ das $\alpha, \beta$ ungesättigte Keton $\underline{94}$ erhalten werden, ebenso wie $\underline{96}$, eine $p$-Hydroxybenzoe- 
säure mit Dimethylallyl-Seitenkette. Außer $\underline{96}$ sind alle aus diesem Stamm isolierten Naturstoffe neu.<smiles>C=C(C)C=C1C[C@@H](O)C2OC2(CCC(C)CO)C1O</smiles><smiles>C=C(C)C=C=C1C[C@H](O)C2OC2(CCC(C)CO)C1O</smiles><smiles>C=C(C)C=C=C1C[C@H](OC(C)=O)[C@@H](O)[C@](O)(CCC(C)CO)[C@H]1O</smiles><smiles>C=C(C)[14CH2]C=C1CC(OC(C)=O)C2OC2(CCC(C)CO)[C@H]1O</smiles><smiles>C=C(C)/C=C1/C[C@@H](O)[C@H](O)[C@](O)(CCC(C)CO)[C@H]1O</smiles><smiles>C=C(C)C=C=C1C[C@H](O)C(O)[C@]2(CC[C@@H](C)CO2)[C@@H]1O</smiles><smiles>CC(C)=CC(=O)C1CC(O)C2OC2(CCC(C)CO)C1O</smiles><smiles>CC(CO)CCC12OC1C(O)CC1=C2OC(C)(C)CC1=O</smiles><smiles>CC(C)=CCc1cc(C(=O)O)ccc1O</smiles><smiles>CC(C)(O)/C=C\c1cc(C(=O)O)ccc1O</smiles>

2) Aus den Kopplungskonstanten im Protonenspektrum und den NOESY-Korrelationen wurde die relative Stereochemie von $\underline{\mathbf{8 7}}$ und $\underline{\mathbf{9 2}}$ abgeleitet. Zur Klärung der absoluten Konfiguration wurde die Züchtung eines Einkristalls für eine Röntgenstrukturanalyse angestrebt. Kristallisationsversuche mit den Lupallenen A ( $\underline{\mathbf{8}}), \mathrm{C}$ (7) $)$ und $E(\underline{\text { 91) }}$ schlugen jedoch fehl. Um die Kristallisationsfähigkeit der Verbindungen zu erhöhen, wurden $\underline{86}$ zum $p$-Brombenzoat $\underline{100}$ und $\underline{87}$ zum o-Brombenzoat $\underline{99}$ umgesetzt. Bisher blieben auch diese Kristallisationsversuche ohne Erfolg. In Anlehnung an die literaturbekannten Allene $\underline{88}$ und $\underline{\mathbf{8 9}}$ wurde die absolute Konfiguration entsprechend angenommen. 
3) Über die Biosynthese von Allenen ist wenig bekannt. Durch Einbauexperimente mit $\left[1-{ }^{13} \mathrm{C}\right]$ Acetat und $\left[\mathrm{U}-{ }^{13} \mathrm{C}_{3}\right]$ Glycerin konnte aus den ${ }^{13} \mathrm{C}$-NMR-Daten der angereicherten Verbindungen die Bildung der beiden $\mathrm{C}_{5}$-Seitenketten auf dem Mevalonat-Weg bewiesen werden. Für den Cyclohexanring ergab sich ein Aufbau über den Shikimat-Weg mit $p$-Hydroxybenzoesäure als symmetrische Zwischenstufe.

Entsprechend ließ sich die Ausbeute der Lupallene (gemessen an 91) durch den Zusatz von $p$-Hydroxybenzoesäure und Phenylalanin verdoppelt, durch $p$-Hydroxyzimtsäure und Tyrosin sogar vervierfachen.

4) Von den 12 isolierten und aufgeklärten Metaboliten sind einige als Vorläufer auf dem Weg zum Lupallen-Gerüst, andere als Shunt-Produkte einzustufen. Unter Berücksichtigung aller Verbindungen des Stammes WDMH46 wurde ein hypothetisches Biosyntheseschema (Abbildung 64) vorgeschlagen, das erstmals auch über die Allenbildung Auskunft gibt.

5) Für keines der Allene konnte bisher eine biologische Aktivität nachgewiesen werden. 


\section{B. EXPERIMENTELLER TEIL}

\section{Allgemeines}

\section{Instrumentelle Analytik}

\section{Massenspektren:}

EI-MS: Finnigan MAT 95, $70 \mathrm{eV}$;

ESI-MS: Finnigan LC-Q;

HR-ESI-MS: Bruker Apex-Q III, 7 Tesla.

HR-ESI-MS: Bruker Apex IV, FT-ICR 7 Tesla.

DCI-MS: Finnigan MAT 95, 200 eV (Reaktandgas: $\mathrm{NH}_{3}$ );

Hochauflösungen wurden mit Perfluorkerosin als Vergleichssubstanz gemessen, die relativen Intensitäten beziehen sich auf den Basispeak $(I=100 \%)$ und sind in Klammern angegeben.

\section{Infrarotspektren:}

Alle IR-Spektren wurden mit einem FT-IR-Spektrometer der Fa. Perkin-Elmer Modell 1600 als $\mathrm{KBr}$-Preßlinge gemessen.

Abkürzungen: $b r=$ breit, $s h=$ Schulter.

\section{Elektronenspektren (UV):}

Alle Elektronenspektren wurden mit einem Spektrometer der Fa. Varian Modell Cary $3 \mathrm{E}$ gemessen. Methanol/HCl bzw. Methanol/NaOH: $\mathrm{Zu} 2 \mathrm{~mL}$ methanolischer Lösung wurde jeweils ein Tropfen $2 \mathrm{~N} \mathrm{HCl}$ bzw. $2 \mathrm{~N} \mathrm{NaOH}$ gegeben. Die Wellenlänge $\lambda$ ist in [nm] angegeben, der molare Extinktionskoeffizient $\varepsilon$ in $\left[1000 \mathrm{~cm}^{2} \mathrm{~mol}^{-1}\right]$ und wird über nachfolgende Gleichung berechnet (Gleichung gilt für einen Ansatz von $10 \mathrm{~mL}$ Meßlösung):

$$
\varepsilon=\frac{E * \text { Molmasse } * 10}{\text { Einwaage }[\mathrm{mg}]}
$$




\section{Drehwerte:}

Alle Drehwerte wurden mit einem Polarimeter der Fa. Perkin-Elmer Modell 343 bestimmt, die Drehwerte $[\alpha]_{\mathrm{D}}^{20}$ sind in $\left[10^{-1} \mathrm{deg} \mathrm{cm}^{2} / \mathrm{g}\right]$ angegeben, die Konzentrationen $\mathrm{c}$ in $\left[10^{-2} \mathrm{~g} / \mathrm{mL}\right]$, br $=$ breit.

\section{Circulardichroismus-Spektren:}

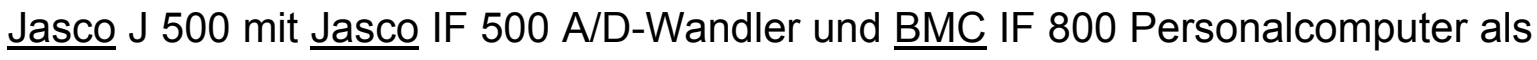
Prozessor. Die molaren Elliptizitäten $\theta$ sind in $\left[10^{-1} \mathrm{grad} \mathrm{cm}^{2} \mathrm{~mol}^{-1}\right]$ angegeben.

\section{H-NMR-Spektren:}

Varian Inova-600 (600 MHz), Varian Inova-500 (500 MHz), Varian Mercury-300 (300 MHz), Varian Unity-300 (300 MHz). Chemische Verschiebungen in $\delta_{\mathrm{H}}-$ Werten (ppm) relativ zum Lösungsmittel als internem Standard; Kopplungskonstanten $(J)$ in Hertz $(\mathrm{Hz})$.

Abkürzungen: $s=$ Singulett, $d=$ Dublett, $t=$ Triplett, $q=$ Quartett, $d d=$ Dublett vom Dublett, $d d d=$ Dublett vom Dublett vom Dublett, $d d d d=$ Dublett vom Dublett vom Dublett vom Dublett, $\mathrm{dt}=$ Dublett vom Triplett, $\mathrm{ddt}=$ Dublett vom Dublett vom Triplett, $\mathrm{td}=$ Triplett vom Dublett, $\mathrm{tdd}=$ Triplett vom Dublett vom Dublett, $\mathrm{tt}=$ Triplett vom Triplett, quin $=$ Quintett, dquin $=$ Dublett vom Quintett, $\mathrm{tm}=$ Triplett im Multiplett, $\mathrm{m}=$ Multiplett, $\mathrm{br}=$ breit.

Alle ${ }^{1} \mathrm{H}-\mathrm{NMR}$-Spektren wurden näherungsweise als Spektren erster Ordnung interpretiert.

\section{${ }^{13}$ C-NMR-Spektren:}

Varian Inova-600 (150.8 MHz), Varian Inova-500 (125.7 MHz), Varian Mercury-

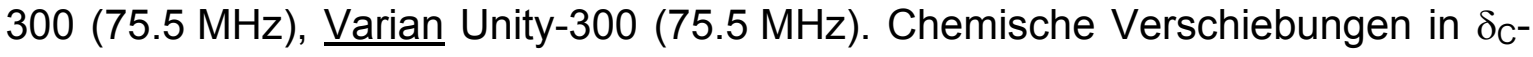
Werten (ppm) relativ zum Lösungsmittel als internem Standard. APT (Attached Proton Test): $\mathrm{CH}$ (d) und $\mathrm{CH}_{3}$ (q) stehen nach oben, $\mathrm{C}(\mathrm{s})$ und $\mathrm{CH}_{2}$ (t) stehen nach unten. ${ }^{13} \mathrm{C}-{ }^{1} \mathrm{H}$-Multiplizitäten sind aus HSQC-Experimenten in Verbindung mit APT-Experimenten ableitbar. Abkürzungen: $s=$ Singulett, $d=$ Dublett, $t=$ Triplett, $q=$ Quartett. 


\section{D-NMR-Spektren:}

${ }^{1} \mathrm{H},{ }^{1} \mathrm{H}$-COSY $\left({ }^{1} \mathrm{H},{ }^{1} \mathrm{H}\right.$-Correlated Spectroscopy), HSQC (Heteronuclear Singular Quantum Coherence), HMBC (Heteronuclear Multiple Bond Connectivity), HMQC (Heteronuclear Multiple Quantum Coherence), NOESY (Nuclear Overhauser Effect Spectroscopy), TOCSY (Total Correlation Spectroscopy).

\section{Chromatographische Methoden}

\section{Dünnschichtchromatographie (DC):}

HPTLC-Nano-Fertigplatten Kieselgel $60 \mathrm{~F}_{254}$ (Merck): $10 \times 10 \mathrm{~cm}$, Schichtdicke $0.2 \mathrm{~mm}$; DC-Alufolien Kieselgel $60 F_{254}$ (Merck): $20 \times 20 \mathrm{~cm}$, Schichtdicke $0.2 \mathrm{~mm}$; DC-Alufolien RP-18 $F_{254 \mathrm{~s}}$ (Merck): $20 \times 20 \mathrm{~cm}$, Schichtdicke: $0.2 \mathrm{~mm}$.

Angegeben sind $\mathrm{R}_{\mathrm{f}}$-Werte (Laufhöhe relativ zur Laufmittelfront).

\section{Säulenchromatographie:}

ICN Kieselgel 60 (KG), 0.032-0.063 mm; Pharmacia Sephadex LH-20; Fluka Sephadex LH-20.

\section{Adsorberharze:}

Serva Amberlite ${ }^{\circledR}$ XAD-2.

\section{Ermittlung von $\mathbf{R}_{\mathbf{f}}$-Werten:}

Zur Bestimmung von $\mathrm{R}_{\mathrm{f}}$-Werten wurden $0.5 \mathrm{mg}$ der $\mathrm{zu}$ untersuchenden Reinsubstanz in einem entsprechenden Lösungsmittel (p.a.) gelöst. Man tüpfelte auf DC-Alufolien, so dass ein Vorlauf von $5 \mathrm{~cm}$ eingehalten wurde. Die Gesamtlaufstrecke des Laufmittels sollte etwa $15 \mathrm{~cm}$ betragen. Die Detektion erfolgte mittels der unten beschriebenen Sprühreagentien.

\section{Sprühreagenzien:}

Nach Merck, Anfärbereagenzien für die Dünnschichtchromatographie. ${ }^{172}$ Die DCPlatten wurden nach dem Ansprühen auf ca. $120^{\circ} \mathrm{C}$ erwärmt.

Anisaldehyd (Anis, Nr. 21): $1 \mathrm{~mL}$ Anisaldehyd gab man in eine Lösung aus $85 \mathrm{~mL}$ Methanol, $10 \mathrm{~mL}$ Eisessig und $5 \mathrm{~mL}$ konz. Schwefelsäure. 
Vanillin-Schwefelsäure (VS, Nr. 322): $1 \mathrm{~g}$ Vanillin wird in $100 \mathrm{~mL}$ konz. Schwefelsäure gegeben.

Orcin-Sprühreagenz (Orcin, Nr. 120-122): $1 \mathrm{~g}$ Eisen-(III)-chlorid wurde in $100 \mathrm{~mL}$ Schwefelsäure gelöst und zu gleichen Anteilen mit einer Orcinlösung $6 \%$ in Ethanol) gemischt.

Ehrlich Reagenz (Ehrlich, Nr. 91): $1 \mathrm{~g}$ 4-Dimethylaminobenzaldehyd wird in einer Mischung von $25 \mathrm{~mL}$ Salzsäure (36 \%) und $75 \mathrm{~mL}$ Methanol gelöst.

\section{High Performance Liquid Chromatography (HPLC):}

\section{- LC-MS:}

Pumpe: Flux Instruments Rheos 4000; Autosampler: Jasco AS-851 (0 - $100 \mu \mathrm{L}$ variable Aufgabeschleife); UV-Detektor: Linear UVIS-205; Massendetektor: Finnigan LC-Q; Entgaser: Flux Instruments ERC-3415 $\alpha$; Steuersoftware HPLC: Flux Instruments Janeiro; Datensystem: Finnigan Xcalibur; Vorsäule: Grom Supersphere 100 RP-18 endcapped, 4 mm, $10 \times 2$ mm; Säule: Grom Supersphere 100 RP-18 endcapped, $4 \mu \mathrm{m}, 100 \times 2 \mathrm{~mm}$.

Programm: von 20\% Methanol auf 100\% Methanol in $20 \mathrm{~min}, 5 \mathrm{~min} 100 \%$ Methanol, Flussrate: $300 \mu \mathrm{L} / \mathrm{min}$; UV-Detektion: $254 \mathrm{~nm}$, Masse: positive und negative lonen.

\section{- HPLC 1 (analytisch):}

Pumpe: Kontron P 322 System; Autosampler: Kontron Sa 360; Detektor: Kontron Dioden Array Detektor 440; Mischkammer: Kontron HPLC 360; Software: Kontron Kroma System 2000 Version 1.60; analytische Aufgabenschleife: $20 \mu \mathrm{L}$;

Säulen:

1: Jasco Kromasil $100 \mathrm{C} 18,5 \mu \mathrm{m}, 4 \mathrm{~mm} \times 250 \mathrm{~mm}$.

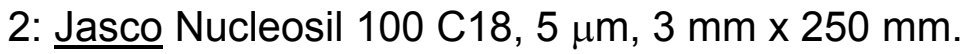

Programme:

1: isokratisch $23 \% \mathrm{AcCN}$ mit $0.1 \%$ TFA und $77 \%$ Wasser mit $0.1 \mathrm{TFA}$, Flussrate: $0.6 \mathrm{~mL} / \mathrm{min}$, UV-Detektion bei $254 \mathrm{~nm}$.

2: isokratisch $30 \%$ AcCN mit $0.1 \%$ TFA und $70 \%$ Wasser mit $0.1 \%$ TFA, Flussrate: $0.5 \mathrm{~mL} / \mathrm{min}$, UV-Detektion bei $225 \mathrm{~nm}$.

3: Lösung A: Wasser mit 0.1\%ige TFA und Lösung $B$ : AcCN mit 0.1\% TFA, Gradient von 80\% Lösung A zu 40\% Lösung B in 20 min, in 1 min auf 100\% 
Lösung B, von $100 \%$ Lösung B zu $80 \%$ Lösung $A$ in 2 min, Flußrate 0.5 $\mathrm{mL} / \mathrm{min}$, Detektion bei 225 und $254 \mathrm{~nm}$.

\section{- HPLC 2 (analytisch):}

Pumpe: Jasco PU-1580; Detektoren: Jasco UV-1570 M, CD-995; Software: Jasco Borwin Version 1.50; Mischeinheit: Jasco LG-1580-02; Lösungsmittelentgaser: Jasco DG-1580-53; Autosampler: Jasco AS 1555;

Säule:

3: Jasco Kromasil $100 \mathrm{C} 18,5 \mu \mathrm{m}, 250 \times 8 \mathrm{~mm}$. Programme:

4: Lösung A: Wasser mit $0.1 \%$ ige TFA und Lösung B: AcCN mit 0.1\% TFA, Gradient von $70 \%$ Lösung A zu 90\% Lösung B in 20 min, 10 min bei $90 \%$ Lösung $B$, von $90 \%$ Lösung $B$ zu $70 \%$ Lösung $A$ in 2 min, Flußrate $0.8 \mathrm{~mL} / \mathrm{min}$, Detektion bei 225 und $254 \mathrm{~nm}$.

1: isokratisch: $23 \% \mathrm{AcCN}$ mit $0.1 \%$ TFA und $77 \%$ Wasser mit 0.1 TFA, Flussrate: $0.6 \mathrm{~mL} / \mathrm{min}$, UV-Detektion bei $254 \mathrm{~nm}$.

\section{- HPLC 3 (präparativ):}

Pumpe: Jasco PU-1587; UV-Detektor: Jasco UV/VIS 1575; Software: Jasco Borwin Version 1.50; präparative Aufgabenschleife: $2 \mathrm{~mL}$;

Säulen:

4: Jasco Kromasil $100 \mathrm{C} 18,5 \mu \mathrm{m}, 8 \mathrm{~mm} \times 250 \mathrm{~mm}$.

5: Jasco Nucleosil $100 \mathrm{C} 18,5 \mu \mathrm{m}, 8 \mathrm{~mm} \times 250 \mathrm{~mm}$.

Programme:

5: isokratisch: $25 \% \mathrm{AcCN}$ und $75 \%$ Wasser (säurefrei), Flussrate: $2.5 \mathrm{~mL} / \mathrm{min}$, UV-Detektion bei $254 \mathrm{~nm}$.

6: isokratisch: $28 \% \mathrm{AcCN}$ und $72 \% \mathrm{H}_{2} \mathrm{O}$ (säurefrei); Flussrate: $2.5 \mathrm{~mL} / \mathrm{min}$; UVDetektion bei $225 \mathrm{~nm}$.

7: isokratisch: $30 \%$ AcCN und $70 \%$ Wasser (säurefrei), Flussrate: $2.5 \mathrm{~mL} / \mathrm{min}$, UV-Detektion bei $225 \mathrm{~nm}$.

\section{Lösungsmittel:}

Lösungsmittel für die Säulenchromatographie wurden zuvor destilliert. Für die HPLC wurden nur analysenreine Lösungsmittel (LiChrosolv, Merck) und bidestilliertes Wasser verwendet. Vor Benutzung entgaste man Lösungsmittel für die 
HPLC durch jeweils 15 Minuten langes Behandeln im Ultraschallbad und anschließendes Durchleiten von Helium.

\section{Mikrobiologische Methoden}

Alle mikrobiologischen Arbeiten wurden unter den üblichen sterilen Bedingungen durchgeführt. ${ }^{173}$

\section{Nährmedienbestandteile:}

Die verwendeten Nährmedienbestandteile wurden von folgenden Firmen bezogen: Merck: Malzextrakt, D-Glucose, Fleischextrakt, Caseinpepton.

Henselwerk $\mathrm{GmbH}$ : entfettetes Sojamehl

Gibco BRL: Hefeextrakt

Oxoid: Hefeextrakt

Neuform: Hafermehl (Holo Hafergold)

Difco: BiTek Agar-Agar

AppliChem: D-Glucose

Fluka: Maltose

Villa Natura: Biomalz

\section{Nährmedien:}

Alle Nährmedien wurden 30 min bei $121^{\circ} \mathrm{C}$ und einem bar Überdruck sterilisiert. Mengenangaben beziehen sich jeweils auf einen Liter demineralisiertes Wasser, der $\mathrm{pH}$-Wert wurde vor der Sterilisation mit $0.5 \mathrm{M} \mathrm{NaOH}$ bzw. $0.5 \mathrm{M} \mathrm{HCl}$ eingestellt. Festen Medien wurde noch $22 \mathrm{~g} / \mathrm{L}$ Agar hinzugefügt.

NL 1158:

$20 \mathrm{~g}$ Malzextrakt

$10 \mathrm{~g}$ Glucose

$2 \mathrm{~g} \mathrm{Hefeextrakt}$

$0.5 \mathrm{~g}$ Ammoniumhydrogenphosphat $\mathrm{pH}=6.5$.
NL 1549:

$10 \mathrm{~g}$ Glucose

$10 \mathrm{~g}$ Maltose

$5 \mathrm{~g}$ Caseinpepton

$5 \mathrm{~g}$ Fleischpepton

$\mathrm{pH}=5.4$. 
NL E2:

$20 \mathrm{~g}$ Glucose

$20 \mathrm{~g}$ Hafermehl

$20 \mathrm{~g}$ entfettetes Sojamehl

keine $\mathrm{pH}$-Einstellung.

NL 1405:

$30 \mathrm{~g}$ Glucose

$3 \mathrm{~g}$ Natriumnitrat

$1 \mathrm{~g}$ Kaliumhydrogenphosphat

$0.5 \mathrm{~g}$ Magnesiumsulfat

$0.5 \mathrm{~g}$ Kaliumchlorid

$0.01 \mathrm{~g}$ Eisensulfat

$\mathrm{pH}=7.3$.
NL G20:

$20 \mathrm{~g}$ Glycerin

$10 \mathrm{~g}$ Malzextrakt

$4 \mathrm{~g}$ Hefeextrakt

keine $\mathrm{pH}$-Einstellung.

NL MB:

$37.4 \mathrm{~g}$ Marine Broth

$\mathrm{pH}=7.0$.

NL Biomalz:

$20 \mathrm{~g}$ Biomalz

$\mathrm{pH}=5.6$.

\section{Schüttler und Fermenter:}

Braun Certomat BS-1, Braun Certomat HK, Schüttelbrett Universität Göttingen, Braun Biostat M (1 L), Braun Biostat B (2 L, $5 \mathrm{~L})$, Braun Biostat E (10 L), $10 \mathrm{~L}-$ Airlift-Fermenter, gebaut: Uni Dortmund (10 L, 4 vvm), Fischer \& Porter Gasflowmeter (45711M), The Analytical Development Co Ltd. $\mathrm{CO}_{2}$-Analysator, Ingold $\mathrm{O}_{2}$ Elektrode.

\section{Plattendiffusionstest:}

Auf die mit verschiedenen Testkeimen angeimpften Agarplatten wurden Filterplättchen (Durchmesser: $6 \mathrm{~mm}$, Dicke: $0.5 \mathrm{~mm}$ ) gelegt, die mit $15 \mu \mathrm{L}$ Substanzlösung (in Methanol oder Chloroform gelöst, $c=1 \mathrm{mg} / \mathrm{mL}$ ) getränkt und vor dem Auflegen unter sterilen Bedingungen getrocknet worden waren. Als Testkeime dienten Bacillus subtilis, Escherichia coli, Staphylococcus aureus und Candida albicans. Die Agarplatten wurden $24 \mathrm{~h}$ bei $25^{\circ} \mathrm{C}$ (C. albicans) bzw. $37^{\circ} \mathrm{C}(B$. subtilis, E. coli und S. aureus) inkubiert. 
Zusammensetzung der Nährböden für die Plattendiffusionstests:

B. subtilis und E. coli: $5 \mathrm{~g} / \mathrm{L}$ Glucose, $0.5 \mathrm{~g} / \mathrm{L}$ Trinatriumcitrat $\times 2 \mathrm{H}_{2} \mathrm{O}, 3 \mathrm{~g} / \mathrm{L}$ $\mathrm{KH}_{2} \mathrm{PO}_{4}, 7 \mathrm{~g} / \mathrm{L} \mathrm{K}_{2} \mathrm{HPO}_{4}, 0.1 \mathrm{~g} / \mathrm{L} \mathrm{MgSO}_{4} \times 7 \mathrm{H}_{2} \mathrm{O}, 1 \mathrm{~g} / \mathrm{L}\left(\mathrm{NH}_{4}\right)_{2} \mathrm{SO}_{4}$ und $15 \mathrm{~g} / \mathrm{L}$ Agar, keine $\mathrm{pH}$-Einstellung.

S. aureus: $8 \mathrm{~g} / \mathrm{L}$ Bacto Nutrient Broth, $5 \mathrm{~g} / \mathrm{L} \mathrm{NaCl}$ und $15 \mathrm{~g} / \mathrm{L}$ Agar, keine $\mathrm{pH}$-Einstellung.

C. albicans: $4 \mathrm{~g} / \mathrm{L}$ Hefeextrakt, $10 \mathrm{~g} / \mathrm{L}$ Malzextrakt, $4 \mathrm{~g} / \mathrm{L}$ Glucose und $15 \mathrm{~g} / \mathrm{L}$ Agar, $\mathrm{pH}=5.5$.

Zum Ansetzen der Testplatten-Medien wurde demineralisiertes Wasser verwendet. Die Glucose wurde erst nach der 30 minütigen Sterilisation hinzugegeben.

\section{UV-Lampe:}

$\underline{\text { Hanau }}$ Floutest, Benda Labor- und UV-Strahler.

\section{Ultraschallbad:}

Bandelin Sonorex RK 106 S.

\section{Arbeiten an marinen Pilzstämmen}

\section{Stammhaltung}

\subsection{Agarplatten}

Die Haltung der marinen Pilzstämme erfolgte auf Agarplatten mit den Nährmedien 1158, E2 und G20. Die Agarplatten wurden mit dem jeweiligen Stamm beimpft, 7 Tage bei $25^{\circ} \mathrm{C}$ inkubiert und anschließend bei $4{ }^{\circ} \mathrm{C}$ gelagert. Zum Beimpfen der Flüssigkulturen wurden keine Platten verwendet, die älter als 3 Wochen waren.

\subsection{Stickstoff-Einlagerungen}

Aus einer gut bewachsenen Agarplatte wurden mit einseitig zugeschmolzenen und mit einem Bleistück beschwerten sterilen Halmen aus Polypropylen Agarstückchen ausgestanzt, bis der Halm gefüllt war. Dieser wurde in einen zweiten 
äußeren Halm gesteckt, der mit einer ausgeglühten Tiegelzange an beiden Seiten zugeschmolzen wurde. Die Halme wurden in einem Dewar über flüssigem Stickstoff bei $-196{ }^{\circ} \mathrm{C}$ gelagert.

\subsection{Glycerin-Einlagerungen}

Für die Glycerin-Einlagerungen wurde eine vier Tage alte Vorkultur hergestellt. Dazu wurde ein $1 \mathrm{~L}$ Erlenmeyerkolben ohne Schikanen mit $170 \mathrm{~mL}$ Nährlösung beschickt, mit einem $3 \mathrm{~cm}^{2}$ großem Stück einer gut bewachsenen Agarplatte beimpft und bei $28{ }^{\circ} \mathrm{C}$ und $120 \mathrm{spm}$ inkubiert. Von dieser Kultur nahm man jeweils $2 \mathrm{~mL} a b$, versetzte sie mit $4 \mathrm{~mL}$ sterilem Glycerin und vermischte gut. Die Mischung wurde anschließend im Gefrierfach gelagert.

\section{Durchführung des chemischen Screenings}

Die Kultivierung der zu untersuchenden Stämme erfolgte in sechs verschiedenen Medien (1158, E2, G20, 1405, 1549 und Marine Broth) in $1 \mathrm{~L}$ Erlenmeyerkolben ohne Schikanen, die jeweils mit $170 \mathrm{~mL}$ Nährlösung gefüllt waren. Die Kolben wurden jeweils mit einem $3 \mathrm{~cm}^{2}$ großem Stück einer gut bewachsenen Agarplatte beimpft und bei $28^{\circ} \mathrm{C}$ und $120 \mathrm{spm}$ inkubiert. Nach 4 bzw. 6 Tagen wurden jeweils zwei Kolben geerntet. In die vereinigten Kulturbrühen $(340 \mathrm{~mL})$ wurde Celite gegeben und zentrifugiert. Das Myzel wurde $15 \mathrm{~min}$ im Ultraschallbad mit $200 \mathrm{~mL}$ Aceton/Methanol 1:1 extrahiert, durch Filtration abgetrennt und das Lösungsmittel im Vakuum entfernt. Das Kulturfiltrat wurde dreimal mit $200 \mathrm{~mL}$ Essigester extrahiert und die organische Phase eingeengt. Die so erhaltenen Extrakte (Kulturfiltrat und Myzel) wurden in $2 \mathrm{~mL} \mathrm{MeOH} / \mathrm{H}_{2} \mathrm{O}$ 5:1 gelöst und $5 \mu \mathrm{L}$ dieser Lösung auf eine HPTLC-Kieselgelplatte aufgetragen. Die Dünnschichtchromatogramme wurden in $\mathrm{CHCl}_{3} / \mathrm{MeOH}$ 9:1 und $n-\mathrm{BuOH} /$ Eisessig/ $\mathrm{H}_{2} \mathrm{O}$ 5:1:4 entwickelt. Die Analyse des Metabolitenmusters erfolgte durch UV-Licht (254 und $366 \mathrm{~nm}$ ), Eigenfarbe und mit Hilfe von Sprühreagenzien (Anis, Orcin, VS und Ehrlich). 


\section{Stamm WDMH 35}

\subsection{Kultivierung und Aufarbeitung}

Der Stamm WDMH 35 (Aspergillus sp.) wurde in einem $5 \mathrm{~L}$-Ansatz in $1 \mathrm{~L}$ Erlenmeyerkolben ohne Schikanen, die jeweils mit $170 \mathrm{~mL}$ Nährlösung G20 gefüllt waren, kultiviert. Die Kolben wurden jeweils mit einem $3 \mathrm{~cm}^{2}$ großem Stück einer gut bewachsenen Agarplatte beimpft und bei $28^{\circ} \mathrm{C}$ und $120 \mathrm{spm}$ inkubiert. Nach sechs Tagen wurden die Kolben geerntet. In die vereinigte Kulturbrühe wurde Celite gegeben und zentrifugiert. Das Myzel wurde $15 \mathrm{~min}$ im Ultraschallbad mit $1 \mathrm{~L}$ Aceton/Methanol 1:1 extrahiert, durch Filtration abgetrennt und das Lösungsmittel im Vakuum entfernt. Das Kulturfiltrat wurde dreimal mit $1 \mathrm{~L}$ Essigester extrahiert und die organische Phase eingeengt.

\subsection{Isolierung}

Der Kulturfiltratextrakt wurde an Kieselgel $\left(\mathrm{CH}_{2} \mathrm{Cl}_{2} / \mathrm{MeOH} 20: 1 \rightarrow 7: 1\right)$ säulenchromatographisch getrennt. Anschließende Aufreinigung der Fraktion 5 an Sephadex LH-20 (Aceton) und Kieselgel (EE/Cyclohexan 4:1) lieferten $51.3 \mathrm{mg}$ cis-Pseurotin (29). Die Aufreinigung der 6. Fraktion über Kieselgel (EE/Methanol 19:1) und Sephadex LH-20 (Methanol) ergaben 7.7 mg Fumigaclavin A (1ㅜ).

Der Myzelextrakt wurde an Kieselgel $\left(\mathrm{CH}_{2} \mathrm{Cl}_{2} / \mathrm{MeOH} 20: 1 \rightarrow 9: 1\right)$ und (EE/ Methanol 19:1) chromatographiert. Die erhaltene Fraktion 4 wurde mit einer vorgereinigten Kulturfiltratfraktion 2 [Kieselgel $\left(\mathrm{CH}_{2} \mathrm{Cl}_{2} / \mathrm{MeOH}\right.$ 7:1), (EE/Cyclohexan 4:1) und Sephadex LH-20 (Methanol)] vereinigt und über eine Sephadex $\mathrm{LH}-20\left(\mathrm{CH}_{2} \mathrm{Cl}_{2} / \mathrm{MeOH}\right.$ 1:1) aufgereinigt. Man erhielt $32.4 \mathrm{mg}$ Fumitremorgin $\mathrm{C}(\underline{\mathbf{3 2}})$. Das Aufarbeitungsschema ist im Theoretischen Teil dieser Arbeit dargestellt (Abbildung 5).

\subsection{Charakterisierung der Metabolite}

cis-Pseurotin (모)

$\mathrm{C}_{22} \mathrm{H}_{25} \mathrm{NO}_{8}(431.44)$<smiles>C[13CH2]/C=C\[C@@H](O)[C@@H](O)C1=C(C)C(=O)[C@]2(O1)C(=O)N[C@](OC)(C(=O)c1ccccc1)[C@@H]2O</smiles> 
$\mathbf{R}_{\mathbf{f}}$-Wert: 0.44 (Chloroform/Methanol 9:1)

0.47 (Cyclohexan/Essigester/Methanol 5:10:2)

0.54 (Essigester/Methanol 19:1).

Anfärbeverhalten: ockergelb (Anis), grünlich (Orcin), hellbraun (Ehrlich).

$[\alpha]_{D}^{20}=-4^{\circ}\left(\mathrm{c}=1.0 \mathrm{mg} / \mathrm{mL}\right.$ in Methanol), $\left(\mathrm{Litt}^{48}[\alpha]_{D}^{20}=-5^{\circ}(\mathrm{MeOH})\right)$.

ESI-MS: $\mathrm{m} / \mathrm{z}=885.3[2 \mathrm{M}+\mathrm{Na}]^{+}, 454.6[\mathrm{M}+\mathrm{Na}]^{+}, 430[\mathrm{M}-\mathrm{H}]^{-}$.

IR $(\mathrm{KBr}): \tilde{v}=3475,3386,3309,2963,1722,1703,1630,1392,1351,1174,1093$, $1081,1050,1022,687,668 \mathrm{~cm}^{-1}$.

UV $(\mathrm{MeOH}) \quad: \lambda_{\max }(\varepsilon)=203$ (15157), 253 (10680), 279 (7459);

$(\mathrm{MeOH} / \mathrm{HCl}) \quad: \lambda_{\max }(\varepsilon)=202$ (16530), 253 (10354), 279 (7238);

$(\mathrm{MeOH} / \mathrm{NaOH}): \lambda_{\max }(\varepsilon)=205$ (15417), 251 (10802), 280 (6973) nm.

CD $(\mathrm{MeOH}): \lambda_{\max }[\Theta]=202$ (58131), 233 (-10634), 251 (15313), 277 (39528), 313 (10788), $350(-4283) \mathrm{nm}$.

${ }^{1} \mathrm{H}-\mathrm{NMR}\left(500 \mathrm{MHz}, \mathrm{CD}_{3} \mathrm{OD}\right): \delta_{\mathrm{H}}=0.97\left(\mathrm{t}, J=7.5 \mathrm{~Hz}, 3 \mathrm{H}, 15-\mathrm{H}_{3}\right), 1.75(\mathrm{~s}, 3 \mathrm{H}$, 16- $\left.\mathrm{H}_{3}\right), 2.13\left(\mathrm{~m}, 2 \mathrm{H}, 14-\mathrm{H}_{2}\right), 3.34\left(\mathrm{~s}, 3 \mathrm{H}, 8-\mathrm{OCH}_{3}\right), 4.50$ (d, J = 7.0 Hz, 1H, 10-H), $4.54(\mathrm{~s}, 1 \mathrm{H}, 9-\mathrm{H}$ ), 4.67 (dd, $J=8.5,6.5 \mathrm{~Hz}, 1 \mathrm{H}, 11-\mathrm{H}$ ), 5.46 (ddt, $J=10.5,9.0$, $1.5 \mathrm{~Hz}, 1 \mathrm{H}, 12-\mathrm{H}$ ), 5.61 (dt, $J=11.0,7.5 \mathrm{~Hz}, 1 \mathrm{H}, 13-\mathrm{H}), 7.49(\mathrm{t}, J=8.0 \mathrm{~Hz}, 2 \mathrm{H}$, $20-\mathrm{H}, 22-\mathrm{H}), 7.63(\mathrm{t}, J=7.5 \mathrm{~Hz}, 1 \mathrm{H}, 21-\mathrm{H}), 8.35(\mathrm{~m}, 2 \mathrm{H}, 21-\mathrm{H}, 23-\mathrm{H}) \mathrm{ppm}$.

${ }^{13}$ C-NMR (125.7 MHz, CD ${ }_{3} \mathrm{OD}$ ): $\delta_{\mathrm{C}}=5.84$ (q, C-16), 14.54 (q, C-15), 22.22 (t, C-14), 52.47 (q, 8- $\mathrm{OCH}_{3}$ ), 69.55 (d, C-11), 72.90 (d, C-10), 76.28 (d, C-9), 93.55 (s, C-8), 93.81 (s, C-5), 114.41 (s, C-3), 128.73 (d, C-12), 129.52 (d, C-20, C-22), 131.67 (d, C-19, C-23), 134.82 (s, C-18), 135.14 (d, C-21), 137.30 (d, C-13), 169.14 (s, C-2), 188.66 (s, C-6), 197.07 (s, C-17),199.19 (s, C-4) ppm. 
Fumigaclavin A ( $\underline{31})$

$\mathrm{C}_{18} \mathrm{H}_{22} \mathrm{~N}_{2} \mathrm{O}_{2}(298.38)$

$\mathbf{R}_{\mathbf{f}}$-Wert: 0.38 (Chloroform/Methanol 9:1)

0.27 (Cyclohexan/Essigester/Methanol 5:10:2)

0.39 (Essigester/Methanol 19:1).

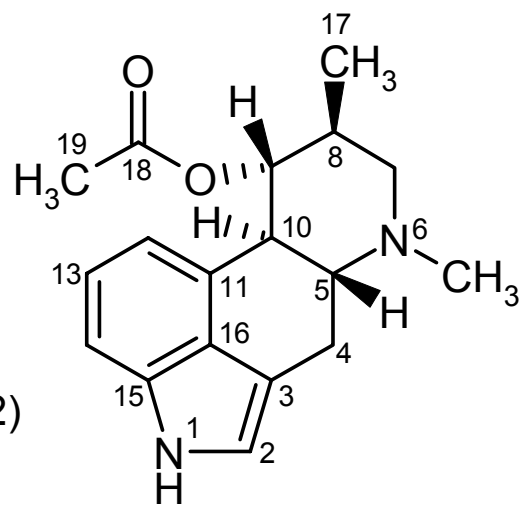

Anfärbeverhalten: pink (Anis), lila-grau (Orcin), lila (Ehrlich).

$[\alpha]_{D}^{20}=-46^{\circ}\left(\mathrm{c}=1.0 \mathrm{mg} / \mathrm{mL}\right.$ in Methanol), $\left(\mathrm{Lit}^{60}[\alpha]^{i}=-54^{\circ}\left(\mathrm{CHCl}_{3}\right)\right)$.

El-MS: $\mathrm{m} / \mathrm{z}(\%)=298(55)[\mathrm{M}]^{+}, 239(100)$.

IR $(\mathrm{KBr}): \tilde{v}=3404,2961,2852,1724,1624,1447,1377,1351,1248,1083,1024$ $752 \mathrm{~cm}^{-1}$.

UV (MeOH) $\quad: \lambda_{\max }(\varepsilon)=203$ (15039), 224 (15829), 258 (5792), 280 (5987);

$(\mathrm{MeOH} / \mathrm{HCl}) \quad: \lambda_{\max }(\varepsilon)=202$ (15822), 222 (16255), 257 (5759), 280 (6030);

$(\mathrm{MeOH} / \mathrm{NaOH}): \lambda_{\max }(\varepsilon)=206$ (14749), 224 (17262), 254 (6200), 280 (6151) nm.

CD $(\mathrm{MeOH}): \lambda_{\max }[\Theta]=206$ (8176), 210 (6721), 213 (7816), 217 (4541), 228 (8404), 237 (811), 248 (6013), 278 (-18194), 313 (3438), 351 (-2097) nm.

${ }^{1} \mathrm{H}-\mathrm{NMR}\left(500 \mathrm{MHz}, \mathrm{CD}_{3} \mathrm{OD}\right): \delta_{\mathrm{H}}=1.31\left(\mathrm{dd}, J=7.0,1.5 \mathrm{~Hz}, 3 \mathrm{H}, 17-\mathrm{H}_{3}\right), 1.82(\mathrm{~s}$, $\left.3 \mathrm{H}, 19-\mathrm{H}_{3}\right), 2.06(\mathrm{~m}, 1 \mathrm{H}, 8-\mathrm{H}), 2.42\left(\mathrm{~d}, J=2.0 \mathrm{~Hz}, 3 \mathrm{H}, 6-\mathrm{NCH}_{3}\right), 2.61(\mathrm{~m}, 1 \mathrm{H}, 5-\mathrm{H})$, $2.62\left(\mathrm{~m}, 1 \mathrm{H}, 4-\mathrm{H}_{\mathrm{A}}\right), 2.69\left(\mathrm{~m}, 2 \mathrm{H}, 7-\mathrm{H}_{2}\right), 3.30(\mathrm{~m}, 1 \mathrm{H}, 10-\mathrm{H}), 3.42\left(\mathrm{~m}, 1 \mathrm{H}, 4-\mathrm{H}_{\mathrm{B}}\right)$, 5.63 (quin, $J=1.5 \mathrm{~Hz}, 1 \mathrm{H}, 9-\mathrm{H}$ ), 6.62 (dd, $J=8.0,0.5 \mathrm{~Hz}, 1 \mathrm{H}, 12-\mathrm{H}), 6.89(\mathrm{~s}, 1 \mathrm{H}$, 2-H), $7.00(\mathrm{t}, J=8.0 \mathrm{~Hz}, 1 \mathrm{H}, 13-\mathrm{H}), 7.10(\mathrm{dd}, J=8.0,0.5 \mathrm{~Hz}, 1 \mathrm{H}, 14-\mathrm{H}) \mathrm{ppm}$.

${ }^{13}$ C-NMR (125.7 MHz, CD ${ }_{3} \mathrm{OD}$ ): $\delta_{\mathrm{C}}=16.94$ (q, C-17), 20.97 (q, C-19), 27.30 (t, C-4), 34.47 (d, C-8), 40.79 (d, C-10), 43.94 (q, 6- $\mathrm{NCH}_{3}$ ), 58.90 (t, C-7), 63.48 (d, C-5), 72.88 (d, C-9), 109.63 (d, C-14), 110.96 (s, C-3), 112.91 (d, C-12), 119.36 (d, C-2), 123.33 (d, C-13), 128.07 (s, C-16), 130.08 (s, C-11), 135.37 (s, C-15), 172.57 (s, C-18) ppm. 
Fumitremorgin C (르)

$\mathrm{C}_{22} \mathrm{H}_{25} \mathrm{~N}_{3} \mathrm{O}_{3}(379.46)$

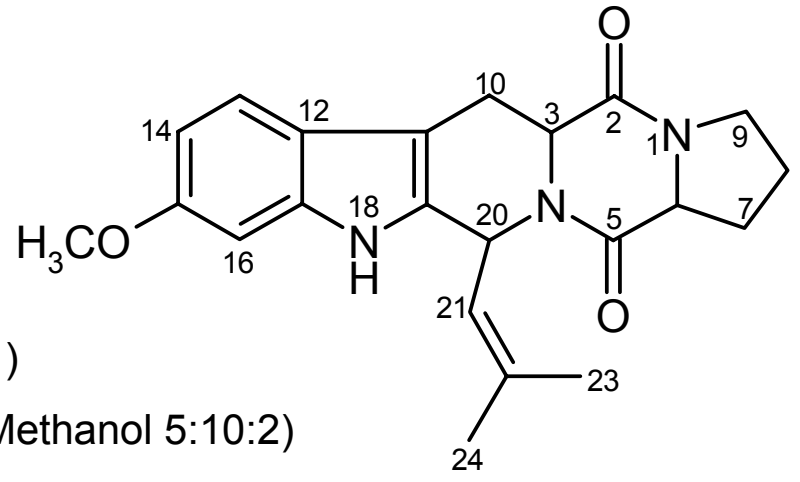

$\mathbf{R}_{\mathbf{f}}$-Wert: 0.62 (Chloroform/Methanol 9:1)

0.40 (Cyclohexan/Essigester/Methanol 5:10:2)

0.39 (Essigester/Methanol 19:1).

Anfärbeverhalten: grün (Anis), lila-blau (Orcin), orange-braun (Ehrlich).

$[\alpha]_{D}^{20}=-19^{\circ}(\mathrm{c}=1.0 \mathrm{mg} / \mathrm{mL}$ in Methanol $)$.

El-MS: $\mathrm{m} / \mathrm{z}(\%)=379.3(100)[\mathrm{M}]^{+}, 281.2(50)$.

IR $(\mathrm{KBr}): \tilde{v}=3532,3445,3300,2926,2853,1660,1471,1414,1328,1296,1280$, $1254,1227,1200,1156,1034,920,821 \mathrm{~cm}^{-1}$.

UV (MeOH) : $\quad \lambda_{\max }(\varepsilon)=206$ (26371), 223 (29777), 272 (5909), 297 (6762);

$(\mathrm{MeOH} / \mathrm{HCl}) \quad: \lambda_{\max }(\varepsilon)=205$ (29448), 222 (31269), 271 (6623), 298 (7289);

$(\mathrm{MeOH} / \mathrm{NaOH}): \lambda_{\max }(\varepsilon)=207$ (26086), 223 (31625), 271 (6789), 297 (7558) nm.

CD (MeOH): $\lambda_{\max }[\Theta]=203$ (894), 206 (-41405), 216 (-8360), 226 (-25119), 240 (762), 246 (-430), 272 (21078), 284 (8051), 298 (13086) nm.

${ }^{1} \mathrm{H}-\mathrm{NMR}\left(500 \mathrm{MHz}, \mathrm{CDCl}_{3}\right): \delta_{\mathrm{H}}=1.62\left(\mathrm{~s}, 3 \mathrm{H}, 23-\mathrm{H}_{3}\right), 1.90\left(\mathrm{~m}, 1 \mathrm{H}, 8-\mathrm{H}_{\mathrm{A}}\right), 1.96(\mathrm{brs}$, $\left.3 \mathrm{H}, 24-\mathrm{H}_{3}\right), 2.02\left(\mathrm{~m}, 1 \mathrm{H}, 8-\mathrm{H}_{\mathrm{B}}\right), 2.20\left(\mathrm{~m}, 1 \mathrm{H}, 7-\mathrm{H}_{\mathrm{A}}\right), 2.37\left(\mathrm{~m}, 1 \mathrm{H}, 7-\mathrm{H}_{\mathrm{B}}\right), 3.07(\mathrm{dd}$, $\left.J=16.0,11.5 \mathrm{~Hz}, 1 \mathrm{H}, 10-\mathrm{H}_{\mathrm{A}}\right), 3.49\left(\mathrm{dd}, J=16.0,5.0 \mathrm{~Hz}, 1 \mathrm{H}, 10-\mathrm{H}_{\mathrm{B}}\right), 3.61(\mathrm{~m}, 2 \mathrm{H}$, 9- $\mathrm{H}_{2}$ ), 3.80 (d, $\left.J=1.5 \mathrm{~Hz}, 3 \mathrm{H}, 15-\mathrm{OCH}_{3}\right), 4.07$ (brt, $\left.J=7.5 \mathrm{~Hz}, 1 \mathrm{H}, 6-\mathrm{H}\right), 4.17$ (brdd, $J=12.0,4.5 \mathrm{~Hz}, 1 \mathrm{H}, 3-\mathrm{H}$ ), 4.88 (dquin, $J=9.5,1.0 \mathrm{~Hz}, 1 \mathrm{H}, 21-\mathrm{H}$ ), 5.97 (d, $J=9.5 \mathrm{~Hz}, 1 \mathrm{H}, 20-\mathrm{H}), 6.78(\mathrm{dd}, J=8.5,1.5 \mathrm{~Hz}, 1 \mathrm{H}, 14-\mathrm{H}), 6.82(\mathrm{t}, J=1.5 \mathrm{~Hz}, 1 \mathrm{H}$, $16-\mathrm{H}), 7.40$ (dd, $J=8.5,1.0 \mathrm{~Hz}, 1 \mathrm{H}, 13-\mathrm{H}), 7.64(\mathrm{~m}, 1 \mathrm{H}, 18-\mathrm{NH}) \mathrm{ppm}$.

${ }^{13}$ C-NMR (125.7 MHz, CDCl 3 ): $\delta_{\mathrm{C}}=17.99$ (q, C-24), 21.85 (t, C-10), 22.96 (t, C-8), 25.63 (q, C-23), 28.48 (t, C-7), 45.33 (t, C-9), 50.93 (d, C-20), 55.67 (q, 15- $\mathrm{OCH}_{3}$ ), 56.70 (d, C-3), 59.13 (d, C-6), 95.26 (d, C-16), 105.99 (s, C-11), 109.28 (d, C-14), 
118.75 (d, C-13), 120.66 (s, C-12), 124.05 (d, C-21), 132.18 (s, C-19), 133.92 (s, C-22), 136.98 (s, C-17), 156.34 (s, C-15), 165.72 (s, C-2), 169.44 (s, C-5) ppm.

\section{Stamm WDMH C2}

\subsection{Kultivierung und Aufarbeitung}

Ein 5 L-Ansatz wurde in $1 \mathrm{~L}$-Erlenmeyerkolben ohne Schikanen, die jeweils mit 170 mL Nährlösung 1158 gefüllt waren, durchgeführt. Dazu wurden die Kolben jeweils mit einem $3 \mathrm{~cm}^{2}$ großem Stück einer gut bewachsenen Agarplatte des Stamm WDMH C2 beimpft und bei $28^{\circ} \mathrm{C}$ und $120 \mathrm{spm}$ inkubiert. Nach sechs Tagen wurden die Kolben geerntet. Die Aufarbeitung erfolgte nach Abbildung 4. In die vereinigten Kulturbrühen wurde Celite gegeben und zentrifugiert. Das Myzel wurde $15 \mathrm{~min}$ im Ultraschallbad mit $1 \mathrm{~L}$ Aceton/Methanol 1:1 extrahiert, durch Filtration abgetrennt und das Lösungsmittel im Vakuum entfernt. Das Kulturfiltrat wurde dreimal mit $1 \mathrm{~L}$ Essigester extrahiert und die organische Phase eingeengt.

\subsection{Cyclonerodiol (느)}

Der Kulturfiltratextrakt wurde an Kieselgel $\left(\mathrm{CH}_{2} \mathrm{Cl}_{2} / \mathrm{MeOH}\right.$ 99:1 $\left.\rightarrow 32: 1\right)$ chromatographiert. Eine weitere KG-Säule (EE/Cyclohexan 2:1) lieferten $6.1 \mathrm{mg}$

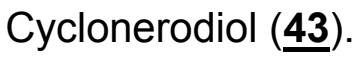

$\mathrm{C}_{15} \mathrm{H}_{28} \mathrm{O}_{2}(240.39)$

$\mathbf{R}_{\mathbf{f}}$-Wert: 0.46 (Chloroform/Methanol 9:1)

0.54 (Cyclohexan/Essigester/Methanol 5:10:2)

0.54 (Essigester/Methanol 19:1).

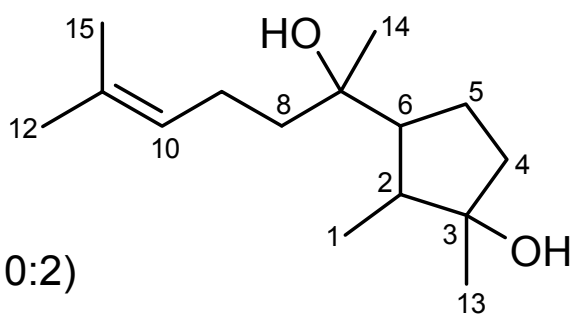

Anfärbeverhalten: pink (Anis), orange-rot (Orcin), blau-violett (Ehrlich).

ESI-MS: $\mathrm{m} / \mathrm{z}=263.3[\mathrm{M}+\mathrm{Na}]^{+}$.

IR (KBr): $\tilde{v}=3430,2965,2929,1636,1456,1379,1109,1028,921,884 \mathrm{~cm}^{-1}$ 
${ }^{1} \mathrm{H}-\mathrm{NMR}\left(600 \mathrm{MHz}, \mathrm{D}_{5}\right.$-Pyridin, $\left.35^{\circ} \mathrm{C}\right) ; \delta_{\mathrm{H}}=1.41\left(\mathrm{~d}, J=7.0 \mathrm{~Hz}, 3 \mathrm{H}, 1-\mathrm{H}_{3}\right), 1.42(\mathrm{~s}$, $3 \mathrm{H}, 14-\mathrm{H}_{3}$ ), 1.62 (brs, $\left.3 \mathrm{H}, 12-\mathrm{H}_{3}\right), 1.69$ (brs, $\left.3 \mathrm{H}, 15-\mathrm{H}_{3}\right), 1.75-1.87\left(\mathrm{~m}, 2 \mathrm{H}, 4-\mathrm{H}_{\mathrm{A}}\right.$, 8- $\left.\mathrm{H}_{\mathrm{A}}\right), 1.96-2.04\left(\mathrm{~m}, 4 \mathrm{H}, 2-\mathrm{H}, 4-\mathrm{H}_{\mathrm{B}}, 5-\mathrm{H}_{\mathrm{A}}, 8-\mathrm{H}_{\mathrm{B}}\right), 2.21-2.34\left(\mathrm{~m}, 3 \mathrm{H}, 5-\mathrm{H}_{\mathrm{B}}, 6-\mathrm{H}\right.$, 9- $\mathrm{H}_{\mathrm{A}}$ ), $2.41\left(\mathrm{~m}, 1 \mathrm{H}, 9-\mathrm{H}_{\mathrm{B}}\right), 5.00$ (brs, 1H, 3-OH), 5.02 (brs, 1H, 7-OH), 5.31 (tsept, $J=7.0,1.0 \mathrm{~Hz}, 1 \mathrm{H}, 10-\mathrm{H}) \mathrm{ppm}$.

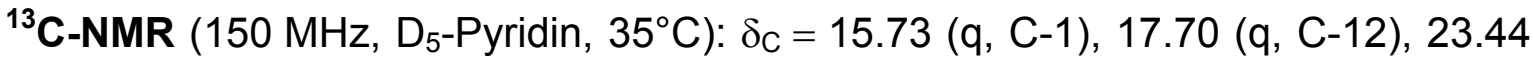
(t, C-9), 25.29 (t, C-5), 25.63 (q, C-14), 25.80 (q, C-15), 26.84 (q, C-13), 41.69 (t, C-8, C-4), 45.16 (d, C-2), 55.51 (d, C-6), 73.90 (s, C-7), 80.25 (s, C-3), 126.07 (d, C-10), 130.78 (s, C-11) ppm.

\section{Stamm WDMH 24}

\subsection{Kultivierung und Aufarbeitung}

301 L-Erlenmeyerkolben ohne Schikanen, die jeweils mit 170 mL Nährlösung G20 befüllt waren, wurden mit einem $3 \mathrm{~cm}^{2}$ großem Stück einer gut bewachsenen Agarplatte von WDMH 24 beimpft und bei $28^{\circ} \mathrm{C}$ und $120 \mathrm{spm}$ für sechs Tage inkubiert. An die Ernte schloss sich eine Aufarbeitung analog Abbildung 4.

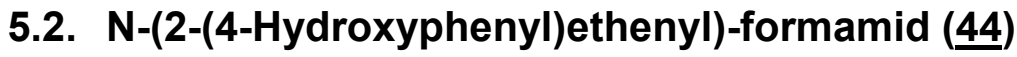

Der Kulturfiltrat-Extrakt wurde an Kieselgel $\left(\mathrm{CH}_{2} \mathrm{Cl}_{2} / \mathrm{MeOH}\right.$ 99:1 $\left.\rightarrow 32: 1\right)$, $\left(\mathrm{CH}_{2} \mathrm{Cl}_{2} / \mathrm{MeOH}\right.$ 9:1) und Sephadex LH-20 (Aceton) aufgereinigt. Man erhielt $22.8 \mathrm{mg}$ eines weißen Feststoffes.

$\mathrm{C}_{9} \mathrm{H}_{9} \mathrm{NO}_{2}(163.18)$

Rf-Wert: 0.35 (Chloroform/Methanol 9:1)

0.48 (Cyclohexan/Essigester/Methanol 5:10:2)

0.52 (Essigester/Methanol 19:1).

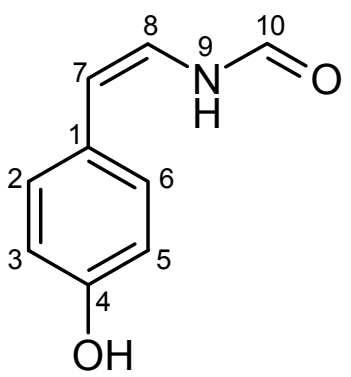

Anfärbeverhalten: bläulich braun (Anis), lila (Orcin), grau (Ehrlich).

ESI-MS: $\mathrm{m} / \mathrm{z}=186.3[\mathrm{M}+\mathrm{Na}]^{+}, 349.1[2 \mathrm{M}+\mathrm{Na}]^{+}, 162.3[\mathrm{M}-\mathrm{H}]^{-}$. 
IR $(\mathrm{KBr}): \tilde{v}=3310,3222,2800,1667,1652,1610,1589,1519,1493,1458,1403$, $1369,1273,1232,1208,1175,1106,826,599 \mathrm{~cm}^{-1}$.

UV $(\mathrm{MeOH}) \quad: \lambda_{\max }(\varepsilon)=277$ (20996), 216 (12668), 201 (12863);

$(\mathrm{MeOH} / \mathrm{HCl}) \quad: \lambda_{\max }(\varepsilon)=277$ (20079), 216 (12523), 202 (13901);

$(\mathrm{MeOH} / \mathrm{NaOH}): \lambda_{\max }(\varepsilon)=300$ (20502), 221 (12916), 205 (13662) nm.

${ }^{1} \mathrm{H}-\mathrm{NMR}\left(600 \mathrm{MHz}, \mathrm{D}_{5}\right.$-Pyridin, $\left.35^{\circ} \mathrm{C}\right): \delta_{\mathrm{H}}=5.69(\mathrm{~d}, \mathrm{~J}=10.0 \mathrm{~Hz}, 1 \mathrm{H}, 7-\mathrm{H}), 6.66(\mathrm{t}$, $J=10.5 \mathrm{~Hz}, 1 \mathrm{H}, 8-\mathrm{H}), 6.78(\mathrm{~d}, J=8.5 \mathrm{~Hz}, 2 \mathrm{H}, 3-\mathrm{H}, 5-\mathrm{H}), 7.20(\mathrm{~d}, J=8.5 \mathrm{~Hz}, 2 \mathrm{H}$, 2-H, 6-H), 8.12 (s, 1H, 10-H), 9.45 (brs, 1H, 4-OH), 9.76 (brd, J = $10.5 \mathrm{~Hz}, 1 \mathrm{H}$, 9-NH) ppm.

${ }^{13} \mathrm{C}-N M R\left(150 \mathrm{MHz}, \mathrm{D}_{5}\right.$-Pyridin, $\left.35^{\circ} \mathrm{C}\right): \delta_{\mathrm{C}}=110.77(\mathrm{~d}, \mathrm{C}-7), 115.43(2 \times \mathrm{d}, \mathrm{C}-3$, C-5), 117.78 (d, C-8), 126.08 (s, C-1), 129.49 (2 x d, C-2, C-6), 156.16 (s, C-4), 159.86 (d, C-19) ppm.

\section{Stamm WDMH 51}

\subsection{Kultivierung und Aufarbeitung}

Der Stamm WDMH 51 wurde in 1 L-Erlenmeyerkolben ohne Schikanen kultiviert. Diese waren jeweils mit $170 \mathrm{~mL}$ Nährlösung G20 befüllt und wurden mit einem $3 \mathrm{~cm}^{2}$ großem Stück einer gut bewachsenen Agarplatte beimpft. Nach sechs Tagen Inkubation bei $28{ }^{\circ} \mathrm{C}$ und 120 spm wurde der 5 L-Ansatz geerntet und der Standard-Aufarbeitung analog Abbildung 4 unterzogen.

\subsection{Isolierung}

Die Aufreinigung des eingeengten Myzel-Extraktes ist in Abbildung 11 dargestellt. 


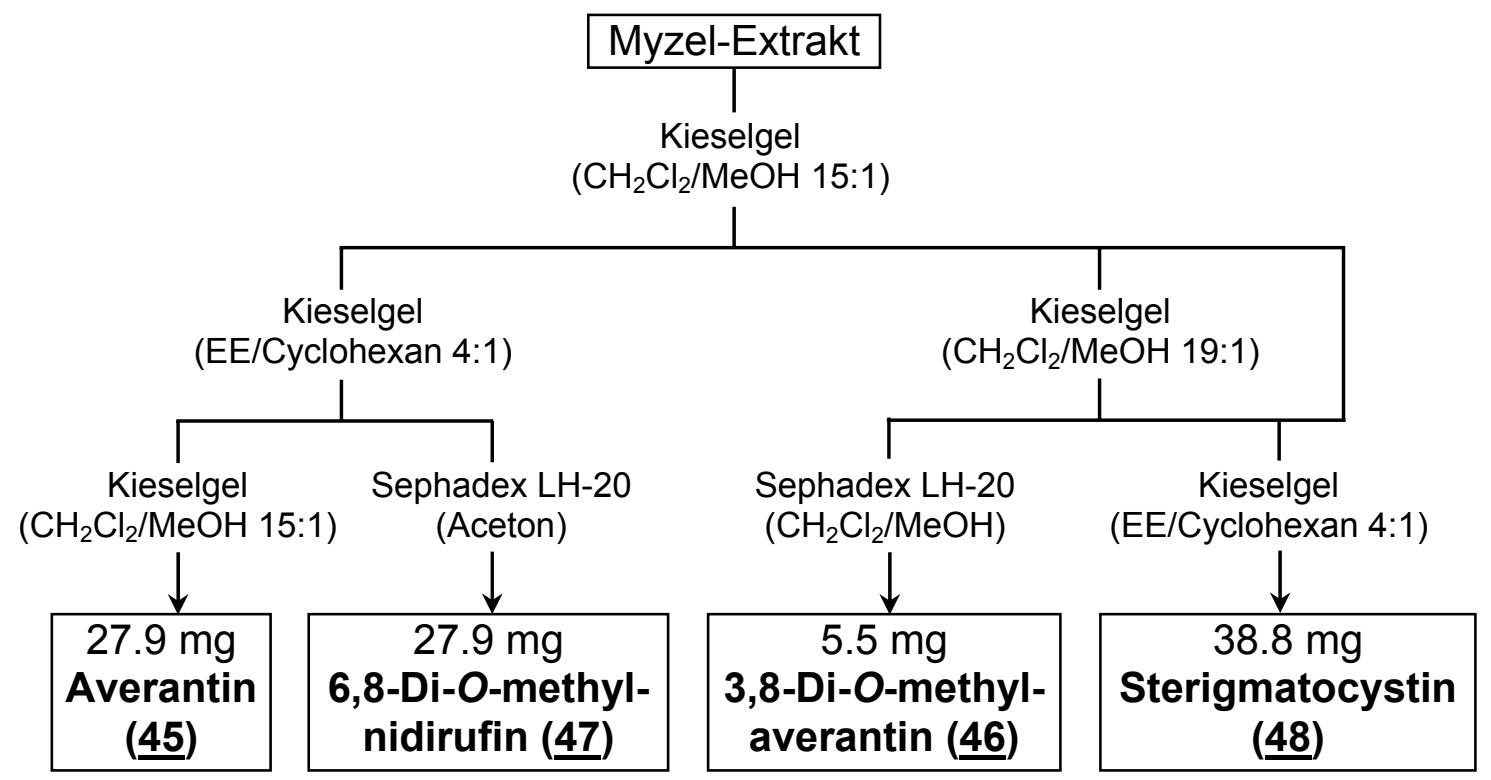

Abbildung 11: Isolierung der Sekundärmetabolite aus WDMH51.

\subsection{Charakterisierung der Metabolite}

Averantin (45)

$\mathrm{C}_{20} \mathrm{H}_{20} \mathrm{O}_{7}$ (372.38)

$\mathbf{R}_{\mathrm{f}}$-Wert: 0.46 (Chloroform/Methanol 9:1)<smiles>CCCCCCC(O)c1c(O)cc2c(c1O)C(=O)c1c(O)cc(O)cc1C2=O</smiles>

0.64 (Cyclohexan/Essigester/Methanol 5:10:2)

0.63 (Essigester/Methanol 19:1).

Anfärbeverhalten: orange (Anis), orange (Orcin), orange-rot (Ehrlich).

$[\alpha]_{D}^{20}=-135^{\circ}\left(\mathrm{c}=1.0 \mathrm{mg} / \mathrm{mL}\right.$ in Methanol), $\left(\mathrm{Lit}_{.}^{54}[\alpha]_{D}^{20}=-178^{\circ}(0.37 \mathrm{~g} / \mathrm{L}, \mathrm{EtOH})\right)$.

ESI-MS: $\mathrm{m} / \mathrm{z}=395.4[\mathrm{M}+\mathrm{Na}]^{+}, 417.3[\mathrm{M}-\mathrm{H}+2 \mathrm{Na}]^{+}, 767.4[2 \mathrm{M}+\mathrm{Na}]^{+}, 371.3[\mathrm{M}-\mathrm{H}]^{-}$, $765.3[2 \mathrm{M}-2 \mathrm{H}+\mathrm{Na}]^{-}$.

IR (KBr): $\widetilde{v}=3340,2929,2857,1617,1469,1402,1340,1326,1286,1264,1207$, $1179,782,622 \mathrm{~cm}^{-1}$. 
UV $(\mathrm{MeOH}) \quad: \lambda_{\max }(\varepsilon)=222$ (30185), 263 (16443), 293 (26270), 311 (15283), 454 (8480);

$(\mathrm{MeOH} / \mathrm{HCl}) \quad: \lambda_{\max }(\varepsilon)=222$ (31069), $254 \mathrm{sh}$ (13861), 266 sh (15986), 292 (29819), 322 (9046), 452 (9524);

$(\mathrm{MeOH} / \mathrm{NaOH}): \lambda_{\max }(\varepsilon)=205$ (22967), 230 (22497), 245 sh (15459), 264 (14197), 319 (50322), 395 (7523), 538 (7982) nm.

CD (MeOH): $\lambda_{\max }[\Theta]=211$ (11480), 227 (-11284), 264 (3105), 290 (-6684), 321 (6724), $386(-4491) \mathrm{nm}$.

${ }^{1} \mathrm{H}-N M R\left(500 \mathrm{MHz}, \mathrm{D}_{6}\right.$-Aceton): $\delta_{\mathrm{H}}=0.90\left(\mathrm{t}, J=7.0 \mathrm{~Hz}, 3 \mathrm{H}, 6^{\prime}-\mathrm{H}_{3}\right), 1.35(\mathrm{~m}, 2 \mathrm{H}$, $\left.5^{\prime}-\mathrm{H}_{2}\right), 1.36\left(\mathrm{~m}, 2 \mathrm{H}, 4^{\prime}-\mathrm{H}_{2}\right), 1.51\left(\mathrm{~m}, 1 \mathrm{H}, 3^{\prime}-\mathrm{H}_{\mathrm{A}}\right), 1.57\left(\mathrm{~m}, 1 \mathrm{H}, 3^{\prime}-\mathrm{H}_{\mathrm{B}}\right), 1.85(\mathrm{~m}, 2 \mathrm{H}$, $\left.2^{\prime}-\mathrm{H}_{2}\right), 5.41$ (ddd, $\left.J=8.0,4.5,2.0 \mathrm{~Hz}, 1 \mathrm{H}, 1^{\prime}-\mathrm{H}\right), 6.60(\mathrm{~m}, 1 \mathrm{H}, 7-\mathrm{H}), 7.09(\mathrm{~m}, 1 \mathrm{H}$, 4-H), 7.18 (m, 1H, 5-H), 12.17 (brs, 1H, 8-OH), 12.73 (brs, 1H, 1-OH) ppm.

${ }^{13}$ C-NMR (125.71 MHz, D 6 -Aceton): $\delta_{\mathrm{C}}=14.27$ (q, C-6'), 23.21 (t, C-5'), 25.63 (t, C-3'), 32.28 (t, C-4'), 36.93 (t, C-2'), 69.95 (d, C-1'), 108.89 (d, C-7), 109.39 (s, C-13), 109.65 (d, C-5), 109.99 (s, C-12), 110.42 (d, C-4), 122.23 (s, C-2), 134.38 (s, C-14), 136.22 (s, C-11), 161.67 (s, C-1), 165.08 (s, C-3), 165.91 (s, C-8), 166.09 (s, C-6), 181.73 (s, C-10), 190.46 (s, C-9) ppm.

\section{3,6-Di-O-methyl-averantin (느)}

$\mathrm{C}_{22} \mathrm{H}_{24} \mathrm{O}_{7}(400.43)$

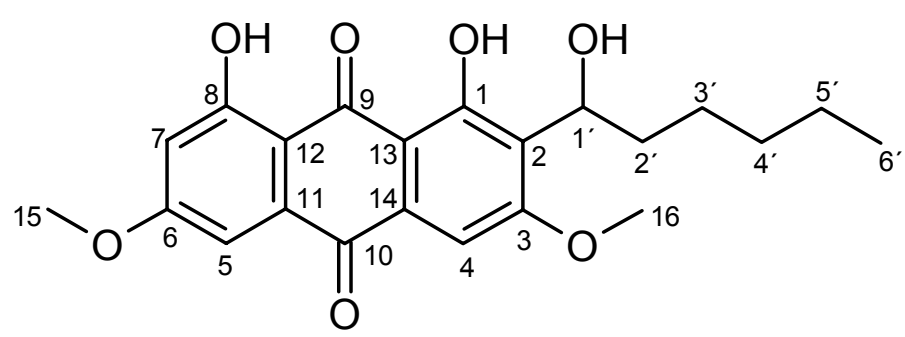

$\mathbf{R}_{\mathbf{f}}$-Wert: 0.65 (Chloroform/Methanol 9:1)

0.61 (Cyclohexan/Essigester/Methanol 5:10:2)

0.60 (Essigester/Methanol 19:1).

Anfärbeverhalten: orange (Anis), pink (Orcin), orange (Ehrlich).

$[\alpha]_{D}^{20}=-104^{\circ}(\mathrm{c}=1.0 \mathrm{mg} / \mathrm{mL}$ in Methanol). 
ESI-MS: $\mathrm{m} / \mathrm{z}=423.4[\mathrm{M}+\mathrm{Na}]^{+}, 823.3[2 \mathrm{M}+\mathrm{Na}]^{+}, 399.6[\mathrm{M}-\mathrm{H}]^{-}, 821.4[2 \mathrm{M}-\mathrm{H}+\mathrm{Na}]^{-}$.

IR (KBr): $\widetilde{v}=3425,2930,2857,1618,1595,1559,1484,1458,1430,1397,1325$, $1258,1212,1161,1134,1056,975 \mathrm{~cm}^{-1}$.

UV $(\mathrm{MeOH}) \quad: \lambda_{\max }(\varepsilon)=222$ (31141), 249 sh (12814), 285 (25843), 315 sh (9703), 443 (7205);

$(\mathrm{MeOH} / \mathrm{HCl}) \quad: \lambda_{\max }(\varepsilon)=223$ (32124), 249 sh (12537), 285 (27011), 319 sh (7639), 443 (7479);

$(\mathrm{MeOH} / \mathrm{NaOH}): \lambda_{\max }(\varepsilon)=217$ (29339), 244 sh (15186), 260 (19489), 310 (26068), 493 (5409) nm.

CD $(\mathrm{MeOH}): \lambda_{\max }[\Theta]=204$ (12701), 207 (10004), 209 (11405), 214 (4809), 217 (7730), 223 (661), 225 (1682), 232 (-6543), 263 (1776), 283 (-6143), 311 (4800), $388(-3362) \mathrm{nm}$.

${ }^{1} \mathrm{H}-\mathrm{NMR}\left(500 \mathrm{MHz}, \mathrm{CDCl}_{3}\right): \delta_{\mathrm{H}}=0.90\left(\mathrm{t}, J=7.0 \mathrm{~Hz}, 3 \mathrm{H}, 6^{\prime}-\mathrm{H}_{3}\right), 1.32(\mathrm{~m}, 2 \mathrm{H}$, $\left.5^{\prime}-\mathrm{H}_{2}\right), 1.33\left(\mathrm{~m}, 2 \mathrm{H}, 4^{\prime}-\mathrm{H}_{2}\right), 1.43\left(\mathrm{~m}, 1 \mathrm{H}, 3^{\prime}-\mathrm{H}_{\mathrm{A}}\right), 1.55\left(\mathrm{~m}, 1 \mathrm{H}, 3^{\prime}-\mathrm{H}_{\mathrm{B}}\right), 1.76(\mathrm{~m}, 1 \mathrm{H}$, $\left.2^{\prime}-\mathrm{H}_{\mathrm{A}}\right), 1.84\left(\mathrm{~m}, 1 \mathrm{H}, 2^{\prime}-\mathrm{H}_{\mathrm{B}}\right), 4.00\left(\mathrm{~s}, 3 \mathrm{H}, 15-\mathrm{H}_{3}\right), 4.02\left(\mathrm{~s}, 3 \mathrm{H}, 16-\mathrm{H}_{3}\right), 5.43$ (dd, $\left.J=8.0,4.5 \mathrm{~Hz}, 1 \mathrm{H}, 1^{\prime}-\mathrm{H}\right), 6.77$ (d, J = 2.5 Hz, 1H, 7-H), 6.85 (s, 1H, 4-H), 7.43 (d, $J=2.5 \mathrm{~Hz}, 1 \mathrm{H}, 5-\mathrm{H}), 9.66$ (brs, 1H, 8-OH), 13.60 (s, 1H, 1-OH) ppm.

${ }^{13}$ C-NMR (125.71 MHz, $\mathrm{CDCl}_{3}$ ): $\delta_{\mathrm{C}}=14.02$ (q, C-6'), 22.56 (t, C-5'), 25.05 (t, C-3'), 31.57 (t, C-4'), 35.47 (t, C-2'), 56.04 (q, C-15), 56.52 (q, C-16), 70.03 (d, C-1'), 104.16 (d, C-5), 104.75 (d, C-7), 108.90 (d, C-4), 109.77 (s, C-13), 114.84 (s, C-12), 121.49 (s, C-2), 132.56 (s, C-14), 137.34 (s, C-11), 161.19 (s, C-1), 162.55 (s, C-3), 162.68 (s, C-8), 165.19 (s, C-6), 181.90 (s, C-10), 187.08 (s, C-9) ppm.

\section{6,8-Di-O-methyl-nidirufin (47)}

$\mathrm{C}_{22} \mathrm{H}_{20} \mathrm{O}_{8}(412.40)$

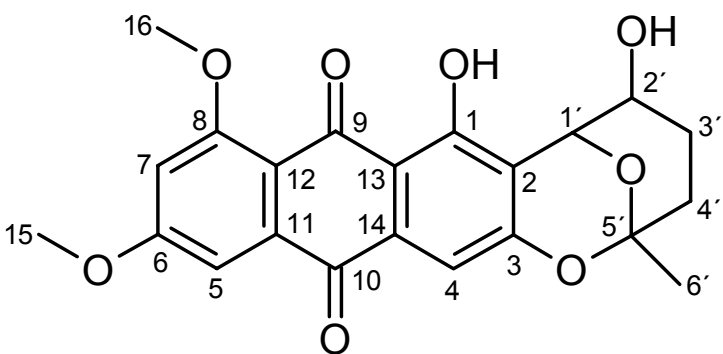


$\mathbf{R}_{\mathbf{f}}$-Wert: 0.58 (Chloroform/Methanol 9:1)

0.51 (Cyclohexan/Essigester/Methanol 5:10:2)

0.50 (Essigester/Methanol 19:1).

Anfärbeverhalten: orange (Anis), pink (Orcin), orange (Ehrlich).

$[\alpha]_{D}^{20}=-56^{\circ}\left(\mathrm{c}=1.0 \mathrm{mg} / \mathrm{mL}\right.$ in Methanol), $\left(\right.$ Lit. $\left.^{54}[\alpha]_{D}^{25}=-77^{\circ}\left(0.15 \mathrm{~g} / \mathrm{L}, \mathrm{CHCl}_{3}\right)\right)$.

ESI-MS: $\mathrm{m} / \mathrm{z}=435.6[\mathrm{M}+\mathrm{Na}]^{+}, 847.3[2 \mathrm{M}+\mathrm{Na}]^{+}, 845.6[2 \mathrm{M}-2 \mathrm{H}+\mathrm{Na}]^{-}$.

IR $(\mathrm{KBr}): \tilde{v}=3446,2937,1672,1622,1596,1559,1481,1456,1438,1392,1341$, $1326,1291,1264,1241,1209,1161,1055,1043,842 \mathrm{~cm}^{-1}$.

UV $(\mathrm{MeOH}) \quad: \lambda_{\max }(\varepsilon)=224$ (33725), 251 sh (13756), 286 (28244), 317 sh (8778), 441 (7747);

$(\mathrm{MeOH} / \mathrm{HCl}) \quad: \lambda_{\max }(\varepsilon)=224$ (33784), 250 sh (13020), 286 (29012), 441 (7812);

$(\mathrm{MeOH} / \mathrm{NaOH}): \lambda_{\max }(\varepsilon)=223$ (32350), 286 (25071), 317 sh (10369), 441 (7073) $\mathrm{nm}$.

CD $(\mathrm{MeOH}): \lambda_{\max }[\Theta]=204$ (2544), 215 (10154), 219 (6779), 221 (8444), 232 (-13545), 245 (5863), 252 (3666), 263 (5184), 289 (-9341), 311 (-26), 361 (-5115) $\mathrm{nm}$.

${ }^{1} \mathrm{H}-N M R\left(500 \mathrm{MHz}, \mathrm{CDCl}_{3}\right): \delta_{\mathrm{H}}=1.65\left(\mathrm{~s}, 3 \mathrm{H}, 6^{\prime}-\mathrm{H}_{3}\right), 1.76\left(\mathrm{~m}, 2 \mathrm{H}, 3^{\prime}-\mathrm{H}_{2}\right), 1.95$ (ddd, $\left.J=14.0,4.0,3.0 \mathrm{~Hz}, 1 \mathrm{H}, 4^{\prime}-\mathrm{H}_{\mathrm{A}}\right), 2.21\left(\mathrm{~m}, 1 \mathrm{H}, 4^{\prime}-\mathrm{H}_{\mathrm{B}}\right), 4.00\left(\mathrm{~s}, 3 \mathrm{H}, 15-\mathrm{H}_{3}\right)$, $4.05\left(\mathrm{~s}, 3 \mathrm{H}, 16-\mathrm{H}_{3}\right), 4.10\left(\mathrm{q}, J=2.5 \mathrm{~Hz}, 1 \mathrm{H}, 2^{\prime}-\mathrm{H}\right), 5.27$ (d, J = $\left.2.5 \mathrm{~Hz}, 1 \mathrm{H}, 1^{\prime}-\mathrm{H}\right)$, $6.79(\mathrm{~d}, J=2.5 \mathrm{~Hz}, 1 \mathrm{H}, 7-\mathrm{H}), 7.22(\mathrm{~s}, 1 \mathrm{H}, 4-\mathrm{H}), 7.46(\mathrm{~d}, J=2.5 \mathrm{~Hz}, 1 \mathrm{H}, 5-\mathrm{H})$, $13.60(\mathrm{~s}, 1 \mathrm{H}, 1-\mathrm{OH}) \mathrm{ppm}$.

${ }^{13}$ C-NMR (125.71 MHz, $\left.\mathrm{CDCl}_{3}\right): \delta_{\mathrm{C}}=23.18$ (t, C-3'), 27.60 (q, C-6'), 30.75 (t, C-4'), 56.03 (q, C-15), 56.62 (q, C-16), 65.23 (d, C-2'), 71.32 (d, C-1'), 100.84 (s, C-5'), 104.08 (d, C-5), 104.86 (d, C-7), 107.24 (d, C-4), 110.01 (s, C-13), 114.73 (s, C-2), 115.12 (s, C-12), 132.95 (s, C-14), 137.47 (s, C-11), 159.21 (s, C-3), 159.76 (s, C-1), 162.87 (s, C-8), 165.06 (s, C-6), 182.49 (s, C-10), 186.71 (s, C-9) ppm. 


\section{Sterigmatocystin (푸)}

$\mathrm{C}_{18} \mathrm{H}_{12} \mathrm{O}_{6}(324.29)$

$\mathbf{R}_{\mathbf{f}}$-Wert: 0.73 (Chloroform/Methanol 9:1)

0.62 (Cyclohexan/Essigester/Methanol 5:10:2) 0.62 (Essigester/Methanol 19:1).

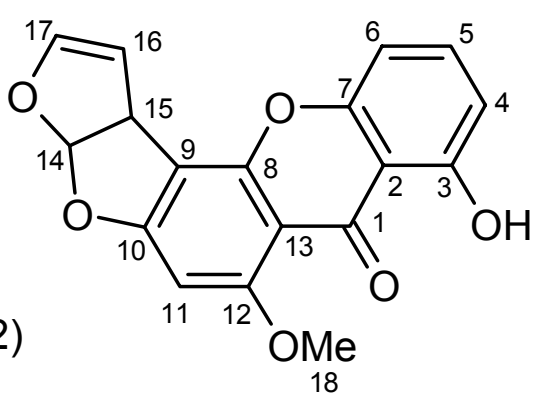

Anfärbeverhalten: gelblich braun (Anis), grün-rot (Orcin), helles lila (Ehrlich).

$[\alpha]_{D}^{20}=-314^{\circ}\left(\mathrm{c}=1.0 \mathrm{mg} / \mathrm{mL}\right.$ in $\left.\mathrm{CHCl}_{3}\right),\left(\mathrm{Lit}^{54}[\alpha]_{D}^{20}=-398^{\circ}\left(1.0 \mathrm{~g} / \mathrm{L}, \mathrm{CHCl}_{3}\right)\right)$.

ESI-MS: $\mathrm{m} / \mathrm{z}=347.6[\mathrm{M}+\mathrm{Na}]^{+}, 671.3[2 \mathrm{M}+\mathrm{Na}]^{+}$.

IR $(\mathrm{KBr}): \widetilde{v}=3425,3099,2926,1649,1628,1587,1483,1459,1268,1233,1127$, 1093, 1060, $972 \mathrm{~cm}^{-1}$.

UV $(\mathrm{MeOH}) \quad: \lambda_{\max }(\varepsilon)=203 \quad(24551), \quad 234$ sh $(27480), \quad 245 \quad(30304), \quad 326$ (13296);

$(\mathrm{MeOH} / \mathrm{HCl}) \quad: \lambda_{\max }(\varepsilon)=203 \quad(24551), 236$ sh (28618), 245 (30852), 326 (13574);

$(\mathrm{MeOH} / \mathrm{NaOH}): \lambda_{\max }(\varepsilon)=204$ (22961), 236 sh (29713), 243 (31652), 326 (13572) $\mathrm{nm}$.

${ }^{1} \mathrm{H}-\mathrm{NMR}\left(500 \mathrm{MHz}, \mathrm{CDCl}_{3}\right): \delta_{\mathrm{H}}=3.98\left(\mathrm{~s}, 3 \mathrm{H}, 18-\mathrm{H}_{3}\right), 4.77(\mathrm{dt}, J=7.0,2.0 \mathrm{~Hz}, 1 \mathrm{H}$, $15-\mathrm{H}), 5.44(\mathrm{t}, J=2.5 \mathrm{~Hz}, 1 \mathrm{H}, 16-\mathrm{H}), 6.41(\mathrm{~s}, 1 \mathrm{H}, 11-\mathrm{H}), 6.50(\mathrm{t}, J=2.5 \mathrm{~Hz}, 1 \mathrm{H}$, $17-\mathrm{H}), 6.74$ (d, J=8.5 Hz, 1H, 4-H), 6.80 (d, J=7.5 Hz, 1H, 6-H), 6.81 (d, $J=7.0 \mathrm{~Hz}, 1 \mathrm{H}, 14-\mathrm{H}), 7.48(\mathrm{t}, J=8.5 \mathrm{~Hz}, 1 \mathrm{H}, 5-\mathrm{H}), 13.22(\mathrm{~s}, 1 \mathrm{H}, 3-\mathrm{OH}) \mathrm{ppm}$.

${ }^{13}$ C-NMR (125.71 MHz, $\mathrm{CDCl}_{3}$ ): $\delta_{\mathrm{C}}=47.95$ (d, C-15), 56.69 (q, C-18), 90.40 (d, C-11), 102.45 (d, C-16), 105.78 (s, C-13), 105.82 (d, C-6), 106.44 (s, C-9), 108.84 (s, C-2), 111.12 (d, C-4), 113.20 (d, C-14), 135.58 (d, C-5), 145.28 (d, C-17), 153.88 (s, C-8), 154.83 (s, C-7), 162.19 (s, C-3), 163.16 (s, C-12), 164.49 (s, C-10), 181.21 (s, C-1) ppm. 


\section{Stamm WDMH 28}

\subsection{Kultivierung und Aufarbeitung}

301 L-Erlenmeyerkolben ohne Schikanen, befüllt mit jeweils $170 \mathrm{~mL}$ Nährlösung 1158 wurden mit je einem $3 \mathrm{~cm}^{2}$ großem Stück einer gut bewachsenen Agarplatte des Stamm WDMH 28 beimpft und bei $28^{\circ} \mathrm{C}$ und 120 spm inkubiert. Nach sechstägiger Kultivierung wurden die Kolben geerntet und nach dem Schema in Abbildung 4 aufgearbeitet.

\subsection{Isolierung}

Der Kulturfiltrat-Extrakt wurde an Kieselgel $\left(\mathrm{CH}_{2} \mathrm{Cl}_{2} / \mathrm{MeOH} 20: 1 \rightarrow 4: 1\right)$ säulenchromatographiert. Weitere Aufreinigung der 2. Fraktion an Sephadex LH-20 $\left(\mathrm{CH}_{2} \mathrm{Cl}_{2} / \mathrm{MeOH}\right.$ 1:1) und Kieselgel (EE/Cyclohexan 4:1) lieferte ein Substanzgemisch aus zwei Verbindungen, das präparativ an der HPLC aufgetrennt wurde (HPLC 3, Säule 4, Programm 5). Man erhielt $128.3 \mathrm{mg} \underline{\mathbf{5 6}}$ und $35.6 \mathrm{mg} \underline{\mathbf{6 0}}$.

Die 3. Fraktion der Rohprodukt-Säule reinigte man an Kieselgel (Cyclohexan/EE/MeOH 5:10:1) und Sephadex $\mathrm{LH}-20\left(\mathrm{CH}_{2} \mathrm{Cl}_{2} / \mathrm{MeOH} 1: 1\right.$ und Aceton) auf. Diese Auftrennungen ergaben $5.3 \mathrm{mg} \underline{\mathbf{6 1}}$ und eine Mischfraktion. Abschließende Chromatographie über die präparative HPLC (HPLC 3, Säule 5, Programm 7) führte zu zwei weiteren Reinsubstanzen: $8.2 \mathrm{mg} \underline{\mathbf{5 1}}$ und $6.0 \mathrm{mg} \underline{\mathbf{5 2}}$. Säulenchromatographie der 6. Rohprodukt-Fraktion an Kieselgel (EE/MeOH 19:1), Sephadex $\mathrm{LH}-20\left(\mathrm{CH}_{2} \mathrm{Cl}_{2} / \mathrm{MeOH} 1: 1\right)$ brachten $14.9 \mathrm{mg} \underline{\mathbf{5 0}} \mathrm{zu}$ Tage.

\subsection{Charakterisierung der Metabolite}

$\alpha$-Acetylorcinol (무)

$\mathrm{C}_{9} \mathrm{H}_{10} \mathrm{O}_{3}(166.18)$

Rf-Wert: 0.24 (Chloroform/Methanol 9:1)<smiles>CC(=O)Cc1cc(O)cc(O)c1</smiles>

0.47 (Cyclohexan/Essigester/Methanol 5:10:2)

0.49 (Essigester/Methanol 19:1).

Anfärbeverhalten: orange-braun (Anis). 
EI-MS: $\mathrm{m} / \mathrm{z}(\%)=166.1(100)[\mathrm{M}]^{+}, 124.1(65), 123.1(70)\left[\mathrm{M}-\mathrm{COCH}_{3}\right]^{+}, 107.1(38)$, $43(72)\left[\mathrm{COCH}_{3}\right]^{+}$.

IR $(\mathrm{KBr}): \tilde{v}=3390,2977,2914,1704,1609,1461,1353,1302,1158,1026,996$, $843,706,581 \mathrm{~cm}^{-1}$.

UV $(\mathrm{MeOH}) \quad: \lambda_{\max }(\varepsilon)=202$ (18265), 218 sh (7359), 282 (1629);

$(\mathrm{MeOH} / \mathrm{HCl}) \quad: \lambda_{\max }(\varepsilon)=201$ (15157), 282 (1355);

$(\mathrm{MeOH} / \mathrm{NaOH}): \lambda_{\max }(\varepsilon)=205$ (17571), $287(2101) \mathrm{nm}$.

${ }^{1} \mathrm{H}-\mathrm{NMR}\left(600 \mathrm{MHz}, \mathrm{CD}_{3} \mathrm{OD}\right): \delta_{\mathrm{H}}=2.10\left(\mathrm{~s}, 3 \mathrm{H}, 9-\mathrm{H}_{3}\right), 3.528\left(\mathrm{~s}, 2 \mathrm{H}, 7-\mathrm{H}_{2}\right), 6.15(\mathrm{~s}$, $3 \mathrm{H}, 2-\mathrm{H}, 4-\mathrm{H}, 6-\mathrm{H}) \mathrm{ppm}$.

${ }^{13} \mathrm{C}-\mathrm{NMR}\left(150 \mathrm{MHz}, \mathrm{CD}_{3} \mathrm{OD}\right): \delta_{\mathrm{C}}=28.98$ (q, C-9), 51.57 (t, C-7), 102.21 (d, C-4), 108.94 (d, C-2, C-6), 137.84 (s, C-1), 159.75 (s, C-3, C-5), 209.66 (s, C-8) ppm.

Isoscleron (1)

$\mathrm{C}_{10} \mathrm{H}_{10} \mathrm{O}_{3}(178.19)$

$\mathbf{R}_{\mathbf{f}}$-Wert: 0.46 (Chloroform/Methanol 9:1)<smiles>O=C1CCC(O)c2cccc(O)c21</smiles>

0.50 (Cyclohexan/Essigester/Methanol 5:10:2) 0.47 (Essigester/Methanol 19:1).

Anfärbeverhalten: blau (Anis).

$[\alpha]_{D}^{20}=+9^{\circ}(\mathrm{c}=1.0 \mathrm{mg} / \mathrm{mL}$ in Methanol $)$.

EI-MS: $\mathrm{m} / \mathrm{z}(\%)=178.1(100)[\mathrm{M}]^{+}, 160.1(12)\left[\mathrm{M}-\mathrm{H}_{2} \mathrm{O}\right]^{+}, 150.1(20), 121.1(57)$.

IR (KBr): $\tilde{v}=3420,3346,3251,2971,2945,2878,1637,1575,1455,1334,1308$, $1256,1198,1159,1071,985,894,845,809,747 \mathrm{~cm}^{-1}$.

UV $(\mathrm{MeOH}) \quad: \lambda_{\max }(\varepsilon)=214$ (15124), 258 (7019), 331 (2231);

$(\mathrm{MeOH} / \mathrm{HCl}) \quad: \lambda_{\max }(\varepsilon)=214$ (14985), 259 (7047), 330 (2287); 
$(\mathrm{MeOH} / \mathrm{NaOH}): \lambda_{\max }(\varepsilon)=201$ (11697), 214 (16614), $258(8817), 332(4048) \mathrm{nm}$.

CD $(\mathrm{MeOH}): \lambda_{\max }[\Theta]=213$ (37863), $231(-2655), 235(-956), 259(-11265) \mathrm{nm}$.

${ }^{1} \mathrm{H}-\mathrm{NMR}\left(600 \mathrm{MHz}, \mathrm{CDCl}_{3}\right): \delta_{\mathrm{H}}=2.14$ (dddd, $J=13.0,8.0,8.0,5.0 \mathrm{~Hz}, 1 \mathrm{H}, 3-\mathrm{H}_{\mathrm{A}}$ ), 2.29 (dddd, $J=13.0,8.5,4.5,4.0 \mathrm{~Hz}, 1 \mathrm{H}, 3-\mathrm{H}_{\mathrm{B}}$ ), 2.60 (ddd, $J=18.0,8.5,5.0 \mathrm{~Hz}$, $1 \mathrm{H}, 2-\mathrm{H}_{\mathrm{A}}$ ), 2.95 (ddd, $J=18.0,8.0,4.5 \mathrm{~Hz}, 1 \mathrm{H}, 2-\mathrm{H}_{\mathrm{B}}$ ), 4.87 (dd, $J=7.5,4.0 \mathrm{~Hz}$, $1 \mathrm{H}, 4-\mathrm{H}), 6.87(\mathrm{dd}, J=8.0,0.5 \mathrm{~Hz}, 1 \mathrm{H}, 7-\mathrm{H}), 6.98(\mathrm{~d}, J=7.5 \mathrm{~Hz}, 1 \mathrm{H}, 5-\mathrm{H}), 7.45(\mathrm{t}$, $J=8.0 \mathrm{~Hz}, 1 \mathrm{H}, 6-\mathrm{H}), 12.37$ (s, 1H, 8-OH) ppm.

${ }^{13} \mathrm{C}-\mathrm{NMR}\left(150 \mathrm{MHz}, \mathrm{CDCl}_{3}\right): \delta_{\mathrm{C}}=31.13$ (t, C-3), 34.52 (t, C-2), 67.59 (d, C-4), 115.18 (s, C-8a), 117.39 (d, C-5), 117.65 (d, C-7), 136.95 (d, C-6), 145.85 (s, C-4a), 162.58 (s, C-8), 204.32 (s, C-1) ppm.

\section{6-Methoxyisoscleron ( $\underline{52})$}

$\mathrm{C}_{11} \mathrm{H}_{12} \mathrm{O}_{4}(208.21)$

Rf-Wert: 0.48 (Chloroform/Methanol 9:1)

0.49 (Cyclohexan/Essigester/Methanol 5:10:2)<smiles>COc1cc(O)c2c(c1)C(=O)CCC2=O</smiles>
0.47 (Essigester/Methanol 19:1).

Anfärbeverhalten: violett (Anis).

$[\alpha]_{D}^{20}=+20^{\circ}(\mathrm{c}=1.0 \mathrm{mg} / \mathrm{mL}$ in Methanol $)$.

EI-MS: $\mathrm{m} / \mathrm{z}(\%)=208.1(100)[\mathrm{M}]^{+}, 180.1(22), 152.1(58), 151.1(85)$.

IR (KBr): $\tilde{v}=3428,3288,3174,2934,2864,1632,1489,1431,1292,1204,1164$, $1039,839 \mathrm{~cm}^{-1}$.

UV (MeOH) $: \lambda_{\max }(\varepsilon)=218$ (5739), 214 (5799), 231 sh (3748), 239 sh (3241), 279 (5526), 316 sh (2252);

$(\mathrm{MeOH} / \mathrm{HCl}) \quad: \lambda_{\max }(\varepsilon)=214$ (6576), 217 (12601), 231 sh (4381), 239 sh (3730), 279 (5763), 317 sh (2502); 
$(\mathrm{MeOH} / \mathrm{NaOH}): \lambda_{\max }(\varepsilon)=201$ (6978), 204 (7045), 206 (6779), 210 (6750), 231 sh (4599), 236 sh (4110), 279 (5875), 317 sh (2584) nm.

CD (MeOH): $\lambda_{\max }[\Theta]=212$ (14792), 238 (-2617), 245 (-759), 278 (-5738), 303 (2061) 308 (1698), 311 (1896), 324 (452), 327 (636) nm.

${ }^{1} \mathrm{H}-\mathrm{NMR}\left(600 \mathrm{MHz}, \mathrm{CDCl}_{3}\right): \delta_{\mathrm{H}}=2.10\left(\mathrm{~m}, 1 \mathrm{H}, 3-\mathrm{H}_{\mathrm{A}}\right), 2.28(\mathrm{dddd}, \mathrm{J}=13.0,8.5,5.0$, $4.0 \mathrm{~Hz}, 1 \mathrm{H}, 3-\mathrm{H}_{\mathrm{B}}$ ), 2.58 (ddd, $J=18.0,9.0,5.0 \mathrm{~Hz}, 1 \mathrm{H}, 2-\mathrm{H}_{\mathrm{A}}$ ), 2.87 (ddd, $J=18.0$, 7.5, $\left.4.5 \mathrm{~Hz}, 1 \mathrm{H}, 2-\mathrm{H}_{\mathrm{B}}\right), 3.82\left(\mathrm{~s}, 3 \mathrm{H}, 6-\mathrm{OCH}_{3}\right), 4.80$ (dd, J=8.0, $\left.4.0 \mathrm{~Hz}, 1 \mathrm{H}, 4-\mathrm{H}\right)$, $6.33(\mathrm{~d}, J=2.5 \mathrm{~Hz}, 1 \mathrm{H}, 7-\mathrm{H}), 6.57$ (dd, $J=2.5,1.0 \mathrm{~Hz}, 1 \mathrm{H}, 5-\mathrm{H}), 12.81(\mathrm{~s}, 1 \mathrm{H}$, 8-OH) ppm.

${ }^{13} \mathrm{C}-N M R\left(150 \mathrm{MHz}, \mathrm{CDCl}_{3}\right): \delta_{\mathrm{C}}=31.35$ (t, C-3), 34.41 (t, C-2), $55.62\left(\mathrm{q}, \mathrm{OCH}_{3}\right)$, 67.92 (d, C-4), 100.17 (d, C-7), 105.61 (d, C-5), 109.65 (s, C-8a), 147.85 (s, C-4a), 165.68 (s, C-8), 166.44 (s, C-6), 202.01 (s, C-1) ppm.

\section{6-Methoxyparvulenon (므)}

$\mathrm{C}_{13} \mathrm{H}_{16} \mathrm{O}_{5}(252.27)$

$\mathbf{R}_{\mathbf{f}}$-Wert: 0.31 (Chloroform/Methanol 9:1)

0.33 (Cyclohexan/Essigester/Methanol 5:10:2)<smiles>COc1cc2c(c(O)c1C(C)O)C(=O)CCC2O</smiles>
0.31 (Essigester/Methanol 19:1).

Anfärbeverhalten: blaugrau (Anis).

$[\alpha]_{D}^{20}=+15^{\circ}(\mathrm{c}=1.0 \mathrm{mg} / \mathrm{mL}$ in Methanol).

ESI-MS: $\mathrm{m} / \mathrm{z}=275.0[\mathrm{M}+\mathrm{Na}]^{+}, 509.0\left[2 \mathrm{M}-\mathrm{H}_{2} \mathrm{O}+\mathrm{Na}\right]^{+}, 994.9\left[4 \mathrm{M}-2 \mathrm{H}_{2} \mathrm{O}+\mathrm{Na}\right]^{+}, 251.3$ $[\mathrm{M}-\mathrm{H}]^{-}, 485.2\left[2 \mathrm{M}-\mathrm{H}_{2} \mathrm{O}-\mathrm{H}\right]^{-}$.

El-MS $(70 \mathrm{eV}): \mathrm{m} / \mathrm{z}(\%)=252.1(8)[\mathrm{M}]^{+}, 237.1(100)\left[\mathrm{M}-\mathrm{CH}_{3}\right]^{+}, 219.1(24)[\mathrm{M}-$ $\left.\mathrm{CH}_{3}-\mathrm{H}_{2} \mathrm{O}\right]^{+}$.

IR $(\mathrm{KBr}): \widetilde{v}=3412,2950,1620,1486,1409,1287,1211,1153,1080,840 \mathrm{~cm}^{-1}$ 
UV $(\mathrm{MeOH}) \quad: \lambda_{\max }(\varepsilon)=223$ (15691), 237 sh (8881), 285 (13677), 319 sh (3939);

$(\mathrm{MeOH} / \mathrm{HCl}) \quad: \lambda_{\max }(\varepsilon)=223$ (15092), $238 \mathrm{sh}$ (7509), 284 (13496), $317 \mathrm{sh}$ (3142), 337 sh (2312);

$(\mathrm{MeOH} / \mathrm{NaOH}): \lambda_{\max }(\varepsilon)=203$ (13578), 224 (14877), 236 sh (11064), 284 (13075), $332 \mathrm{sh}(3732) \mathrm{nm}$.

CD (MeOH): $\lambda_{\max }[\Theta]=214$ (22528), 219 (19781), 240 (-3914), 249 (-1481), 283 (-11875), 306 (5207), 313 (5414), 327 (2313) nm.

${ }^{1} \mathrm{H}-\mathrm{NMR}\left(600 \mathrm{MHz}, \mathrm{CD}_{2} \mathrm{Cl}_{2}\right): \delta_{\mathrm{H}}=1.45\left(\mathrm{~d}, J=6.5 \mathrm{~Hz}, 3 \mathrm{H}, 10-\mathrm{H}_{3}\right), 2.03(\mathrm{~m}, 1 \mathrm{H}$, $\left.3-\mathrm{H}_{\mathrm{A}}\right), 2.27\left(\mathrm{~m}, 1 \mathrm{H}, 3-\mathrm{H}_{\mathrm{B}}\right), 2.56\left(\mathrm{ddd}, J=18.0,10.5,5.0 \mathrm{~Hz}, 1 \mathrm{H}, 2-\mathrm{H}_{\mathrm{A}}\right), 2.80(\mathrm{~m}$, $\left.1 \mathrm{H}, 2-\mathrm{H}_{\mathrm{B}}\right), 3.88\left(\mathrm{~s}, 3 \mathrm{H}, 6-\mathrm{OCH}_{3}\right), 4.77$ (dd, $\left.J=9.0,4.0 \mathrm{~Hz}, 1 \mathrm{H}, 4-\mathrm{H}\right), 5.18$ (q, $J=6.5 \mathrm{~Hz}, 1 \mathrm{H}, 9-\mathrm{H}), 6.68(\mathrm{~s}, 1 \mathrm{H}, 5-\mathrm{H}), 13.19$ (s, 1H, 8-OH) ppm.

${ }^{13}$ C-NMR (150 MHz, $\mathrm{CD}_{2} \mathrm{Cl}_{2}$ ): $\delta_{\mathrm{C}}=22.96$ (q, C-10), 31.99 (t, C-3), 35.31 (t, C-2), 56.17 (q, $\mathrm{OCH}_{3}$ ), 63.56 (d, C-9), 67.97 (d, C-4), 101.06 (d, C-5), 110.27 (s, C-8a), 118.61 (s, C-7), 148.36 (s, C-4a), 161.40 (s, C-8), 162.89 (s, C-6), 203.34 (s, C-1) ppm.

Naphthalacton A (무)

$\mathrm{C}_{16} \mathrm{H}_{18} \mathrm{O}_{7}(322.31)$

$\mathbf{R}_{\mathbf{f}}$-Wert: 0.34 (Chloroform/Methanol 9:1)

0.32 (Cyclohexan/Essigester/Methanol 5:10:2)

0.33 (Essigester/Methanol 19:1).

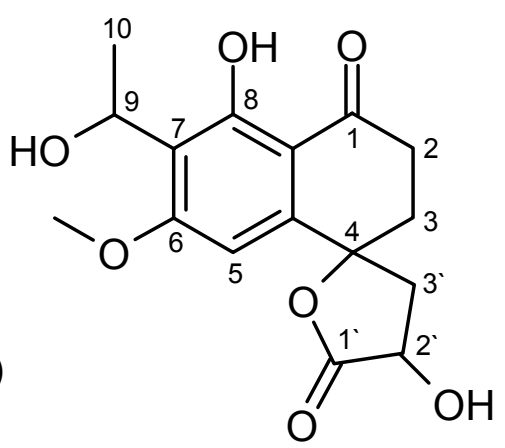

Anfärbeverhalten: blaugrau (Anis).

$[\alpha]_{D}^{20}=+14^{\circ}(\mathrm{c}=1.0 \mathrm{mg} / \mathrm{mL}$ in Methanol).

ESI-MS: $\mathrm{m} / \mathrm{z}=345.0[\mathrm{M}+\mathrm{Na}]^{+}, 666.9[2 \mathrm{M}+\mathrm{Na}]^{+}, 321.2[\mathrm{M}-\mathrm{H}]^{-}$.

El-MS: $\mathrm{m} / \mathrm{z}(\%)=322.1(6)[\mathrm{M}]^{+}, 307.1(100)\left[\mathrm{M}-\mathrm{CH}_{3}\right]^{+}, 231.1(17)$. 
IR $(\mathrm{KBr}): \tilde{v}=3430,2952,1780,1621$ 1486, 1411, 1342, 1263, 1134, 956, 838 $\mathrm{cm}^{-1}$.

UV $(\mathrm{MeOH}) \quad: \lambda_{\max }(\varepsilon)=209$ (2717), 256 (3794), 293 sh (615);

$(\mathrm{MeOH} / \mathrm{HCl}) \quad: \lambda_{\max }(\varepsilon)=209$ (2128), 256 (3610), 292 sh (545);

$(\mathrm{MeOH} / \mathrm{NaOH}): \lambda_{\max }(\varepsilon)=256$ (3340), 300 (364) nm.

CD $(\mathrm{MeOH}): \lambda_{\max }[\Theta]=222$ (-590), 249 (-7276), 252 (-2373), 284 (-9700), 308 (8019), 311 (7841), 316 (8008), 328 (3934) nm.

${ }^{1} \mathrm{H}-N M R\left(600 \mathrm{MHz}, \mathrm{CD}_{2} \mathrm{Cl}_{2}\right): \delta_{\mathrm{H}}=1.48\left(\mathrm{~d}, J=6.5 \mathrm{~Hz}, 3 \mathrm{H}, 10-\mathrm{H}_{3}\right), 2.21$ (ddd, $J=13.5,4.5,4.5 \mathrm{~Hz}, 1 \mathrm{H}, 3-\mathrm{H}_{\mathrm{A}}$ ), 2.24 (ddd, $J=13.5,10.0,1.0 \mathrm{~Hz}, 1 \mathrm{H}, 3^{\prime}-\mathrm{H}_{\mathrm{A}}$ ), 2.41 (dddd, $J=13.0,12.5,5.5,1.0 \mathrm{~Hz}, 1 \mathrm{H}, 3-\mathrm{H}_{\mathrm{B}}$ ), 2.79 (ddd, $J=18.0,12.0,5.0 \mathrm{~Hz}, 1 \mathrm{H}$, 2- $\mathrm{H}_{\mathrm{A}}$ ), $2.83\left(\mathrm{ddd}, J=18.0,5.5,4.0 \mathrm{~Hz}, 1 \mathrm{H}, 2-\mathrm{H}_{\mathrm{B}}\right), 2.99(\mathrm{dd}, J=13.5,8.5 \mathrm{~Hz}, 1 \mathrm{H}$, $\left.3^{\prime}-\mathrm{H}_{\mathrm{B}}\right), 3.91\left(\mathrm{~s}, 3 \mathrm{H}, 6-\mathrm{OCH}_{3}\right), 4.76\left(\mathrm{dd}, J=9.5,8.5 \mathrm{~Hz}, 1 \mathrm{H}, 2{ }^{\prime}-\mathrm{H}\right), 5.22$ (q, $J=6.5 \mathrm{~Hz}, 1 \mathrm{H}, 9-\mathrm{H}), 6.63(\mathrm{~s}, 1 \mathrm{H}, 5-\mathrm{H}), 13.25$ (s, 1H, 8-OH) ppm.

${ }^{13}$ C-NMR (150 MHz, $\mathrm{CD}_{2} \mathrm{Cl}_{2}$ ): $\delta_{\mathrm{C}}=22.85$ (q, C-10), 33.13 (t, C-3), $34.64(\mathrm{t}, \mathrm{C}-2)$, 41.14 (t, C-3'), 56.51 (q, OCH $\left.{ }_{3}\right), 63.55$ (d, C-9), 68.39 (d, C-2'), 81.69 (s, C-4), 99.70 (d, C-5), 110.35 (s, C-8a), 120.09 (s, C-7), 146.19 (s, C-4a), 161.65 (s, C-8), 163.57 (s, C-6), 176.40 (s, C-1'), 201.17 (s, C-1) ppm.

\section{Naphthalacton B $\underline{(\underline{61})}$}

$\mathrm{C}_{16} \mathrm{H}_{18} \mathrm{O}_{6}(306.31)$

$\mathbf{R}_{\mathbf{f}}$-Wert: 0.44 (Chloroform/Methanol 9:1)

0.47 (Cyclohexan/Essigester/Methanol 5:10:2) 0.45 (Essigester/Methanol 19:1).<smiles>CCc1c(OC)cc2c(c1O)C(=O)CCC21CC(O)C(=O)O1</smiles>

Anfärbeverhalten: braun (Anis).

$[\alpha]_{D}^{20}=+16^{\circ}(\mathrm{c}=1.0 \mathrm{mg} / \mathrm{mL}$ in Methanol).

ESI-MS: $\mathrm{m} / \mathrm{z}=307.3[\mathrm{M}+\mathrm{Na}]^{+}, 305.3[\mathrm{M}-\mathrm{H}]^{-}$. 
IR $(\mathrm{KBr}): \tilde{v}=3427,2942,2869,1775,1623,1575,1495,1456,1414,1361,1316$, $1260,1172,1136,1047,958,840 \mathrm{~cm}^{-1}$.

UV $(\mathrm{MeOH}) \quad: \lambda_{\max }(\varepsilon)=203$ (5813), 224 (8003), 237 sh (5220), 287 (5837), 323 sh (1519);

$(\mathrm{MeOH} / \mathrm{HCl}) \quad: \lambda_{\max }(\varepsilon)=224$ (7729), 239 sh (5024), 287 (5785), 326 sh (1432);

$(\mathrm{MeOH} / \mathrm{NaOH}): \lambda_{\max }(\varepsilon)=203$ (6714), 223 (8105), 237 sh (5454), 288 (5985), 327 $\mathrm{sh}(1955) \mathrm{nm}$.

CD $(\mathrm{MeOH}): \lambda_{\max }[\Theta]=220$ (5620), 240 (-5005), 251 (-264), 285 (-9076), 307 (9834), 310 (9689), 315 (9928) nm.

${ }^{1} \mathrm{H}-\mathrm{NMR}\left(600 \mathrm{MHz}, \mathrm{CDCl}_{3}\right): \delta_{\mathrm{H}}=1.05\left(\mathrm{t}, J=7.5,3 \mathrm{H}, 10-\mathrm{H}_{3}\right), 2.21$ (ddd, $J=13.5$, 4.5, 4.5 Hz, 1H, 3- $\mathrm{H}_{\mathrm{A}}$ ), 2.29 (dd, J = 13.5, $\left.10.0 \mathrm{~Hz}, 1 \mathrm{H}, 3^{\prime}-\mathrm{H}_{\mathrm{A}}\right), 2.42$ (td, J = 13.0, $5.0 \mathrm{~Hz}, 1 \mathrm{H}, 3-\mathrm{H}_{\mathrm{B}}$ ), 2.62 (q, J = 7.5 Hz, 2H, 9- $\mathrm{H}_{2}$ ), 2.74 (ddd, $J=18.0,12.5,5.0 \mathrm{~Hz}$, $1 \mathrm{H}, 2-\mathrm{H}_{\mathrm{A}}$ ), 2.84 (ddd, $J=18.0,4.5,4.5 \mathrm{~Hz}, 1 \mathrm{H}, 2-\mathrm{H}_{\mathrm{B}}$ ), 2.98 (dd, $J=13.5,8.5 \mathrm{~Hz}$, $\left.1 \mathrm{H}, 3^{\prime}-\mathrm{H}_{\mathrm{B}}\right), 3.87\left(\mathrm{~s}, 3 \mathrm{H}, 6-\mathrm{OCH}_{3}\right), 4.76\left(\mathrm{dd}, J=9.5,9.0 \mathrm{~Hz}, 1 \mathrm{H}, 2^{\prime}-\mathrm{H}\right), 6.56(\mathrm{~s}, 1 \mathrm{H}$, $5-\mathrm{H}), 12.81(\mathrm{~d}, \mathrm{~J}=1 \mathrm{~Hz}, 1 \mathrm{H}, 8-\mathrm{OH}) \mathrm{ppm}$.

${ }^{13}$ C-NMR (150 MHz, $\left.\mathrm{CDCl}_{3}\right): \delta_{\mathrm{C}}=12.93$ (q, C-10), $15.59(\mathrm{t}, \mathrm{C}-9), 33.00$ (t, C-3),

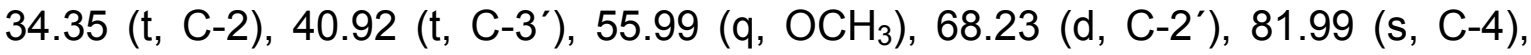
98.81 (d, C-5), 109.82 (s, C-8a), 120.05 (s, C-7), 143.87 (s, C-4a), 161.97 (s, C-8), 164.11 (s, C-6), 176.65 (s, C-1'), 199.96 (s, C-1) ppm.

\subsection{Acetylierung von 6-Methoxyparvulenon ( $\underline{56})$}

$12.1 \mathrm{mg}$ ( $0.048 \mathrm{mmol}) \underline{\mathbf{5 6}}$ wurden in $2 \mathrm{~mL}$ Dichlormethan gelöst und mit $1 \mathrm{~mL}$ Pyridin versetzt. Die Mischung wurde auf $0^{\circ} \mathrm{C}$ abgekühlt, anschließend $1 \mathrm{~mL}$ Acetanhydrid zugefügt und gerührt. Der Reaktionsverlauf wurde alle 10 min durch DCAnalyse des Reaktionsgemisches kontrolliert. Nach 2 Stunden beendete man die Reaktion durch Zugabe von $20 \mathrm{~mL}$ Eis/Wasser. Das Reaktionsgemisch wurde dreimal mit jeweils $20 \mathrm{~mL}$ Dichlormethan ausgeschüttelt, die organische Phase eingeengt und mit Toluol koevaporiert. Die dünnschichtchromatographische Analyse des Rohproduktes zeigte zwei Spots. Nach Reinigung an Sephadex LH-20 
(Aceton) konnten zwei Acetylierungsprodukte $\underline{\mathbf{5 5}}$ (6.0 mg, $0.018 \mathrm{mmol}, 37 \%)$ und $\underline{54}$ (2.7 mg, $0.009 \mathrm{mmol}, 19$ \%) gewonnen werden.

\section{4,9-Di-O-acetyl-6-methoxyparvulenon (ㅌ5)}

$\mathrm{C}_{17} \mathrm{H}_{20} \mathrm{O}_{7}(336.34)$

$\mathbf{R}_{\mathbf{f}}$-Wert: 0.70 (Chloroform/Methanol 9:1)

0.58 (Cyclohexan/Essigester/Methanol 5:10:2)

0.55 (Essigester/Methanol 19:1).<smiles>COc1cc2c(c(O)c1C(C)OC(C)=O)C(=O)CCC2OC(C)=O</smiles>

Anfärbeverhalten: blaugrau (Anis).

$[\alpha]_{D}^{20}=-67^{\circ}(\mathrm{c}=1.0 \mathrm{mg} / \mathrm{mL}$ in Methanol $)$.

ESI-MS: $\mathrm{m} / \mathrm{z}=358.9[\mathrm{M}+\mathrm{Na}]^{+}, 694.8[2 \mathrm{M}+\mathrm{Na}]^{+}$.

EI-MS: $\mathrm{m} / \mathrm{z}(\%)=336.2(3)[\mathrm{M}]^{+}, 293.2(4)\left[\mathrm{M}-\mathrm{COCH}_{3}\right]^{+}, 276.2(7)\left[\mathrm{M}-\mathrm{COCH}_{3}-\mathrm{OH}\right]^{+}$, $43.0(100)\left[\mathrm{COCH}_{3}\right]^{+}$.

IR (KBr): $\tilde{v}=3436,3241,2964,2934,2865,1735,1627,1498,1421,1375,1282$, $1238,1161,1122,1067,1028,951,836,558 \mathrm{~cm}^{-1}$.

UV $(\mathrm{MeOH}) \quad: \lambda_{\max }(\varepsilon)=224$ (15442), 239 sh (9148), 284 (12520), 319 sh (4251),

$(\mathrm{MeOH} / \mathrm{HCl}) \quad: \lambda_{\max }(\varepsilon)=224$ (13766), 238 sh (7534), 284 (11880), 318 sh (2449), 335 sh (2072),

$(\mathrm{MeOH} / \mathrm{NaOH}): \lambda_{\max }(\varepsilon)=203$ (16220), 223 (16403), 237 sh (10117), 285 (13540), $316 \mathrm{sh}(4843) \mathrm{nm}$.

CD (MeOH): $\lambda_{\max }[\Theta]=208$ (10085), 210 (9777), 212 (10317), 215 (9554), 220 (12416), 240 (-7451), 257 (-2228), 270 (-3310), 291 (-622), 309 (-5524), 313 (-4693), 321 (-6425), 329 (-4169), 332 (-4532) nm.

${ }^{1} \mathrm{H}-\mathrm{NMR}\left(600 \mathrm{MHz}, \mathrm{CD}_{2} \mathrm{Cl}_{2}\right): \delta_{\mathrm{H}}=1.56\left(\mathrm{~d}, J=6.5 \mathrm{~Hz}, 3 \mathrm{H}, 10-\mathrm{H}_{3}\right), 1.97(\mathrm{~s}, 3 \mathrm{H}$, 14- $\left.\mathrm{H}_{3}\right), 2.07\left(\mathrm{~s}, 3 \mathrm{H}, 12-\mathrm{H}_{3}\right), 2.22\left(\mathrm{~m}, 1 \mathrm{H}, 3-\mathrm{H}_{\mathrm{A}}\right), 2.29\left(\mathrm{~m}, 1 \mathrm{H}, 3-\mathrm{H}_{\mathrm{B}}\right), 2.61$ (ddd, 
$J=18.0,5.0,4.5 \mathrm{~Hz}, 1 \mathrm{H}, 2-\mathrm{H}_{\mathrm{A}}$ ), 2.90 (ddd, $J=18.0,10.0,5.0 \mathrm{~Hz}, 1 \mathrm{H}, 2-\mathrm{H}_{\mathrm{B}}$ ), 3.90 (s, 3H, 6- $\mathrm{OCH}_{3}$ ), 5.97 (dd, J = 5.5, 3.5 Hz, 1H, 4-H), 6.27 (q, J = 6.5 Hz, 1H, 9-H), $6.52(\mathrm{~s}, 1 \mathrm{H}, 5-\mathrm{H}), 13.14(\mathrm{~s}, 1 \mathrm{H}, 8-\mathrm{OH}) \mathrm{ppm}$.

${ }^{13}$ C-NMR (150 MHz, CD $\mathrm{Cl}_{2}$ ): $\delta_{\mathrm{C}}=18.46$ (q, C-14), 21.21 (q, C-10), 21.29 (q, C-12), 28.14 (t, C-3), 33.95 (t, C-2), 56.35 (q, OCH ) $_{3} 64.88$ (d, C-9), 69.33 (d, C-4), 103.07 (d, C-5), 110.99 (s, C-8a), 116.20 (s, C-7), 143.12 (s, C-4a), 162.84 (s, C-8), 163.85 (s, C-6), 170.46 (s, C-11), 170.55 (s, C-13), 203.14 (s, C-1) ppm.

\section{4-O-Acetyl-6-methoxyparvulenon (54)}

$\mathrm{C}_{15} \mathrm{H}_{18} \mathrm{O}_{6}(294.30)$

$\mathbf{R}_{\mathbf{f}}$-Wert: 0.66 (Chloroform/Methanol 9:1)

0.49 (Cyclohexan/Essigester/Methanol 5:10:2)

0.46 (Essigester/Methanol 19:1).<smiles>COc1cc2c(c(O)c1C(C)O)C(=O)CCC2OC(C)=O</smiles>

Anfärbeverhalten: blaugrau (Anis).

$[\alpha]_{D}^{20}=-56^{\circ}(\mathrm{c}=1 \mathrm{mg} / \mathrm{mL}$ in Methanol).

ESI-MS: $\mathrm{m} / \mathrm{z}=317.0[\mathrm{M}+\mathrm{Na}]^{+}, 610.9[2 \mathrm{M}+\mathrm{Na}]^{+}, 293.2[\mathrm{M}-\mathrm{H}]^{-}$.

El-MS: $m / z(\%)=294.1(8)[M]^{+}, 279.1(65)\left[M-\mathrm{CH}_{3}\right]^{+}, 219.1$ (100), 43.0 (100) $\left[\mathrm{COCH}_{3}\right]^{+}$.

IR $(\mathrm{KBr}): \tilde{v}=3563,3441,2961,2926,2858,1736,1626,1575,1498,1461,1416$, $1371,1279,1228,1148,1111,1083,1064,1026,943,830 \mathrm{~cm}^{-1}$.

UV $(\mathrm{MeOH}) \quad: \lambda_{\max }(\varepsilon)=201$ (7681), 223 (13868), 237 sh (8272), 284 (11527), 317 sh (3768),

$(\mathrm{MeOH} / \mathrm{HCl}) \quad: \lambda_{\max }(\varepsilon)=223$ (12889), 239 sh (7045), 284 (11275), 317 sh (2430), 335 sh (2129),

$(\mathrm{MeOH} / \mathrm{NaOH}): \lambda_{\max }(\varepsilon)=201$ (14369), 204 (15667), 225 (13520), 235 sh (11727), 284 (11279), 327 (3708) nm. 
CD (MeOH): $\lambda_{\max }[\Theta]=207$ (10278), 209 (9656), 211 (9911), 213 (9517), 221 (13069), 240 (-2405), 250 (470), 277 (-2547), 292 (-849), 309 (-3830), 314 (-3579), $321(-4307) \mathrm{nm}$.

${ }^{1} \mathrm{H}-\mathrm{NMR}\left(600 \mathrm{MHz}, \mathrm{CD}_{2} \mathrm{Cl}_{2}\right): \delta_{\mathrm{H}}=1.47\left(\mathrm{~d}, J=6.5 \mathrm{~Hz}, 3 \mathrm{H}, 10-\mathrm{H}_{3}\right), 2.07(\mathrm{~s}, 3 \mathrm{H}$, 12- $\left.\mathrm{H}_{3}\right), 2.22\left(\mathrm{~m}, 1 \mathrm{H}, 3-\mathrm{H}_{\mathrm{A}}\right), 2.30\left(\mathrm{~m}, 1 \mathrm{H}, 3-\mathrm{H}_{\mathrm{B}}\right), 2.63$ (ddd, J = 18.0, 5.0, $4.5 \mathrm{~Hz}$, $1 \mathrm{H}, 2-\mathrm{H}_{\mathrm{A}}$ ), 2.91 (ddd, $\left.J=18.0,10.0,5.0 \mathrm{~Hz}, 1 \mathrm{H}, 2-\mathrm{H}_{\mathrm{B}}\right), 3.89\left(\mathrm{~s}, 3 \mathrm{H}, 6-\mathrm{OCH}_{3}\right), 5.20$ (q, $J=6.5 \mathrm{~Hz}, 1 \mathrm{H}, 9-\mathrm{H}), 5.98(\mathrm{dd}, J=5.5,3.5 \mathrm{~Hz}, 1 \mathrm{H}, 4-\mathrm{H}), 6.55(\mathrm{~s}, 1 \mathrm{H}, 5-\mathrm{H})$, $13.20(\mathrm{~s}, 1 \mathrm{H}, 8-\mathrm{OH}) \mathrm{ppm}$.

${ }^{13}$ C-NMR (150 MHz, $\mathrm{CD}_{2} \mathrm{Cl}_{2}$ ): $\delta_{\mathrm{C}}=21.29$ (q, C-12), 23.02 (q, C-10), 28.23 (t, C-3), 33.95 (t, C-2), 56.28 (q, OCH $), 63.55$ (d, C-9), 69.25 (d, C-4), 103.21 (d, C-5), 111.13 (s, C-8a), 120.26 (s, C-7), 142.29 (s, C-4a), 161.71 (s, C-8), 162.69 (s, C-6), 170.46 (s, C-11), 203.14 (s, C-1) ppm.

\subsection{Wachstumskurve}

Zur Aufnahme des zeitabhängigen Produktionsverlaufes von WDMH 28 wurde der Stamm in 1 L-Schüttelkolben ohne Schikanen und $250 \mathrm{~mL}$ Nährlösung 1158 bei $28^{\circ} \mathrm{C}$ und $120 \mathrm{spm}$ kultiviert. Ab der 42. Stunde wurden alle $12 \mathrm{~h}$ zwei Kolben entnommen und diese nach dem beschriebenen Schema (Abbildung 4) aufgearbeitet. Die erhaltenen Extrakte wurden dünnschichtchromatographisch und mit Hilfe der HPLC 1 (Säule 2, Programm 2) analysiert.

\subsection{Biogeneseuntersuchungen}

Die Fütterungsexperimente (1 L-Ansatz) wurden in jeweils vier $1 \mathrm{~L}-K o l b e n$ ohne Schikanen mit $250 \mathrm{~mL}$ Nährlösung 1158 durchgeführt. Nach dem Animpfen mit je einem $3 \mathrm{~cm}^{2}$ großem Stück einer gut bewachsenen Agarplatte wurde der Stamm bei $28^{\circ} \mathrm{C}$ und $120 \mathrm{spm}$ inkubiert. Die zu fütternde Substanz wurde in bidestilliertem Wasser gelöst, die Lösung auf einen pH-Wert von 6.5 eingestellt und autoklaviert. Ab der 66. Stunde wurden mittels einer Pumpe pro Kolben kontinuierlich $50 \mathrm{~mL}$ Lösung über 36 Stunden zugefüttert. Nach einer Kultivierungsdauer von 4 Tagen und 18 Stunden (12 Stunden Nachlauf) wurde der Ansatz geerntet und nach den allgemeinen Bedingungen (Abbildung 4) aufgearbeitet. Als Referenz ließ man $1 \mathrm{~L}$ Kultur ohne Zufütterung mitlaufen. Sowohl der Kultur- 
filtrat-Extrakt als auch der Myzel-Extrakt wurden an Kieselgel $\left(\mathrm{CH}_{2} \mathrm{Cl}_{2} / \mathrm{MeOH} 12: 1\right)$ säulenchromatographiert. Bei der weiteren Aufreinigung beschränkte man sich auf die Fraktion mit den Substanzen $\underline{\mathbf{5 6}}$ und $\underline{\mathbf{6 0}}$, die unter Verwendung einer Sephadex LH-20-Säule (Aceton) getrennt wurden.

\begin{tabular}{|c|c|c|c|c|}
\hline markierte Substanz & Firma & $\begin{array}{c}{[\%]} \\
\text { Anreicherung }\end{array}$ & {$[\mathrm{mg} / \mathrm{L}]$} & {$[\mathrm{mmol} / \mathrm{L}]$} \\
\hline $\mathrm{Na}-\left[1-{ }^{13} \mathrm{C}\right]$ Acetat & Campro Scientific & 99 & 750 & 9.1 \\
\hline $\mathrm{Na}-\left[1,2-{ }^{13} \mathrm{C}_{2}\right]$ Acetat & $\begin{array}{c}\text { Cambridge Isotope } \\
\text { Laboratories }\end{array}$ & 99 & 500 & 6.1 \\
\hline $\mathrm{Na}-\left[1,4-{ }^{13} \mathrm{C}_{2}\right]$ Succinat & $\begin{array}{c}\text { Cambridge Isotope } \\
\text { Laboratories }\end{array}$ & 99 & 135 & 2.2 \\
\hline$\left[2-{ }^{13} \mathrm{C}\right]$ Malonsäure & Isotec & 99 & 919 & 8.3 \\
\hline
\end{tabular}

Tabelle 15: Zum Stamm WDMH28 zugefütterte markierte Verbindungen.

\section{Stamm WDMH 46}

\subsection{Kultivierung}

Der Stamm WDMH 46 wurde in $1 \mathrm{~L}$-Erlenmeyerkolben ohne Schikanen, befüllt mit jeweils $170 \mathrm{~mL}$ Nährlösung 1405, beimpft mit je einem $3 \mathrm{~cm}^{2}$ großem Stück einer gut bewachsenen Agarplatte, bei $28^{\circ} \mathrm{C}$ und $120 \mathrm{spm}$ fermentiert. Nach 6- bzw. 14tägiger Kultivierung wurden die Kolben geerntet.

\subsection{Variation der Kultivierungsbedingungen}

\subsubsection{Kultivierung in Schüttelkolben mit XAD-Zusatz}

$\mathrm{Zu}$ den wie oben bereiteten Erlenmeyerkolben gab man jeweils $3 \mathrm{~g}$ XAD-2. Mit dem Zusatz in der Nährlösung wurden die Kolben autoklaviert, anschließend mit WDMH 46 beimpft und 6 Tage inkubiert.

\subsubsection{Kultivierung in Schüttelkolben mit Salz-Zusatz}

Die Kultivierung erfolgte analog zu 8.1. Zu den Nährmedien wurde zusätzlich $\mathrm{NaCl}$ zugesetzt, bis Konzentrationen von 0.2, 1, 2 und 4\% Salzgehalt erreicht wurden. 


\subsubsection{Kultivierung in Schüttelkolben als Ruhekultur mit und ohne Salz}

Die Ruhefermentation in $1 \mathrm{~L}$-Erlenmeyerkolben erfolgte in sieben Nährmedien. Je $500 \mathrm{~mL}$ NL 1158, E2, G20, 1405, 1549, Marine Broth und Biomalz pro Kolben wurden beimpft und 14 Tage bei $28^{\circ} \mathrm{C}$ inkubiert. Parallel wurde eine Versuchsreihe erstellt, bei der die Nährlösungen mit $\mathrm{NaCl}$ auf einen Salzgehalt von $3 \%$ gebracht wurden.

\subsubsection{Kultivierung in P-Kolben}

Für die Kultivierung in P-Kolben wurden 1L des Nährmediums (NL 1158, E2 und 1405) mit ca. $5 \mathrm{~cm}^{2}$ eines Pilzrasens von Agarplatte (NL E2) beimpft und anschließend bei $28^{\circ} \mathrm{C}$ für 24 Tage bebrütet. Die Kultivierung erfolgte als Ruhekultur.

\subsubsection{Kultivierung in P-Kolben als Festkultur}

Die Kultivierung in P-Kolben auf fester Phase wurde analog zu den Ruhefermentationen in Schüttelkolben in sieben Nährmedien ohne und mit 3\% $\mathrm{NaCl}$-Gehalt durchgeführt. $500 \mathrm{~mL}$ der einzelnen Nährmedien, abgefüllt in kleinen P-Kolben, wurde mit $11 \mathrm{~g}$ Agar versetzt, autoklaviert und zum Festwerden stehen gelassen. Anschließend wurde der feste Nährboden mit $5 \mathrm{~mL}$ einer 4 Tage alten Flüssigkultur (NL $1158,28^{\circ} \mathrm{C}, 120 \mathrm{spm}$ ) beimpft und 14 Tage bei $28^{\circ} \mathrm{C}$ und Dunkelheit inkubiert.

\subsubsection{Kultivierung in Fermentern}

\section{L-Maßstab:}

Die Kultivierung des Stammes WDMH 46 in einem 2 L-Biostat-B-Fermenter wurde mit dem Nährmedium 1158 durchgeführt. Das Animpfen erfolgte mit 3 Tage alten Vorkulturen (10\% des Fermentationsvolumens), die in $1 \mathrm{~L}$ Schüttelkolben (170 mL $\mathrm{NL} 1158$ ) bei $28^{\circ} \mathrm{C}$ und $120 \mathrm{spm}$ angezogen wurden. Die Kultivierung im $2 \mathrm{~L}$ Maßstab erfolgte bei $28^{\circ} \mathrm{C}$, einer Luftzufuhr von $1.0 \mathrm{vvm}$ und einer Rührerdrehzahl von 500 rpm. Nach 9 Stunden wurde die Luftzufuhr auf 2.0 vvm erhöht. Die Ernte erfolgte nach sieben Tagen.

5 L-Maßstab:

Die Kultivierung des Stammes WDMH 46 in einem 5 L-Biostat-B-Fermenter wurde im Nährmedium 1158 durchgeführt. Das Animpfen erfolgte mit 3 Tage alten Vor- 
kulturen (10\% des Fermentationsvolumens), die in $1 \mathrm{~L}$ Schüttelkolben (170 mL NL 1158 ) bei $28^{\circ} \mathrm{C}$ und $120 \mathrm{spm}$ angezogen wurden. Die Kultivierung im 5 L-Maßstab erfolgte bei $28^{\circ} \mathrm{C}$, einer Luftzufuhr von $1.0 \mathrm{vvm}$ und einer Rührerdrehzahl von 500 rpm. Die Ernte erfolgte nach sieben Tagen.

\section{L-Maßstab:}

Für die Kultivierung in einem 10 L-Biostat-E-Fermenter wurde die Nährlösung 1158 benutzt. Das Animpfen erfolgte mit 3 Tage alten Vorkulturen (10\% des Fermentationsvolumens), die in $1 \mathrm{~L}$ Schüttelkolben (170 mL NL 1158) bei $28^{\circ} \mathrm{C}$ und $120 \mathrm{spm}$ angezogen wurden. Die Kultivierung erfolgte bei $28^{\circ} \mathrm{C}$, einer $\mathrm{pH}$ Sperre von $5.5+/-2.5$, einer Luftzufuhr von 1.0 vvm und einer Rührerdrehzahl von 300 rpm. Die Ernte erfolgte nach sechs Tagen.

\section{L-Airlifter:}

Der Airlifter wurde mit $9.5 \mathrm{~L}$ Nährmedium 1405 und $4 \mathrm{~g}$ Entschäumer befüllt, autoklaviert und mit 4 Tage alten Vorkulturen beimpft $(8 \%$ des Fermentationsvolumens). Diese wurden in 1 L-Schüttelkolben (170 mL NL 1158/1405) bei $28^{\circ} \mathrm{C}$ und $120 \mathrm{spm}$ angezogen. Die Kultivierung im Airlift-Fermenter erfolgte bei $28^{\circ} \mathrm{C}$ mit einer Belüftung von $4.0 \mathrm{vvm}$. Die Fermentation wurde durch Probenentnahme überwacht und nach fünf Tagen beendet.

10 L-Airlifter mit Überdruck:

Die Fermentation im 10 L-Airlift-Fermenter, befüllt mit 9.2 L 1405-Nährmedium und angeimpft mit $800 \mathrm{~mL}$ Vorkultur, erfolgte bei $28^{\circ} \mathrm{C}, 5$ bar Überdruck und einer Belüftung von 4 vvm.

\subsection{Aufarbeitung}

Bei allen Fermentationsansätzen erfolgte nach der Ernte eine Trennung in Myzel und Kulturfiltrat (Zentrifugation oder Filtration). Das Kulturfiltrat wurde $3 \times$ mit der Hälfte des Volumens an Essigester ausgeschüttelt und nach Entfernung des Lösungsmittels erhielt man den Kulturfiltrat-Extrakt. Das Myzel wurde mit Aceton versetzt und im Ultraschallbad extrahiert. Evaporierung des Lösungsmittels gab den Myzel-Extrakt.

\subsection{Isolierung und Charakterisierung der Metabolite}

Die Isolierung der Hauptmetabolite ist in nachfolgender Abbildung dargestellt. 


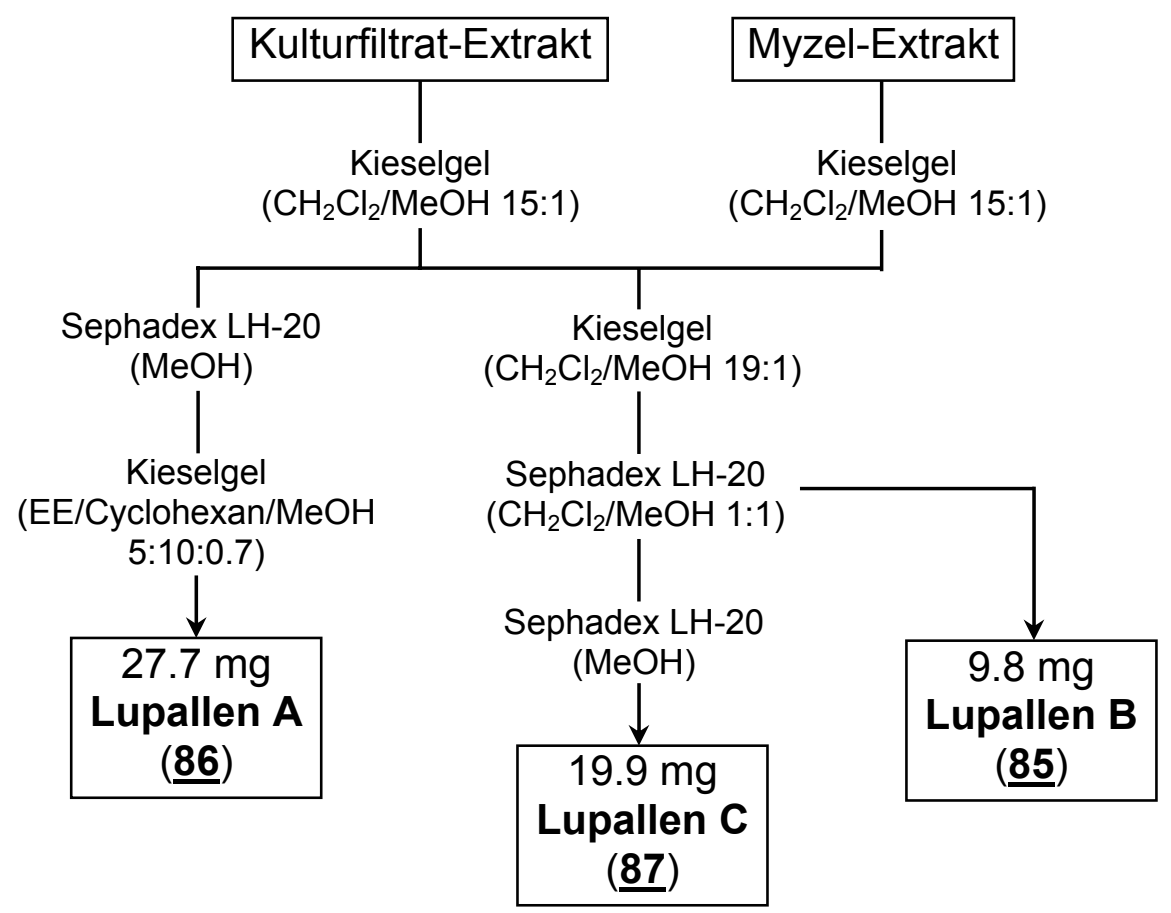

Abbildung 34: Isolierung der Hauptmetabolite aus WDMH46

Beim Zusatz von Acetat zum Nährmedium 1405 konnte aus dem Kulturfiltrat die Substanz Lupallen D (무) $(7.9 \mathrm{mg})$ durch Säulenchromatographie an Kieselgel $\left(\mathrm{CH}_{2} \mathrm{Cl}_{2} / \mathrm{MeOH}\right.$ 15:1) und Sephadex LH-20 (Aceton) erhalten werden. Diese erscheint auf dem DC einheitlich, zeigte aber in der HPLC-MS-Analyse zwei Substanzpeaks mit der gleichen Masse, die sich nicht weiter auftrennen ließen. Die Stereoisomere lagen im Verhältnis 3:2 vor und konnten durch NMRSpektroskopie zugeordnet werden.

Das Kulturfiltat aus dem 10 L-Airlift-Fermenter wurde an Kieselgel $\left(\mathrm{CH}_{2} \mathrm{Cl}_{2} / \mathrm{MeOH}\right.$ 15:1) säulenchromatographiert. Weitere Aufreinigung der 5. Rohprodukt-Fraktion an Sephadex LH-20 (MeOH) lieferte $195.8 \mathrm{mg}$ Lupallen E (191) als weißen Feststoff. Die 2. und 3. Fraktion der Rohprodukt-Säule wurden an Sephadex LH-20 (Aceton) weiter aufgereinigt. Man erhielt $12.7 \mathrm{mg}$ Lupaketon A (4) und $36.8 \mathrm{mg}$ 3-Dimethylallyl-4-hydroxybenzoesäure (흐) in Form eines farblosen Öles. Da der Stamm eine Vielzahl an Derivaten der bisher gefundenen Metabolite produziert, die einen $\mathrm{R}_{\mathrm{f}}$-Bereich von 0.2 bis 0.4 aufweisen und mit Anis pink anfärben, wurde dazu übergegangen, die einzelnen Fraktionen über die HPLC zu 
analysieren. So konnten den bislang aufgereinigten Sekundärmetabolite folgende Retentionszeiten zugeordnet werden (HPLC 1, Säule 2, Programm 3):

\begin{tabular}{cc}
\hline Substanz & Retentionszeit \\
\hline Lupallen A $(\underline{\mathbf{8 6}})$ & 17.9 \\
Lupallen B $(\underline{\mathbf{8 5}})$ & 18.9 \\
Lupallen C $(\underline{\mathbf{8 7}})$ & 17.6 \\
Lupallen D $(\underline{\mathbf{9 0}})$ & 29.3 \\
Lupallen E $(\underline{\mathbf{9 1}})$ & 9.3 \\
Lupaketon A $\underline{\mathbf{9 4}})$ & 10.9 \\
3-Dimethylallyl-4-OH-benzoesäure $(\underline{\mathbf{9 6}})$ & 29.2 \\
\hline
\end{tabular}

Tabelle 16: Retentionszeiten der isolierten Metabolite aus WDMH46 (HPLC 1, Säule 2, Programm 3).

Die Fraktionen, die in den HPLC-Chromatogrammen (HPLC 1, Säule 2, Programm 3) Peaks einer Retentionszeit von 29.4 min enthielten, wurden vereinigt und über Sephadex LH-20 (Aceton) aufgereinigt. 4-Hydroxy-3-(3'-hydroxy-3'methylbut-1-enyl)-benzoesäure (구) konnte mit einer Ausbeute von $19.5 \mathrm{mg}$ als farbloses Öl erhalten werden.

Des Weiteren gab es einige HPLC-Fraktionen, die Peaks mit einer Retentionszeit von 22.1 min enthielten (HPLC 1, Säule 2, Programm 3). Auch diese wurden vereinigt und über die präparative HPLC getrennt (HPLC 3, Säule 5, Programm 6). Dieser Auftrennungsschritt gab drei Reinsubstanzen als farbloses Öl: $1.3 \mathrm{mg}$

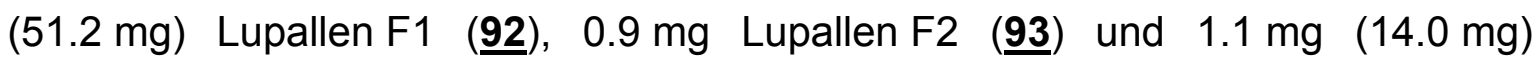
Lupaketon B (모). 


\section{Lupallen A $(\underline{86})$}

$\mathrm{C}_{16} \mathrm{H}_{24} \mathrm{O}_{4}(280.36)$

$\mathbf{R}_{\mathbf{f}}$-Wert: 0.28 (Chloroform/Methanol 9:1)

0.43 (Cyclohexan/Essigester/Methanol 5:10:2)

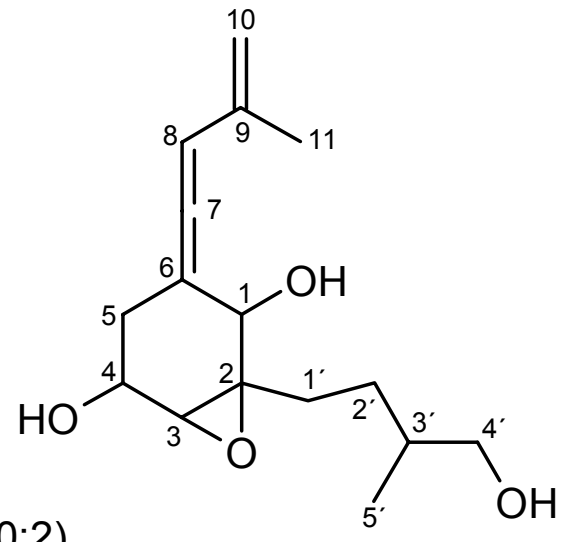

0.40 (Essigester/Methanol 19:1).

Anfärbeverhalten: pink (Anis), blau (Ehrlich), gelb-braun (Orcin).

$[\alpha]_{D}^{20}=+74^{\circ}(\mathrm{c}=1.0 \mathrm{mg} / \mathrm{mL}$ in Methanol $)$.

ESI-MS: $\mathrm{m} / \mathrm{z}=303.6[\mathrm{M}+\mathrm{Na}]^{+}, 583.6[2 \mathrm{M}+\mathrm{Na}]^{+}, 279.6[\mathrm{M}-\mathrm{H}]^{-}, 559.7[2 \mathrm{M}-\mathrm{H}]^{-}$.

HR-ESI-MS: 281.1747 gefunden wie berechnet für $\mathrm{C}_{16} \mathrm{H}_{25} \mathrm{O}_{4}$, 303.1566 gefunden wie berechnet für $\mathrm{C}_{16} \mathrm{H}_{24} \mathrm{O}_{4} \mathrm{Na}$.

IR $(\mathrm{KBr}): \tilde{v}=3388,2958,2922,2874,1955,1633,1455,1432,1385,1259,1036$, $886,560 \mathrm{~cm}^{-1}$.

UV $(\mathrm{MeOH}) \quad: \lambda_{\max }(\varepsilon)=220$ (20583), 231 sh (14447), 275 (888);

$(\mathrm{MeOH} / \mathrm{HCl}) \quad: \lambda_{\max }(\varepsilon)=220$ (19579), 232 sh (13000), 277 (569);

$(\mathrm{MeOH} / \mathrm{NaOH}): \lambda_{\max }(\varepsilon)=220$ (19012), 232 sh (12711), 275 (369) nm.

CD $(\mathrm{MeOH}): \lambda_{\max }[\Theta]=202$ (8577), 212 (21009), 216 (18041), 219 (19943), 232 (1301), 250 (14569) nm.

${ }^{1} \mathrm{H}-\mathrm{NMR}\left(600 \mathrm{MHz}, \mathrm{D}_{5}\right.$-Pyridin, $\left.35^{\circ} \mathrm{C}\right): \delta_{\mathrm{H}}=1.04\left(\mathrm{~d}, \mathrm{~J}=6.5 \mathrm{~Hz}, 3 \mathrm{H}, 5^{\prime}-\mathrm{H}_{3}\right), 1.55$ (dddd, $\left.J=12.5,12.0,8.0,5.0 \mathrm{~Hz}, 1 \mathrm{H}, 2^{\prime}-\mathrm{H}_{\mathrm{A}}\right), 1.81\left(\mathrm{~m}, 1 \mathrm{H}, 3^{\prime}-\mathrm{H}\right), 1.83(\mathrm{~s}, 3 \mathrm{H}$, $\left.11-\mathrm{H}_{3}\right), 1.91\left(\mathrm{~m}, 1 \mathrm{H}, 1^{\prime}-\mathrm{H}_{\mathrm{A}}\right), 2.08\left(\mathrm{~m}, 1 \mathrm{H}, 2^{\prime}-\mathrm{H}_{\mathrm{B}}\right), 2.51$ (ddd, $J=14.0,12.0,4.5 \mathrm{~Hz}$, $\left.1 \mathrm{H}, 1^{\prime}-\mathrm{H}_{\mathrm{B}}\right), 2.87\left(\mathrm{dd}, J=12.5,6.5 \mathrm{~Hz}, 1 \mathrm{H}, 5-\mathrm{H}_{\mathrm{A}}\right), 3.09$ (ddd, $J=12.5,8.5,3.0 \mathrm{~Hz}$, $\left.1 \mathrm{H}, 5-\mathrm{H}_{\mathrm{B}}\right), 3.63(\mathrm{brs}, 1 \mathrm{H}, 3-\mathrm{H}), 3.66\left(\mathrm{dd}, J=10.0,6.5 \mathrm{~Hz}, 1 \mathrm{H}, 4^{\prime}-\mathrm{H}_{\mathrm{A}}\right), 3.73(\mathrm{dd}$, $\left.J=10.5,6.0 \mathrm{~Hz}, 1 \mathrm{H}, 4^{\prime}-\mathrm{H}_{\mathrm{B}}\right), 4.59(\mathrm{t}, J=7.5 \mathrm{~Hz}, 1 \mathrm{H}, 4-\mathrm{H}), 4.85\left(\mathrm{~m}, 1 \mathrm{H}, 10-\mathrm{H}_{\mathrm{A}}\right)$, 
5.00 (brs, 1H, 1-H), $5.01\left(\mathrm{~m}, 1 \mathrm{H}, 10-\mathrm{H}_{\mathrm{B}}\right), 5.84$ (brs, 1H, 4'-OH), 6.15 (d, J = $3.0 \mathrm{~Hz}$, $1 \mathrm{H}, 8-\mathrm{H}), 7.15$ (brs, 1H, 1-OH), 7.28 (brs, 1H, 3-OH) ppm.

${ }^{13}$ C-NMR (150 MHz, D $\left.5^{-P y r i d i n, ~ 35 ㅇ) ~}\right): \delta_{\mathrm{C}}=17.19$ (q, C-5'), 19.71 (q, C-11), 27.76 (t, C-2'), 30.45 (t, C-1'), 32.12 (t, C-5), 36.68 (d, C-3'), 63.64 (d, C-3), 64.151 (s, C-2), 66.31 (d, C-4), 67.27 (t, C-4'), 70.27 (d, C-1), 97.94 (d, C-8), 103.21 (s, C-6), 113.64 (t, C-10), 140.04 (s, C-9), 204.03 (s, C-7) ppm.

${ }^{1} \mathrm{H}-\mathrm{NMR}\left(500 \mathrm{MHz}, \mathrm{CD}_{3} \mathrm{OD}\right): \delta_{\mathrm{H}}=0.91\left(\mathrm{~d}, \mathrm{~J}=6.5,3 \mathrm{H}, 5^{\prime}-\mathrm{H}_{3}\right), 1.18\left(\mathrm{~m}, 1 \mathrm{H}, 2^{\prime}-\mathrm{H}_{\mathrm{A}}\right)$, $1.51\left(\mathrm{~m}, 1 \mathrm{H}, 1^{\prime}-\mathrm{H}_{\mathrm{A}}\right), 1.55\left(\mathrm{~m}, 1 \mathrm{H}, 3^{\prime}-\mathrm{H}\right), 1.61\left(\mathrm{~m}, 1 \mathrm{H}, 2^{\prime}-\mathrm{H}_{\mathrm{B}}\right), 1.77\left(\mathrm{~s}, 3 \mathrm{H}, 11-\mathrm{H}_{3}\right)$, 2.07 (ddd, $\left.J=13.5,11.5,4.5 \mathrm{~Hz}, 1 \mathrm{H}, 1^{\prime}-\mathrm{H}_{\mathrm{B}}\right), 2.33\left(\mathrm{~m}, 1 \mathrm{H}, 5-\mathrm{H}_{\mathrm{A}}\right), 2.35(\mathrm{~m}, 1 \mathrm{H}$, 5- $\mathrm{H}_{\mathrm{B}}$ ), $3.03(\mathrm{~d}, J=1 \mathrm{~Hz}, 1 \mathrm{H}, 3-\mathrm{H}), 3.34\left(\mathrm{dd}, J=10.5,6.5 \mathrm{~Hz}, 1 \mathrm{H}, 4^{\prime}-\mathrm{H}_{\mathrm{A}}\right), 3.42$ (dd, $\left.J=10.5,5.5 \mathrm{~Hz}, 1 \mathrm{H}, 4^{\prime}-\mathrm{H}_{\mathrm{B}}\right), 3.92$ (dd, $\left.J=9.0,7.0 \mathrm{~Hz}, 1 \mathrm{H}, 4-\mathrm{H}\right), 4.34(\mathrm{~s}, 1 \mathrm{H}, 1-\mathrm{H})$, $4.81\left(\mathrm{~m}, 1 \mathrm{H}, 10-\mathrm{H}_{\mathrm{A}}\right), 4.90\left(\mathrm{~m}, 1 \mathrm{H}, 10-\mathrm{H}_{\mathrm{B}}\right), 5.93(\mathrm{~d}, J=3.5 \mathrm{~Hz}, 1 \mathrm{H}, 8-\mathrm{H}) \mathrm{ppm}$.

${ }^{13}$ C-NMR (125 MHz, CD ${ }_{3} \mathrm{OD}$ ): $\delta_{\mathrm{C}}=17.10$ (q, C-5'), 19.91 (q, C-11), 27.85 (t, C-2'), 30.61 (t, C-1'), 31.66 (t, C-5), 36.93 (d, C-3'), 63.95 (d, C-3), 64.60 (s, C-2), 66.85 (d, C-4), 68.03 (t, C-4'), 70.68 (d, C-1), 98.72 (d, C-8), 101.97 (s, C-6), 114.24 (t, C-10), 140.61 (s, C-9), 205.04 (s, C-7) ppm.

\section{Lupallen B (모)}

$\mathrm{C}_{16} \mathrm{H}_{24} \mathrm{O}_{4}(280.36)$

$\mathbf{R}_{\mathbf{f}}$-Wert: 0.27 (Chloroform/Methanol 9:1)

0.42 (Cyclohexan/Essigester/Methanol 5:10:2)

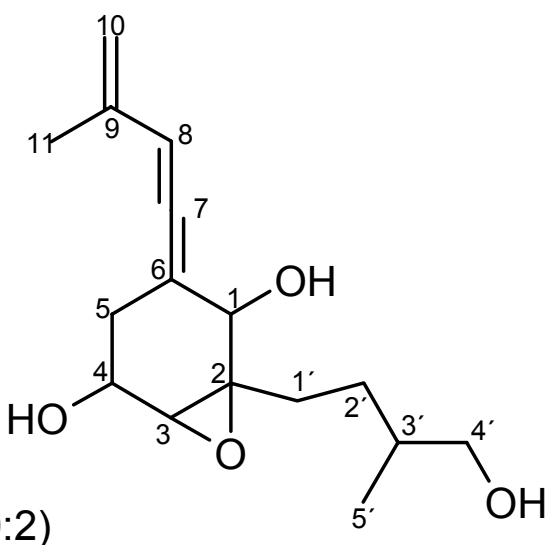

0.39 (Essigester/Methanol 19:1).

Anfärbeverhalten: pink (Anis), blau (Ehrlich), gelb-braun (Orcin).

$[\alpha]_{D}^{20}=+77^{\circ}(\mathrm{c}=1.0 \mathrm{mg} / \mathrm{mL}$ in Methanol $)$. 
ESI-MS: $\mathrm{m} / \mathrm{z}=303.4[\mathrm{M}+\mathrm{Na}]^{+}, 279.6[\mathrm{M}-\mathrm{H}]^{-}, 559.4[2 \mathrm{M}-\mathrm{H}]^{-}$.

HR-ESI-MS: 281.1747 gefunden wie berechnet für $\mathrm{C}_{16} \mathrm{H}_{25} \mathrm{O}_{4}$, 303.1566 gefunden wie berechnet für $\mathrm{C}_{16} \mathrm{H}_{24} \mathrm{O}_{4} \mathrm{Na}$.

IR $(\mathrm{KBr}): \tilde{v}=3388,2956,2922,2879,1957,1634,1455,1382,1260,1036,887$ $\mathrm{cm}^{-1}$.

UV $(\mathrm{MeOH}) \quad: \lambda_{\max }(\varepsilon)=209$ sh (16058), 219 (17610), 280 (2223);

$(\mathrm{MeOH} / \mathrm{HCl}) \quad: \lambda_{\max }(\varepsilon)=207$ sh (15179), 219 (16589), 276 (2544);

$(\mathrm{MeOH} / \mathrm{NaOH}): \lambda_{\max }(\varepsilon)=220(14763), 283(1144) \mathrm{nm}$.

CD $(\mathrm{MeOH}): \lambda_{\max }[\Theta]=202$ (4589), 204 (2210), 212 (4676), 215 (4346), 217 (5411), 231 (2339), 245 (7483) nm.

${ }^{1} \mathrm{H}-\mathrm{NMR}\left(600 \mathrm{MHz}, \mathrm{D}_{5}-\mathrm{Py}\right.$ ridin, $\left.35^{\circ} \mathrm{C}\right): \delta_{\mathrm{H}}=1.04$ (d, $\left.\mathrm{J}=7.0,3 \mathrm{H}, 5^{\prime}-\mathrm{H}_{3}\right), 1.53$ (dddd, $\left.J=12.5,12.0,8.0,5.0 \mathrm{~Hz}, 1 \mathrm{H}, 2^{\prime}-\mathrm{H}_{\mathrm{A}}\right), 1.85\left(\mathrm{~m}, 1 \mathrm{H}, 3^{\prime}-\mathrm{H}\right), 1.94\left(\mathrm{~m}, 1 \mathrm{H}, 1^{\prime}-\mathrm{H}_{\mathrm{A}}\right)$, $1.95\left(\mathrm{~s}, 3 \mathrm{H}, 11-\mathrm{H}_{3}\right), 2.08$ (tdd, $J=12.5,5.0,4.5 \mathrm{~Hz}, 1 \mathrm{H}, 2{ }^{\prime}-\mathrm{H}_{\mathrm{B}}$ ), 2.51 (ddd, $\left.J=14.0,12.0,4.5 \mathrm{~Hz}, 1 \mathrm{H}, 1^{\prime}-\mathrm{H}_{\mathrm{B}}\right), 2.82\left(\mathrm{dd}, J=12.5,6.5 \mathrm{~Hz}, 1 \mathrm{H}, 5-\mathrm{H}_{\mathrm{A}}\right), 3.08$ (ddd, $\left.J=12.5,9.5,3.5 \mathrm{~Hz}, 1 \mathrm{H}, 5-\mathrm{H}_{\mathrm{B}}\right), 3.63$ (brs, 1H, 3-H), 3.66 (dd, $J=10.5,6.5 \mathrm{~Hz}, 1 \mathrm{H}$, $\left.4^{\prime}-\mathrm{H}_{\mathrm{A}}\right), 3.73\left(\mathrm{dd}, J=10.5,5.5 \mathrm{~Hz}, 1 \mathrm{H}, 4^{\prime}-\mathrm{H}_{\mathrm{B}}\right), 4.54$ (dd, $\left.J=9.0,7.0 \mathrm{~Hz}, 1 \mathrm{H}, 4-\mathrm{H}\right)$, 4.90 (unter Pyridin-Signal, 1H, 10- $\mathrm{H}_{\mathrm{A}}$ ), 4.96 (brs, 1H, 1- $\left.\mathrm{H}\right), 5.00\left(\mathrm{~m}, 1 \mathrm{H}, 10-\mathrm{H}_{\mathrm{B}}\right.$ ), 5.83 (brs, 1H, 4'-OH), 6.09 (d, J = $3.5 \mathrm{~Hz}, 1 \mathrm{H}, 8-\mathrm{H}), 7.15$ (brd, J = $5.5 \mathrm{~Hz}, 1 \mathrm{H}$, 1-OH), 7.46 (brs, 1H, 3-OH) ppm.

${ }^{13} \mathrm{C}-\mathrm{NMR}\left(150 \mathrm{MHz}, \mathrm{D}_{5}\right.$-Pyridin, 35ㅇ $\left.\mathrm{C}\right): \delta_{\mathrm{C}}=17.19$ (q, C-5'), 20.08 (q, C-11), 27.69 (t, C-2'), 30.41 (t, C-1'), 32.25 (t, C-5), 36.77 (d, C-3’), 64.03 (d, C-3), 64.21 (s, C-2), 66.90 (d, C-4), 67.33 (t, C-4'), 70.52 (d, C-1), 97.53 (d, C-8), 103.05 (s, C-6), 113.56 (t, C-10), 140.17 (s, C-9), 204.18 (s, C-7) ppm.

${ }^{1} \mathrm{H}-\mathrm{NMR}\left(500 \mathrm{MHz}, \mathrm{CD}_{3} \mathrm{OD}\right): \delta_{\mathrm{H}}=0.91\left(\mathrm{~d}, \mathrm{~J}=6.5,3 \mathrm{H}, 5^{\prime}-\mathrm{H}_{3}\right), 1.18\left(\mathrm{~m}, 1 \mathrm{H}, 2^{\prime}-\mathrm{H}_{\mathrm{A}}\right)$, $1.52\left(\mathrm{~m}, 1 \mathrm{H}, 1^{\prime}-\mathrm{H}_{\mathrm{A}}\right), 1.55\left(\mathrm{~m}, 1 \mathrm{H}, 3^{\prime}-\mathrm{H}\right), 1.61\left(\mathrm{~m}, 1 \mathrm{H}, 2^{\prime}-\mathrm{H}_{\mathrm{B}}\right), 1.71\left(\mathrm{~s}, 3 \mathrm{H}, 11-\mathrm{H}_{3}\right)$, 2.06 (ddd, $\left.J=13.5,11.5,4.5 \mathrm{~Hz}, 1 \mathrm{H}, 1^{\prime}-\mathrm{H}_{\mathrm{B}}\right), 2.31\left(\mathrm{~m}, 1 \mathrm{H}, 5-\mathrm{H}_{\mathrm{A}}\right), 2.33(\mathrm{~m}, 1 \mathrm{H}$, 5- $\mathrm{H}_{\mathrm{B}}$ ), 3.02 (brs, 1H, 3-H), 3.34 (dd, $\left.J=10.5,6.5 \mathrm{~Hz}, 1 \mathrm{H}, 4^{\prime}-\mathrm{H}_{\mathrm{A}}\right), 3.42$ (dd, $\left.J=10.5,5.5 \mathrm{~Hz}, 1 \mathrm{H}, 4^{\prime}-\mathrm{H}_{\mathrm{B}}\right), 3.91$ (dd, $\left.J=9.0,7.0 \mathrm{~Hz}, 1 \mathrm{H}, 4-\mathrm{H}\right), 4.34(\mathrm{~s}, 1 \mathrm{H}, 1-\mathrm{H})$, $4.81\left(\mathrm{~m}, 1 \mathrm{H}, 10-\mathrm{H}_{\mathrm{A}}\right), 4.89\left(\mathrm{~m}, 1 \mathrm{H}, 10-\mathrm{H}_{\mathrm{B}}\right), 5.95(\mathrm{~d}, J=3.5 \mathrm{~Hz}, 1 \mathrm{H}, 8-\mathrm{H}) \mathrm{ppm}$. 
${ }^{13}$ C-NMR (125 MHz, CD ${ }_{3} \mathrm{OD}$ ): $\delta_{\mathrm{C}}=17.08$ (q, C-5'), 20.11 (q, C-11), 27.91 (t, C-2'), 30.66 (t, C-1'), 31.82 (t, C-5), 37.03 (d, C-3'), 64.16 (d, C-3), 64.56 (s, C-2), 67.38 (d, C-4), 68.13 (t, C-4'), 70.92 (d, C-1), 98.57 (d, C-8), 102.04 (s, C-6), 114.05 (t, C-10), 140.70 (s, C-9), 205.21 (s, C-7) ppm.

\section{Lupallen C (무)}

$\mathrm{C}_{18} \mathrm{H}_{28} \mathrm{O}_{6}(340.42)$

$\mathbf{R}_{\mathbf{f}}$-Wert: 0.29 (Chloroform/Methanol 9:1)

0.42 (Cyclohexan/Essigester/Methanol 5:10:2)

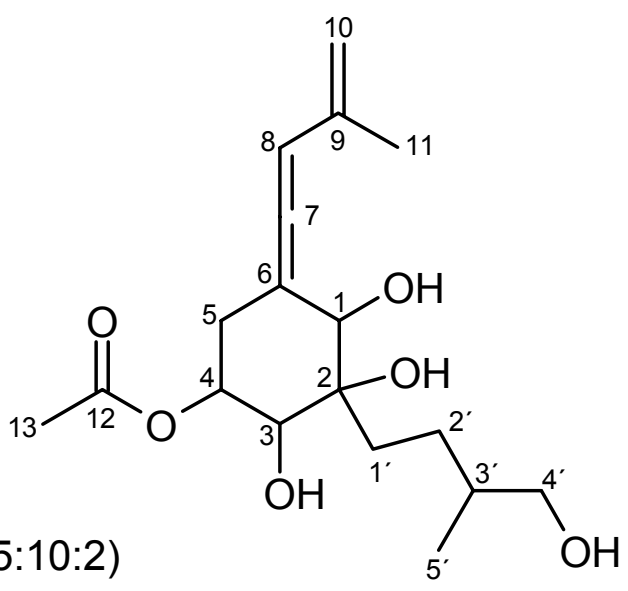

0.39 (Essigester/Methanol 19:1).

Anfärbeverhalten: pink (Anis), blau (Ehrlich), gelb-braun (Orcin).

$[\alpha]_{D}^{20}=+40^{\circ}(\mathrm{c}=1.0 \mathrm{mg} / \mathrm{mL}$ in Methanol).

ESI-MS: $\mathrm{m} / \mathrm{z}=363.6[\mathrm{M}+\mathrm{Na}]^{+}, 703.1[2 \mathrm{M}+\mathrm{Na}]^{+}, 339.6[\mathrm{M}-\mathrm{H}]^{-}$.

HR-ESI-MS: 341.1962 gefunden wie berechnet für $\mathrm{C}_{18} \mathrm{H}_{29} \mathrm{O}_{6}$, 363.1778 gefunden wie berechnet für $\mathrm{C}_{18} \mathrm{H}_{28} \mathrm{O}_{6} \mathrm{Na}$.

IR (KBr): $\tilde{v}=3409,2958,2925,2824,1956,1718,1628,1453,1433,1378,1259$, $1119,1033,982,886 \mathrm{~cm}^{-1}$.

UV $(\mathrm{MeOH}) \quad: \lambda_{\max }(\varepsilon)=220$ (12524), 233 sh (8090);

$(\mathrm{MeOH} / \mathrm{HCl}) \quad: \lambda_{\max }(\varepsilon)=220$ (12504), $231 \mathrm{sh}(8563) ;$

$(\mathrm{MeOH} / \mathrm{NaOH}): \lambda_{\max }(\varepsilon)=221$ (11906), $291(391) \mathrm{nm}$.

CD $(\mathrm{MeOH}): \lambda_{\max }[\Theta]=213$ (18810), 236 (602), 253 (3065) nm.

${ }^{1} \mathrm{H}-\mathrm{NMR}\left(600 \mathrm{MHz}, \mathrm{D}_{5}\right.$-Pyridin, $\left.35^{\circ} \mathrm{C}\right): \delta_{\mathrm{C}}=1.10\left(\mathrm{~d}, J=7.0 \mathrm{~Hz}, 3 \mathrm{H}, 5^{\prime}-\mathrm{H}_{3}\right), 1.79(\mathrm{~m}$, $\left.1 \mathrm{H}, 2^{\prime}-\mathrm{H}_{\mathrm{A}}\right), 1.85\left(\mathrm{~s}, 3 \mathrm{H}, 11-\mathrm{H}_{3}\right), 1.89\left(\mathrm{~m}, 1 \mathrm{H}, 3^{\prime}-\mathrm{H}\right), 1.97\left(\mathrm{~s}, 3 \mathrm{H}, 13-\mathrm{H}_{3}\right), 2.25$ (tt, 
$\left.J=12.5,4.5 \mathrm{~Hz}, 1 \mathrm{H}, 2^{\prime}-\mathrm{H}_{\mathrm{B}}\right), 2.44\left(\mathrm{ddd}, J=13.0,12.5,4.5 \mathrm{~Hz}, 1 \mathrm{H}, 1^{\prime}-\mathrm{H}_{\mathrm{A}}\right), 2.52$ (td, $\left.J=12.5,4.5 \mathrm{~Hz}, 1 \mathrm{H}, 1^{\prime}-\mathrm{H}_{\mathrm{B}}\right), 2.75\left(\mathrm{dd}, J=12.5,5.0 \mathrm{~Hz}, 1 \mathrm{H}, 5-\mathrm{H}_{\mathrm{A}}\right), 3.40$ (td, $\left.J=12.5,4.0 \mathrm{~Hz}, 1 \mathrm{H}, 5-\mathrm{H}_{\mathrm{B}}\right), 3.70$ (dd, $\left.J=10.0,6.5 \mathrm{~Hz}, 1 \mathrm{H}, 4^{\prime}-\mathrm{H}_{\mathrm{A}}\right), 3.78$ (dd, $J=10.5,6.0 \mathrm{~Hz}, 1 \mathrm{H}, 4^{\prime}-\mathrm{H}_{\mathrm{B}}$ ), 4.48 (brs, 1H, 3-H), 4.57 (brs, 1H, 1-H), 4.85 (quin, $\left.J=0.5 \mathrm{~Hz}, 1 \mathrm{H}, 10-\mathrm{H}_{\mathrm{A}}\right), 4.96\left(\mathrm{~m}, 1 \mathrm{H}, 10-\mathrm{H}_{\mathrm{B}}\right), 6.00(\mathrm{ddd}, J=12.0,5.0,2.5 \mathrm{~Hz}, 1 \mathrm{H}$, 4-H), 6.05 (d, J = 4.0 Hz, 1H, 8-H), 6.39 (brs, 1H, 2-OH), 6.47 (brd, J = 5.5 Hz, 1H, 1-OH), 7.00 (brs, 1H, 3-OH) ppm.

${ }^{13}$ C-NMR (150 MHz, D - Pyridin, 35 C): $\delta_{\mathrm{C}}=17.32$ (q, C-5'), 19.72 (q, C-11), 20.99 (q, C-13), 26.34 (t, C-2'), 26.34 (t, C-5), 34.00 (t, C-1'), 37.18 (d, C-3'), 67.68 (t, C-4'), 72.68 (d, C-4), 74.22 (d, C-3), 74.73 (s, C-2), 76.94 (d, C-1), 97.30 (d, C-8), 103.95 (s, C-6), 113.67 (t, C-10), 139.95 (s, C-9), 170.30 (s, C-12), 204.38 (s, C-7) ppm.

\section{Lupallen D ( $\underline{(90 \mathrm{a} / \mathrm{b}})$}

$\mathrm{C}_{18} \mathrm{H}_{26} \mathrm{O}_{5}(322.40)$

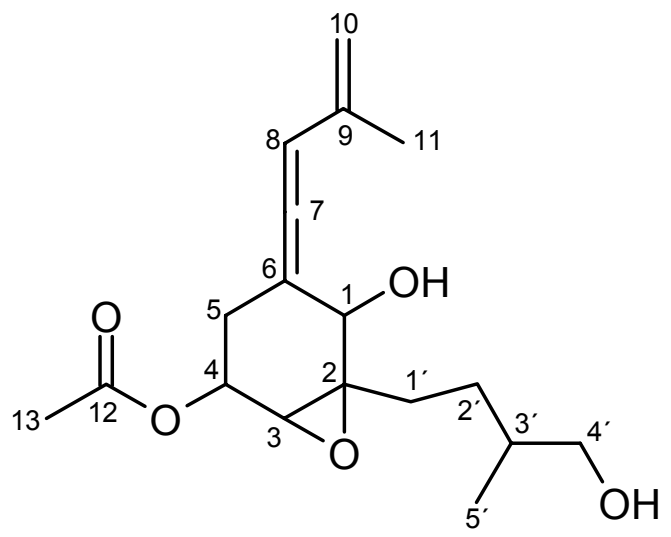

$\mathbf{R}_{\mathbf{f}}$-Wert: 0.49 (Chloroform/Methanol 9:1)

0.53 (Cyclohexan/Essigester/Methanol 5:10:2)

0.51 (Essigester/Methanol 19:1).

Anfärbeverhalten: pink (Anis), blau (Ehrlich), gelb-braun (Orcin).

$[\alpha]_{D}^{20}=+62^{\circ}(\mathrm{c}=1.0 \mathrm{mg} / \mathrm{mL}$ in Methanol $)$.

ESI-MS: $\mathrm{m} / \mathrm{z}=354.2[\mathrm{M}+\mathrm{Na}]^{+}$.

IR $(\mathrm{KBr}): \widetilde{v}=3427,2928,2873,1957,1730,1644,1628,1455,1431,1375,1242$, $1033,984,893 \mathrm{~cm}^{-1}$.

UV $(\mathrm{MeOH}) \quad: \lambda_{\max }(\varepsilon)=200$ (8923), 219 (17378); 
$(\mathrm{MeOH} / \mathrm{HCl}) \quad: \lambda_{\max }(\varepsilon)=200$ (9034); 218 (16915);

$(\mathrm{MeOH} / \mathrm{NaOH}): \lambda_{\max }(\varepsilon)=204(24708), 212(19201) \mathrm{nm}$.

CD $(\mathrm{MeOH}): \lambda_{\max }[\Theta]=211$ (6038), 213 (5557), 216 (6513), 228 (-1384), 249 (5905) $\mathrm{nm}$.

${ }^{1} \mathrm{H}$-NMR $\left(600 \mathrm{MHz}, \mathrm{D}_{5}\right.$-Pyridin, $\left.35^{\circ} \mathrm{C}\right): \delta_{\mathrm{H}}=1.03 / 1.04\left(\mathrm{~d}, J=6.5 \mathrm{~Hz}, 3 \mathrm{H}, 5^{\prime}-\mathrm{H}_{3}\right)$, $1.52 / 1.50$ (dddd, $J=12.5,12.0,7.5,4.5 \mathrm{~Hz}, 1 \mathrm{H}, 2^{\prime}-\mathrm{H}_{\mathrm{A}}$ ), $1.78 / 1.89$ (q, $J=0.5 \mathrm{~Hz}$, $\left.3 \mathrm{H}, 11-\mathrm{H}_{3}\right), 1.81\left(\mathrm{~m}, 1 \mathrm{H}, 3^{\prime}-\mathrm{H}\right), 1.90\left(\mathrm{ddd}, J=14.0,12.5,4.5 \mathrm{~Hz}, 1 \mathrm{H}, 1^{\prime}-\mathrm{H}_{\mathrm{A}}\right)$, 2.04/2.05 (s, 3H, 13- $\left.\mathrm{H}_{3}\right), 2.07\left(\mathrm{~m}, 1 \mathrm{H}, 2{ }^{\prime}-\mathrm{H}_{\mathrm{B}}\right), 2.49 / 2.48$ (ddd, $J=14.0,12.0$, $5.0 \mathrm{~Hz}, 1 \mathrm{H}, 1^{\prime}-\mathrm{H}_{\mathrm{B}}$ ), 2.74/2.71 (dd, $J=12.5,7.0 \mathrm{~Hz}, 1 \mathrm{H}, 5-\mathrm{H}_{\mathrm{A}}$ ), $2.87 / 2.90$ (ddd, $\left.J=12.5,9.0,3.0 \mathrm{~Hz}, 1 \mathrm{H}, 5-\mathrm{H}_{\mathrm{B}}\right), 3.39 / 3.38(\mathrm{~d} / \mathrm{brs}, J=1.0 \mathrm{~Hz}, 1 \mathrm{H}, 3-\mathrm{H}), 3.65 / 3.66$ (dd, $J=10.0,6.0 \mathrm{~Hz}, 1 \mathrm{H}, 4^{\prime}-\mathrm{H}_{\mathrm{A}}$ ), $3.72\left(\mathrm{dd}, J=10.0,6.0 \mathrm{~Hz}, 1 \mathrm{H}, 4^{\prime}-\mathrm{H}_{\mathrm{B}}\right), 4.86 / 4.88$ (quin, $J=1.0 \mathrm{~Hz}, 1 \mathrm{H}, 10-\mathrm{H}_{\mathrm{A}}$ ), 4.95/4.91 (brs, $\left.1 \mathrm{H}, 1-\mathrm{H}\right), 5.00 / 4.99\left(\mathrm{~m}, 1 \mathrm{H}, 10-\mathrm{H}_{\mathrm{B}}\right.$ ), $5.43 / 5.36$ (ddd, $J=8.5,7.0,0.5 \mathrm{~Hz} / \mathrm{dd} J=10.0,7.0 \mathrm{~Hz}, 1 \mathrm{H}, 4-\mathrm{H}$ ), 5.83 (brs, $1 \mathrm{H}$, 4'-OH), 6.13/6.08 (d, $J=3.5 / 3.0 \mathrm{~Hz}, 1 \mathrm{H}, 8-\mathrm{H}), 7.45 / 7.61$ (brs, $1 \mathrm{H}, 1-\mathrm{OH}$ ) ppm.

${ }^{13}$ C-NMR (150 MHz, D 5 -Pyridin, $35^{\circ} \mathrm{C}$ ): $\delta_{\mathrm{C}}=17.23 / 17.20$ (q, C-5'), 19.70/20.06 (q, C-11), 20.87 (q, C-13), 27.74/27.58 (t, C-2'), 28.18/28.08 (t, C-5), 30.19/30.08 (t, $\left.\mathrm{C}^{\prime} \mathbf{1}^{\prime}\right), \quad 36.75 / 36.80$ (d, C-3'), 60.56/60.28 (d, C-3), 64.30/64.17 (s, C-2), 67.32/67.36 (t, C-4'), 68.62/68.99 (d, C-4), 69.33/69.95 (d, C-1), 98.65/98.08 (d, C-8), 101.90/101.49 (s, C-6), 114.20 (t, C-10), 139.72/139.82 (s, C-9), 170.09 (s, C-12), 204.10/204.42 (s, C-7) ppm.

\section{Lupallen E (91)}

$\mathrm{C}_{16} \mathrm{H}_{26} \mathrm{O}_{5}(298.38)$

$\mathbf{R}_{\mathbf{f}}$-Wert: 0.09 (Chloroform/Methanol 9:1)

0.29 (Cyclohexan/Essigester/Methanol 5:10:2)

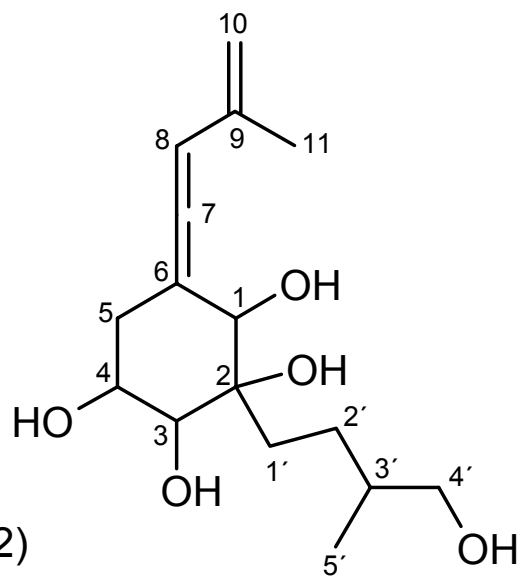

0.24 (Essigester/Methanol 19:1). 
Anfärbeverhalten: pink (Anis), blau (Ehrlich), gelb-braun (Orcin).

$[\alpha]_{D}^{20}=+30^{\circ}(\mathrm{c}=1.0 \mathrm{mg} / \mathrm{mL}$ in Methanol $)$.

ESI-MS: $\mathrm{m} / \mathrm{z}=321.3[\mathrm{M}+\mathrm{Na}]^{+}, 619.0[2 \mathrm{M}+\mathrm{Na}]^{+}, 297.2[\mathrm{M}-\mathrm{H}]^{-}, 595.1[2 \mathrm{M}-\mathrm{H}]^{-}$.

IR $(\mathrm{KBr}): \widetilde{v}=3405,2954,2926,2868,1954,1629,1454,1430,1384,1240,1113$, $1033,955,882 \mathrm{~cm}^{-1}$.

UV $\left(\mathrm{MeOH} \quad: \lambda_{\max }(\varepsilon)=220\right.$ (9917), 232 sh (7199), 277 (245);

$(\mathrm{MeOH} / \mathrm{HCl}) \quad: \lambda_{\max }(\varepsilon)=220$ (9761), 233 sh (6618);

$(\mathrm{MeOH} / \mathrm{NaOH}): \lambda_{\max }(\varepsilon)=221$ (8946), 233 (6297) nm.

CD $(\mathrm{MeOH}): \lambda_{\max }[\Theta]=201$ (4819), 203 (-136), 215 (6410), 234 (-2732), 253 (2863) $\mathrm{nm}$.

${ }^{1} \mathrm{H}-\mathrm{NMR}\left(600 \mathrm{MHz}, \mathrm{D}_{5}-\mathrm{Py}\right.$ ridin, $\left.35^{\circ} \mathrm{C}\right): \delta_{\mathrm{H}}=1.13\left(\mathrm{~d}, \mathrm{~J}=6.5 \mathrm{~Hz}, 3 \mathrm{H}, 5^{\prime}-\mathrm{H}_{3}\right), 1.84(\mathrm{~m}$, $\left.1 \mathrm{H}, 2^{\prime}-\mathrm{H}_{\mathrm{A}}\right), 1.88\left(\mathrm{~s}, 3 \mathrm{H}, 11-\mathrm{H}_{3}\right), 1.93\left(\mathrm{~m}, 1 \mathrm{H}, 3^{\prime}-\mathrm{H}\right), 2.28(\mathrm{tt}, J=12.5,5.0 \mathrm{~Hz}, 1 \mathrm{H}$, $\left.1^{\prime}-\mathrm{H}_{\mathrm{A}}\right), 2.54\left(\mathrm{~m}, 2 \mathrm{H}, 1^{\prime}-\mathrm{H}_{\mathrm{B}}, 2^{\prime}-\mathrm{H}_{\mathrm{B}}\right), 2.88\left(\mathrm{dd}, J=13.0,5.0 \mathrm{~Hz}, 1 \mathrm{H}, 5-\mathrm{H}_{\mathrm{A}}\right), 3.42(\mathrm{td}$, $\left.J=12.5,4.0 \mathrm{~Hz}, 1 \mathrm{H}, 5-\mathrm{H}_{\mathrm{B}}\right), 3.72\left(\mathrm{dd}, J=10.0,5.0 \mathrm{~Hz}, 1 \mathrm{H}, 4^{\prime}-\mathrm{H}_{\mathrm{A}}\right), 3.82$ (dd, $\left.J=10.0,5.0 \mathrm{~Hz}, 1 \mathrm{H}, 4^{\prime}-\mathrm{H}_{\mathrm{B}}\right), 4.47$ (brs, 1H, 3-H), 4.60 (d, J = 8.0 Hz, 1H, 1-H), 4.85 (quin, $J=0.5 \mathrm{~Hz}, 1 \mathrm{H}, 10-\mathrm{H}_{\mathrm{A}}$ ), $4.97\left(\mathrm{~m}, 2 \mathrm{H}, 4-\mathrm{H}, 10-\mathrm{H}_{\mathrm{B}}\right.$ ), 5.83 (brt, 1H, 4'-OH), 6.04 (s, 1H, 2-OH), 6.09 (d, J = 4.0 Hz, 1H, 8-H), 6.13 (brd, J = $7.5 \mathrm{~Hz}, 1 \mathrm{H}, 4-\mathrm{OH}), 6.36$ $(\mathrm{d}, J=8.0 \mathrm{~Hz}, 1 \mathrm{H}, 1-\mathrm{OH}), 6.75(\mathrm{~d}, J=5.5 \mathrm{~Hz}, 1 \mathrm{H}, 3-\mathrm{OH}) \mathrm{ppm}$.

${ }^{13} \mathrm{C}-\mathrm{NMR}\left(150 \mathrm{MHz}, \mathrm{D}_{5}\right.$-Pyridin, $\left.35^{\circ} \mathrm{C}\right): \delta_{\mathrm{C}}=17.52$ (q, C-5'), 19.92 (q, C-11), 26.54 (t, C-2'), 30.48 (t, C-5), 34.55 (t, C-1'), 37.42 (d, C-3'), 67.89 (t, C-4'), 68.6 (d, C-4), 74.76 (s, C-2), 77.20 (d, C-3), 77.26 (d, C-1), 96.97 (d, C-8), 105.26 (s, C-6), 113.34 (t, C-10), 140.46 (s, C-9), 204.21 (s, C-7) ppm.

${ }^{1} \mathrm{H}-\mathrm{NMR}\left(600 \mathrm{MHz}, \mathrm{CD}_{3} \mathrm{OD}\right): \delta_{\mathrm{H}}=0.95\left(\mathrm{~d}, J=6.5 \mathrm{~Hz}, 3 \mathrm{H}, 5^{\prime}-\mathrm{H}_{3}\right), 1.28(\mathrm{~m}, 1 \mathrm{H}$, $\left.2^{\prime}-\mathrm{H}_{\mathrm{A}}\right), 1.58\left(\mathrm{~m}, 2 \mathrm{H}, 2^{\prime}-\mathrm{H}_{\mathrm{B}}, 3^{\prime}-\mathrm{H}\right), 1.78\left(\mathrm{~s}, 3 \mathrm{H}, 11-\mathrm{H}_{3}\right), 1.82$ (ddd, $J=14.0,12.0$, $\left.4.5 \mathrm{~Hz}, 1 \mathrm{H}, 1^{\prime}-\mathrm{H}_{\mathrm{A}}\right), 1.92$ (ddd, $\left.J=14.0,12.5,4.5 \mathrm{~Hz}, 1 \mathrm{H}, 1^{\prime}-\mathrm{H}_{\mathrm{B}}\right), 2.24$ (dd, $\left.J=13.0,5.0 \mathrm{~Hz}, 1 \mathrm{H}, 5-\mathrm{H}_{\mathrm{A}}\right), 2.66\left(\mathrm{td}, J=12.5,4.0 \mathrm{~Hz}, 1 \mathrm{H}, 5-\mathrm{H}_{\mathrm{B}}\right), 3.36(\mathrm{dd}$, $\left.J=10.5,6.5 \mathrm{~Hz}, 1 \mathrm{H}, 4^{\prime}-\mathrm{H}_{\mathrm{A}}\right), 3.47$ (dd, $\left.J=10.5,5.5 \mathrm{~Hz}, 1 \mathrm{H}, 4^{\prime}-\mathrm{H}_{\mathrm{B}}\right), 3.62$ (brs, $1 \mathrm{H}$, 3-H), 3.86 (d, J=1.0 Hz, 1H, 1-H), 4.03 (ddd, $J=12.0,5.5,3.0 \mathrm{~Hz}, 1 \mathrm{H}, 4-\mathrm{H}$ ), 4.81 
(quin, $\left.J=1.0 \mathrm{~Hz}, 1 \mathrm{H}, 10-\mathrm{H}_{\mathrm{A}}\right), 4.90\left(\mathrm{~m}, 1 \mathrm{H}, 10-\mathrm{H}_{\mathrm{B}}\right), 5.94(\mathrm{~d}, J=4.0 \mathrm{~Hz}, 1 \mathrm{H}, 8-\mathrm{H})$ ppm.

${ }^{13}$ C-NMR (150 MHz, CD ${ }_{3} \mathrm{OD}$ ): $\delta_{\mathrm{C}}=17.21$ (q, C-5'), 19.94 (q, C-11), 26.24 (t, C-2'), 29.86 (t, C-5), 34.01 (t, C-1'), 37.47 (d, C-3'), 68.45 (t, C-4'), 68.96 (d, C-4), 75.07 (s, C-2), 76.66 (d, C-3), 76.98 (d, C-1), 97.90 (d, C-8), 103.76 (s, C-6), 113.85 (t, C-10), 140.95 (s, C-9), 204.97 (s, C-7) ppm.

\section{Lupallen $F_{1}(\underline{92})$}

$\mathrm{C}_{16} \mathrm{H}_{24} \mathrm{O}_{4}(280.36)$

$\mathbf{R}_{\mathbf{f}}$-Wert: 0.22 (Chloroform/Methanol 9:1)

0.34 (Cyclohexan/Essigester/Methanol 5:10:2)

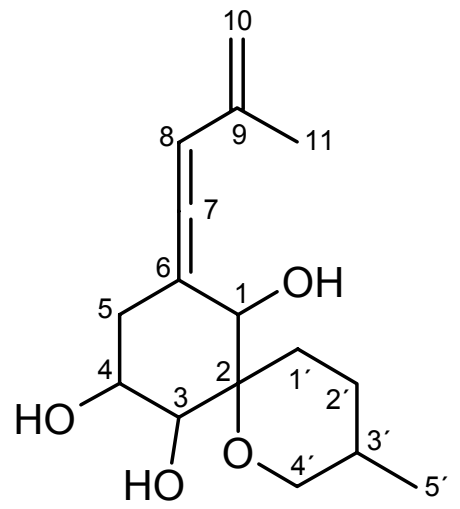

0.27 (Essigester/Methanol 19:1).

Anfärbeverhalten: pink (Anis), blau (Ehrlich), braun (Orcin).

$[\alpha]_{D}^{20}=+45^{\circ}(\mathrm{c}=1.0 \mathrm{mg} / \mathrm{mL}$ in Methanol $)$.

ESI-MS: $\mathrm{m} / \mathrm{z}=303.2[\mathrm{M}+\mathrm{Na}]^{+}, 583.0[2 \mathrm{M}+\mathrm{Na}]^{+}$.

IR $(\mathrm{KBr}): \tilde{v}=3422,2953,2929,2865,1954,1629,1457,1384,1286,1101,1055$, $1030,780 \mathrm{~cm}^{-1}$.

UV $(\mathrm{MeOH}) \quad: \lambda_{\max }(\varepsilon)=220$ (17620), $283(2002)$

$(\mathrm{MeOH} / \mathrm{HCl}) \quad: \lambda_{\max }(\varepsilon)=221$ (16182), 284 (1589);

$(\mathrm{MeOH} / \mathrm{NaOH}): \lambda_{\max }(\varepsilon)=217(18248), 284(1905) \mathrm{nm}$.

CD $(\mathrm{MeOH}): \lambda_{\max }[\Theta]=201$ (9079), 217 (19953), $247(-10981) \mathrm{nm}$.

${ }^{1} \mathrm{H}-\mathrm{NMR}\left(600 \mathrm{MHz}, \mathrm{D}_{5}\right.$-Pyridin, $\left.35^{\circ} \mathrm{C}\right): \delta_{\mathrm{H}}=0.67\left(\mathrm{~d}, J=6.5 \mathrm{~Hz}, 3 \mathrm{H}, 5^{\prime}-\mathrm{H}_{3}\right), 1.31$ (qd, $\left.J=12.5,4.0 \mathrm{~Hz}, 1 \mathrm{H}, 2^{\prime}-\mathrm{H}_{\mathrm{A}}\right), 1.63\left(\mathrm{~m}, 1 \mathrm{H}, 2^{\prime} \mathrm{H}_{\mathrm{B}}\right), 1.69\left(\mathrm{~m}, 1 \mathrm{H}, 3^{\prime}-\mathrm{H}\right), 1.75$ (dt, $\left.J=14.0,3.5 \mathrm{~Hz}, 1 \mathrm{H}, 1^{\prime}-\mathrm{H}_{\mathrm{A}}\right), 1.89\left(\mathrm{~s}, 3 \mathrm{H}, 11-\mathrm{H}_{3}\right), 2.46(\mathrm{td}, J=13.5,5.0 \mathrm{~Hz}, 1 \mathrm{H}$, 
$1^{\prime}-\mathrm{H}_{\mathrm{B}}$ ), 2.90 (dd, $J=13.0,5.5 \mathrm{~Hz}, 1 \mathrm{H}, 5-\mathrm{H}_{\mathrm{A}}$ ), 3.23 (ddd, $J=13.0,11.5,4.0 \mathrm{~Hz}, 1 \mathrm{H}$, $\left.5-\mathrm{H}_{\mathrm{B}}\right), 3.50\left(\mathrm{t}, J=11.5 \mathrm{~Hz}, 1 \mathrm{H}, 4^{\prime}-\mathrm{H}_{\mathrm{A}}\right), 3.70$ (ddd, $J=11.5,4.5,2.0 \mathrm{~Hz}, 1 \mathrm{H}, 4^{\prime}-\mathrm{H}_{\mathrm{B}}$ ), 4.13 (ddd, $J=11.0,9.0,5.5 \mathrm{~Hz}, 1 \mathrm{H}, 4-\mathrm{H}$ ), 4.56 (d, $J=9.0 \mathrm{~Hz}, 1 \mathrm{H}, 3-\mathrm{H}$ ), 4.91 (quin, $\left.J=1.0 \mathrm{~Hz}, 1 \mathrm{H}, 10-\mathrm{H}_{\mathrm{A}}\right), 5.07\left(\mathrm{~m}, 1 \mathrm{H}, 10-\mathrm{H}_{\mathrm{B}}\right), 5.15(\mathrm{~s}, 1 \mathrm{H}, 1-\mathrm{H}), 5.95$ (brs, $1 \mathrm{H}$, $1-\mathrm{OH}), 6.17$ (d, J = 4.0 Hz, 1H, 8-H), 6.20 (brs, 1H, 4-OH), 6.34 (brs, 1H, 4'-OH) ppm.

${ }^{13}$ C-NMR (150 MHz, D - Pyridin, 35ㅇ $): \delta_{C}=17.36$ (q, C-5'), 19.87 (q, C-11), 24.15 (t, C-1'), 27.99 (t, C-2'), 30.81 (d, C-3'), 33.76 (t, C-5), 68.16 (t, C-4'), 69.60 (d, C-1), 70.73 (d, C-4), 76.42 (d, C-3), 78.60 (s, C-2), 97.08 (d, C-8), 103.80 (s, C-6), 114.04 (t, C-10), 140.05 (s, C-9), 203.84 (s, C-7) ppm.

\section{Lupallen $F_{2}(\underline{93})$}

$\mathrm{C}_{16} \mathrm{H}_{24} \mathrm{O}_{4}(280.36)$

$\mathbf{R}_{\mathbf{f}}$-Wert: 0.22 (Chloroform/Methanol 9:1)

0.32 (Cyclohexan/Essigester/Methanol 5:10:2)

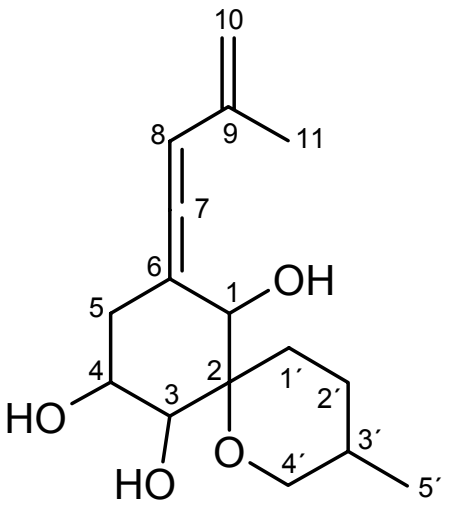
0.24 (Essigester/Methanol 19:1).

Anfärbeverhalten: pink (Anis), blau (Ehrlich), gelb-braun (Orcin).

$[\alpha]_{D}^{20}=+82^{\circ}(\mathrm{c}=1.0 \mathrm{mg} / \mathrm{mL}$ in Methanol $)$.

ESI-MS: $\mathrm{m} / \mathrm{z}=303.2[\mathrm{M}+\mathrm{Na}]^{+}, 583.0[2 \mathrm{M}+\mathrm{Na}]^{+}$.

IR $(\mathrm{KBr}): \tilde{v}=3416,2927,2867,1956,1624,1454,1383,1286,1213,1155,1101$, $1054,880,859 \mathrm{~cm}^{-1}$.

UV $(\mathrm{MeOH}) \quad: \lambda_{\max }(\varepsilon)=220$ (8955), 284 (1112);

$(\mathrm{MeOH} / \mathrm{HCl}) \quad: \lambda_{\max }(\varepsilon)=222(7581), 286(683)$;

$(\mathrm{MeOH} / \mathrm{NaOH}): \lambda_{\max }(\varepsilon)=222(7661), 286(652) \mathrm{nm}$. 
CD $(\mathrm{MeOH}): \lambda_{\max }[\Theta]=205$ (2013), 218 (6940), $244(-4442), 262(-1424), 283$ $(-1892) \mathrm{nm}$.

${ }^{1} \mathrm{H}-\mathrm{NMR}\left(600 \mathrm{MHz}, \mathrm{D}_{5}\right.$-Pyridin, $\left.35^{\circ} \mathrm{C}\right): \delta_{\mathrm{H}}=0.67\left(\mathrm{~d}, J=6.5 \mathrm{~Hz}, 3 \mathrm{H}, 5^{\prime}-\mathrm{H}_{3}\right), 1.29$ (qd, $\left.J=12.5,4.0 \mathrm{~Hz}, 1 \mathrm{H}, 2^{\prime}-\mathrm{H}_{\mathrm{A}}\right), 1.62\left(\mathrm{~m}, 1 \mathrm{H}, 2^{\prime} \mathrm{H}_{\mathrm{B}}\right), 1.72\left(\mathrm{~m}, 1 \mathrm{H}, 3^{\prime}-\mathrm{H}\right), 1.85(\mathrm{~s}$, $\left.3 \mathrm{H}, 11-\mathrm{H}_{3}\right), 1.88\left(\mathrm{~m}, 1 \mathrm{H}, 1^{\prime}-\mathrm{H}_{\mathrm{A}}\right), 2.53\left(\mathrm{td}, J=14.0,4.5 \mathrm{~Hz}, 1 \mathrm{H}, 1^{\prime}-\mathrm{H}_{\mathrm{B}}\right), 2.87$ (dd, $J=13.0,5.5 \mathrm{~Hz}, 1 \mathrm{H}, 5-\mathrm{H}_{\mathrm{A}}$ ), 3.21 (ddd, $\left.J=12.5,12.0,4.5 \mathrm{~Hz}, 1 \mathrm{H}, 5-\mathrm{H}_{\mathrm{B}}\right), 3.51$ (t, $J=11.5 \mathrm{~Hz}, 1 \mathrm{H}, 4^{\prime}-\mathrm{H}_{\mathrm{A}}$ ), 3.73 (ddd, $J=11.5,4.5,2.0 \mathrm{~Hz}, 1 \mathrm{H}, 4^{\prime}-\mathrm{H}_{\mathrm{B}}$ ), 4.19 (ddd, $J=11.5,9.5,5.5 \mathrm{~Hz}, 1 \mathrm{H}, 4-\mathrm{H}), 4.60(\mathrm{~d}, J=9.5 \mathrm{~Hz}, 1 \mathrm{H}, 3-\mathrm{H}$ ), 4.93 (quin, $J=1.0 \mathrm{~Hz}, 1 \mathrm{H}, 10-\mathrm{H}_{\mathrm{A}}$ ), 5.07 (brs, $1 \mathrm{H}, 10-\mathrm{H}_{\mathrm{B}}$ ), 5.17 (s, 1H, 1-H), 6.12 (brs, $1 \mathrm{H}$, 1-OH), 6.16 (d, $J=4.5 \mathrm{~Hz}, 1 \mathrm{H}, 8-\mathrm{H}$ ), 6.24 (brs, 1H, 4-OH), 6.37 (brs, $1 \mathrm{H}, 4^{\prime}-\mathrm{OH}$ ) ppm.

${ }^{13} \mathrm{C}$-NMR (150 MHz, D 5 -Pyridin, 35ㅇ): $\delta_{\mathrm{C}}=17.36$ (q, C-5'), 20.15 (q, C-11), 24.06 (t, C-1'), 27.87 (t, C-2'), 30.95 (d, C-3'), 33.78 (t, C-5), 68.09 (t, C-4'), 69.04 (d, C1), 70.74 (d, C-4), 76.37 (d, C-3), 78.42 (s, C-2), 97.26 (d, C-8), 103.63 (s, C-6), 114.37 (t, C-10), 140.02 (s, C-9), 203.83 (s, C-7) ppm.

Lupaketon A $(\underline{94})$

$\mathrm{C}_{16} \mathrm{H}_{26} \mathrm{O}_{5}(298.38)$

$\mathbf{R}_{\mathbf{f}}$-Wert: 0.20 (Chloroform/Methanol 9:1)

0.35 (Cyclohexan/Essigester/Methanol 5:10:2)

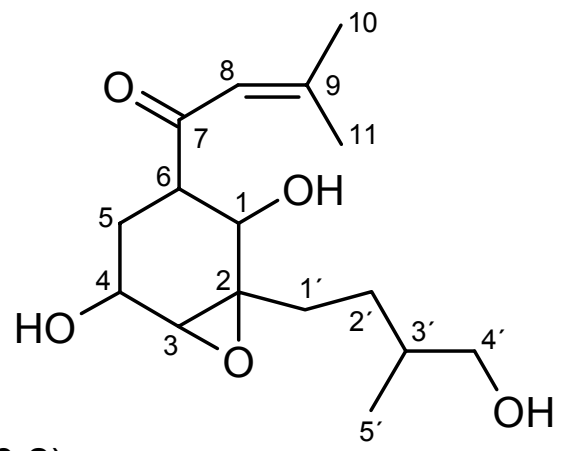

0.31 (Essigester/Methanol 19:1).

Anfärbeverhalten: pink (Anis), blau (Ehrlich), braun (Orcin).

$[\alpha]_{D}^{20}=+13^{\circ}(\mathrm{c}=1.0 \mathrm{mg} / \mathrm{mL}$ in Methanol).

ESI-MS: $\mathrm{m} / \mathrm{z}=321.3[\mathrm{M}+\mathrm{Na}]^{+}, 619.1[2 \mathrm{M}+\mathrm{Na}]^{+}$. 
HR-ESI-MS: 321.16741 gefunden wie berechnet für $\mathrm{C}_{16} \mathrm{H}_{25} \mathrm{O}_{4}$, 303.1566 gefunden wie berechnet für $\mathrm{C}_{16} \mathrm{H}_{26} \mathrm{O}_{5} \mathrm{Na}$.

IR (KBr): $\widetilde{v}=3400,2955,2929,2877,1679,1615,1441,1382,1263,1123,1042$, $841 \mathrm{~cm}^{-1}$.

UV $(\mathrm{MeOH}) \quad \lambda_{\max }(\varepsilon)=238(9440) ;$

$(\mathrm{MeOH} / \mathrm{HCl}) \quad: \lambda_{\max }(\varepsilon)=238(10133)$;

$(\mathrm{MeOH} / \mathrm{NaOH}): \lambda_{\max }(\varepsilon)=237(10137) \mathrm{nm}$.

CD $(\mathrm{MeOH}): \lambda_{\max }[\Theta]=208$ (5335), 236 (-2648), 277 (147), $326(-1629) \mathrm{nm}$.

${ }^{1} \mathrm{H}-\mathrm{NMR}\left(600 \mathrm{MHz}, \mathrm{D}_{5}\right.$-Pyridin, $\left.35^{\circ} \mathrm{C}\right): \delta_{\mathrm{H}}=1.03\left(\mathrm{~d}, \mathrm{~J}=6.5 \mathrm{~Hz}, 3 \mathrm{H}, 5^{\prime}-\mathrm{H}_{3}\right), 1.64$ (brs, 3H, 11- $\left.\mathrm{H}_{3}\right), 1.76\left(\mathrm{~m}, 1 \mathrm{H}, 2^{\prime}-\mathrm{H}_{\mathrm{A}}\right), 1.82\left(\mathrm{~m}, 1 \mathrm{H}, 3^{\prime}-\mathrm{H}\right), 1.96$ (ddd, J = 13.5, 12.0, $\left.5.0 \mathrm{~Hz}, 1 \mathrm{H}, 1^{\prime}-\mathrm{H}_{\mathrm{A}}\right), 2.04\left(\mathrm{~m}, 2 \mathrm{H}, 5-\mathrm{H}_{2}\right), 2.11\left(\mathrm{~m}, 1 \mathrm{H}, 2^{\prime}-\mathrm{H}_{\mathrm{B}}\right), 2.14(\mathrm{~d}, J=1.0 \mathrm{~Hz}, 3 \mathrm{H}$, 10- $\mathrm{H}_{3}$ ), 2.39 (ddd, $J=13.5,12.0,4.5 \mathrm{~Hz}, 1 \mathrm{H}, 1^{\prime}-\mathrm{H}_{\mathrm{B}}$ ), 3.50 (brs, 1H, 3-H), 3.52 (ddd, $J=11.0,9.0,4.5 \mathrm{~Hz}, 1 \mathrm{H}, 6-\mathrm{H}$ ), 3.63 (dd, $J=10.5,6.5 \mathrm{~Hz}, 1 \mathrm{H}, 4^{\prime}-\mathrm{H}_{\mathrm{A}}$ ), 3.72 (dd, $\left.J=10.5,5.5 \mathrm{~Hz}, 1 \mathrm{H}, 4^{\prime}-\mathrm{H}_{\mathrm{B}}\right), 4.67$ (q, $\left.J=2.5 \mathrm{~Hz}, 1 \mathrm{H}, 4-\mathrm{H}\right), 4.90$ (d, J = 9.0 Hz, $1 \mathrm{H}, 1-\mathrm{H}), 6.31(\mathrm{~m}, J=1.0 \mathrm{~Hz}, 1 \mathrm{H}, 8-\mathrm{H}) \mathrm{ppm}$.

${ }^{13}$ C-NMR (150 MHz, D - Pyridin, 35ㅇ $\mathrm{C}$ ): $\delta_{\mathrm{C}}=17.35$ (q, C-5'), 20.66 (q, C-10), 27.21 (q, C-11), 28.68 (t, C-2'), 29.86 (t, C-5), 31.21 (t, C-1'), 36.89 (d, C-3’), 50.92 (d, C-6), 62.29 (d, C-3), 64.52 (s, C-2), 64.76 (d, C-4), 67.39 (t, C-4'), 67.99 (d, C-1), 124.63 (d, C-8), 154.83 (s, C-9), 202.65 (s, C-7) ppm.

Lupaketon B $(\underline{95})$

$\mathrm{C}_{16} \mathrm{H}_{24} \mathrm{O}_{5}(296.36)$

$\mathbf{R}_{\mathbf{f}}$-Wert: 0.22 (Chloroform/Methanol 9:1)

0.33 (Cyclohexan/Essigester/Methanol 5:10:2)

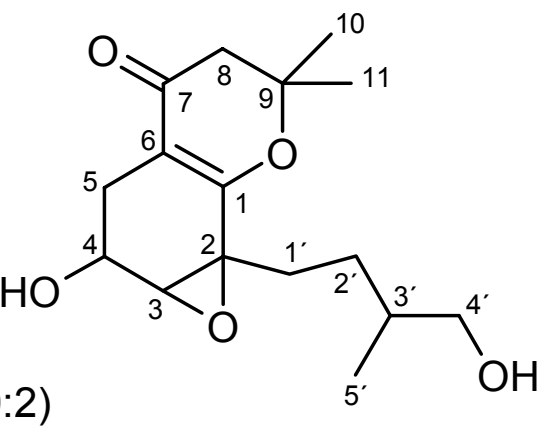
0.27 (Essigester/Methanol 19:1).

Anfärbeverhalten: pink (Anis), blau (Ehrlich), gelb-braun (Orcin). 
$[\alpha]_{D}^{20}=-74^{\circ}(\mathrm{c}=1.0 \mathrm{mg} / \mathrm{mL}$ in Methanol).

ESI-MS: $\mathrm{m} / \mathrm{z}=319.1[\mathrm{M}+\mathrm{Na}]^{+}, 615.1[2 \mathrm{M}+\mathrm{Na}]^{+}, 297.1[\mathrm{M}+\mathrm{H}]^{+}, 295.0[\mathrm{M}-\mathrm{H}]^{-}$.

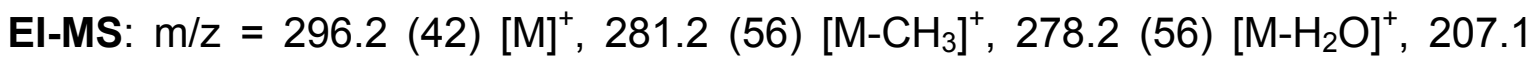
(16), 181.1 (24), 171.1 (16), 83.1 (100) $\quad\left[\mathrm{CO}-\mathrm{CH}=\mathrm{C}\left(\mathrm{CH}_{3}\right)_{2}\right]^{+}, \quad 55.1$ (61) $\left[\mathrm{CH}=\mathrm{C}\left(\mathrm{CH}_{3}\right)_{2}\right]^{+}$.

IR $(\mathrm{KBr}): \tilde{v}=3420,2930,2874,1649,1609,1410,1383,1326,1256,1168,1123$, $1091,1057,1035,919 \mathrm{~cm}^{-1}$.

UV $(\mathrm{MeOH}) \quad: \lambda_{\max }(\varepsilon)=220$ (2084), 286 (6470);

$(\mathrm{MeOH} / \mathrm{HCl}) \quad: \lambda_{\max }(\varepsilon)=286(6398)$;

$(\mathrm{MeOH} / \mathrm{NaOH}): \lambda_{\max }(\varepsilon)=286(6835) \mathrm{nm}$.

CD $(\mathrm{MeOH}): \lambda_{\max }[\Theta]=201$ (4583), 210 (-919), 220 (1420), 242 (-109), 319 $(-8152) \mathrm{nm}$.

${ }^{1} \mathrm{H}-\mathrm{NMR}\left(600 \mathrm{MHz}, \mathrm{D}_{5}\right.$-Pyridin, $35^{\circ} \mathrm{C}$ ): $\delta_{\mathrm{H}}=1.09$ (d, $\left.\mathrm{J}=6.5 \mathrm{~Hz}, 3 \mathrm{H}, 5^{\prime}-\mathrm{H}_{3}\right), 1.33$ (s, $\left.3 \mathrm{H}, 11-\mathrm{H}_{3}\right), 1.36\left(\mathrm{~s}, 3 \mathrm{H}, 10-\mathrm{H}_{3}\right), 1.58\left(\mathrm{~m}, 1 \mathrm{H}, 2^{\prime}-\mathrm{H}_{\mathrm{A}}\right), 1.77$ (ddd, $J=13.5,12.0$, $\left.5.0 \mathrm{~Hz}, 1 \mathrm{H}, 1^{\prime}-\mathrm{H}_{\mathrm{A}}\right), 1.88\left(\mathrm{~m}, 1 \mathrm{H}, 3^{\prime}-\mathrm{H}\right), 2.03$ (ddt, $J=13.0,11.5,5.5 \mathrm{~Hz}, 1 \mathrm{H}, 2^{\prime}-\mathrm{H}_{\mathrm{B}}$ ), 2.41 (ddd, $\left.J=13.5,11.5,4.5 \mathrm{~Hz}, 1 \mathrm{H}, 1^{\prime}-\mathrm{H}_{\mathrm{B}}\right), 2.45$ (d, $\left.J=16.5 \mathrm{~Hz}, 1 \mathrm{H}, 8-\mathrm{H}_{\mathrm{A}}\right), 2.47$ (dd, $\left.J=16.0,5.0 \mathrm{~Hz}, 1 \mathrm{H}, 5-\mathrm{H}_{\mathrm{A}}\right), 2.57\left(\mathrm{~d}, J=16.5 \mathrm{~Hz}, 1 \mathrm{H}, 8-\mathrm{H}_{\mathrm{B}}\right.$ ), 3.29 (dt, $J=16.0$, $\left.2.5 \mathrm{~Hz}, 1 \mathrm{H}, 5-\mathrm{H}_{\mathrm{B}}\right), 3.69\left(\mathrm{dd}, J=10.5,6.0 \mathrm{~Hz}, 1 \mathrm{H}, 4^{\prime}-\mathrm{H}_{\mathrm{A}}\right), 3.73(\mathrm{dd}, J=10.5$, $6.0 \mathrm{~Hz}, 1 \mathrm{H}, 4^{\prime}-\mathrm{H}_{\mathrm{B}}$ ), 3.78 (t, $\left.J=2.5 \mathrm{~Hz}, 1 \mathrm{H}, 3-\mathrm{H}\right), 4.72(\mathrm{dt}, J=5.0,2.5 \mathrm{~Hz}, 1 \mathrm{H}, 4-\mathrm{H})$, 5.88 (brs, $1 \mathrm{H}, \mathrm{OH}), 6.83$ (brs, $1 \mathrm{H}, \mathrm{OH}) \mathrm{ppm}$.

${ }^{13}$ C-NMR (150 MHz, D 5 -Pyridin, 35 $\mathrm{C}$ ): $\delta_{\mathrm{C}}=17.09$ (q, C-5'), 24.97 (q, C-10), 26.10 (t, C-5), 26.75 (q, C-11), 28.66 (t, C-1'), 29.63 (t, C-2'), 36.70 (d, C-3'), 47.36 (t, C-8), 57.93 (s, C-2), 63.32 (d, C-4), 63.83 (d, C-3), 67.48 (t, C-4'), 81.00 (s, C-9), 107.27 (s, C-6), 164.76 (s, C-1), 191.26 (s, C-7) ppm. 
3-Dimethylallyl-4-hydroxybenzoesäure $(\underline{96})$

$\mathrm{C}_{12} \mathrm{H}_{14} \mathrm{O}_{3}(206.24)$

$\mathbf{R}_{\mathbf{f}}$-Wert: 0.25 (Chloroform/Methanol 9:1)

0.52 (Cyclohexan/Essigester/Methanol 5:10:2)

0.51 (Essigester/Methanol 19:1).

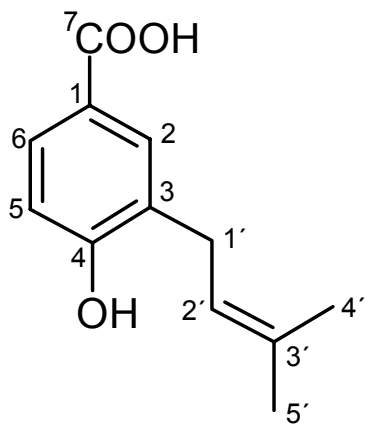

Anfärbeverhalten: leicht rosa (Ehrlich).

ESI-MS: $\mathrm{m} / \mathrm{z}=207.1[\mathrm{M}+\mathrm{H}]^{+}, 205.2[\mathrm{M}-\mathrm{H}]^{-}$.

El-MS: $m / z=206.1$ (63) $[\mathrm{M}]^{+}, 191.1$ (15) $\left[{\left.\mathrm{M}-\mathrm{CH}_{3}\right]}^{+}, 173.1\right.$ (8), 161.1 (12) [M$\mathrm{COOH}]^{+}, 151.1(100)\left[\mathrm{M}-\mathrm{CH}=\mathrm{C}\left(\mathrm{CH}_{3}\right)_{2}\right]^{+}$.

IR $(\mathrm{KBr}): \widetilde{v}=3395,2969,2926,1684,1603,1508,1437,1405,1380,1275,1172$, $1124,1091,924,834,775,630 \mathrm{~cm}^{-1}$.

UV $(\mathrm{MeOH}) \quad: \lambda_{\max }(\varepsilon)=203$ (18308), 255 (9159);

$(\mathrm{MeOH} / \mathrm{HCl}) \quad: \lambda_{\max }(\varepsilon)=202$ (11229), 217 (14560), 259 (10164);

$(\mathrm{MeOH} / \mathrm{NaOH}): \lambda_{\max }(\varepsilon)=205$ (28802), 251 (7104), 277 (6364) nm.

${ }^{1} \mathrm{H}-\mathrm{NMR}\left(600 \mathrm{MHz}, \mathrm{D}_{5}-\mathrm{Py}\right.$ ridin, $\left.35^{\circ} \mathrm{C}\right): \delta_{\mathrm{H}}=1.68\left(\mathrm{~s}, 3 \mathrm{H}, 5^{\prime}-\mathrm{H}_{3}\right), 1.73\left(\mathrm{~s}, 3 \mathrm{H}, 4^{\prime}-\mathrm{H}_{3}\right)$, $3.70\left(\mathrm{~d}, J=7.5 \mathrm{~Hz}, 2 \mathrm{H}, 1^{\prime}-\mathrm{H}_{2}\right), 5.64\left(\mathrm{tm}, J=7.5 \mathrm{~Hz}, 1.0,1 \mathrm{H}, 2^{\prime}-\mathrm{H}\right), 7.22$ (d, $J=8.5 \mathrm{~Hz}, 1 \mathrm{H}, 5-\mathrm{H}), 8.32(\mathrm{dd}, J=8.5,2.0 \mathrm{~Hz}, 1 \mathrm{H}, 6-\mathrm{H}), 8.48(\mathrm{~d}, J=2.0 \mathrm{~Hz}, 1 \mathrm{H}$, 2-H) ppm.

${ }^{13} \mathrm{C}-N M R\left(150 \mathrm{MHz}, \mathrm{D}_{5}-\mathrm{Pyridin}^{\circ} 35^{\circ} \mathrm{C}\right): \delta_{\mathrm{C}}=17.71$ (q, C-4'), 25.68 (q, C-5'), 28.97 (t, C-1'), 115.13 (d, C-5), 123.17 (d, C-2'), 128.85 (s, C-3), 129.97 (d, C-6), 132.27 (d, C-2), 132.38 (s, C-3'), 160.69 (s, C-4), 169.26 (s, C-7) ppm.

. ${ }^{1} \mathrm{H}-\mathrm{NMR}\left(600 \mathrm{MHz}, \mathrm{CD}_{3} \mathrm{OD}\right): \delta_{\mathrm{H}}=1.71\left(\mathrm{~s}, 3 \mathrm{H}, 5^{\prime}-\mathrm{H}_{3}\right), 1.74(\mathrm{~d}, J=1.0 \mathrm{~Hz}, 3 \mathrm{H}$, $4^{\prime}-\mathrm{H}_{3}$ ), 3.30 (brd, $J=7.5 \mathrm{~Hz}, 2 \mathrm{H}, 1^{\prime}-\mathrm{H}_{2}$ ), 5.31 (tm, $\left.J=7.5,1.5 \mathrm{~Hz}, 1 \mathrm{H}, 2^{\prime}-\mathrm{H}\right), 6.76$ (d, J = 8.5 Hz, 1H, 5-H), 7.69 (dd, J = 8.5, $2.0 \mathrm{~Hz}, 1 \mathrm{H}, 6-\mathrm{H}), 7.72(\mathrm{~d}, J=2.0 \mathrm{~Hz}$, $1 \mathrm{H}, 2-\mathrm{H}) \mathrm{ppm}$. 
${ }^{13}$ C-NMR (600 MHz, CD ${ }_{3} \mathrm{OD}$ ): $\delta_{\mathrm{C}}=17.82$ (q, C-4'), 25.97 (q, C-5'), 29.02 (t, C-1'), 115.20 (d, C-5), 123.17 (s, C-1), 123.32 (d, C-2'), 129.19 (s, C-3), 130.34 (d, C-6), 132.48 (d, C-2), 133.58 (s, C-3'), 160.81 (s, C-4), 171.00 (s, C-7) ppm.

4-Hydroxy-3-(3'-hydroxy-3'-methyl-but-1-enyl)-benzoesäure ( $\underline{97})$

$\mathrm{C}_{12} \mathrm{H}_{14} \mathrm{O}_{4}(222.24)$

$\mathbf{R}_{\mathbf{f}}$-Wert: 0.39 (Chloroform/Methanol 9:1)

0.49 (Cyclohexan/Essigester/Methanol 5:10:2)

0.48 (Essigester/Methanol 19:1).

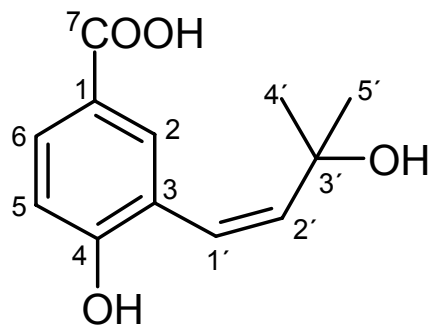

Anfärbeverhalten: hellblau (Ehrlich).

ESI-MS: $\mathrm{m} / \mathrm{z}=223.1[\mathrm{M}+\mathrm{H}]^{+}$.

IR (KBr): $\tilde{v}=3425,2973,2933,1676,1606,1446,1413,1368,1328,1299,1274$, $1200,1169,1123,1013,959,769 \mathrm{~cm}^{-1}$.

UV $(\mathrm{MeOH}) \quad: \lambda_{\max }(\varepsilon)=236$ (27754), 277 (3915);

$(\mathrm{MeOH} / \mathrm{HCl}) \quad: \lambda_{\max }(\varepsilon)=238$ (27699), 281 (4279);

$(\mathrm{MeOH} / \mathrm{NaOH}): \lambda_{\max }(\varepsilon)=202$ (22709), 234 (30027), 274 (4311) nm.

${ }^{1} \mathrm{H}-\mathrm{NMR}\left(600 \mathrm{MHz}, \mathrm{D}_{5}-\right.$ Pyridin, $\left.35^{\circ} \mathrm{C}\right): \delta_{\mathrm{H}}=1.39\left(\mathrm{~d}, \mathrm{~J}=1.0 \mathrm{~Hz}, 6 \mathrm{H}, 4^{\prime}-\mathrm{H}_{3}, 5^{\prime}-\mathrm{H}_{3}\right)$, $5.64\left(\mathrm{~d}, J=10.0 \mathrm{~Hz}, 1 \mathrm{H}, 1^{\prime}-\mathrm{H}\right.$ oder $\left.2^{\prime}-\mathrm{H}\right), 6.41\left(\mathrm{~d}, J=10.0 \mathrm{~Hz}, 1 \mathrm{H}, 1^{\prime}-\mathrm{H}\right.$ oder $\left.2^{\prime}-\mathrm{H}\right), 6.99(\mathrm{~d}, J=8.5 \mathrm{~Hz}, 1 \mathrm{H}, 5-\mathrm{H}), 8.14(\mathrm{~d}, J=2.5 \mathrm{~Hz}, 1 \mathrm{H}, 2-\mathrm{H}), 8.24$ (dd, $J=8.5$, $2.5 \mathrm{~Hz}, 1 \mathrm{H}, 6-\mathrm{H}) \mathrm{ppm}$.

${ }^{13} \mathrm{C}$-NMR $\left(150 \mathrm{MHz}, \mathrm{D}_{5}\right.$-Pyridin, $\left.35^{\circ} \mathrm{C}\right): \delta_{\mathrm{C}}=27.67\left(2 \times \mathrm{q}, \mathrm{C}^{\prime} 4^{\prime}, \mathrm{C}^{\prime} 5^{\prime}\right), 76.97(\mathrm{~s}$, C-3'), 115.89 (d, C-5), 120.60 (s, C-1), 121.47 (d, C-1'oder C-2'), 124.58 (s, C-3), 128.39 (d, C-1'oder C-2'), 130.86 (d, C-6), 131.23 (d, C-2), 156.64 (s, C-4), 168.13 (s, C-7) ppm. 


\subsection{Wachstumskurve}

Zur Aufnahme des zeitabhängigen Produktionsverlaufes von WDMH 46 wurde der Stamm in $1 \mathrm{~L}-$ Schüttelkolben ohne Schikanen, befüllt mit $250 \mathrm{~mL}$ Nährlösung 1405 , bei $28^{\circ} \mathrm{C}$ und $120 \mathrm{spm}$ kultiviert. Ab der 42 . Stunde wurden alle $12 \mathrm{~h}$ zwei Kolben entnommen und diese nach Standard (Abbildung 4) aufgearbeitet. Die erhaltenen Extrakte wurden dünnschichtchromatographisch untersucht.

\subsection{Biogeneseuntersuchungen}

\subsubsection{Fütterung von $\left[1-{ }^{13} \mathrm{C}\right] \mathrm{Acetat}$ und $\left[\mathrm{U}-{ }^{13} \mathrm{C}_{3}\right]$ Glycerin}

Die Fütterungsexperimente (1 L-Ansatz) wurden in jeweils vier $1 \mathrm{~L}-$ Kolben ohne Schikanen mit $250 \mathrm{~mL}$ Nährlösung 1405 durchgeführt. Nach dem Animpfen mit einem $3 \mathrm{~cm}^{2}$ großem Stück einer gut bewachsenen Agarplatte wurde der Stamm bei $28^{\circ} \mathrm{C}$ und $120 \mathrm{spm}$ inkubiert. Die zu fütternde Substanz wurde in bidestilliertem Wasser gelöst, die Lösung auf einen $\mathrm{pH}$-Wert von 6.5 eingestellt und autoklaviert.

\begin{tabular}{|c|c|c|c|c|}
\hline markierte Substanz & Firma & $\begin{array}{c}{[\%]} \\
\text { Anreicherung }\end{array}$ & {$[\mathrm{mg} / \mathrm{L}]$} & {$[\mathrm{mmol} / \mathrm{L}]$} \\
\hline Na-[1- $\left.{ }^{13} \mathrm{C}\right]$ Acetat & Campro Scientific & 99 & 750 & 9.1 \\
\hline$\left[\mathrm{U}-{ }^{13} \mathrm{C}_{3}\right]$ Glycerin & $\begin{array}{c}\text { Cambridge Isotope } \\
\text { Laboratories }\end{array}$ & 99 & 750 & 8.1 \\
\hline
\end{tabular}

Tabelle 17: Isotopenmarkierte Vorläufer für Biogeneseuntersuchungen der Lupallene.

Bei der Fütterung von markiertem Acetat wurden ab der 60./62. Stunde mittels einer Pumpe pro Kolben kontinuierlich $50 \mathrm{~mL}$ Lösung über 36 Stunden zugefüttert. Nach einer Kultivierungsdauer von 120/110 Stunden (24/12 Stunden Nachlauf) wurde der Ansatz geerntet und nach den allgemeinen Bedingungen aufgearbeitet. Im Experiment mit uniform markierten Glycerin wurde die Precursorlösung von der 53. bis 89. Stunde zugepumpt und der Fermentationsansatz nach der 96. Stunde geerntet. Als Referenz ließ man $1 \mathrm{~L}$ Kultur ohne Zufütterung mitlaufen. Das Myzel wurde verworfen und das Kulturfiltrat weiter bearbeitet. Der Kulturfiltrat-Extrakt wurde je nach produzierter Substanz an Kieselgel und Sephadex LH-20 säulenchromatographiert. 


\subsubsection{Fütterung potentieller Vorläufer}

$621.5 \mathrm{mg}(4.5 \mathrm{mmol} / \mathrm{L})$ p-Hydroxybenzoesäure, $630.5 \mathrm{mg}(4.5 \mathrm{mmol} / \mathrm{L})$ 2-Fluor-

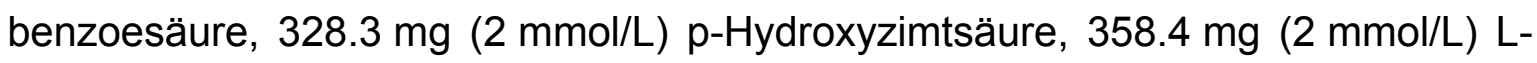
Phenylalanin und $362.4 \mathrm{mg} \mathrm{L}$-Tyrosin $(2 \mathrm{mmol} / \mathrm{L})$ wurden in $25 \mathrm{~mL}$ sterilem Wasser gelöst und mit $0.1 \mathrm{M} \mathrm{NaOH}$ und $0.05 \mathrm{M} \mathrm{HCl}$ auf $\mathrm{pH}=6.5$ eingestellt. Die je vier 1L-Kolben pro gefütterter Substanz ohne Schikane mit $250 \mathrm{~mL}$ Nährlösung 1405 wurden bei $28^{\circ} \mathrm{C}$ und $120 \mathrm{rpm}$ fermentiert. Je $1 \mathrm{~mL}$ der Precursorlösung pro Kolben wurden der Kultur ab der 60. Stunde sechsmal in Abständen von 12 Stunden durch einen Sterilfilter zugeführt. Die Kulturen wurden nach sechs Tagen geerntet und unter Standardbedingungen aufgearbeitet.

\subsection{Derivatisierungen}

\subsubsection{Acetylierung von Lupallen $\mathbf{C}(\underline{87})$}

$10.3 \mathrm{mg}(0.030 \mathrm{mmol}) \underline{87}$ wurden in $2 \mathrm{~mL}$ Dichlormethan gelöst und mit $1 \mathrm{~mL}$ Pyridin versetzt. Die Mischung wurde auf $0^{\circ} \mathrm{C}$ abgekühlt, anschließend $1 \mathrm{~mL}$ Acetanhydrid zugefügt und gerührt. Der Reaktionsverlauf wurde alle 10 min durch DC-Analyse des Reaktionsgemisches kontrolliert. Nach 2 Stunden beendete man die Reaktion durch Zugabe von 20 mL Eis-Wasser. Das Reaktionsgemisch wurde dreimal mit jeweils $20 \mathrm{~mL}$ Dichlormethan ausgeschüttelt, die organische Phase eingeengt und mit Toluol koevaporiert. Die dünnschichtchromatographische Analyse des Rohproduktes $(13.6 \mathrm{mg}$ ) zeigte den Spot einer Substanz. Nach Aufreinigung an Sephadex LH-20 (Aceton) konnte ein Acetylierungsprodukt gewonnen (98) werden. Ausbeute: $6.3 \mathrm{mg}(0.016 \mathrm{mmol}, 54 \%)$ farbloses Öl.

Lupallen C-4'-Acetat ( $\underline{98})$

$\mathrm{C}_{20} \mathrm{H}_{30} \mathrm{O}_{7}(382.45)$

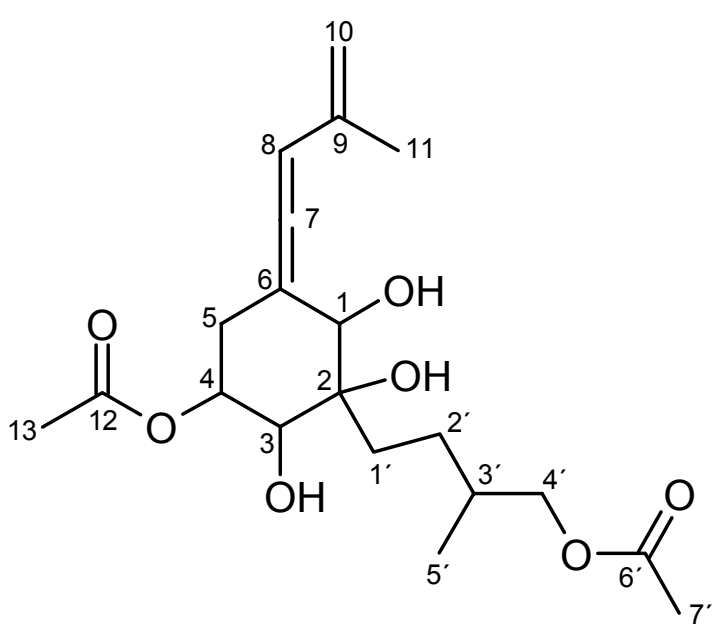


$\mathbf{R}_{\mathbf{f}}$-Wert: 0.48 (Chloroform/Methanol 9:1)

0.56 (Cyclohexan/Essigester/Methanol 5:10:2)

0.58 (Essigester/Methanol 19:1).

Anfärbeverhalten: pink (Anis), blau (Ehrlich), gelb-braun (Orcin).

DCI-MS: $\mathrm{m} / \mathrm{z}=400.2\left[\mathrm{M}+\mathrm{NH}_{4}\right]^{+}, 782.5\left[2 \mathrm{M}+\mathrm{NH}_{4}\right]^{+}$.

IR $(\mathrm{KBr}): \tilde{v}=3431,2964,2936,1955,1735,1719,1456,1435,1375,1245,1124$, $1035,977,886 \mathrm{~cm}^{-1}$.

UV $(\mathrm{MeOH}) \quad: \lambda_{\max }(\varepsilon)=220(18804)$;

$(\mathrm{MeOH} / \mathrm{HCl}) \quad: \lambda_{\max }(\varepsilon)=222(17696)$;

$(\mathrm{MeOH} / \mathrm{NaOH}): \lambda_{\max }(\varepsilon)=202$ (37748), 222 (20299) nm.

${ }^{1} \mathrm{H}-\mathrm{NMR}\left(600 \mathrm{MHz}, \mathrm{D}_{5}\right.$-Pyridin, $\left.35^{\circ} \mathrm{C}\right): \delta_{\mathrm{H}}=0.95\left(\mathrm{~d}, J=7.0 \mathrm{~Hz}, 3 \mathrm{H}, 5^{\prime}-\mathrm{H}_{3}\right), 1.74$ (dddd, $\left.J=12.5,12.5,8.0,4.5 \mathrm{~Hz}, 1 \mathrm{H}, 2^{\prime}-\mathrm{H}_{\mathrm{A}}\right), 1.86\left(\mathrm{~s}, 3 \mathrm{H}, 11-\mathrm{H}_{3}\right), 1.87(\mathrm{~m}, 1 \mathrm{H}$, $\left.3^{\prime}-\mathrm{H}\right), 1.94\left(\mathrm{~s}, 3 \mathrm{H}, 7^{\prime}-\mathrm{H}_{3}\right), 1.98\left(\mathrm{~s}, 3 \mathrm{H}, 13-\mathrm{H}_{3}\right), 2.03\left(\mathrm{~m}, 1 \mathrm{H}, 2^{\prime}-\mathrm{H}_{\mathrm{B}}\right), 2.36$ (ddd, $\left.J=13.5,12.5,4.5 \mathrm{~Hz}, 1 \mathrm{H}, 1^{\prime}-\mathrm{H}_{\mathrm{A}}\right), 2.45$ (ddd, $J=13.5,12.5,4.5 \mathrm{~Hz}, 1 \mathrm{H}, 1^{\prime}-\mathrm{H}_{\mathrm{B}}$ ), $2.76\left(\mathrm{dd}, J=12.5,5.0 \mathrm{~Hz}, 1 \mathrm{H}, 5-\mathrm{H}_{\mathrm{A}}\right), 3.40\left(\mathrm{td}, J=12.5,4.0 \mathrm{~Hz}, 1 \mathrm{H}, 5-\mathrm{H}_{\mathrm{B}}\right), 3.98$ (dd, $J=10.5,7.0 \mathrm{~Hz}, 1 \mathrm{H}, 4^{\prime}-\mathrm{H}_{\mathrm{A}}$ ), 4.11 (dd, $J=10.5,5.5 \mathrm{~Hz}, 1 \mathrm{H}, 4^{\prime}-\mathrm{H}_{\mathrm{B}}$ ), 4.48 (brs, $1 \mathrm{H}, 3-\mathrm{H}$ ), 4.56 (brd, $J=7.0 \mathrm{~Hz}, 1 \mathrm{H}, 1-\mathrm{H}$ ), 4.87 (quin, $J=1.0 \mathrm{~Hz}, 1 \mathrm{H}, 10-\mathrm{H}_{\mathrm{A}}$ ), 4.97 $\left(\mathrm{m}, 1 \mathrm{H}, 10-\mathrm{H}_{\mathrm{B}}\right), 6.00(\mathrm{ddd}, J=12.0,5.0,2.5 \mathrm{~Hz}, 1 \mathrm{H}, 4-\mathrm{H}), 6.06(\mathrm{~d}, J=4.0 \mathrm{~Hz}, 1 \mathrm{H}$, 8-H), 6.39 (brs, 1H, 2-OH), 6.45 (brd, J = 8.0 Hz, 1H, 1-OH), 7.03 (brd, J = $6.0 \mathrm{~Hz}$, $1 \mathrm{H}, 3-\mathrm{OH}) \mathrm{ppm}$.

${ }^{13}$ C-NMR (150 MHz, D $5^{-P y r i d i n, ~ 35 ㅇ) ~: ~} \delta_{\mathrm{C}}=17.18$ (q, C-5'), 19.85 (q, C-11), 20.71 (q, C-7'), 21.12 (q, C-13), 26.41 (t, C-2'), 26.64 (t, C-5), 33.66 (d, C-3'), 33.90 (t, C-1'), 69.58 (t, C-4'), 72.77 (d, C-4), 74.27 (d, C-3), 74.79 (s, C-2), 77.02 (d, C-1), 97.51 (d, C-8), 104.02 (s, C-6), 113.87 (t, C-10), 140.05 (s, C-9), 170.41 (s, C-6'), 170.78 (s, C-12), 204.52 (s, C-7) ppm. 


\subsection{2. ortho-Brombenzoat von Lupallen C (ㅎ7)}

$11.4 \mathrm{mg}$ (0.033 mmol) $\underline{87}$ wurden in $6 \mathrm{~mL}$ Dichlormethan gelöst und mit $8.4 \mathrm{mg}$ (1 eq.) 2-Brombenzoesäure, $12.1 \mathrm{mg}$ (1.5 eq.) EDCl und katalytischen Mengen an DMAP (3 mg) versetzt. Die Mischung wurde gerührt und der Reaktionsverlauf durch DC-Kontrolle verfolgt. Da nach zwei Stunden immer noch Edukt vorlag, fügte man erneut Säure (3 mg) und $\operatorname{EDCl}(3 . \mathrm{mg}$ ) hinzu und rührte weitere zwei Stunden. Anschließend gab man ca. $50 \mathrm{~mL}$ Wasser und noch ca. $20 \mathrm{~mL}$ Dichlormethan zur Reaktionsmischung, extrahierte gegeneinander und engte die organische Phase im Vakuum ein. Die dünnschichtchromatographische Analyse des Rohproduktes zeigte eine ca. 60 \%ige Umsetzung. Nach Reinigung an Kieselgel $\left(\mathrm{CH}_{2} \mathrm{Cl}_{2} / \mathrm{MeOH}\right.$ 19:1) konnte neben dem Benzoylierungsprodukt $\underline{99}$ Edukt zurück gewonnen werden. Eine abschließende Säulenchromatographie an Sephadex $\mathrm{LH}-20\left(\mathrm{CH}_{2} \mathrm{Cl}_{2} / \mathrm{MeOH} 1: 1\right)$ lieferte $5.9 \mathrm{mg}$ Brombenzoat mit einer Ausbeute von $34 \%$.

\section{Lupallen C-4'-o-brombenzoat (모)}

$\mathrm{C}_{25} \mathrm{H}_{31} \mathrm{O}_{7} \mathrm{Br}(523.42)$

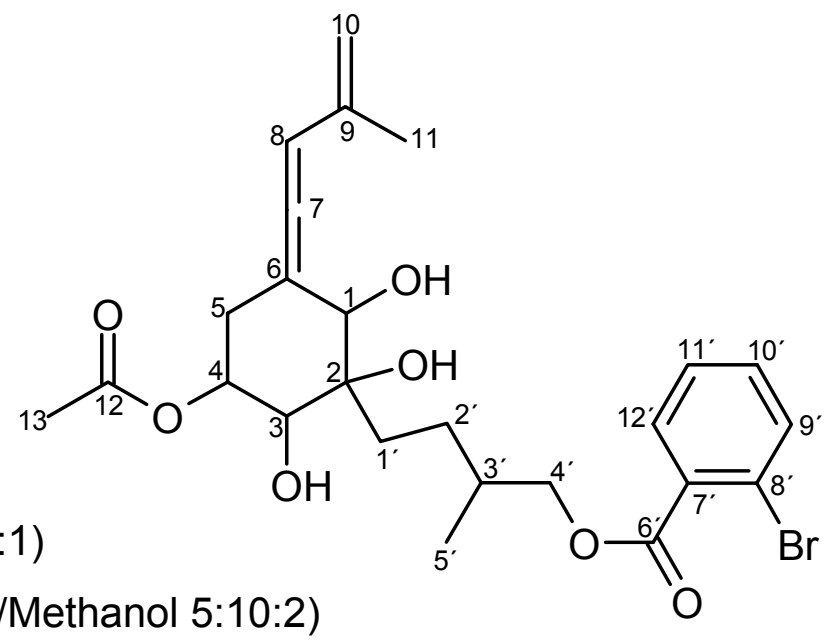

0.62 (Cyclohexan/Essigester/Methanol 5:10:2)

0.63 (Essigester/Methanol 19:1).

Anfärbeverhalten: pink (Anis), grün-violett (Ehrlich), beige-braun (Orcin).

ESI-MS: $\mathrm{m} / \mathrm{z}=545.2[\mathrm{M}+\mathrm{Na}]^{+}, 1068.7[2 \mathrm{M}+\mathrm{Na}]^{+}$.

IR $(\mathrm{KBr}): \tilde{v}=3425,2964,2929,1955,1719,1459,1434,1376,1293,1251,1134$, 1116, 1030, $972,748 \mathrm{~cm}^{-1}$.

UV $(\mathrm{MeOH}) \quad: \lambda_{\max }(\varepsilon)=202$ (31080), 222 sh (18901), 283 (1745);

$(\mathrm{MeOH} / \mathrm{HCl}) \quad: \lambda_{\max }(\varepsilon)=201$ (22908), 221 (19774), 283 (2036); 
$(\mathrm{MeOH} / \mathrm{NaOH}): \lambda_{\max }(\varepsilon)=204$ (46349), $223 \mathrm{sh}$ (20937), 281 (291) nm.

${ }^{1} \mathrm{H}-\mathrm{NMR}\left(600 \mathrm{MHz}, \mathrm{D}_{5}\right.$-Pyridin, $\left.35^{\circ} \mathrm{C}\right): \delta_{\mathrm{H}}=1.07\left(\mathrm{~d}, J=6.5 \mathrm{~Hz}, 3 \mathrm{H}, 5^{\prime}-\mathrm{H}_{3}\right), 1.84(\mathrm{~m}$, $\left.1 \mathrm{H}, 3^{\prime}-\mathrm{H}_{\mathrm{A}}\right), 1.87\left(\mathrm{~s}, 3 \mathrm{H}, 11-\mathrm{H}_{3}\right), 1.87\left(\mathrm{~m}, 1 \mathrm{H}, 3^{\prime}-\mathrm{H}\right), 1.98\left(\mathrm{~s}, 3 \mathrm{H}, 13-\mathrm{H}_{3}\right), 2.04(\mathrm{~m}$, $1 \mathrm{H}, 2^{\prime}-\mathrm{H}_{\mathrm{A}}$ ), $2.14\left(\mathrm{tt}, J=12.5,5.5 \mathrm{~Hz}, 1 \mathrm{H}, 2^{\prime}-\mathrm{H}_{\mathrm{B}}\right), 2.47\left(\mathrm{~m}, 2 \mathrm{H}, 1^{\prime}-\mathrm{H}_{2}\right), 2.78$ (dd, $\left.J=12.5,5.0 \mathrm{~Hz}, 1 \mathrm{H}, 5-\mathrm{H}_{\mathrm{A}}\right), 3.42\left(\mathrm{td}, J=12.5,4.0 \mathrm{~Hz}, 1 \mathrm{H}, 5-\mathrm{H}_{\mathrm{B}}\right), 4.27$ (dd, $\left.J=10.5,6.5 \mathrm{~Hz}, 1 \mathrm{H}, 4^{\prime}-\mathrm{H}_{\mathrm{A}}\right), 4.42\left(\mathrm{dd}, J=10.5,5.5 \mathrm{~Hz}, 1 \mathrm{H}, 4^{\prime}-\mathrm{H}_{\mathrm{B}}\right.$ ), 4.51 (brs, $1 \mathrm{H}$, 3-H), 4.59 (brs, $1 \mathrm{H}, 1-\mathrm{H}$ ), 4.87 (quin, $J=1.0 \mathrm{~Hz}, 1 \mathrm{H}, 10-\mathrm{H}_{\mathrm{A}}$ ), $4.97\left(\mathrm{~m}, 1 \mathrm{H}, 10-\mathrm{H}_{\mathrm{B}}\right.$ ), 6.01 (ddd, $J=11.5,5.0,2.5 \mathrm{~Hz}, 1 \mathrm{H}, 4-\mathrm{H}), 6.06$ (d, $J=4.0 \mathrm{~Hz}, 1 \mathrm{H}, 8-\mathrm{H}), 6.44$ (brs, $1 \mathrm{H}, 2-\mathrm{OH}$ ), 6.47 (brd, $J=6.0 \mathrm{~Hz}, 1 \mathrm{H}, 1-\mathrm{OH}), 7.07(\mathrm{brd}, J=3.0 \mathrm{~Hz}, 1 \mathrm{H}, 3-\mathrm{OH}) 7.24$ (td, $J=7.5,2.0 \mathrm{~Hz}, 1 \mathrm{H}, 10^{\prime}-\mathrm{H}$ ), 7.28 (td, $J=7.5,1.5 \mathrm{~Hz}, 1 \mathrm{H}, 11^{\prime}-\mathrm{H}$ ), 7.65 (dd, $\left.J=7.5,1.5 \mathrm{~Hz}, 1 \mathrm{H}, 9^{\prime}-\mathrm{H}\right), 7.88\left(\mathrm{dd}, J=7.5,2.0 \mathrm{~Hz}, 1 \mathrm{H}, 12^{\prime}-\mathrm{H}\right) \mathrm{ppm}$.

${ }^{13} \mathrm{C}-$ NMR (150 MHz, D 5 -Pyridin, 35ㅇ): $\delta_{\mathrm{C}}=17.38$ (q, C-5'), 19.87 (q, C-11), 21.12 (q, C-13), 26.48 (t, C-2'), 26.65 (t, C-5), 33.79 (d, C-3'), 33.94 (t, C-1'), 70.88 (t, C-4'), 72.78 (d, C-4), 74.33 (d, C-3), 74.82 (s, C-2), 76.99 (d, C-1), 97.52 (d, C-8), 104.00 (s, C-6), 113.87 (t, C-10), 121.56 (s, C-8'), 127.72 (d, C-12'), 131.57 (d, C-9'), 132.82 (d, C-11'), 133.55 (s, C-7'), 134.50 (d, C-10'), 140.05 (s, C-9), 166.47 (s, C-6'), 170.41 (s, C-12), 204.53 (s, C-7) ppm.

\subsection{3. para-Brombenzoat von Lupallen A ( $\underline{\mathbf{8 6}})$}

Eine Lösung von $50.3 \mathrm{mg}(0.180 \mathrm{mmol}) \underline{\mathbf{8}}$ in $8 \mathrm{~mL}$ Dichlormethan wurde mit $54.2 \mathrm{mg}$ (1.5 eq.) 4-Brombenzoesäure, $51.6 \mathrm{mg}$ (1.5 eq.) EDCl und katalytischen Mengen an DMAP (4 mg) versetzt. Die Mischung wurde gerührt und der Reaktionsverlauf durch DC-Kontrolle verfolgt. Mit der Umsetzung des Eduktes entstanden vier bis fünf Reaktionsprodukte. Die Reaktion wurde nach fünf Stunden durch Zugabe von $40 \mathrm{~mL}$ Wasser abgebrochen. Nach dem Zusatz von $35 \mathrm{~mL}$ Dichlormethan wurde die organische Phase zweimal mit Wasser extrahiert und im Vakuum eingeengt. Der erhaltene schwach gelbliche, viskose Rückstand (116 mg) wurde an Kieselgel $\left(\mathrm{CH}_{2} \mathrm{Cl}_{2} / \mathrm{MeOH}\right.$ 19:1) säulenchromatographiert und ergab sechs Fraktionen. Die polarste der Fraktionen (6) beinhaltete $3.2 \mathrm{mg}$ Edukt. Fraktion 1 (16.4 mg) und 2 (13.6 mg) wurden vereinigt und einem erneuten Reinigungsschritt an Sephadex LH-20 (Dichlormethan) unterzogen. Man erhielt 
$13.6 \mathrm{mg}(0.021 \mathrm{mmol}, 12 \%)$ nahezu reines Lupallen A-4,4'-di-para-brombenzoat (100), das im Kolben breiig auskristallisierte.

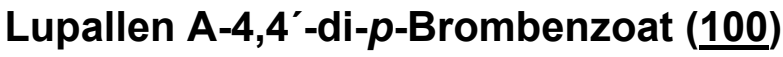

$\mathrm{C}_{30} \mathrm{H}_{30} \mathrm{O}_{6} \mathrm{Br}_{2}(646.37)$

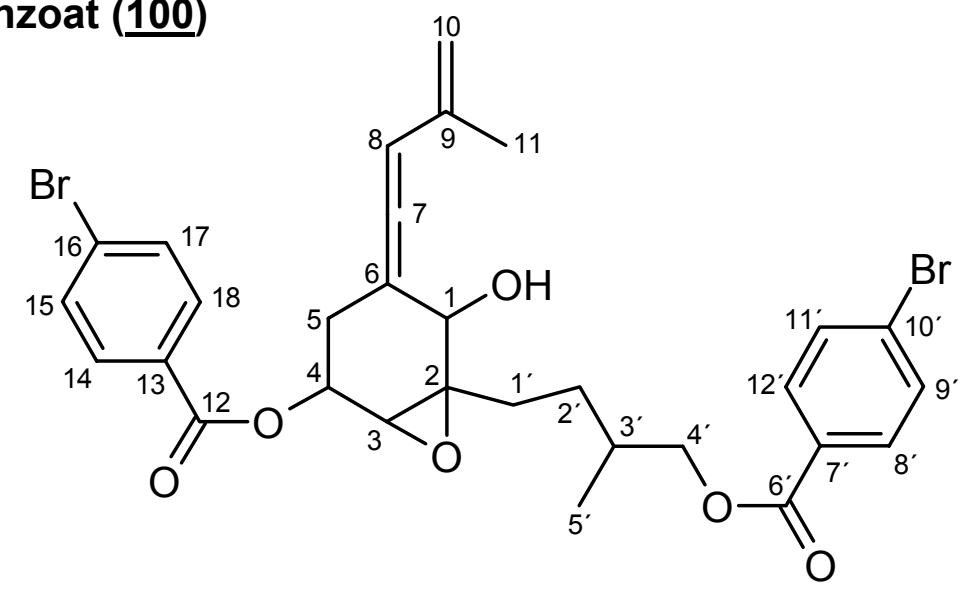

$\mathbf{R}_{\mathbf{f}}$-Wert: 0.67 (Chloroform/Methanol 49:1)

0.70 (Cyclohexan/Essigester/Methanol 5:10:2)

0.65 (Essigester/Methanol 19:1).

Anfärbeverhalten: pink (Anis), blau-grau (Ehrlich), grün-braun (Orcin).

El-MS: $\mathrm{m} / \mathrm{z}=646.1(1)[\mathrm{M}]^{+}, 431.1(5), 184.0(100)\left[\mathrm{Br}-\mathrm{C}_{6} \mathrm{H}_{4}-\mathrm{CO}\right]^{+}, 175.2(35), 85$ (48).

IR (KBr): $\tilde{v}=3462,2960,2925,1956,1720,1590,1398,1270,1173,1102,1096$, $1012,847,756,683 \mathrm{~cm}^{-1}$.

UV $(\mathrm{MeOH}) \quad: \lambda_{\max }(\varepsilon)=204$ (55581), 223 sh (34878), 242 (50634);

$(\mathrm{MeOH} / \mathrm{HCl}) \quad: \lambda_{\max }(\varepsilon)=204$ (77804), 224 sh (34238), 243 (49806);

$(\mathrm{MeOH} / \mathrm{NaOH}): \lambda_{\max }(\varepsilon)=204$ (77804), $242(51748) \mathrm{nm}$.

${ }^{1} \mathrm{H}-\mathrm{NMR}\left(600 \mathrm{MHz}, \mathrm{D}_{5}\right.$-Pyridin, $\left.35^{\circ} \mathrm{C}\right): \delta_{\mathrm{H}}=0.98\left(\mathrm{~d}, J=6.5 \mathrm{~Hz}, 3 \mathrm{H}, 5^{\prime}-\mathrm{H}_{3}\right), 1.61(\mathrm{~m}$, $\left.1 \mathrm{H}, 2^{\prime}-\mathrm{H}_{\mathrm{A}}\right), 1.75\left(\mathrm{~s}, 3 \mathrm{H}, 11-\mathrm{H}_{3}\right), 1.86-1.93\left(\mathrm{~m}, 2 \mathrm{H}, 1^{\prime}-\mathrm{H}_{\mathrm{A}}, 3^{\prime}-\mathrm{H}\right) 1.96\left(\mathrm{~m}, 1 \mathrm{H}, 2^{\prime}-\mathrm{H}_{\mathrm{B}}\right)$, $2.53\left(\mathrm{~m}, 1 \mathrm{H}, 1^{\prime}-\mathrm{H}_{\mathrm{B}}\right), 2.87\left(\mathrm{dd}, J=13.0,7.0 \mathrm{~Hz}, 1 \mathrm{H}, 5-\mathrm{H}_{\mathrm{A}}\right), 3.04$ (ddd, $J=13.0,9.0$, $\left.3.0 \mathrm{~Hz}, 1 \mathrm{H}, 5-\mathrm{H}_{\mathrm{B}}\right), 3.58(1 \mathrm{H}, 3-\mathrm{H}), 4.22\left(\mathrm{dd}, \mathrm{J}=11.0,6.5 \mathrm{~Hz}, 1 \mathrm{H}, 4^{\prime}-\mathrm{H}_{\mathrm{A}}\right), 4.29$ (dd, $\left.J=11.0,5.5 \mathrm{~Hz}, 1 \mathrm{H}, 4^{\prime}-\mathrm{H}_{\mathrm{B}}\right), 4.82\left(\mathrm{brs}, 1 \mathrm{H}, 10-\mathrm{H}_{\mathrm{A}}\right), 4.86\left(\mathrm{~m}, 1 \mathrm{H}, 10-\mathrm{H}_{\mathrm{B}}\right), 5.01$ (brs, $1 \mathrm{H}, 1-\mathrm{H}), 5.67(\mathrm{dd}, J=8.5,7.0 \mathrm{~Hz}, 1 \mathrm{H}, 4-\mathrm{H}), 6.16(\mathrm{~d}, J=3.0 \mathrm{~Hz}, 1 \mathrm{H}, 8-\mathrm{H})$, 7.56-7.60 (m, 2H, 14-H, 18-H), 7.64-7.67 (m, 2H, 8'-H, 12'-H), 7.98-8.02 (m, 4H, $\left.15-\mathrm{H}, 17-\mathrm{H}, 9^{\prime}-\mathrm{H}, 12^{\prime}-\mathrm{H}\right) \mathrm{ppm}$. 
${ }^{13}$ C-NMR (150 MHz, D 5 -Pyridin, 35ㅇ): $\delta_{\mathrm{C}}=17.12$ (q, C-5'), 19.73 (q, C-11), 27.25 (t, C-2'), 28.14 (t, C-5), 29.95 (t, C-1'), 33.19 (d, C-3'), 60.22 (d, C-3), 64.09 (s, C-2), 69.30 (d, C-1), 69.63 (d, C-4), 69.86 (t, C-4'), 98.82 (d, C-8), 101.61 (s, C-6), 114.43 (t, C-10), 128.07 (s, C-16), 128.55 (s, C-10'), 129.49 (s, C-7'), 129.99 (s, C-13), 131.52 (2 x d, C-14, C-18), 131.77 (2 x d, C-8', C-12'), 132.11 (2 x d, C-9', C-11'), 132.16 (2 x d, C-15, C-17), 139.57 (s, C-9), 165.18 (s, C-7'), 165.78 (s, C-12), 204.03 (s, C-7) ppm. 


\section{LITERATURVERZEICHNIS}

1. a.) W. TANG, G. EISENBRAND, Chinese Drugs of Plant Orign, Sprinter-Verlag, Berlin, 1992.

b.) X. LOZOYA in Spektrum der Wissenschaft Spezial 6, 1998, 10.

2. J. Falbe, M. Regitz, RÖMPP Lexikon Chemie, Band 2: Cm-G, Thieme Verlag, Stuttgart-New York, 1997, 1203.

3. S. EBEL, H.J. Roth, Lexikon der Pharmazie, Thieme Verlag, Stuttgart-New York, 1987, 488.

4. a.) H.W. Florey, E. Chain, N.G. Heatley, M.A. Jennings, A.G. Sanders, E.P. Abraham, M.E. Florey, Antibiotics, Oxford University Press, Oxford, 1949.

b.) WIKIPEDIA, Die freie Enzyklopädie, unter www.wikipedia.org/wiki/penizillin.

5. P. PORTIER in Spektrum der Wissenschaft Spezial 6, 1997, 6.

6. V. BetINA, Mycotoxins: Chemical, biological and environmental aspects in Bioactive Molecules Vol. 9, Elsevier, Amsterdam-Oxford-New York-Tokyo, 1989, 243.

7. Alsberg, Black, U.S.D.A., Bur. Plant. Industry, Bulletin. 1913, 270.

8. A. Fleming, Brit. J. Pat. 1929, 10, 226.

9. E.P. Abraham, W. Baker, E. Chain, Nature 1942, 356.

10. E.P. Abraham, P.B. Loder, Cephalosporin $C$ in Cephalosporins and Penicillins: Chemistry and Biology, (Hrsg.: E.H. FLINN), Academic Press, New York, 1972.

11. G.G.F. NeWton, E.P. Abraham, Nature 1955, 175, 548.

12. U. GRÄFE, Biochemie der Antibiotoka: Struktur-BiosyntheseWirkmechanismus, Spektrum Akademischer Verlag GmbH, Heidelberg, 1992.

13. E.K. SCHMITT, U. KUCK, BIOspektrum 2002, 1, 22.

14. Autorekollektiv, Extremiphile: Mikroorganismen in ausgefallenen Lebensräumen, (Hrsg.: K. HAusmanN, B.P. KREMER), VCH-Verlag, Weinheim, 1994. 
15. a.) G. BLunden, Phytother. Res. 2001, 15, 89.

b.) R. Ulber, M. Arndt, T. Hanke, M. Laroche, K. Ackermann, T. Scheper, BIOforum 1999, 12, 773.

16. D.J. FaulkneR, Nat. Prod. Rep. 2000, 17, 1.

17. a.) D.J. FAulkner, Nat. Prod. Rep. 2000, 17, 7.

b.) D.J. Faulkner, Nat. Prod. Rep. 2001, 18, 1.

c.) D.J. FAulkner, Nat. Prod. Rep. 2002, 19, 1.

d.) J.W. Blunt, B.R. Copp, M.H.G. Munro, P.T. Northcote, M.R. Prinsep, Nat. Prod. Rep. 2003, 20, 1.

e.) J.W. Blunt, B.R. Copp, M.H.G. Munro, P.T. Northcote, M.R. Prinsep, Nat. Prod. Rep. 2004, 21, 1.

18. A. Numata, C. Takahashi, J. Ito, T. Takada, K. Kawal, Y. Usami,

E. Matsumura, M. Imachi, T. Ito, T. Hasegawa, Tertrahedron Lett. 1993, 34, 2355.

19. a.) A. Numata, C. Takahashi, J. Ito, K. Minoura, T. Yamada, T. Matsuda, K. Numoto, J. Chem. Soc., Perkin Trans. 1 1996, 239.

b.) C. Inamoto, T. Yamada, Y. Ito, K. Minoura, A. Numata, Tetrahedron 2001, 57, 2355.

20. C. Takahashi, A. Numata, T. Yamada, K. Minoura, S. Enomoto, K. Konishi, M. NAKAI, C. MATSUDA, K. NomOtO, Tetrahedron Lett. 1996, 37, 655.

21. C. Inamoto, K. Minoura, S. Hagashita, K. Nomoto, A. Numata, J. Chem. Soc., Perkin Trans. 1 1998, 449.

22. C. Inamoto, K. Minoura, T. OKa, T. Otha, S. hagashita, A. numata, Tetrahedron 1999, 55, 14353.

23. T.S. Bugni, C.M. IRELANd, Nat. Prod. Rep. 2004, 21, 143.

24. L.A. McDonald, D.R. Abbanat, L.R. Barbieri, V.L. Bernan, C.M. Discafani, M. Greenstein, K. JonatA, J.D. Korshalla, P. Lassota, M. Tischler, G.T. CARTER, Tetrahedron Lett. 1992, 40, 2489.

25. W. FenicAL, Chem. Rev. 1993, 93, 1673.

26. Y. LIN, X. WU, S. FENG, G. JIANG, S. ZHOU, L.L.P. VRIJMOED, E.B.G. JONES, Tetrahedron Lett. 2001, 42, 449.

27. J. Kohlmeyer, E. Kohlmeyer, Marine Mycology: The higher Fungi, Academic Press, London 1979. 
28. a.) P.R. JENSEN, W. FENICAL, Secondary metabolites from marine fungi in Fungi in Marine Environment, (Hrsg.:K.D. HYDE), Fungal Diversity Press, Hong Kong, 2002, 293.

b.) U. Höller, A.D. Wright, G.F. Matthee, G.M. König, S. Dräger, H.J. Aust, B. Schulz, Mycol. Res. 2000, 104, 1354.

29. E.M. LEANO, Ecology of stramimipiles from mangrove habitats in Fungi in Marine Environment, (Hrsg.:K.D. HYDE), Fungal Diversity Press, Hong Kong, 2002.

30. R. Siepmann, W. KöHNK, Über Hefen und einige Pilze aus dem Nordatlantik in Veröffentlichungen des Institutes für Meeresforschung Bremerhaven 1962, 8, 79.

31. J. Kohlmeyer, B. Volkmann-Kohlmeyer, Canadian Journal of Botany 1990 , 68, 1554.

32. D.B. Strongman, J.D. Miller, L. Calhoun, J.A. Findley, N.J. Whitney, Botanica Marina 1987, 30, 21.

33. J. Sonnenbichler, J. Dietrich, P. PeipP, Biol Chem. Hoppe-Seyler 1994, $375,71$.

34. P.R. Jensen, W. Fenical in Drugs from the Sea, (Hrsg.: N. Fusetani), Karger, Basel, 2000, 6.

35. M. Cueto, P.R. Jensen, C. Kaufman, W. Fenical, E. Lobkovsky, J. Clardy, J. Nat. Prod. 2001, 64, 1444.

36. R. HöFs, M. WALKeR, Angew. Chem. 2000, 109, 3400.

37. S.A. Wachsman, H.A. LeCheVAlier, Science 1949, 109, 305.

38. a.) U. Edlund, A. Hensel, D. Fröse, U. Pfüller, A. Scheffler in Die Mistel in der Tumortherapie - Grundlagenforschung und Klinik, (Hrsg.: R. SCHEER, R. BAUER, P.A. Berg, V. FintelmanN), Wechselwirkungen zwischen Beerenpolysacchariden und Lektin der weißbeerigen Mistel, KVC Verlag, Essen, 2001, 64.

b.) G.M. Stein, U. Edlund, G. Schaller, U. Pfüller, A. Büsing, M. SCHIETZEL in Die Mistel in der Tumortherapie - Grundlagenforschung und Klinik, (Hrsg.: R. Scheer, R. BAuer, P.A. Berg, V. FintelmanN), 
Polysaccharide und Viscotoxine - Immunologische Wirkungen und Interaktionen, KVC Verlag, Essen, 2001, 145.

39. S. OMURA, The search for bioactive microbial compounds from microorganism, Springer Verlag, Berlin-Heidelberg-New York, 1992.

40. R.P. Hertzberg, A.J. Pope, Curr. Opin. Chem. Biol. 2000, 4, 445.

41. S. Umezawa, T. Tsuchiya, K. Tatsuna, Y. HoRIYchi, T. Usi, H. UmezaWa, M. HAMAdA, A. YAGI, J. Antibiot. 1970, 40, 953.

42. H. ZäHner, H. Drautz, W. Weber in Bioactive Microbial Product-Search and Discovery (Hrsg.: J.D. BULLOCK, L.J. NISBET, D.J. WINSTLEY), Academic Press, London-New York, 1982, 51.

43. S. Grabley, R. Thiericke, A. Zeeck in The Chemical Search Approach in Drug Discovery, (Hrsg.: S. GRABLEY, R. THIERICKE), Springer Verlag, BerlinHeidelberg-New York, 1999, 124.

44. H.P. FiedLeR, J. Nat. Prod. 1993, 2, 119.

45. a.) W. IHN, S. HEINZE, U. GRÄFE, BIOspektrum 1997, 3, 39.

b.) M. Stadler, T. Henkel, H. Mueller, K. Weber, H. Schlecker, J. Chromatogr. 1998, 818, 187.

46. G. Brinkmann, K. Messer, M. Wohlfahrt, J. Kraus, K. Dumbuya, M. RÜCKERT, Anal. Chem. 1999, 71, 2678.

47. H. LAATSCH, Naturstoffdatenbank Antibase, Version 2002, Chemical Concepts, Weinheim 2000.

48. J. Wink, S. Grabley, M. Gareis, A. Zeeck, S. Philipps, Eur. Pat. Appl. 1993, EP 456475 A1 19930616.

49. P. Bloch, C. Tamm, P. Bollinger, T.J. Petcher, H.P. Weber, Helv. Chem. Acta 1976, 59, 133.

50. P. Bloch, C. TAmm, Helv. Chem. Acta 1981, 64, 304.

51. W. Breitenstein, K.K. CheXal, P. Mohr, C. Tamm, Helv. Chem. Acta 1981, 64, 379.

52. D. Komagata, S. Fujita, N. Yamashita, S. Saito, T. Morino, J. of Antibiot. 1996, 49, 958.

53. D. KomagatA, T. HaYAOKA, Jpn. Kokai, Tokkyo Koho 1995, JP 07258263.

54. J. Wenke, H. ANKe, O. SteRneR, Biosci. Biotechnol. Biochem. 1993, 57, 961. 
55. R.J. Cole, R.H. Cox, Handbook of Toxic Fungal Metabolites, 1981, Academic Press INC.

56. S. Ohmomo, T. Sato, T. Utagawa, M. Abe, Agr. Biol. Chem. 1975, 39, 1333.

57. KEGUCHI et al., Heterocycles 1982, 19, 1873.

58. B. Kopp-Holtwiesche, H.J. Rehm, J. Environ. Pathol. Toxicol. Oncol. 1990, $10,41$.

59. P.M. Scott, M.A. Merrien, J. Polonsky, Experimentia 1976, 32, 140.

60. R.J. Cole, J.W. KiRKSeY, J.W. DORner, D.M. Wilson, J.C. JOHnSON, A.N. Johnson, D.M. Bedell, J.P. Springer, K.K. CheXal, J.C. Clardy, R.H. CoX, J. Agric. Food Chem. 1977, 25, 826.

61. J.S. SPILSBury, S. Wilkinson, J. Chem. Soc. 1961, 2085.

62. B. Arnoux, M.A. Merrien, C. Pascard, J. Polonsky, P. Scott, J. Chem. Research 1978, S, 210.

63. N.J. BaCh, H.E. BoAz, E.C. Kornfeld, C.J. Chang, H.G. Floss, E.W. Hagaman, E. Wenkert, J. Org. Chem. 1974, 39, 1272.

64. C.-B. CUI, H. KaKeyA, G. OKADA, J. of Antibiot. 1996, 49, 534.

65. M. Kondoh, T. Usul, T. Mayumi, H. OsadA, J. of Antibiot. 1998, 51, 801.

66. Petra Alvermann, Dissertation: „Untersuchungen zur Biosynthese und Stereochemie von Hormaomycin sowie Strukturaufklärung ausgewählter Metabolite des Pilzes Aspergillus fumigatus“, Cuvillier Verlag, Göttingen, 2001.

67. S. NozOE, M. GoI, N. MoRISAKI, Tetrahedron Lett. 1970, 15, 1293.

68. B.E. Gross, R.E. MARKWELL, J.C. SteWART, Tetrahedron 1971, 27, 1663.

69. J.R. HANson, P.B. HitChCOCK, R. NyFElER, J. Chem. Soc., Trans / 1975, 1586.

70. R. Evans, J.R. Hanson, R. NyFeler, J. Chem. Soc., Trans I, 1976, 1214.

71. H.G. Cutler, J.M. Jacyno, R.S. Phillips, R.L. von Tersch, D. Cole, N. MontemurRo, Agric. Biol. Chem. 1991, 55, 243.

72. D.E. Cane, R. Iyengar, M. S. Shiao, J. Am. Chem. Soc. 1981, 103, 914.

73. K. Umehara, K. Yoshida, M. OKamoto, M. IWami, H. Tanaka, M. Kohsaka, H. IMANAKA, J. Antibiot. 1984, 37, 469. 
74. J.H. Birkinshaw, J.C. Roberts, P. Roffey, J. Chem. Soc. 1966, C, 855.

75. A. Craig et AlL, Tetrahedron Lett. 1986, 27, 887.

76. AutORENKOLlektiv, SciFinder Scholar, American Chemical Society, 2001.

77. Autorenkollektiv, Chapman \& Hall / CRC Dictionary of Natural Products on CD-ROM, Version 12:1, Chapman \& Hall, CRC, 2003.

78. P.J. Augengamp, C.W. HolzApfel, Journal of the South African Chemical Institute 1970, 23, 40.

79. R.P. Maskey, I. GRÜn-Wollny, H. LaAtsch, J. of Antibiot. 2003, 56, 459.

80. D.G.I. Kingston, P.N. Chen, J.R. Vercellotti, Phytochemistry 1976, 15, 1037.

81. R.F. Keller, A.T. Tu, Handbook of Natural Toxins Vol.1: Plant and Fungal Toxins, Marcel Dekker INC., New York, 1983.

82. W.P. BLounT, Turkeys (J. Br.Turkey Fed.) 1961, 9, 52/55/61/77.

83. B.F. Nesbitt, J. O’Kelly, K. Sargean, Nature (London) 1962, 195, 1062.

84. A. Clegler, Lloydia, 1975, 38, 21.

85. J.P. Thaxton, H.T. Tung, P.B. Hamilton, Poult. Sci. 1965, 7083.

86. M. Nukina, S. Marumo, Agric. Biol. Chem. 1977, 41, 717.

87. W.A. Ayer, P.-P. Lu, H. Orszanska, J. Nat. Prod. 1993, 56, 1835.

88. J.A. Findley, D. KWAN, Can. J. Chem. 1973, 51, 3299.

89. T. MoritA, H. AOKI, Agric. Biol. Chem. 1974, 38, 1501.

90. S.K. Talapatra, D. Karmacharya, S.C. De, B. Talapatra, Phytochemistry, 1988, 27, 3929.

91. J.E. Knapp, P.-D. Chau, P.L. SchifF, JR. \& D.J. Slatkin, J. Chem. Res. 1979, $S, 236$.

92. A. Fukami, T. Nakamura, Y.-P. Kim, K. Shiomi, M. Hayashi, T. Nagal, H. Yamada, K. Komiyama, S. OmuRa, J. Antibiot. 2000, 53, 1215.

93. P.-D. Chau, P.L. Schiff, D.J. Slatkin, J.E. Knapp, M. ChaU, R.D. Rosenstein, Llyodia. 1975, 38, 213.

94. M. Chau, P.L. Schiff, D.J. Slatkin, J.E. Knapp, Acta Cryst, Sect.B, 1975, 31, 2937.

95. M. Bös, C. Rolf, N. Inoue-Ohga, A. Nakano, Y. Takehana, A.J. Sleight, Bioorg. Med. Chem. 1997, 5, 2165. 
96. H. Nakajima, S. Nakamura, H. Fujimoto, K. Fukuyama, T. Hamasaki, J. Nat. Prod. 1997, 60, 414.

97. H. NAKAJIMA, Developments in Plant Pathology, 1997, 13 (Molecular Genetics of Host-Specific Toxins in Plant Disease), 71.

98. T. Murakami, Y. Morikawa, M. Hashimoto, T. Okuno, Y. Harada, Organic Letters 2004, 6, 157.

99. T.J. Simpson, D.J. Stenzel, J. Chem .Soc., Chem. Comm. 1981, 239.

100. T.J. Simpson, D.J. Stenzel, J. Chem. Soc., Chem. Comm. 1982, 1074.

101. B.J. Auret, D.R. Boyd, P.M. Robinson, C.G. Watson, J. Chem .Soc., Chem. Comm. 1971, 24, 1585.

102. R. Shan, M. Stadler, H. Anke, O. Sterner, J. Nat. Prod. 1997, 60, 804.

103. а.) A. PFIFFNER, Dissertation: Isolierung und Konstitutionsermittlung von Marticin und Isomarticin, zwei neue Welketoxinen aus Fusarium Martii, (E.Hardegger), ETH Zürich, 1963.

b.) H. KeRn, S. NAef-Roth, Phytopathol. Z. 1965, 53, 45.

C.) H. Kern, S. Naef-Roth, H. Item, Phytopathol. Z. 1970, 67, 1.

104. a.) P.W. Brian, H.G. Hemming, J.S. Muffatt, C.H. Unwin, Trans. Br. Mycol. Soc. 1953, 36, 243.

b.) A.J. Birch, L. LOH, A. Pelter, J.H. Birkinshow, P. Chaplen, A.H. Manchanda, M. Riano-Martin, Tetrahedron Lett. 1965, 29.

c.) A.J. BiRCh, J.H. BiRkinshow, P. Chaplen, L. Mo, A.H. ManchandA, M. Riano-Martin, Aust. J. Chem. 1969, 22, 1933.

105. J.E. Holenstein, H. Kern, A. Stoessl, J.B. Stothers, Tetrahedron Lett., 1983, 24, 4059.

106. A.J. Birch, F. Gager, A. Pelter, J.J. Wright, Aust. J. Chem. 1969, 22, 2429.

107. C.N. LeWis, J. Staunton, D.C. Sunter, J. Chem. Soc. Perkin Trans. I, 1988, 747.

108. a.) S. PATAI, The Chemistry of Ketenes, Allenes and Related Compounds, Wiley, New York, 1980.

b.) S.R. LANDOR, The Chemistry of the Allenes, Academic Press, London, 1982.

c.) H.F. Schuster, G.M. CoppolA, Allenes in Organic Synthesis, Wiley, New York, 1984. 
d.) C.J. ELSEVIER in Methods of Organic Chemistry (Houben-Weyl), Vol. E21a (Hrsg.: G. Helmchen, R.W. Hoffmann, J. Mulzer, E. SchaumanN), Thieme, Stuttgart, 1995, 537.

e.) N. KRAuSE, A.S.K. Hashmi, Modern Allene Chemistry, Wiley-VCH, Weinheim, 2004.

109. J.H. Van't HofF, La Chimie dans L'Espace, Bazendijk, Rotterdam, 1875.

110. A. Hoffmann-Röder, N. Krause, Angew. Chem., 2004, 116, 1216.

111. a.) S.R. LANDOR, "Naturally Occurring Allenes" in The Chemistry of the Allenes (Hrsg.: S. R. Landor), Academic Press, London, 1982, 679.

b.) A. ClAESSON, "Biologically Active Allenes" in The Chemistry of the Allenes (Hrsg.: S. R. Landor), Academic Press, London, 1982, 709.

c.) C.H. RoBinson, D.F. CoveY, "Biological Formation and Reactions" in The Chemistry of Ketenes, Allenes and Related Compounds (Hrsg.: S. Patai), Wiley, Chichester, 1980, 451.

d.) Eine im August 2003 durchgeführte Recherche in der Beilstein-Datenbank BS0302PR (Allen mit maximalen Freiheitsgraden im Struktureditor, inp $=^{*}$ im Fakteditor) ergab 194 Treffer. Nach Berücksichtigung von Mehrfachnennungen, Fehleinträgen sowie einiger in dieser Datenbank nicht enthaltener Verbindungen verblieben 146 allenische Naturstoffe (38 lineare Allene, 46 allenische Carotinoide und Terpenoide, 33 Bromallene, 29 weitere Allene) und 4 Naturstoffe mit Cumulen-Struktur.

112. a.) E.A. Johnson, K.L. BuRdON, J. Bacteriol. 1947, 54, 281.

b.) W.D. Celmer, I.A. Solomons, J. Am. Chem. Soc. 1952, 74, 1870; 2245, 3838.

113. D.F. HoRler, J. Chem. Soc. 1970, (C), 859.

114. a.) P.J. Kocienski, G. Cernigliaro, G. Feldstein, J. Org. Chem. 1977, 42, 353.

b.) R.W. Lang, E. Kohl-Mines, H.-H. Hansen, Helv. Chim. Acta 1985, 68, 2249.

C.) N. Krause, A. Gerold, Angew. Chem. 1997, 109, 194.

115. a.) W.H. PIRKLE, C.W. Boeder, J. Org. Chem. 1978, 43, 2091.

b.) K. MoRI, T. NukAdA, T. EBATA, Tetrahedron 1981, 37, 1343.

116. T. Satoh, N. Hanaki, Y. Kuramochi, Y. InOUE, K. Hosoya, K. Sakal, Tetrahedron 2002, 58, 2533. 
117. M. Franck-Neumann, D. Martina, D. NefF, Tetrahedron: Asymmetry 1998, 9, 697.

118. R. Willstätter, H.J. Page, Justus Liebigs Ann. Chem. 1914, 404, 237.

119. R. Bonnett, A.A. Spark, J.L. Tee, B.C.L. Weedon, Proc. Chem. Soc. London $1964,419$.

120. a.) J. Meinwald, K. Erickson, M. Hartshorn, Y.C. MeinWAld, T. Eisner, Tetrahedron Lett. 1968, 2959.

b.) T.E. DeVille, M.B. Hursthouse, S.W. Russell, B.C.L. Weedon, J. Chem. Soc. Chem. Commun. 1969, 754.

121. a.) A. Jensen, Acta Chem. Scand. 1964, 18, 2005.

b.) R. Bonnett, A.K. Mallams, J.L. Tee, B.C.L. Weedon, A. McCormick, J. Chem. Soc. Chem. Commun. 1966, 515.

c.) R. Bonnett, A.K. Mallams, A.A. Spark, J.L. Tee, B.C.L. Weedon,

A. McCormick, J. Chem. Soc. 1969, (C), 429.

d.) T.E. DeVille, M.B. Hursthouse, S.W. Russell, B.C.L. Weedon, J. Chem. Soc. Chem. Commun. 1969, 1311.

122. Y. Shiraga, K. Okano, T. Akira, C. Fukaya, K. Yokoyama, S. Tanaka, H. FUKUI, M. TABATA, Tetrahedron 1988, 44, 4703.

123. T. Rezanka, V.M. Dembitsky, Phytochemistry 2001, 56, 869.

124. R. KInNEL, A.J. Duggan, T. EISNER, J. MeInWALD, Tetrahedron Lett. 1977, 3913.

125. a.) A. FuKUZAWA, E. KuRosaWA, Tetrahedron Lett. 1979, 2797.

b.) M. SuzUKI, K. Kolzumi, H. KIKUCHI, T. SuzUKI, E. Kurosawa, Bull. Chem. Soc. Jpn. 1983, 56, 715.

C.) A.D. Wright, G.M. Koenig, O. Sticher, J. Nat. Prod. 1991, 54, 1025.

d.) M. Suzuki, Y. Sasage, M. IkURa, K. Hikichi, E. Kurosawa, Phytochemistry 1989, 28, 2145.

126. a.) N. Fusetani, M. Asano, S. Matsunaga, K. Hashimoto, Tetrahedron Lett. 1987, 28, 5837.

b.) S. Hokama, J. Tanaka, T. Higa, N. Fusetani, M. Asano, S. Matsunaga,

K. HASHIMOTO, Chem. Lett. 1988, 855.

C.) N. Fusetani, M. Asano, S. Matsunaga, K. Hashimoto, Tetrahedron 1989, $45,1647$. 
d.) M. Ochi, K. Kataoka, A. Tatsukawa, H. Kotsuki, K. Shibata, Heterocycles 1994, 38, 151.

e.) J.-R. Rho, H.-S. Lee, Y. Seo, K.W. Cho, J. Shin, J. Nat. Prod. 2000, 63, 254.

127. H.J. KwON, J.H. KIM, H.J. JUNG, Y.-G. KWON, M.Y. KIM, J.-R. RHO, J. SHIN, J. Microbiol. Biotechnol. 2001, 11, 656.

128. a.) T. Tokuyama, K. Uenoyama, G. Brown, J.W. Daly, B. Witkop, Helv. Chim. Acta 1974, 57, 2597.

b.) C.E. Spivak, M.A. Maleque, A.C. Oliveira, L.M. Masukawa, T. Tokuyama, J.W. Daly, E.X. Albuquerque, Mol. Pharmacol. 1982, 21, 351.

c.) T. Tokuyama, J. Yamamoto, J.W. Daly, R.J. Highet, Tetrahedron 1983, $39,49$.

d.) T. Tokuyama, N. Nishimori, I.L. Karle, M.W. EdWARds, J.W. Daly, Tetrahedron 1986, 42, 3453.

129. J.W. Daly, B. Witkop, T. Tokuyama, T. Nishikawa, I.L. Karle, Helv. Chim. Acta 1977, 60, 1128.

130. T.F. Spande, P. Jain, H.M. Garaffo, L.K. Pannell, H.J.C. Yeh, J.W. Daly, S. FuKumoto, K. Imamura, T. TOKUYAMA, J.A. TORRES, R.R. SNelLING, T.H. JONES, J. Nat. Prod. 1999, 62, 5.

131. C. WALSH, Tetrahedron 1982, 38, 871.

132. J.-M- Renaud, G. Tsoupras, H. Stoeckli-Evans, R. Tabacchi, Hev. Chem. Acta 1989, 72, 1262.

133. D.R. Sanson, H. Gracz, M.S. Tempesta, D.S. Fukuda, W.M. NakatSUKasa, T.H. SANDS, P.J. BAKER, J.S. MYNDERSE, Tetrahedron 1991, 47, 3633.

134. H.J. SCHIEWE, Dissertation (ISBN 3-932325-30-3), Universität Göttingen 1997.

135. R. HöFS, Dissertation: "Anwendung der OSMAC-Methode zur Gewinnung neuer Naturstoffe und Biosyntheseuntersuchungen an den Gabosinbildnern Streptomyces cellulosae S1096 und S1013" (ISBN 3-932325-74-5), Universität Göttingen 1999.

136. C. PUDER, Dissertation (ISBN 3-932325-30-3), Universität Göttingen 1997.

137. W. CRUeger, A. CRUeger, Lehrbuch der angewandten Mikrobiologie, Oldenbourg Verlag, München, 1984.

138. G.J. CLARK, D. LANGLEY, M.E. BuSHeLL, Microbiology 1995, 141, 663. 
139. ACD-Labs 6.0, Advanced Chemistry Development, INC., 1997-2004.

140. P. Venkatasubbaiah, W.S. Chilton, J. Nat. Prod. 1991, 54, 1293.

141. R. Bonnett, A.K. mallams, A.A. spark, J.L. tee, B.C.L. Weedon,

A. McCormick, J. Chem. Soc. 1969, C, 429.

142. T. BJÖRNLAND, Phytochemistry 1982, 21, 1715.

143. S. Isoe, S.B. Hyeon, H. Ichikawa, S. Katsumura, T. Sakan, Tetrahedron Letters 1968, 5561.

144. M. Mousse von CANet, J.-P. DALle, J.-C. MANI, Tetrahedron Letters 1968, 6037.

145. C.S. FoOte, M: BRENNER, Tetrahedron Letters 1968, 6041.

146. T.E. DeVille, J. Mora, M.B. Hursthouse, T.P. Toube, B.C.L. Weedon, Chem. Comm. 1970, 1231.

147. S. Isoe, S. Katsumura, S.B. Hyeon, T. Sakan, Tetrahedron Lett. 1971, 1089.

148. K. Bernhard, G.P. Moss, Gy. Toth, B.C.L. Weedon, Tetrahedron Lett. 1974, 44, 3899.

149. I.A. SWIFT, B.V. MILBORROW, Biochem. J. 1981, 199, 69.

150. J.M. Renaud, G. Tsoupras, R. Tabacchi, Helv. Chim. Acta 1989, 72, 929.

151. W. KNÖss, Pharmazie in unserer Zeit 1999, 28, 247.

152. S.L. Spurgeon, G.W. PORTER, in Biosynthesis of Isoprenoid Compounds, (Hrsg.: J.W. Porter, S.L. SpuRgeON, John WILEY and Sons), Vol. 1, New York, 1981, 1.

153. N. QURESHI, G.W. PORTER, in Biosynthesis of Isoprenoid Compounds, (Hrsg.: J.W. Porter, S.L. Spurgeon, John Wiley and Sons), Vol. 1, New York, $1981,47$.

154. M. Rohmer, Nat. Prod. Rep. 1999, 16, 565.

155. F. LÜTKe-Brinkhaus, H. Kleinig, Planta 1987, 171, 401.

156. D.J. McGarvey, R. Croteau, Plant Cell 1995, 7, 1015.

157. M. Rohmer, M. KNANI, H. SAHM, Biochem. J. 1993, 295, 517.

158. G. Flesh, M. Rohmer, Eur. J. Biochem. 1988, 1755, 405.

159. M. Rohmer, M. Seemann, H. Sahm, J. Am. Chem. Soc. 1996, 118, 2564.

160. W. Eisenreivh, D. Arigoni, M. ZenK, Chemistry \& Biology 1998, 5, R221.

161. T. Kuzuyama, H. Seto, Nat. Prod. Rep. 2003, 20, 171.

162. M. RoHmER, A Mevalonate-independent Route to Isopentenyl Diphosphate in Comprehensive Natural Products Chemistry, (Hrsg.: S.D. BARTON, 
K. NaKAnIShI, O. Meth-Cohn), Elsevier Science Ltd., Amsterdam-Lausanne, 1999.

163. H. BrockmanN, Justus Liebigs Ann. Chem. 1935, 521, 1.

164. V.P. Papageorgiou, A.N. Assimopoulou, E.A. Couladouros, D. Hepworth, K.C. Nicolaou, Angew. Chem. 1999, 111, 280.

165. V.P. PAPAgEORgIOU, Experimentia 1978, 34, 1499.

166. M. TABATA, Shokubutsu Soshiki Baiyo 1996, 13, 117.

167. J. Mann, Chemical Aspects of Biosynthesis, Oxford Chemistry Primers, 20, (Hrsg.: S.G. Davies), Oxford University Press, Oxford, 1994,31.

168. R. Löschler, L. Heide, Plant Physiol. 1994, 106, 271.

169. a.) K. YaZAKI, L. Heide, M. Tabata, Phytochemistry 1991, 30, 2233.

b.) J.-P. Schnitzler, J. Madlung, A. Rose, H.U. Seitz, Planta 1992, 188, 594.

C.) C.J. French, C.P. Vance, G.H.N. Towers, Phytochemistry 1976, 15, 564.

170. a.) H.V. SCHMIDT, M.H. ZENK, Tetrahedron Letters 1971, 4151.

b.) H. Inouye, S. Ueda, K. Inoue, H. Katsumura, Phytochemistry 1979, 18, 1301.

c.) T. Окамото, K. Yatzaki, M. TAвata, Phytochemistry 1995, 38, 83.

171. C. HERTWECK, B.S. MOORE, Tetrahedron 2000, 56, 9115.

172. MERCK, Anfärbereagenzien für Dünnschicht- und Papierchromatographie, E. MERCK, Darmstadt, 1998.

173. H.G. ScHLEGEL, Allgemeine Mikrobiologie, Georg Thieme Verlag, Stuttgart, 1992. 


\section{$\underline{\text { DANKE }}$}

Ein großes Dankeschön richtet sich an Hans-Peter Kroll und Michaela Klingebiel, die mich mit chromatographischen Trennungen und dem Messen experimenteller Daten unterstützt haben.

Für die Durchführung von Fermentationen im großen Maßstab danke ich HansJörg Langer und Jutta Gerber-Nolte, wie auch für die Bereitstellung von Testplatten.

Allen Mitgliedern der NMR-Abteilung und den Abteilungsoperatoren danke ich für die gute Zusammenarbeit. Besonders Herrn Reinhard Machinek sei für die Bereitstellung von reichlich Messzeit und seine stete Diskussionsbereitschaft gedankt. Für die Einarbeitung und gute Zusammenarbeit am 500er möchte ich Philipp Krastel danken sowie Carola Zolke, die mit Tipps und Tricks häufig weiterhelfen konnte. Ein Dankeschön auch an Christiane Siebert für so manches Messzeit tauschen.

Für die Aufnahme von Massenspektren gilt mein Dank Herrn Holm Frauendorf und seinen Mitarbeiterinnen Frau Györgyi Udvarnoki und Frau Gabriela Krökel. An dieser Stelle sage ich Danke zu Karsten Ströch und Luise Hoffmann für das Messen der HPLC-MS-Proben.

Den Computerbeauftragten der Abteilung, Jens Bitzer und Gerald Thormann, sei auf diesem Wege gedankt für die Betreuung, Wartung und Reparatur der Rechner und für die Hilfe bei Computerfragen.

Mein besonderer Dank geht an Andrea Schulze und Luise Hoffmann für das sorgfältige und engagierte Korrekturlesen dieses Skriptes.

Gerald Thormann danke ich für das stetig wechselnde Arbeitsklima in Z5 :). Danke auch an Sven Meyer, der mir die Zeit im Schreibraum versüßt hat und an Luise Hoffmann, die sich als würdige Nachfolgerin erwiesen hat.

Bei allen Mitgliedern meiner Arbeitsgruppe bedanke ich mich für die freundschaftliche Atmosphäre, die Hilfsbereitschaft und die vielen schönen Stunden im Kaffeeraum.

Nicht zuletzt ein großes DANKE an meine Eltern, meine Schwester und meine Freunde.

Um Andy meinen Dank auszusprechen, reichen Worte nicht. 


\section{Lebenslauf}

Ich wurde am 25.03.1976 als Tochter von Rita Wolff, geb. Kohs, und Gerhard Otto Wolff in Schwedt/Oder geboren.

Von September 1982 bis Juli 1991 besuchte ich die Polytechnische Oberschule Gustav Bruhn in Angermünde. Ab September 1991 war ich Schülerin des EinsteinGymnasiums in Angermünde, welches ich im Juni 1995 mit der Allgemeinen Hochschulreife verließ.

Zum Wintersemester 1995/96 begann ich mit dem Studium der Chemie an der Georg-August-Universität zu Göttingen und legte im Oktober 1997 die DiplomChemiker-Vorprüfung ab. In der Zeit von Januar bis September 2000 fertigte ich im Arbeitskreis von Prof. Dr. A. Zeeck meine Diplomarbeit mit dem Titel „Beiträge zur Biosynthese von Asperloxin A und Strukturaufklärung von Aspercyclinon" an und absolvierte im November 2000 die Diplom-Chemiker-Hauptprüfung.

Seit Januar 2001 arbeite ich unter Anleitung von Prof. Dr. A. Zeeck an der vorliegenden Dissertation. 